JULIO ROMEL MARTINEZ BOLAÑOS

ANÁLISE DA ESTABILIDADE DINÂMICA EM MINIRREDES COM CONTROLE AUTÔNOMO DE FREQUÊNCIA E TENSÃO

Tese apresentada à Escola

Politécnica da Universidade de São

Paulo para obtenção do título de

Doutor em Ciências

São Paulo 
JULIO ROMEL MARTINEZ BOLAÑOS

\section{ANÁLISE DA ESTABILIDADE DINÂMICA EM MINIRREDES COM CONTROLE AUTÔNOMO DE FREQUÊNCIA E TENSÃO}

Tese apresentada à Escola

Politécnica da Universidade de São

Paulo para obtenção do título de

Doutor em Ciências

Área de Concentração:

Sistemas de Potência

Orientadora:

Profa. Dra. Eliane Aparecida

Faria Amaral Fadigas

São Paulo 
Este exemplar foi revisado e alterado em relação à versão original, sob responsabilidade única do autor e com a anuência de seu orientador.

São Paulo, 15 de outubro de 2012.

Assinatura do autor

Assinatura do orientador

FICHA CATALOGRÁFICA

Martinez Bolaños, Julio Romel

Análise da estabilidade dinâmica de minirredes com controle autônomo de frequência e tensão / J.R. Martinez Bolaños. -ed.rev. -- São Paulo, 2012.

220 p.

Tese (Doutorado) - Escola Politécnica da Universidade de São Paulo. Departamento de Engenharia de Energia e Automação Elétricas.

1. Sistemas elétricos de potência 2. Sistemas dinâmicos 3. Geração de energia elétrica 4. Estabilidade de sistemas I. Universidade de São Paulo. Escola Politécnica. Departamento de Engenharia de Energia e Automação Elétricas II. t. 


\section{DEDICATÓRIA}

- Em especial aos meus queridos pais: Pablo Martínez e Mariana Bolaños, pelo incentivo, compreensão, carinho e amor. Suas palavras de motivação foram fundamentais nesta jornada.

- Aos meus queridos irmãos: Pablo Aníbal, Maria Elena e Cesar Antonio, assim como a Raquel Elizabeth e Blanca Narvaez que sempre acreditaram em mim.

- Às minhas tias Teresa Bolaños e Laura Bolaños (in memoriam). Infelizmente elas não puderam acompanhar esta grande conquista.

- Aos meus tios Vicente, Miguel, Rosa Amada e Cecilia pelo carinho e motivação.

- À Maria Benedita, pelo apoio constante nos momentos difíceis desta jornada. 


\section{AGRADECIMENTOS}

Gratidão em primeiro lugar a Deus por ter me mantido com vida e saúde, capacitando-me nos momentos de desafio e concedendo paciência sempre que meu limite era atingido.

À minha orientadora, Professora Doutora Eliane Aparecida Faria Amaral Fadigas, pela paciência, estímulo e sábia orientação ao longo destes quatro anos, sem a qual não teria sido possível a realização deste trabalho. E acima de tudo, por ser uma grande amiga, especialmente nos momentos mais difíceis.

Aos colegas do GEPEA-NER, Núcleo de Energias Renováveis do Departamento de Energia e Automação Elétricas da Universidade de São Paulo, que auxiliaram na realização deste trabalho.

À Coordenação de Aperfeiçoamento de Pessoal de Nível Superior (CAPES) pelo apoio financeiro disponibilizado para a realização desta tese de doutorado.

À todos os professores do Departamento de Energia e Automação Elétricas da USP que, de uma maneira ou outra, contribuíram para a realização e o sucesso deste trabalho.

A todos vocês, os meus mais sinceros agradecimentos. 


\section{RESUMO}

Cargas e fontes elétricas de pequeno porte, integradas através de alimentadores de distribuição de baixa tensão, agrupadas e gerenciadas de tal maneira que se comportem como uma única entidade controlável dentro de um sistema de potência e com a capacidade de operar de forma paralela ou isolada da rede elétrica convencional, constituem-se em um novo tipo de sistema elétrico conhecido como Minirrede.

Esta tese aborda o problema relacionado à estabilidade dinâmica de Minirredes com controle autônomo de frequência e tensão, quando operadas de forma isolada da rede convencional. As minirredes analisadas são compostas por combinações de fontes elétricas convencionais e fontes alternativas que não geram naturalmente em 50 ou $60 \mathrm{~Hz}$, sendo necessária a utilização de inversores para sua conexão com a rede elétrica.

A análise se realiza no espaço de estados com metodologias baseadas nas teorias que envolvem os autovalores e autovetores da matriz de estado. Para isto, modelos no espaço de estados são desenvolvidos para cada componente da Minirrede, mantendo um compromisso entre precisão e complexidade.

O caráter analítico da análise permite investigar a relação entre a estabilidade da Minirrede e os parâmetros do sistema, tais como ganhos dos controladores, dimensionamento da rede, configurações de geração, entre outros. Análises complementares de sensibilidade dos autovalores aos parâmetros elétricos do sistema permitem inferir o comportamento dinâmico de diversas configurações de Minirredes, obtendo-se importantes conclusões à respeito.

Os resultados analíticos são verificados através de simulação computacional no ambiente Simulink/Matlab ${ }^{\circledR}$.

Palavras-chave: Minirrede, estabilidade dinâmica, inversores. 


\begin{abstract}
Electrical loads and small power sources, integrated through low voltage distribution feeders, managed in such a way to behave as one controllable entity within in a power system, and with the ability to operate connected or non-connected to the conventional power system is a new kind of electrical system known as Microgrid.

This thesis addresses the problem related to dynamic stability of island Microgrids with autonomous control of frequency and voltage. The Microgrids analyzed are composed of combinations of conventional and alternative power sources that do not generate electricity naturally in 50 or $60 \mathrm{~Hz}$, therefore inverters are needed to provide AC network interface.

The analysis is performed in the state space with methodologies based on theories involving the eigenvalues and eigenvectors of the state matrix. For this, state-space models are developed for each component of Microgrid, maintaining a compromise between accuracy and complexity.

The analytical nature of this study allows investigating the relationship between the stability of Microgrid and parameters of the system, such as controller gains, network design, generation architectures, among others. Complementary sensitivity analyzes of eigenvalues to the electrical parameters of the system allow us to infer the dynamic behavior of various configurations of Microgrids, obtaining important conclusions on the matter.
\end{abstract}

The analytical results are verified by computer simulation using the platform Simulink/Matlab ${ }^{\circledR}$.

Keywords: Microgrids, dynamic stability, inverters 


\section{LISTA DE ILUSTRAÇÕES}

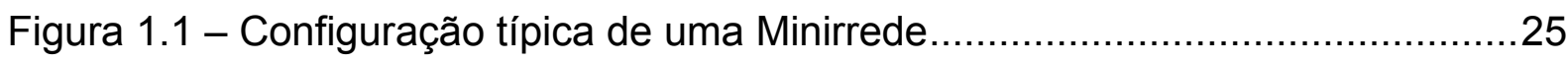

Figura 2.1 - Classificação da estabilidade em Sistemas de Potência........................37

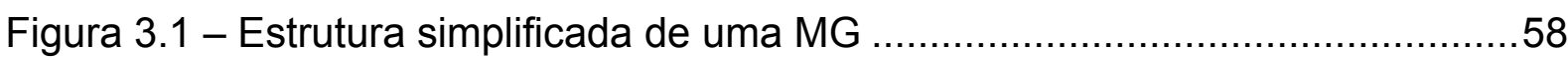

Figura 3.2 - Modelo completo no espaço de estados de uma MG ..........................60

Figura 3.3 - Circuitos equivalentes do estator e rotor de um gerador síncrono ........61

Figura 3.4 - Transformação de coordenadas d-q para D-Q....................................69

Figura 3.5 - Sistema de excitação estático IEEE - Tipo ST6B ………………......71

Figura 3.6 - Sistema de excitação estático ST6B - Simplificado ..............................73

Figura 3.7 - Diagrama de blocos reduzido - Sistema ST6B simplificado ..................73

Figura 3.8 - Diagrama de blocos do motor diesel com regulador ...........................77

Figura 3.9 - Esquema Básico de conexão dos Inversores .....................................83

Figura 3.10 - Diagrama de blocos de um Inversor com Controle $P$-Q $Q \ldots \ldots \ldots \ldots \ldots \ldots \ldots . . .84$

Figura 3.11 - Diagrama de blocos do PLL+Calculador de Potência-Controle P-Q ...85

Figura 3.12 - Diagrama de Blocos do Controlador de Potência - Controle P-Q.......87

Figura 3.13 - Diagrama de Blocos do Controlador de Corrente- Controle P-Q ........89

Figura 3.14 - Característica de estatismo (a) potência-ativa/frequência (b) potência-

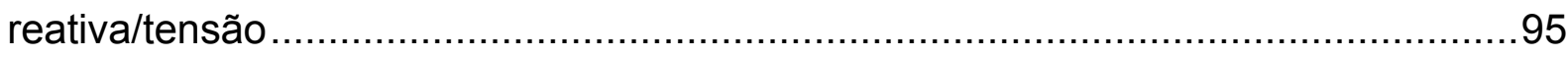

Figura 3.15 - Diagrama de blocos de um Inversor com Controle Droop..................96

Figura 3.16 - Diagrama de blocos do Calculador de Potência - Controle Droop .....97

Figura 3.17 - Diagrama de blocos do Gerenciador de Potência - Controle Droop...98

Figura 3.18 - Diagrama de blocos do Controlador de Tensão - Controle Droop......99

Figura 3.19 - Diagrama de blocos do Controlador de Corrente - Controle Droop..100

Figura 3.20 - Diagrama de blocos do Gerenciador de Potência - Controle Droop+F

Figura 3.21 - Circuitos equivalentes do estator e rotor de um motor de indução....112

Figura 3.22 - Conexão de Cargas de Impedância Constante R-L .........................115

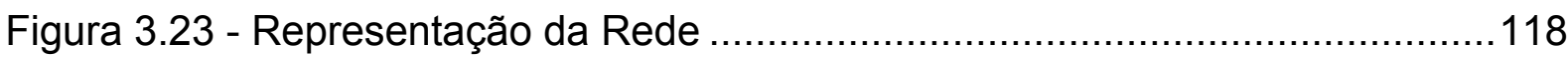

Figura 4.1 - Resposta transitória típica do regulador de velocidade - GGD ..........123

Figura 4.2 -Máxima aceitação de carga em função do BMEP...............................125

Figura 4.3 - Resposta transitória de velocidade GGD1- rejeição carga R-L ...........128 
Figura 4.4 - Resposta transitória de tensão GGD1 - rejeição carga R-L .............128

Figura 4.5 - Resposta transitória de velocidade GGD1 - aceitação carga .............128

Figura 4.6 - Resposta transitória de tensão GGD1 - aceitação de carga ..............129

Figura 4.7 - Autovalores do Sistema - GGD + Carga nominal R-L ....................131

Figura 4.8 - Fatores de Participação - GGD1+Carga nominal R-L ......................132

Figura 4.9 - Lugar geométrico do $\boldsymbol{\lambda}_{\mathbf{1 0 , 1 1}}$ para variações de P e fp - GGD1 ...........135

Figura 4.10 - Lugar geométrico do $\lambda_{\mathbf{8}, 9}$ para variações de P e fp - GGD1 ............136

Figura 4.11 - Resposta transitória para 15\% de variação na carga - GGD1 .........137

Figura 4.12 - Lugar geométrico do $\lambda_{\mathbf{8 , 9}} \boldsymbol{e} \boldsymbol{\lambda}_{\mathbf{1 0 , 1 1}}$ para variações de $\boldsymbol{R}_{\boldsymbol{p}-\boldsymbol{s}}-\mathrm{GGD1} .138$ Figura 4.13 - Resposta transitória de velocidade para variações de $\boldsymbol{R}_{\boldsymbol{p}-\boldsymbol{s}}-$

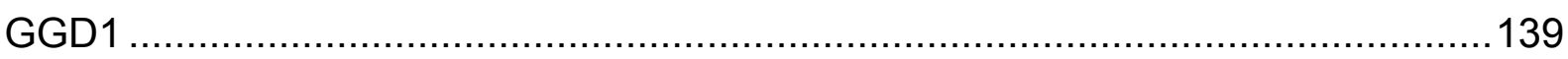

Figura 4.14 - Lugar geométrico do $\lambda_{8,9} e \lambda_{10,11}$ para variações de $K_{i m-S}-$

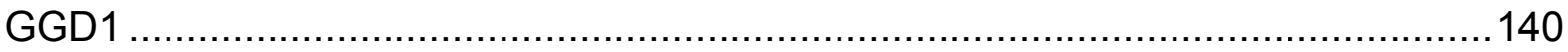

Figura 4.15 - Autovalores do Sistema - GGD + MI .................................... 142

Figura 4.16 - Fatores de Participação - GGD1+MI1 ....................................143

Figura 4.17 - Lugar geométrico do $\boldsymbol{\lambda}_{\mathbf{1 1 , 1 2}}$ e $\boldsymbol{\lambda}_{\mathbf{1 3 , 1 4}}$ para variações no Torque..........144

Figura 4.18 - Resposta transitória para $15 \%$ de variação no Torque -

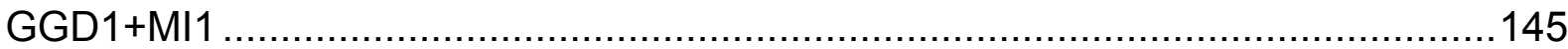

Figura 4.19 - Interface do Inversor - Filtro LC + Indutor de acoplamento .............147

Figura 4.20 - Fluxograma para otimização da interface do Inversor.....................151

Figura 4.21 - Resposta transitória de tensão - Inversor Droop ............................152

Figura 4.22 - Resposta transitória de tensão $I N V 1_{D}$ - Rejeição de carga .............154

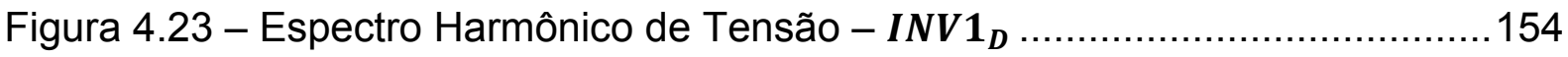

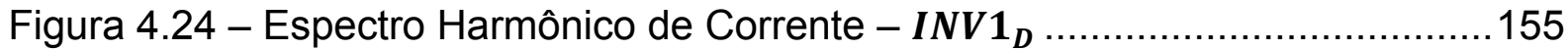

Figura 4.25 - Autovalores do Sistema - $\boldsymbol{I N V}_{\boldsymbol{D}}+$ Carga R-L ...........................156

Figura 4.26 - Fatores de Participação - INV1 + + Carga nominal R-L...................157

Figura 4.27 - Lugar geométrico do $\lambda_{11,12}$ para variações de $\mathrm{P}$ e fp $-\boldsymbol{I N} \boldsymbol{V}_{1 D} \ldots \ldots \ldots . . .158$

Figura 4.28 - Resposta transitória para $15 \%$ de variação na carga - IN $\boldsymbol{V}_{\mathbf{1 D}} \ldots \ldots \ldots . . . .159$

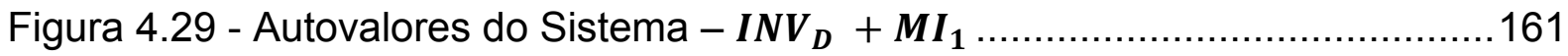

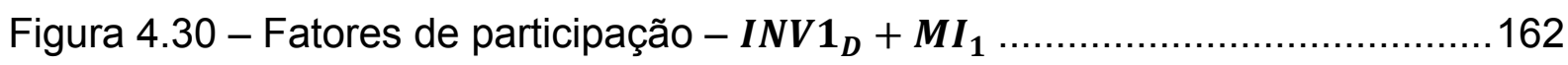

Figura 4.31 - Lugar geométrico do $\lambda_{11,12}, \lambda_{13,14}$ e $\lambda_{17}$ para variações no Torque ....163 Figura 4.32 - Resposta transitória para $15 \%$ de variação no Torque - INV1 $\mathbf{1}_{\boldsymbol{D}}+$ $M_{1}$ 164 
Figura 4.33 - Lugar geométrico do $\lambda_{13}, \lambda_{14} e \lambda_{15}$ para variações de $\mathbf{K}_{\mathbf{i} 0-\mathbf{D}} \ldots \ldots \ldots \ldots . . . .166$

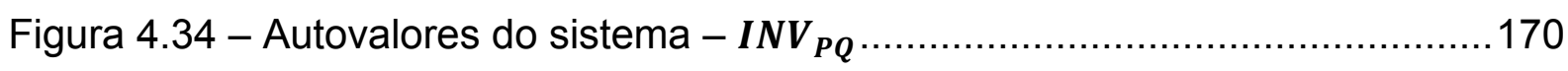

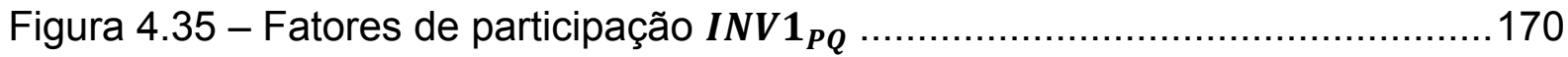

Figura 4.36 - Lugar geométrico do $\lambda_{\mathbf{1 3 , 1 4}}$ para variações de $\mathrm{P}$ e $\mathrm{Q}-\mathbf{I N V} \mathbf{1}_{\boldsymbol{P Q}} \ldots \ldots \ldots . .172$

Figura 4.37 - Lugar geométrico do $\lambda_{15,16}$ para variações de $\mathrm{P}$ e Q $-\mathbf{I N V} \mathbf{1}_{\boldsymbol{P Q}} \ldots \ldots \ldots . . .172$

Figura 4.38 - Resposta transitória para 15\% de variação no $P$ do inversor -

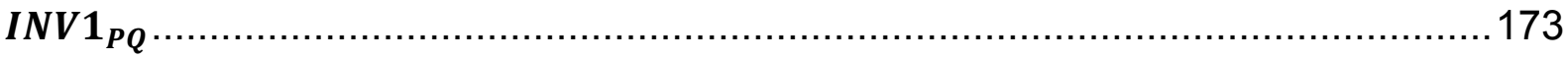

Figura 5.1 - Diagrama unifilar da configuração de MG de teste .........................176

Figura 5.2 - Diagrama Unifilar da MG - Área A ........................................... 177

Figura 5.3 - Autovalores da MG para operação nominal - Área A ........................178

Figura 5.4 - Lugar geométrico do $\lambda_{7,8}$ para variações no $\boldsymbol{P}_{F E D 2}$ e $\boldsymbol{Q}_{F E D 2}$ -

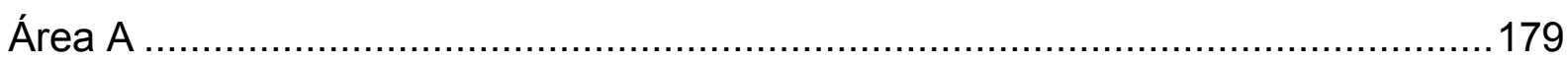

Figura 5.5 - Lugar geométrico do $\lambda_{22,23}$ para variações no $\boldsymbol{P}_{F E D 2}$ e $\boldsymbol{Q}_{F E D 2}-$

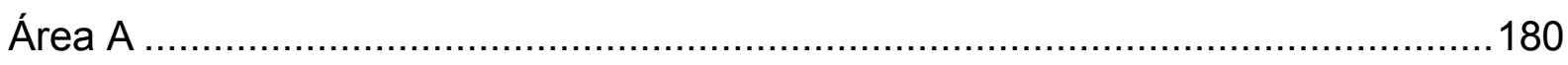

Figura 5.6 - Lugar geométrico do $\lambda_{\mathbf{2 4 , 2 5}}$ para variações no $\boldsymbol{P}_{F E D 2}$ e $\boldsymbol{Q}_{F E D 2}-$

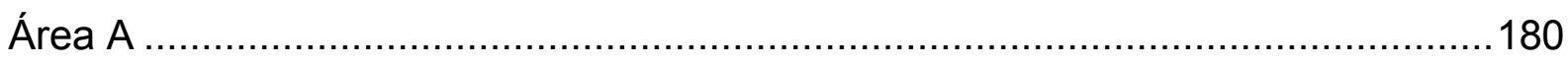

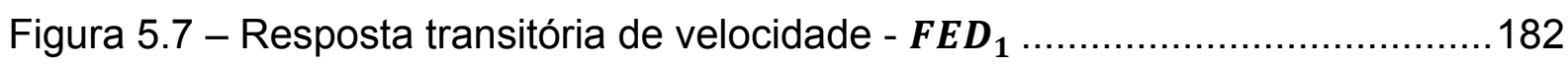

Figura 5.8 - Lugar geométrico dos modos oscilatórios dominantes em função do comprimento do alimentador $C$ - Área A ................................................... 183

Figura 5.9 - Lugar geométrico dos modos oscilatórios dominantes em função da relação R/X do alimentador $C$ - Área A ................................................... 183

Figura 5.10 - Diagrama Unifilar da MG - Área B ........................................ 184

Figura 5.11 - Autovalores da MG para operação nominal - Área B - Controle

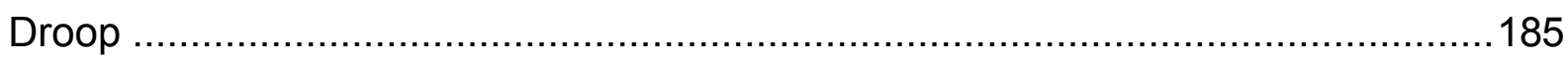

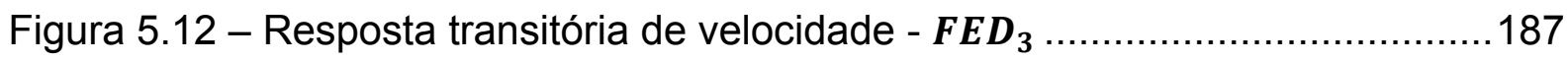

Figura 5.13 - Lugar geométrico do $\lambda_{20,21}$ para variações no $\boldsymbol{P}_{F E D 4}$ e $\boldsymbol{V}_{\mathbf{6}}$ -

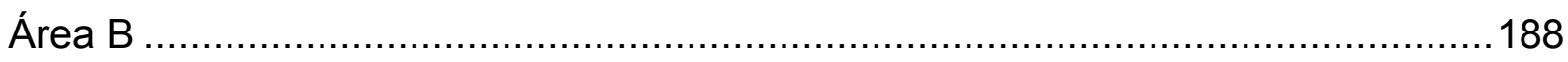

Figura 5.14 - Lugar geométrico do $\lambda_{\mathbf{2 4 , 2 5}}$ para variações no $\boldsymbol{P}_{F E D 4}$ e $\boldsymbol{V}_{\mathbf{6}}-$

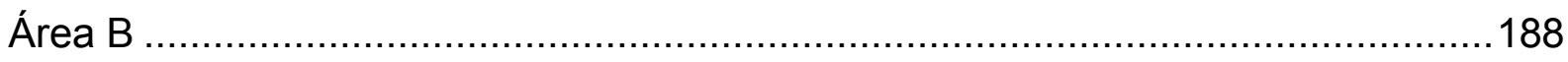

Figura 5.15 - Autovalores da MG na presença do MI - Área B - Controle

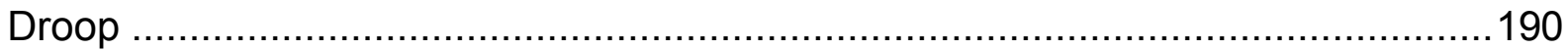

Figura 5.16 - Lugar geométrico dos modos oscilatórios dominantes para variações no Torque... 
Figura 5.17 - Lugar geométrico dos modos oscilatórios dominantes em função do comprimento do alimentador $E$ - Área B.

Figura 5.18 - Lugar geométrico dos modos oscilatórios dominantes em função da relação $\mathrm{R} / \mathrm{X}$ do alimentador $E$ - Área $\mathrm{B}$.

Figura 5.19 - Lugar geométrico dos modos oscilatórios dominantes em função do parâmetro $\boldsymbol{R}_{\boldsymbol{p}-\boldsymbol{D}}-$ Área B 194

Figura 5.20 - Resposta transitória de potência ativa - Área B. 195

Figura 5.21 - Resposta transitória de velocidade $\boldsymbol{F E D}_{\mathbf{3}}$ - Controle Droop+F -

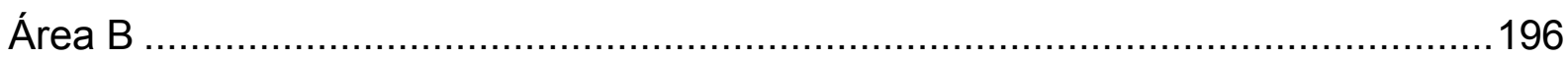

Figura 5.22 - Diagrama Unifilar da MG - Área C ........................................ 197

Figura 5.23- Autovalores da MG para operação nominal - Área C - Controle Droop 198

Figura 5.24 - Lugar geométrico do $\lambda_{\mathbf{2 3 , 2 4}}$ para variações no $\boldsymbol{P}_{F E D 6}$ e $\boldsymbol{Q}_{F E D 6}-$ Área C 199

Figura 5.25 - Lugar geométrico do $\lambda_{25,26}$ para variações no $\boldsymbol{P F E D _ { 6 }}$ e $\boldsymbol{Q F E D _ { 6 }}$ Área C 200

Figura 5.26 - Lugar geométrico dos modos dominantes em função do comprimento do alimentador $G$ - Área C. 201

Figura 5.27 - Lugar geométrico dos modos dominantes em função da relação R/X

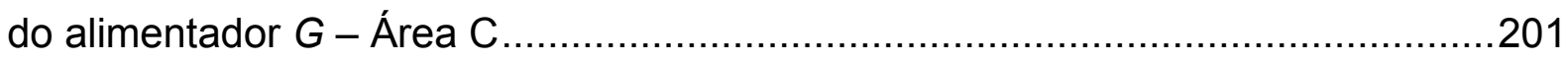

Figura 5.28 - Autovalores do sistema completo - operação nominal ....................204 Figura 5.29 - Lugar geométrico dos modos mais sensíveis às variações no $\boldsymbol{P}_{F E D 2}$ e

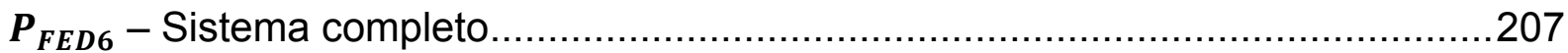

Figura 5.30 - Lugar geométrico dos modos mais sensíveis às variações no $\boldsymbol{Q}_{\boldsymbol{F E D 2} 2}$ e $\boldsymbol{Q}_{\text {FED6 }}$ - Sistema completo 207 


\section{LISTA DE TABELAS}

Tabela 4.1 - Valores limites da resposta transitória - GGDs ................................124

Tabela 4.2 - Parâmetros elétricos e mecânicos dos GGDs ...................................125

Tabela 4.3 - Parâmetros elétricos fundamentais dos GGDs................................126

Tabela 4.4 - Desempenho do sistema GGD+Carga R-L …..............................127

Tabela 4.5 - Ganhos das malhas de controle - GGD.....................................129

Tabela 4.6 - Autovalores do Sistema - GGD + Carga nominal R-L ....................130

Tabela 4.7 - Parâmetros do Motores de Indução ................................................141

Tabela 4.8 - Autovalores do Sistema - GGD + MI ..........................................142

Tabela 4.9 - Limites de distorção de corrente [55] [56] .....................................150

Tabela 4.10 - Limites de distorção de tensão [57] ..............................................150

Tabela 4.11 - Parâmetros dos Inversores - Controle Droop …………………....152

Tabela 4.12 - Ganhos das malhas de controle - Controle Droop ..........................153

Tabela 4.13 - Autovalores do Sistema - $\boldsymbol{I N V}_{\boldsymbol{D}}$ + Carga nominal R-L ...................156

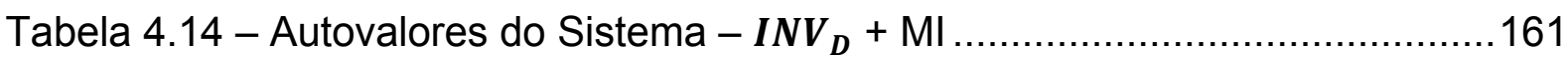

Tabela 4.15 - Ganhos da malha de controle secundário- Controle Droop+F .........165

Tabela 4.16 - Autovalores do Sistema - IN $\boldsymbol{V}_{\boldsymbol{D}+\boldsymbol{F}}+$ Carga nominal R-L.................166

Tabela 4.17 - Parâmetros dos Inversores - Controle P-Q..................................168

Tabela 4.18 - Ganhos das malhas de controle - Controle P-Q ...........................168

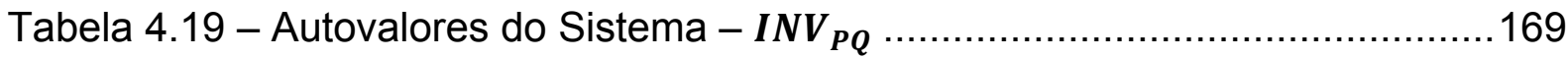

Tabela 5.1 - Autovalores da MG para operação nominal - Área A …....................178

Tabela 5.2 - Autovalores da MG para operação nominal - Área B - Controle

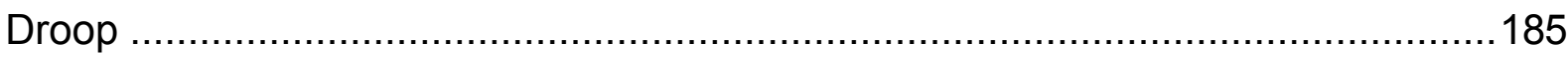

Tabela 5.3 - Autovalores da MG na presença do MI - Área B - Controle Droop ....189 Tabela 5.4-Autovalores da MG para operação nominal - Área B - Controle Droop $+\mathrm{F}$

Tabela 5.5 - Autovalores da MG para operação nominal - Área C - Controle

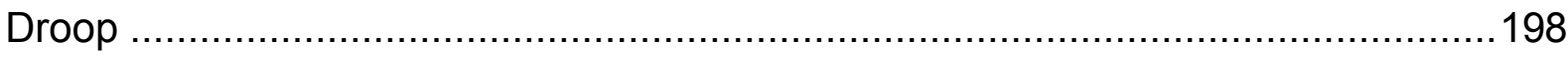

Tabela 5.6 - Autovalores do sistema completo - operação nominal ......................203

Tabela 5.7 - Autovalores do sistema completo - carga leve …...............................205

Tabela 5.8 - Variação do fator de amortecimento dos modos eletromecânicos Sistema em contingência 


\section{LISTA DE ABREVIATURAS E SIGLAS}

\begin{tabular}{ll} 
BMEP & Pressão efetiva média ao freio \\
BT & Baixa tensão. \\
C & Capacitância \\
CC & Corrente alternada. \\
CCMG & Corrente contínua. \\
CHP & Controle Central da Minirrede. \\
D-Q & Geração combinada de calor e energia elétrica. \\
d-q & Sistema de referência rotativo comum \\
EDO & Sistema de referência rotativo local. \\
FC & Equação diferencial ordinária. \\
FED & Função custo ou função objetivo. \\
FPB & Fonte de energia distribuída. \\
fem & Filtro passa-baixa. \\
fp & Inversor com controle P-Q. \\
IND & Força eletromotriz. \\
INGD & Fator de potência. \\
IGBT & Geração distribuída. \\
\hline Inversolated Gate Bipolar Transistor.
\end{tabular}




\begin{tabular}{ll} 
L & Indutância. \\
MI & Minirrede. \\
MT & Motor de indução. \\
P & Média tensão. \\
PCC & Potência ativa. \\
PI & Ponto de conexão comum. \\
PLL & Controlador Proporcional-Integral. \\
PSS & Phase Locked Loop. \\
PWM & Sistemas estabilizadores de potência. \\
Q & Modulação por largura de pulso. \\
R & Potência reativa. \\
THD & Resistência. \\
UCC & Taxa de distorção harmônica. \\
UCM & Unidade de Controle da Carga. \\
UPS & Unidade de Controle do Microgerador. \\
VSC & Fontes ininterruptas de energia. \\
\hline
\end{tabular}




\section{LISTA DOS PRINCIPAIS SÍMBOLOS}

$C_{f}$

$e_{k}$

$e_{k}$

$F_{C-D}$

$F_{c-P Q}$

$F_{v-D}$

$H_{A S}$

$H_{S}$

$i_{c}$

$i_{f}$

$i\left(h_{s w}\right)$

$i_{k}$

$i_{k}$

$K_{A S D}$

$K_{F F}$

$K_{G}$

$K_{i 0-D}$

$K_{i o-P Q}$

$K_{i 1-D}$

$K_{i 1-P Q}$

$K_{i 2-D}$

$K_{i 2-P Q}$

$K_{i m-S}$

Capacitância do filtro LC da interface do inversor com a rede.

Tensão do enrolamento " $k$ " do gerador síncrono com $k=s d, s q, f d, 1 d, 1 q$.

Tensão do enrolamento " $k$ " do motor de indução $\operatorname{com} k=s d, s q, r d, r q$.

Ganho feedforward da malha de corrente do INV $V_{D}$.

Ganho feedforward da malha de corrente do INV $V_{P Q}$.

Ganho feedforward da malha de tensão do INV $V_{D}$.

Constante de inércia do motor de indução [s].

Constante de inércia do GGD [s].

Corrente de saída do inversor.

Corrente no indutor do filtro LC do inversor.

Componente de corrente na frequência de chaveamento do inversor $[A]$

Corrente do enrolamento " $k$ " do gerador síncrono

comk $=s d, s q, f d, 1 d, 1 q$.

Corrente do enrolamento " $k$ " do motor de indução

com $k=s d, s q, r d, r q$.

Coeficiente de atrito do motor de indução.

Ganho pré-feedforward da malha interna de tensão do GGD.

Ganho da realimentação da malha interna de tensão do GGD.

Ganho integral da malha de controle secundário de frequência do

$I N V_{D+F}$.

Ganho integral da malha de controle do PLL do INV $\mathrm{PQ}$.

Ganho integral da malha do controlador de Tensão do INV

Ganho integral da malha do Controlador de Potência do INV $V_{P Q}$.

Ganho integral da malha do controlador de corrente do INV

Ganho integral da malha do Controlador de Corrente do INV $V_{P Q}$.

Ganho integral da malha de controle do regulador de velocidade do GGD. 


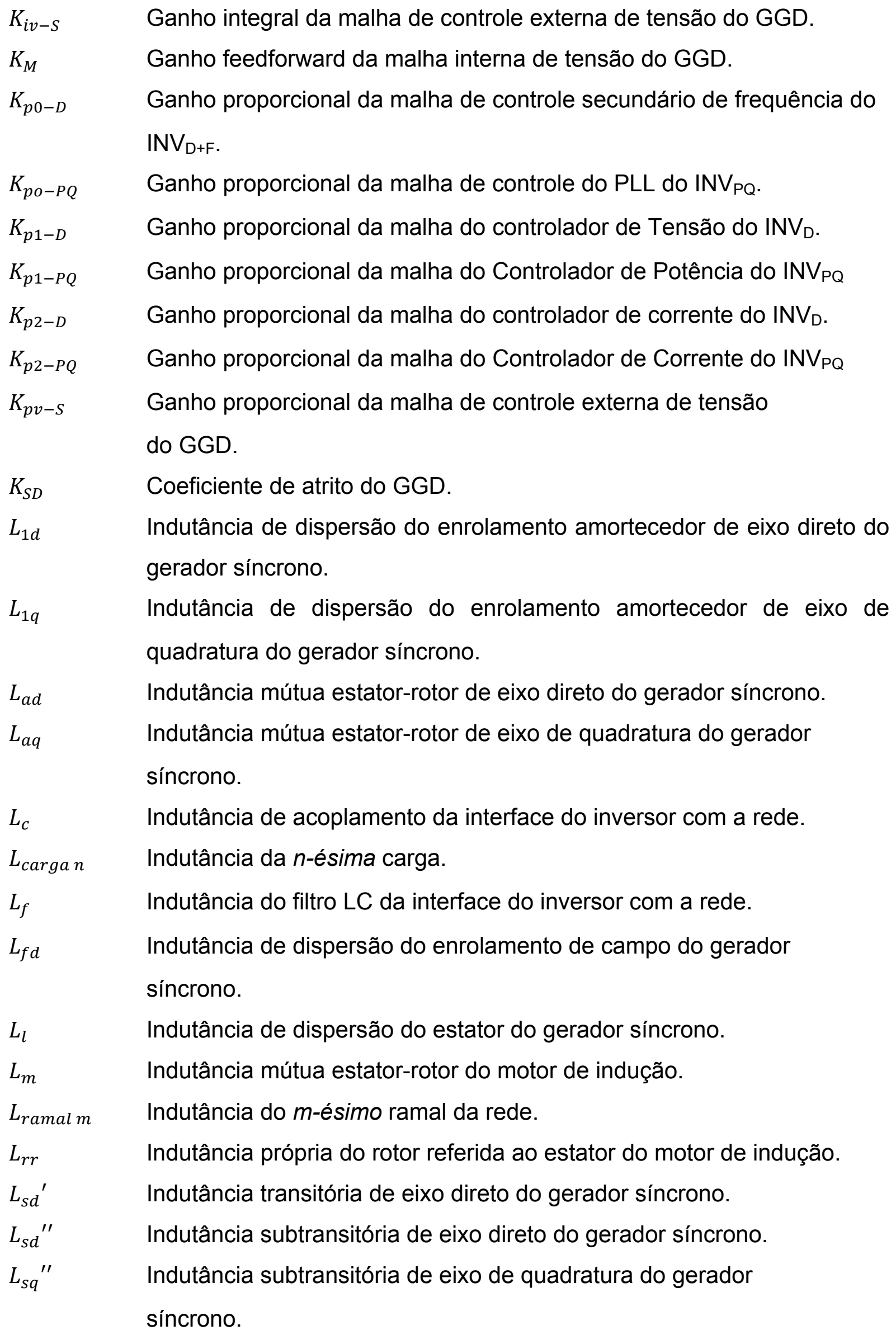




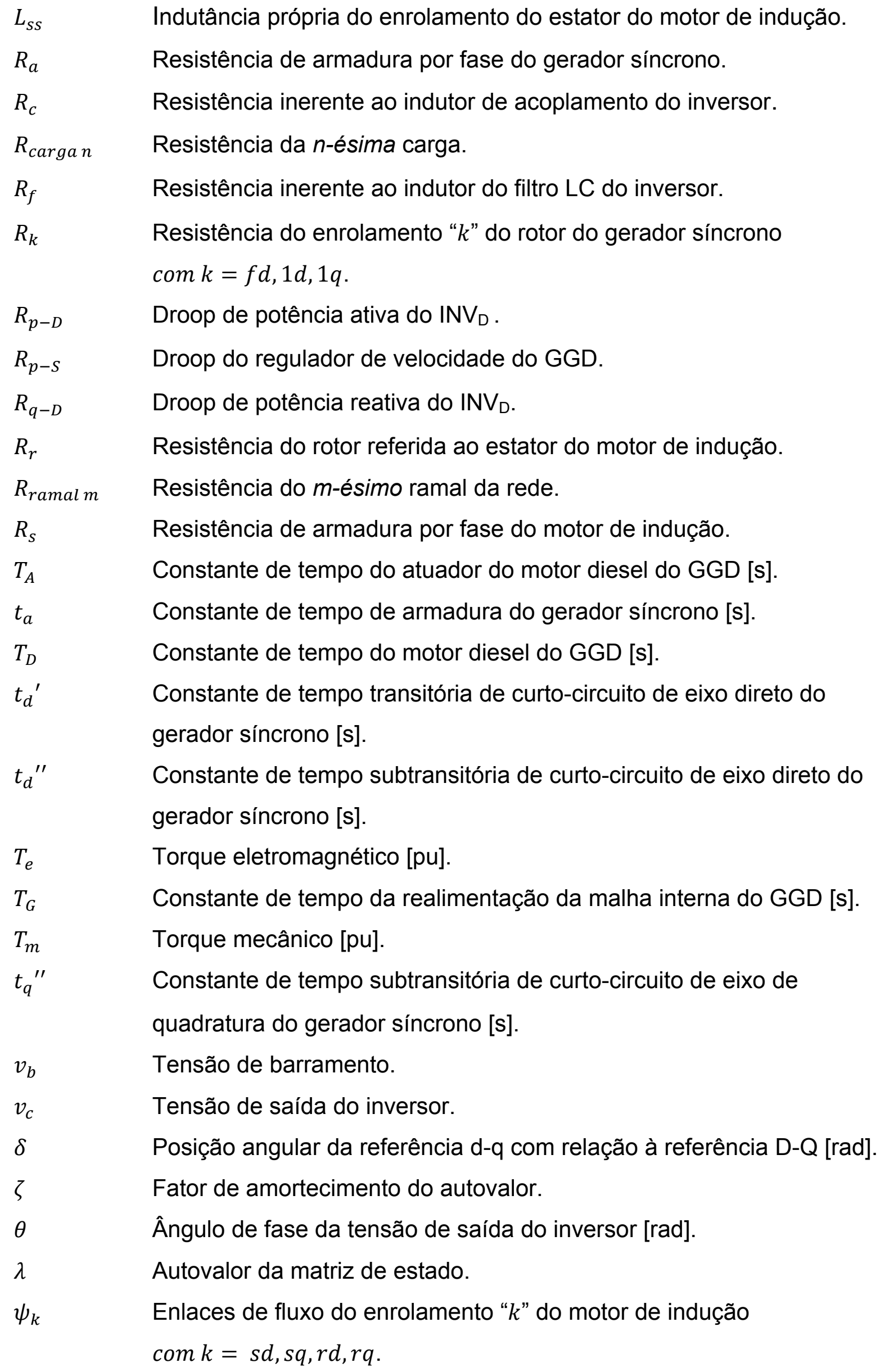


$\psi_{k} \quad$ enlaces de fluxo do enrolamento " $k$ " do gerador síncrono com $k=s d, s q, f d, 1 d, 1 q$.

w velocidade angular da referência d-q.

$w_{\text {base }}$ valor de base da velocidade angular elétrica [rad/s].

$w_{\text {com }}$ velocidade angular da referência comum $D-Q$.

$w_{c}$ frequência de corte do filtro FPB do inversor [rad/s].

$w_{n}$ frequência natural de oscilação do autovalor [rad/s].

$w_{r}$ velocidade angular elétrica do rotor.

$w_{\text {res }}$ frequência de ressonância do filtro LC [rad/s].

$w_{S}$ velocidade síncrona nominal.

$w_{s w}$ frequência de chaveamento do inversor [rad/s]. 


\section{SUMÁRIO}

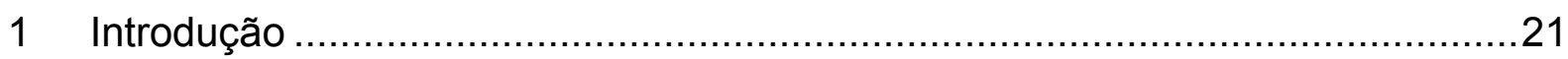

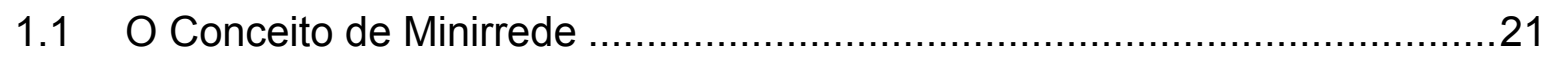

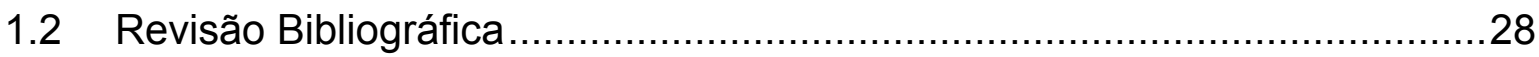

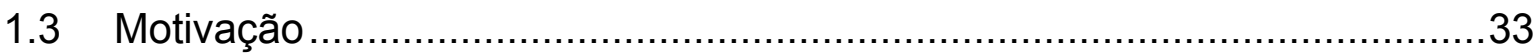

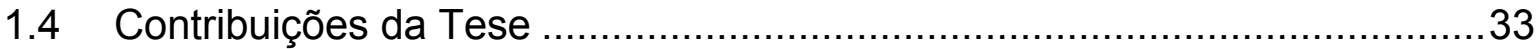

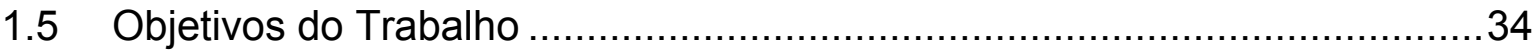

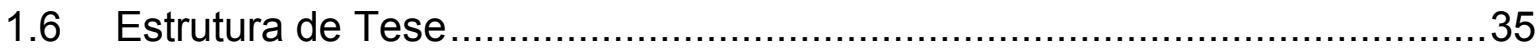

2 Estabilidade de Sistemas Dinâmicos ................................................ 37

2.1 Estabilidade de Sistemas de Potência Convencionais ............................. 37

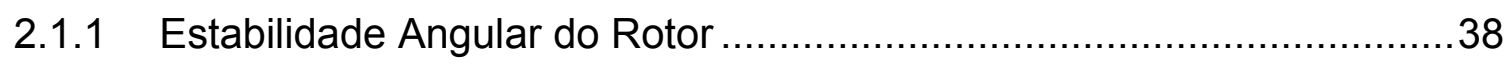

2.1.1.1 Estabilidade Angular a Pequenos Distúrbios ............................39

2.1.1.2 Estabilidade Transitória................................................... 39

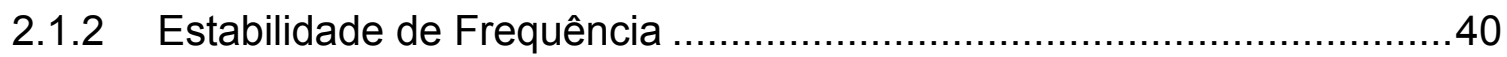

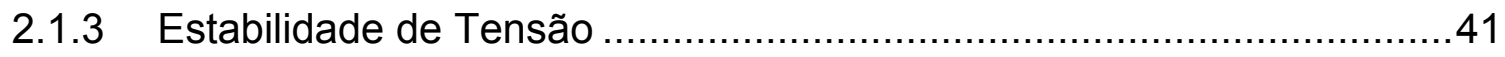

2.1.3.1 Estabilidade de Tensão a Grandes Distúrbios ............................42

2.1.3.2 Estabilidade de Tensão a Pequenos Distúrbios ............................43

2.2 Estabilidade de Minirredes ................................................................. 43

2.3 Conceitos Fundamentais de Estabilidade de Sistemas Dinâmicos .............44

2.3.1 Representação no espaço de estados ......................................44

2.3.2 Estabilidade de um Sistema Dinâmico ......................................46

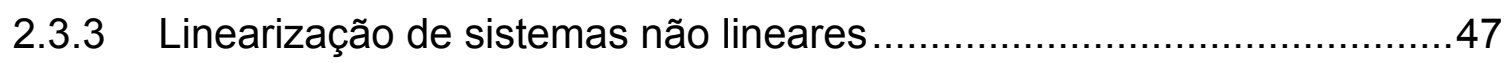

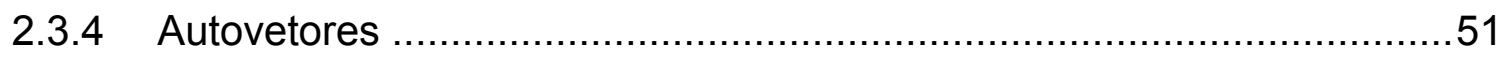

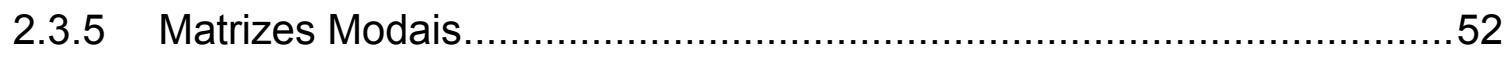

2.3.6 Movimento livre de um Sistema Dinâmico ...................................... 53

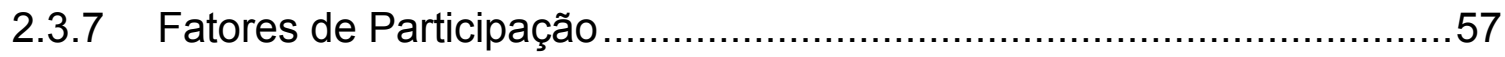


3 Modelagem no Espaço de Estados da Minirrede .58

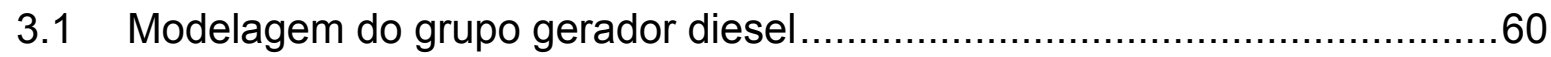

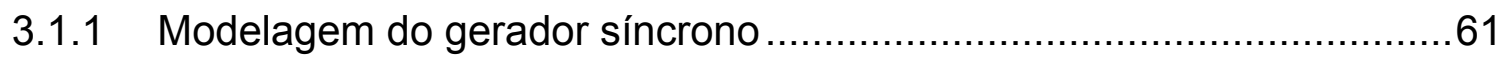

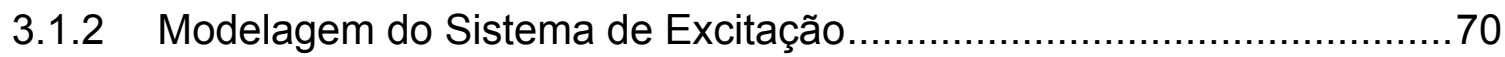

3.1.3 Modelagem do Motor Diesel e do Regulador de Velocidade .................76

3.1.4 Modelo completo do grupo gerador diesel........................................79

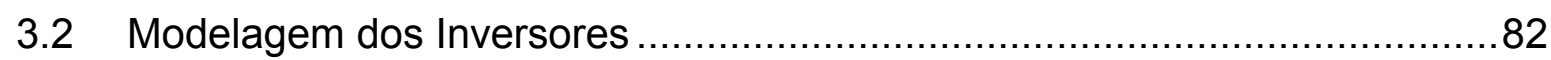

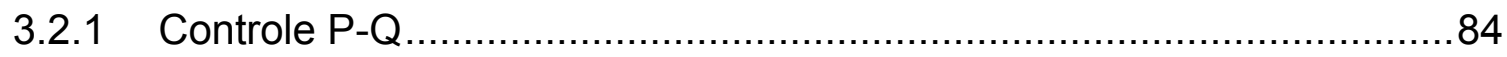

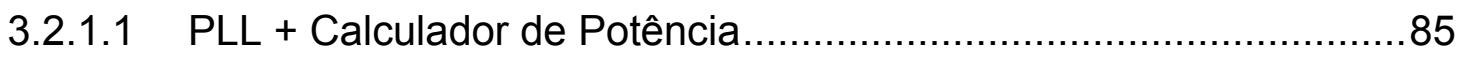

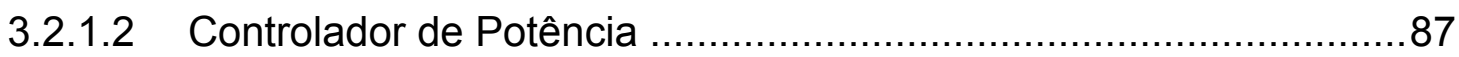

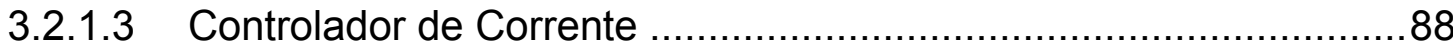

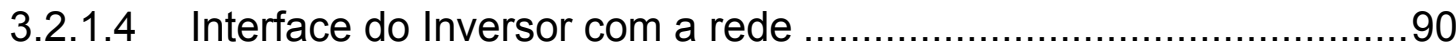

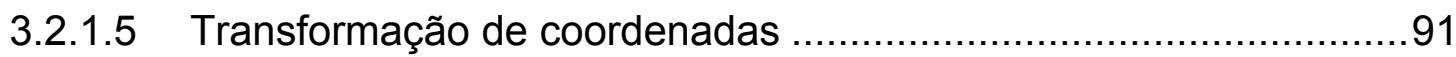

3.2.1.6 Modelo Completo do Inversor P-Q ..............................................

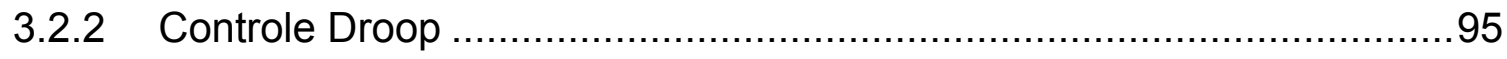

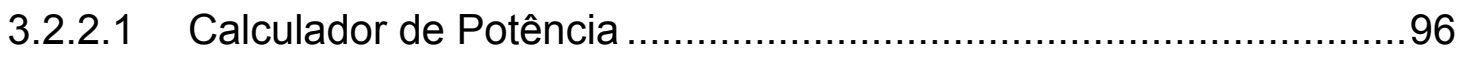

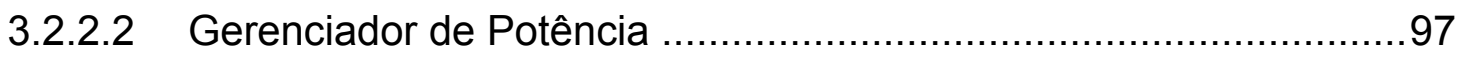

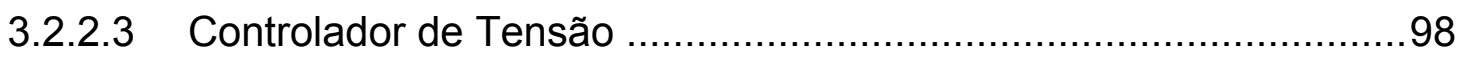

3.2.2.4 Controlador de Corrente ….................................................100

3.2.2.5 Interface do Inversor com a rede ..............................................101

3.2.2.6 Transformação de coordenadas ..............................................102

3.2.2.7 Modelo completo do Inversor Droop …....................................102

3.2.3 Controle Droop com Restauração de Frequência.................................106

3.3 Modelagem das Cargas ……….........................................................

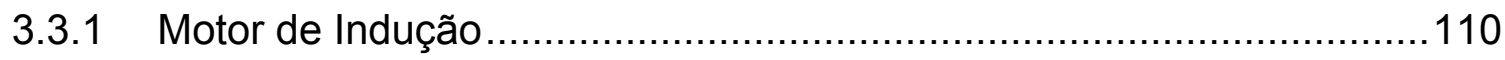

3.3.2 Cargas Passivas de Impedância Constante (R-L) …........................115

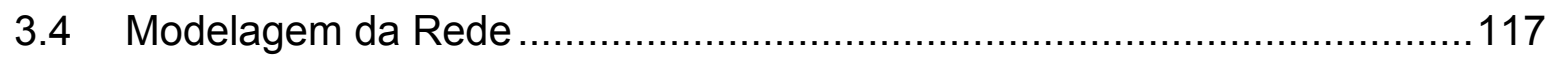


4 Análise da Operação Individual das FEDs ........................................122

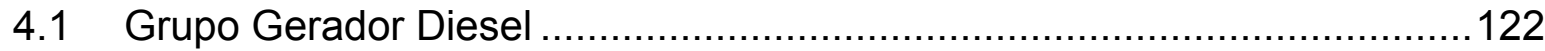

4.1.1 Ajuste das malhas de controle .................................................. 122

4.1.2 Análise da estabilidade dinâmica...............................................130

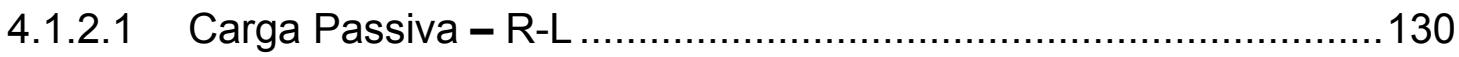

4.1.2.2 Carga dinâmica - Motor de Indução ......................................140

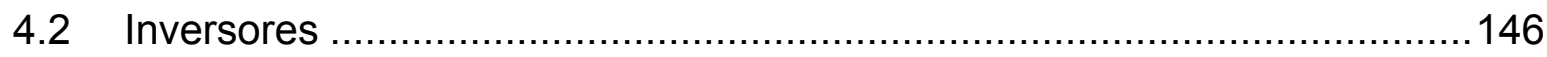

4.2.1 Projeto da Interface do Inversor com a rede ................................146

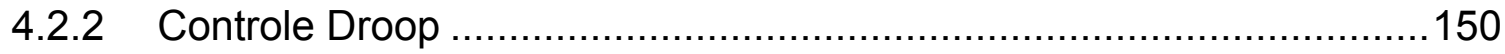

4.2.2.1 Ajuste das malhas de controle .........................................150

4.2.2.2 Análise da estabilidade dinâmica ..........................................155

4.2.3 Inversor Droop com Restauração de Frequência .............................165

4.2.3.1 Ajuste das malhas de controle ........................................... 165

4.2.3.2 Análise da estabilidade dinâmica ..........................................165

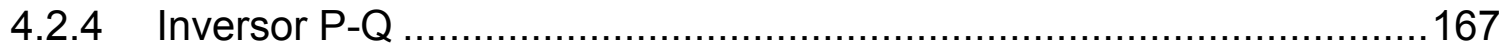

4.2.4.1 Ajuste das malhas de controle ............................................167

4.2.4.2 Análise da Estabilidade Dinâmica ......................................... 169

5 Análise da Operação Conjunta das FEDs na MG ..................................175

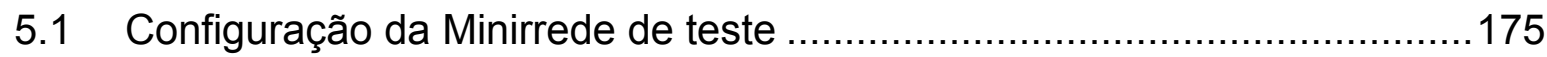

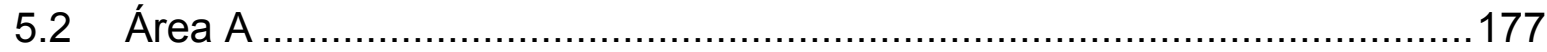

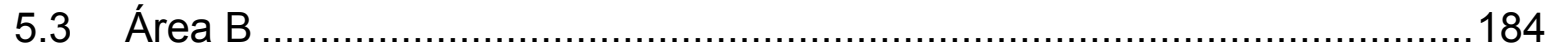

5.3.1 Interface da FED 4 com Controle Droop ......................................185

5.3.2 Interface da $\mathrm{FED}_{4}$ com Controle Droop+F ....................................195

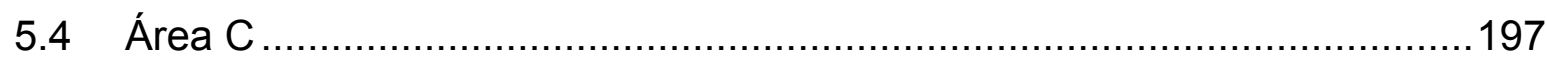

5.4.1 Interface da FED 5 com Controle Droop........................................198

5.4.2 Interface da FED F $_{5}$ com Controle Droop+F ...............................202

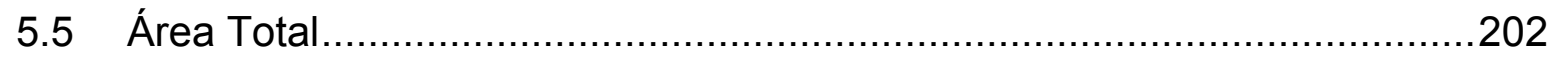


6 Conclusões e sugestões para trabalhos futuros 208

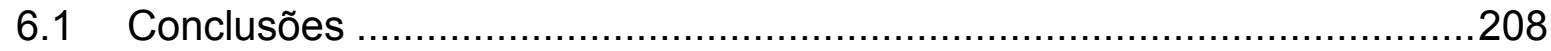

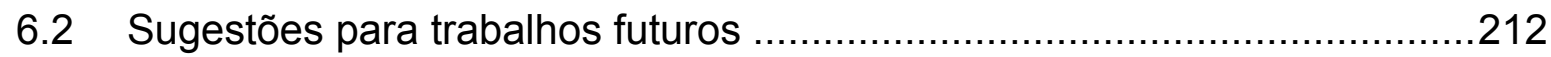

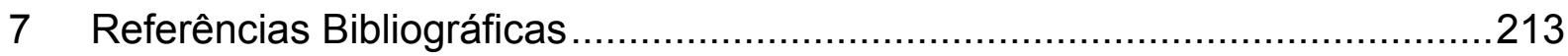

APÊNDICE A

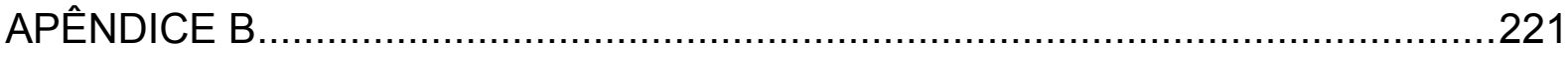




\section{Introdução}

\subsection{Conceito de Minirrede}

Incentivos tecnológicos, econômicos e ambientais estão mudando a forma de produzir e comercializar a energia elétrica no setor brasileiro e no resto do mundo. À medida que as exigências relacionadas à qualidade da energia elétrica fornecida e a proteção do meio ambiente crescem em importância, a geração distribuída (GD) tem aumentado a sua participação no mercado.

Pode-se conceituar geração distribuída, de maneira genérica, como geração de energia localizada junto ou próxima aos consumidores, conectada ao sistema pelo lado do distribuidor ou do consumidor, de pequeno porte e não despachada pelas entidades responsáveis pela coordenação e controle da operação das grandes instalações de geração e transmissão de energia elétrica [1].

As unidades de geração utilizadas na GD, denominadas fontes de energia distribuída (FEDs) ou microgeradores, podem ser convencionais ou alternativas, porém não há consenso sobre o tamanho dessa geração. Alguns autores limitam o tamanho da GD em até $10 \mathrm{MW}$, visto que geradores de maior tamanho são tipicamente interconectados em tensões de transmissão onde o sistema é projetado para acomodar vários geradores [2] [3]. Já outros autores limitam o tamanho da GD em até $100 \mathrm{MW}$ [4].

A GD constitui-se uma boa alternativa às formas convencionais de produção de energia elétrica e vários benefícios têm levado à crescente utilização de GD em diversos países ao redor do mundo. Dentre os benefícios podemos destacar [5] [6]:

- Diversificação da matriz energética.

- Redução dos custos de geração e transporte de energia elétrica.

- Regulação de tensão e redução das perdas técnicas no sistema de distribuição.

- Adiamento de investimentos em reforços da rede para atender o crescimento da demanda. 
- Diminuição dos riscos no planejamento da expansão dos sistemas elétricos devido ao menor tamanho das unidades de geração assim como à flexibilidade das soluções.

- Aproveitamento de combustíveis disponíveis próximos aos centros de carga como gás natural, hidrogênio, álcool, etc.

- Potencial para investimentos em geração combinada de calor e de energia elétrica (CHP), através do uso do calor residual em aplicações industriais, domésticas e comerciais, incrementando notavelmente a eficiência energética total.

No entanto, apesar dos inúmeros benefícios que a GD apresenta para o sistema de distribuição, a indiscriminada conexão amplamente dispersa e não coordenada de FEDs pode trazer mais dificuldades do que soluções. No final da década de 90 , os problemas associados à conexão de GD foram amplamente investigados pelo Comité Técnico do Conselho Internacional de Grandes Sistemas Elétricos (CIGRE) e pela Conferência Internacional sobre Distribuição de Energia Elétrica (CIRED), destacando-se os problemas relacionados à estabilidade, regulação de tensão, conflitos na coordenação das proteções e segurança das equipes de trabalho. Como resultado, restrições técnicas foram impostas à interligação de microgeradores nas redes de distribuição convencionais, destacando-se entre elas, a característica antiilhamento. Recomendações técnicas como a G83/1, G59/1, IEEE 1547, e CEI 11-20, prescrevem a desconexão automática da GD ligada à rede de média tensão (MT) ou baixa tensão (BT) após a saída do circuito alimentador no qual as FEDs são conectadas [2] [7] [8].

Embora os complexos sensores e sistemas de controle existentes permitam realizar uma coordenação centralizada das FEDs com os equipamentos de controle das subestações, isto não resolve totalmente o problema da alta penetração de GD em alimentadores de MT ou BT. A dificuldade com um sistema de controle complexo e centralizado está associada a sua confiabilidade, sendo que uma falha de um único componente ou um erro de software pode provocar um blecaute do sistema ou comprometer a segurança dos operadores das redes de distribuição. Assim, existe a necessidade de que as FEDs sejam capazes de responder às perturbações do sistema de uma forma autônoma, usando unicamente informação local. Por outra 
parte, a característica anti-ilhamento associada às FEDs diminui drasticamente os benefícios que a GD pode aportar ao sistema de distribuição.

Neste contexto, o conceito de Minirrede (MG) é proposto para resolver vários dos problemas associados à integração de microgeradores em alimentadores de distribuição de BT. A MG é uma extensão da GD e pode ser definida como um sistema formado por FEDs, armazenamento e cargas (possivelmente controláveis), gerenciado por um controlador local supervisório com a capacidade de operar de forma paralela ou isolada da rede elétrica convencional [9]. A principal característica distintiva de uma MG é o controle local, que lhe permite operar de forma isolada e autônoma da rede convencional. Durante distúrbios na rede convencional, a MG pode se separar do sistema, isolando as cargas da MG das perturbações, exercendo assim o controle sobre a qualidade e confiabilidade da energia entregue aos usuários finais.

Do ponto de vista da rede convencional, a principal vantagem da MG é que pode ser considerada como uma entidade controlável dentro do sistema de potência, operada como uma única carga agregada com o potencial adicional de participar na prestação de serviços ancilares à concessionária. Já do ponto de vista do usuário, a possibilidade do ilhamento intencional tem a vantagem de proporcionar uma maior confiabilidade local daquela fornecida pelo sistema de distribuição convencional. Por outra parte, ao contrário dos sistemas de distribuição convencionais que fornecem um nível de qualidade de energia uniforme para todos os consumidores dentro de uma determinada área de concessão, as MGs têm o potencial de fornecer qualidade de energia diferenciada, podendo atender facilmente consumidores de alta sensibilidade com menores custos de investimento [7].

Quanto às tecnologias atualmente disponíveis para a microgeração de energia elétrica destacam-se: microturbinas, geradores síncronos ou assíncronos movidos por motores de combustão interna, geração renovável como células de combustível, turbinas eólicas de pequeno porte ou painéis fotovoltaicos. A maioria destas tecnologias gera em corrente continua (CC) ou utilizam geradores rotativos que produzem eletricidade em corrente alternada (CA) com frequência fixa elevada (p.ex. $1800 \mathrm{~Hz}$ no caso da microturbina) ou de frequência variável (no caso da turbina eólica de imã permanente). Em ambos os casos se requer inversores de potência 
(CC/CA ou CA/CC/CA), geralmente do tipo fonte de tensão (VSC), para a interface com a rede de distribuição. Consequentemente, visto que nas MGs não é mandatória a utilização de unidades síncronas que gerem naturalmente em 50 ou 60 $\mathrm{Hz}$ (responsáveis pelo controle da tensão e frequência nos sistemas de potência convencionais), cabe aos inversores associados às FEDs a tarefa de controlar a tensão e frequência no modo de operação ilhada da MG, através de técnicas de controle que emulem a operação da máquina síncrona [10].

Uma configuração típica de MG é mostrada na Figura 1.1. Nela estão indicadas as FEDs, as cargas (assumidas como controláveis) e os dispositivos de armazenamento, bem como os controles e gerenciadores de energia associados ao controle da tensão e frequência. A MG é centralmente controlada e gerenciada pelo Controle Central da Minirrede (CCMG), o qual é instalado no lado de BT da subestação redutora de média tensão. O CCMG desempenha as funções de um controle supervisório garantindo bom desempenho técnico e econômico da MG, com autonomia suficiente para desconectar a MG da rede convencional quando necessário. Para isto, existe um único ponto de conexão da MG com a rede convencional chamado de ponto de conexão comum (PCC). Neste ponto utiliza-se uma Chave de Transferência Estática controlada pelo CCMG, que desempenha um papel importante na interface entre a MG e a rede convencional.

Diferentemente das tradicionais chaves de transferência automática, uma chave de transferência estática proporciona rápida transferência (normalmente $1 / 4$ de ciclo) 0 que assegura operação ininterrupta das cargas sensíveis da MG. A lógica de controle da chave verifica todas as condições de sincronismo antes de conectar a MG na rede convencional, e realiza o ilhamento intencional da mesma quando detectados pelo menos os seguintes tipos de perturbações na rede convencional [11]:

- Faltas no sistema de distribuição a montante do PCC.

- Baixa qualidade de energia: distorção harmônica, cintilação luminosa (flicker) e desequilíbrios de tensão.

- Condição de subfreqüência e/ou subtensão.

- Afundamentos de tensão com tempos de duração superior ao máximo permitido pelas cargas sensíveis da MG. 


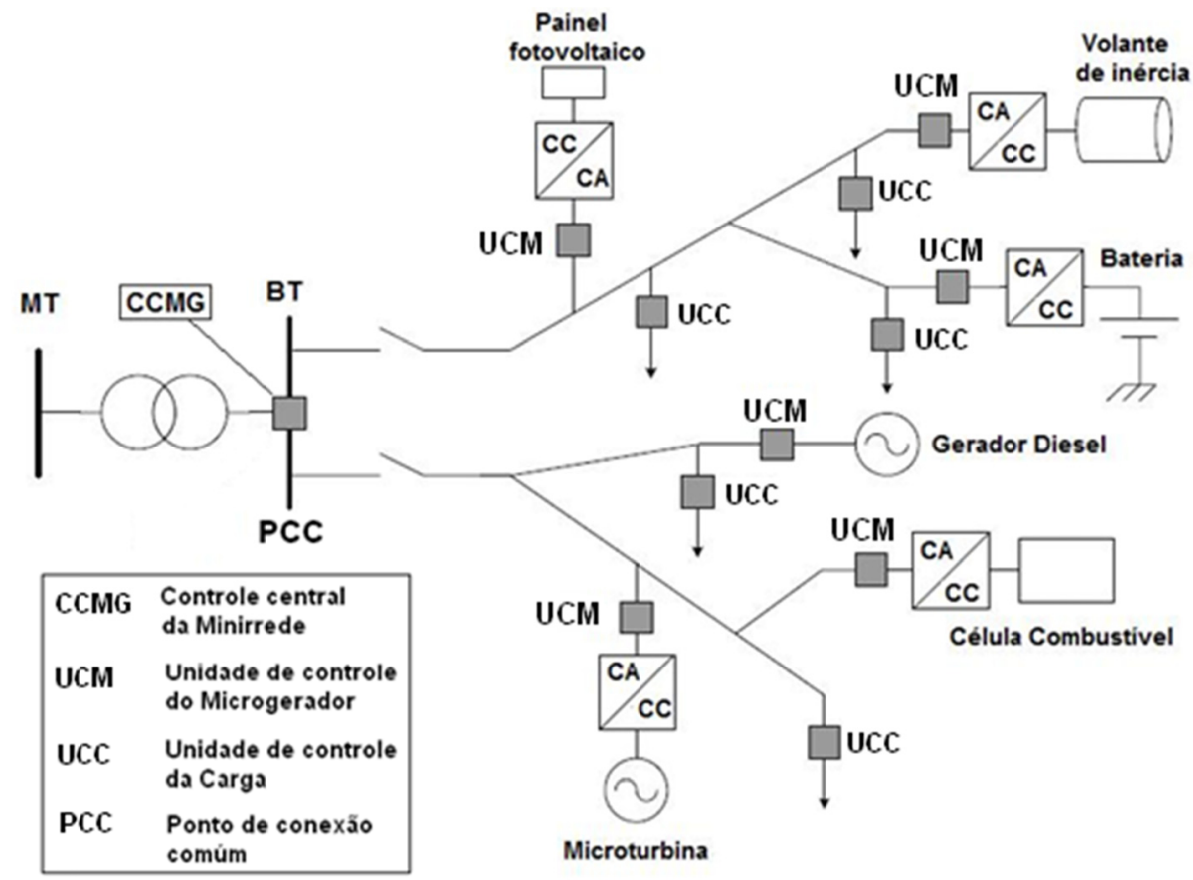

Figura 1.1 - Configuração típica de uma Minirrede [5]

Um segundo nível de controle é estabelecido nas FEDs e nas cargas. $O$ comportamento dinâmico dos Microgeradores é localmente controlado por uma Unidade de Controle do Microgerador (UCM) localizada próxima à FED; e as cargas elétricas (ou grupos de cargas) são controladas por uma Unidade de Controle da Carga (UCC). A quantidade de informação intercambiada entre o CCMG e os demais controladores (UCM e UCC) é mínima, basicamente mensagens de liga/desliga, valores alvos (set-points) relacionados à potência injetada, tensão e frequência, entre outros.

A principal função do UCM é o controle dinâmico independente do fluxo de potência da FED em resposta as variações da carga. Esta característica garante uma rápida tomada de carga por parte do microgerador, especialmente na condição de ilhamento da MG. Esta ação é realizada unicamente através do monitoramento da tensão e corrente local na saída da FED, consequentemente, independe da ação de controle tomada por parte dos UCMs da FEDs vizinhas ou do CCMG. De fato, o UCM tem autonomia suficiente para ignorar as diretrizes de funcionamento providas pelo CCMG que possam colocar em risco a operação da FED associada ao UCM. 
Esta particularidade de controle do UCM, além de garantir maior confiabilidade do sistema, possibilita a introdução de uma nova característica na operação de minirredes, chamada de "ligar e usar" (do inglês plug and play). Sob este enfoque, novas FEDs podem ser adicionadas à MG sem a necessidade de uma engenharia especializada para recalibrar, ou até mesmo reconfigurar, a estratégia de controle das FEDs existentes na MG. Embora esta característica seja chave para o desenvolvimento modular de uma MG, poucos estudos têm sido feitos a respeito da sua influência na estabilidade dinâmica do sistema, sendo esta investigação uma das principais contribuições desta tese.

Já a função do UCC é o gerenciamento da demanda, basicamente através de um controle do tipo liga/desliga. Esta ação permite gerenciar o atendimento às cargas adiáveis e opcionais de tal forma a maximizar a eficiência da MG, assim como preservar a integridade da mesma durante situações de contingência.

A integração de microgeradores dentro de uma MG resulta num sistema com grande número de geradores descentralizados conectados na rede de distribuição interna de $\mathrm{BT}$ da $\mathrm{MG}^{1}$. Para garantir o fornecimento de energia elétrica durante os períodos de ilhamento da MG, os microgeradores não podem depender da rede convencional para seu funcionamento (como acontece atualmente com a GD), portanto eles devem ter a capacidade de contribuir para formação da rede de forma a garantir a funcionalidade e segurança do sistema e manter os valores de frequência e tensão dentro dos níveis aceitáveis. Como mencionado anteriormente, a maioria das FEDs atualmente empregadas em Minirredes utilizam inversores para interface com a rede, assim, estes inversores têm uma influência na frequência e tensão da rede, consequentemente na estabilidade do sistema. Estes inversores, geralmente do tipo VSC modulados por largura de pulso (PWM), empregam chaves autocomutadas de resposta dinâmica rápida, o que dá aos mesmos uma alta flexibilidade de operação.

Dentre as estratégias de controle mais amplamente utilizadas pelos inversores em aplicações de MG destaca-se: (a) Controle P-Q, (b) Controle Droop e (c) Controle Droop com Restauração de Frequência (Droop+F) [12]. Estas três estratégias de controle são descritas brevemente a seguir.

\footnotetext{
${ }^{1}$ No que se segue deste trabalho, a rede de distribuição interna de $B T$ da MG será denominada simplesmente de rede, diferentemente da rede da concessionária denominada como rede convencional.
} 


\section{Controle $P-Q$}

Com este tipo de controle os inversores operam em potência constante, com valores alvos definidos pelo CCMG ou UCM. Neste caso, o inversor é controlado em amplitude e fase emulando uma fonte de corrente controlada, existindo a necessidade de referências de tensão e frequência para sua operação (inversor não autônomo).

\section{Controle Droop}

Este tipo de controle permite ao inversor emular o controle primário de frequência das máquinas síncronas dos grandes sistemas de potência. Nos sistemas convencionais, com o objetivo de permitir a operação em paralelo das unidades geradoras, a característica que governa a velocidade em função da potência de saída de cada unidade tem uma inclinação decrescente (droop de frequência), o que significa que um incremento de carga vem acompanhado de um decremento na velocidade (frequência) da unidade [13].

No caso do inversor, pelo fato das impedâncias entre as unidades de microgeração serem pequenas, existe também a necessidade de implementar um droop de tensão no sistema de controle, o que significa que um incremento de injeção de potência reativa vem acompanhado de um decremento na tensão de saída do inversor. Esta estratégia de controle evita a circulação de grandes correntes reativas entre unidades (eletricamente próximas) que são configuradas com valores alvos de tensão diferentes [11].

Tanto o droop de frequência como o droop de tensão permite que os inversores funcionem de forma autônoma sobre um sistema elétrico isolado, sem a necessidade de um sistema de comunicação entre eles. Este controle também evita a operação mestre/escravo dos inversores que reduz consideravelmente a confiabilidade da MG [14]. 


\section{Controle Droop com Restauração de Frequência}

Este tipo de controle permite ao inversor emular tanto o controle primário como o secundário dos grandes sistemas de potência. O controle secundário nos sistemas convencionais é destinado a restabelecer a frequência do sistema ao seu valor programado [13]. Com a implementação do Controle Droop, o equilíbrio entre a potência gerada e consumida ocorre em uma nova frequência de operação $\left(f_{\text {nova }}=\right.$ $\left.f_{o}+\Delta f\right)$. Sem a atuação de um controle secundário o sistema poderia continuar a operar em uma frequência diferente da programada. O objetivo do Controle Droop com Restauração de Frequência (Controle Droop $+F$ ) é restabelecer a frequência a seu valor nominal (p.ex. $60 \mathrm{~Hz}$ ). Este controle também permite a operação autônoma dos inversores.

\subsection{Revisão Bibliográfica}

O tema Geração Distribuída tem despertado grande interesse em todo o mundo, essencialmente devido a preocupações ambientais e consequentes políticas energéticas assim como também devido a desenvolvimentos tecnológicos em sistemas de geração e controle. Todavia, o conceito de Minirrede é pouco conhecido e explorado, observando-se muitas vezes na literatura que os conceitos de Geração Distribuída, Sistema Hibrido e Minirrede são confundidos e, às vezes, até mesmo tidos por sinônimos.

As principais diferenças entre os conceitos associados à GD e MG foram claramente expostas na Seção 1.1. Os dois conceitos são métodos radicalmente diferentes de instalar e operar FEDs (alternativas e/ou convencionais) conectadas em alimentadores de distribuição. Sob o enfoque da GD os microgeradores são conectados de forma dispersa e descentralizada, sem a capacidade de sustentar ilhas dentro do sistema durante desligamentos da rede convencional. Pelo contrário, sob o enfoque da MG, microgeradores e cargas, são agrupadas dentro de um micro sistema de potência, gerenciado por um controlador local supervisório com a capacidade de operar de forma paralela ou isolada da rede convencional. Como 
resultado, a MG pode ser considerada como uma entidade controlável dentro do sistema de potência de uma concessionária, podendo ser operada como uma única carga agregada, com o potencial adicional de participar na prestação de serviços ancilares.

Já um Sistema Hibrido é definido como qualquer sistema elétrico composto por mais de uma fonte de geração. São sistemas projetados para fornecer energia elétrica às cargas independentes dos grandes e centralizados sistemas interligados de geração. Geralmente são sistemas que contam com a utilização de um grupo gerador diesel (formador de rede) para garantir a regularidade e estabilidade no fornecimento de energia elétrica [15]. Observa-se através desta definição, que Sistemas Híbridos geralmente não são concebidos para trabalhar em sincronismo com a rede convencional.

$\mathrm{Na}$ área da GD muitos autores têm publicado estudos sobre como otimizar a inserção de FEDs ao longo dos alimentadores de distribuição com o intuito de melhorar o perfil de tensão e diminuir as perdas [16] [17] [18]. Estes trabalhos utilizam geralmente procedimentos de otimização baseados em algoritmos genéticos e os resultados obtidos, a partir da metodologia proposta, são comparados com exaustivos fluxos de potência.

Outra questão abordada na área da GD diz respeito a estudos de estabilidade dinâmica e transitória, em alimentadores de distribuição com alta penetração de microgeradores amplamente dispersos. Em [19], um alimentador de distribuição hipotético é simulado assumindo-se um grande número de células combustíveis e microturbinas dispersas ao longo do mesmo. O estudo é realizado considerando uma demanda fixa e sete níveis de penetração da GD. Em cada nível, a estabilidade do alimentador é comparada ao caso base, no qual a carga total é suprida unicamente pelo sistema convencional. Os resultados da comparação permitem avaliar a influência da GD na estabilidade transitória e de tensão da rede convencional.

Em [20], um sistema de distribuição com GD dispersa é modelado no espaço de estados. Todos os microgeradores são do tipo síncrono (diretamente conectados à rede convencional) com exceção da turbina eólica que utiliza gerador de indução de velocidade fixa. Os modelos linearizados dos microgeradores baseiam-se 
unicamente na conhecida equação de oscilação da máquina síncrona que governa a dinâmica rotacional da máquina. Já a turbina eólica é modelada como um sistema dinâmico de duas massas e um único torque de entrada (torque do vento), sendo que o controle do gerador de indução não é modelado. Utilizando-se o enfoque dos autovalores da matriz de estado, uma análise de estabilidade dinâmica é realizada para uma arbitrária configuração do sistema. Baseados nos resultados, mecanismos para melhorar a estabilidade do sistema são propostos.

De modo geral, os trabalhos descritos acima, concentram-se na estabilidade dinâmica e transitória de alimentadores de distribuição com alta penetração de GD. Já na área de Minirredes especificamente, muitos autores tem publicado trabalhos sobre o projeto e controle dos componentes de uma MG. Na sequência serão descritos alguns trabalhos nessa linha.

Em [21] se analisa a aplicabilidade do controle droop em inversores acoplados indutivamente através de alimentadores de BT. O autor mostra o desenvolvimento de um algoritmo de controle baseado nos conceitos anteriormente citados de droop de frequência e tensão. A aplicabilidade do controle se demonstra através de uma experiência de laboratório, onde três inversores monofásicos são conectados através de uma rede de BT para alimentar uma carga, sem nenhum sistema de comunicação entre eles. Os resultados mostram que efetivamente os inversores conseguem emular o controle primário dos grandes sistemas de potência.

Em [22] uma inovação é proposta para ao controle droop, que permite um maior desempenho do sistema no referente a controle de tensão e frequência em redes altamente resistivas. No enfoque clássico do controle droop (controle droop clássico), se assume que o fluxo de potência reativa $(Q)$ entre duas fontes está basicamente relacionado com a diferença de tensão entre elas, enquanto que o fluxo de potência ativa $(P)$ está basicamente relacionado com o ângulo de defasagem das tensões. Isto é verdade em redes puramente indutivas, porém em redes puramente resistivas existe uma inversão desta relação. Assim, em [22] é proposta a utilização de uma matriz de transformação que modifica os valores originais (medidos) de $\mathrm{P}$ e Q para novos valores $\mathrm{P}^{\prime}$ e Q' em função do ângulo de impedância da rede, com a particularidade que estes novos valores sempre mantêm a relação Q-tensão e Pângulo, observada em sistemas puramente indutivos. 
Em [11] se mostra uma visão global do projeto de dimensionamento e controle de uma MG baseada unicamente em inversores autônomos com controle droop clássico. Especial atenção é dada aos requisitos de controle que a chave estática (que serve de interface entre a MG e a rede convencional) deve possuir para permitir uma transição suave (com mínimos transitórios de tensão e frequência) no processo de sincronização da MG com a rede convencional. O dimensionamento do sistema (inversores, indutores de acoplamento, transformadores, alimentadores) e a configuração dos valores de droop dos inversores são baseados unicamente em análises de regime permanente.

Em [23] são analisados o Controle Droop e o Controle Droop $+F$ através de simulações no domínio do tempo. Neste caso utiliza-se um enfoque para as malhas de controle dos inversores baseado no controle vetorial de fluxo (i.e., a integral da tensão de saída do inversor), ao invés do enfoque clássico baseado no controle vetorial de corrente. Uma MG hipotética é simulada no domínio do tempo para testar o desempenho dos controles propostos. Cada tipo de controle é testado individualmente não sendo analisadas possíveis combinações dos mesmos.

Já na área de estabilidade de Minirredes, diversos trabalhos têm sido publicados na ultima década. Estes são detalhados a seguir:

Em [24] se estuda a estabilidade transitória de MGs ilhadas que combinam inversores com controle $\mathrm{P}-\mathrm{Q}$ e droop clássico. Os inversores são modelados sem levar em consideração os efeitos transitórios do chaveamento. Analisam-se duas estratégias de combinação dos inversores. Na primeira, um único inversor com controle droop serve de referência (máster) para a operação em paralelo de todos os outros inversores com controle P-Q. Já na segunda estratégia, dois ou mais inversores são operados com controle droop, sendo os inversores restantes controlados no modo P-Q. Simulações no domínio do tempo são realizadas na plataforma Simulink/Matlab ${ }^{\circledR}$. Uma configuração hipotética de MG formada por quatro microgeradores acoplados através de inversores é utilizada para os testes. Não se analisa a estabilidade dinâmica do sistema simulado.

Em [12] se estuda a estabilidade transitória de MGs sujeitas a perturbações tais como faltas trifásicas e monofásicas. As simulações no domínio do tempo se realizam na plataforma PSCAD/EMTDC. A MG simulada é composta por um 
microgerador e uma fonte de armazenamento (volante de inércia), ambos acoplados à rede através de VSCs. O inversor do microgerador sempre é operado com Controle $P-Q$. Já o inversor do volante de inércia é operado com Controle $P-Q$, Controle Droop e Controle Droop $F$ (por separado), nas diferentes simulações. Com base nas simulações, mecanismos para melhorar a estabilidade transitória da MG são propostos.

Em [25] se analisa a estabilidade dinâmica do controle droop Selfsync ${ }^{T M}$ implementado no inversor comercial de bateria Sunnylsland ${ }^{T M}$ da empresa SMA. O estudo se realiza no domínio da frequência através da análise de polos e zeros da função de transferência representativa do sistema de controle. O inversor é modelado como uma fonte ideal de CA. As cargas são modeladas como impedâncias constantes. Uma simulação (no domínio do tempo) na plataforma ATPEMTP é realizada para corroborar os modelos matemáticos.

Em [26] se analisa a estabilidade dinâmica de dois inversores autônomos, com controle droop clássico, acoplados através de uma rede de BT. Um modelo no espaço de estados (bastante simplificado) é desenvolvido para cada inversor. O modelo assume um comportamento ideal do inversor, isto é, as malhas de controle de tensão e corrente não são modeladas. Esta suposição é baseada no fato da largura de banda das malhas internas (tensão e corrente) ser bem superior à largura de banda do controle de intercâmbio de potência ativa e reativa (controle droop) que estabelece as referências de tensão e corrente para a malha interna. Assim sendo, se admite que a malha de controle interno acompanha as referências de tensão e corrente perfeitamente, com precisão e rapidez, e portanto não tem qualquer efeito sobre a estabilidade dinâmica do sistema. Outra simplificação do modelo assume que os transitórios associados à rede têm constantes de tempo muito pequenas, podendo ser negligenciados no estudo da estabilidade. Consequentemente, a rede é representada pelas equações algébricas da matriz de admitância nodal (equações fasoriais). As cargas são modeladas como valores constantes de impedância.

Já em [27] e [28] modelos mais detalhados de inversores com controle droop clássico são desenvolvidos no espaço de estados. Os modelos levam em conta as malhas de controle internas (tensão e corrente) e externas (controle droop) dos inversores, porém a ação de chaveamento é negligenciada. As cargas são 
modeladas como impedâncias constantes. A partir do modelo completo do sistema (inversor+rede+carga) uma análise de estabilidade dinâmica é realizada com ajuda da técnica dos autovalores próprios da matriz de estado. Uma análise de sensibilidade também é apresentada com o intuito de identificar a origem de cada autovalor.

\subsection{Motivação}

Combinações de FEDs acopladas à rede através de inversores com controle $\mathrm{P}-\mathrm{Q}$, Droop ou Droop $+F$, assim como FEDs ligadas diretamente na rede, possibilitam a criação de um vasto leque de arquiteturas de MG. Diante deste aspecto, surge a necessidade de se analisar a estabilidade dinâmica das Minirredes e determinar configurações de geração, parâmetros de ajuste, estratégias de controle e dimensionamento da rede que garantam o bom desempenho dinâmico do sistema, principalmente quando operado de forma ilhada da rede convencional. Explorar este campo e buscar possíveis contribuições advindas do mesmo constitui a principal motivação desta pesquisa.

\subsection{Contribuições da Tese}

A principal contribuição desta tese é a análise da interação entre as estratégias de controle de inversores autônomos e máquinas rotativas sob o enfoque da característica "ligar e usar", pois nenhum dos trabalhos levantados na revisão bibliográfica aborda o problema de estabilidade dinâmica de MGs sob essa ótica.

Especificamente as contribuições desta tese são:

- Análise da estabilidade dinâmica da MG frente a combinações de FEDs acopladas a inversores com diferentes estratégias de controle (Controle $P-Q$, Controle Droop e Controle Droop $+F$ ). 
- Análise da estabilidade dinâmica da MG frente a combinações de FEDs ligadas diretamente na rede (máquinas síncronas rotativas de frequência industrial), com FEDs acopladas através de inversores.

- Influência da conexão de motores de indução (operando como carga dinâmica) na estabilidade dinâmica da MG.

- Influência dos parâmetros elétricos dos alimentadores de distribuição (impedância e relação $R / X$ ) na estabilidade dinâmica da MG.

\subsection{Objetivos do Trabalho}

Este trabalho tem como objetivo principal contribuir na investigação do problema relacionado à estabilidade dinâmica de Minirredes, constituídas por combinações de FEDs com acoplamento direto e através de inversores. O estudo focaliza na operação ilhada da MG, sendo que combinações das três estratégias de controle (Controle $P-Q$, Controle Droop e Controle Droop $+F$ ) dos inversores são analisadas. Além disso, máquinas assíncronas (motores de indução) são incorporadas à MG com o intuito de investigar o seu impacto na estabilidade do sistema.

A análise realiza-se no espaço de estados com metodologias baseadas nas teorias que envolvem os autovalores e autovetores da matriz de estado, por ser o estado da arte em estudos de estabilidade dinâmica. Modelos no espaço de estados são desenvolvidos para cada componente da MG mantendo um compromisso entre precisão e complexidade. Especial atenção é dada aos modelos dinâmicos das cargas e da rede, da tal forma a garantir sua compatibilidade com os modelos restantes.

O caráter analítico da análise permite investigar a relação entre a estabilidade da MG e os parâmetros do sistema, tais como valores dos droops, dimensionamento da rede, configurações de geração, entre outros.

Especificamente os objetivos do presente trabalho são: 
- Estudo conceitual sobre estabilidade de sistemas de potência.

- Revisão das estratégias de controle para inversores operados em paralelismo sem comunicação.

- Modelagem matemática no espaço de estados das estratégias de controle dos inversores, das máquinas rotativas e demais componentes da MG.

- Investigação do comportamento de múltiplos inversores e máquinas rotativas acopladas através de redes de BT.

- Análises de sensibilidade da estabilidade dinâmica da MG aos parâmetros da rede.

- Testes e validação dos modelos lineares desenvolvidos através de simulações no domínio do tempo na plataforma Simulink/Matlab ${ }^{\circledR}$.

\subsection{Estrutura de Tese}

Esta tese está estruturada em seis capítulos onde se descreve todo o trabalho realizado. O conteúdo é descrito, de forma sucinta, a seguir:

O Capítulo 1 apresenta a contextualização do tema a ser abordado. As técnicas de controle dos inversores para interface das FEDs que não geram naturalmente em frequência industrial são brevemente mencionadas. Em seguida é apresentada a revisão bibliográfica, motivação, contribuições e objetivos da tese.

O Capítulo 2 apresenta o embasamento teórico do trabalho. Assim é realizada uma breve revisão dos tipos de estabilidade comumente estudados em sistemas de potência convencionais e sua aplicabilidade em MGs. Posteriormente, os conceitos fundamentais de estabilidade de sistemas dinâmicos são revistos, introduzindo o conceito de variáveis de estado e modelagem no espaço de estados. Em seguida se mostram as técnicas analíticas utilizadas no estudo da estabilidade dinâmica de sistemas de potência baseadas nos valores e vetores próprios da matriz de estado.

O Capítulo 3 começa com o desenvolvimento do modelo no espaço de estados de um grupo gerador diesel (GGD) equipado com controle de tensão e velocidade, por ser este o tipo de FED (diretamente ligado na rede) mais comumente utilizado em 
MGs. Posteriormente se realiza a modelagem no espaço de estados das três estratégias de controle do inversor. Finalmente, são desenvolvidos modelos no espaço de estados para as cargas (impedância constante e motor de indução) assim como para a rede da MG.

No Capítulo 4 se realiza o ajuste individual das FEDs modeladas no Capítulo 3. As FEDs são calibradas de forma a atender às especificações da resposta transitória segundo Normas. Especial atenção é dada ao projeto da interface do inversor (filtro de saída + indutor de acoplamento) com a rede. Estudos de sensibilidade são realizados com o objetivo de determinar os parâmetros que mais influenciam a operação estável individual das FEDs. Os resultados analíticos são corroborados através de simulações computacionais no domínio do tempo.

No Capítulo 5, uma configuração de MG formada por vários agrupamentos de FEDs conectadas em paralelo através de alimentadores de BT é estudada. A análise individual por agrupamentos de FEDs, assim como estudos de sensibilidade dos autovalores da matriz de estado às variações nos parâmetros da rede, fornecem um panorama completo da relação entre às configurações de MG e a estabilidade dinâmica do sistema, permitindo obter importantes conclusões. Os resultados analíticos são corroborados através de simulações computacionais no domínio do tempo.

Finalmente no Capítulo 6 são apresentadas as conclusões da tese e sugestões para futuros temas de pesquisa. 


\section{Estabilidade de Sistemas Dinâmicos}

\subsection{Estabilidade de Sistemas de Potência Convencionais}

Em termos gerais, a estabilidade de um sistema de potência pode ser definida como sendo a capacidade que este sistema tem de se manter em um estado de equilíbrio, quando em condições operativas normais, e de alcançar um estado de equilíbrio aceitável após ter sido submetido a uma perturbação [29].

A estabilidade de um sistema elétrico de potência é na verdade um problema único, global, onde devem ser considerados os efeitos dos equipamentos tais como: geradores, dispositivos de controle e proteção, linhas de transmissão, elementos de compensação reativa, entre outros. Com facilidade pode se observar quão complexo seria um estudo de tal abrangência. No entanto, fatores como: a severidade dos impactos considerados, a natureza física da instabilidade resultante, o tempo de avaliação e as características dos elementos e processos envolvidos contribuem para uma possível divisão dos estudos de estabilidade.

A Figura 2.1 mostra a classificação da estabilidade para sistemas de potência recomendada pela CIGRE [30].

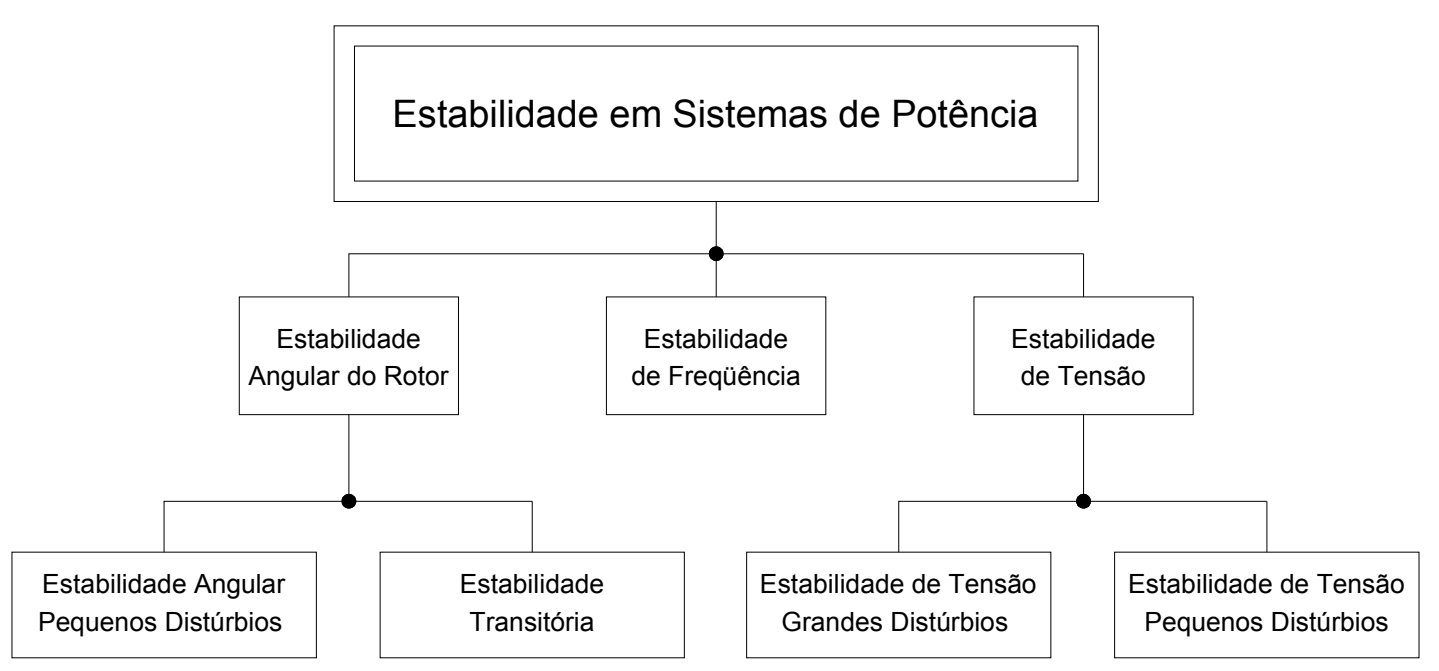

Figura 2.1 - Classificação da estabilidade em Sistemas de Potência 


\subsubsection{Estabilidade Angular do Rotor}

A estabilidade angular do rotor, também conhecida na literatura como estabilidade transitória eletromecânica, está relacionada à capacidade das máquinas síncronas interconectadas permanecerem em sincronismo após um distúrbio qualquer. Estudos deste tipo de estabilidade envolvem as oscilações eletromecânicas, onde um fator importante é a forma em que as potências de saída dos geradores variam à medida que seus rotores oscilam. Enquanto o rotor da máquina oscilar com certo grau de severidade, seja por causa de distúrbios severos ou devido a um simples ajuste no balanço da carga/geração, a potência elétrica de saída da máquina também oscilará. Se o sistema é perturbado, o equilíbrio entre o torque de entrada e o torque eletromecânico de saída se perde, resultando em aceleração ou desaceleração dos rotores das máquinas de acordo com as leis de movimento de um corpo em rotação.

Se um gerador temporariamente gira mais rapidamente que outro, a posição angular do seu rotor relativa ao do rotor da máquina mais lenta se incrementa. Esta diferença angular resultante transfere parte da carga elétrica da máquina mais lenta para a máquina mais rápida dependendo da relação potência-ângulo. Esta ação tende a reduzir a velocidade da máquina mais rápida e consequentemente diminui a separação angular entre elas. Porém, devido à relação potência-ângulo ser altamente não linear, além de certo limite um aumento na separação angular é acompanhado por uma diminuição na potência transferida, isto incrementa a separação angular sem limites e leva o sistema à instabilidade. Para qualquer situação, a estabilidade do sistema dependerá da existência de suficiente separação angular na posição dos rotores, que resulte em suficiente torque restaurador [29]. A configuração do sistema de transmissão e a coordenação de dispositivos de controle e proteção são alguns aspectos que influenciam a capacidade do sistema de suportar os problemas de estabilidade angular.

O fenômeno da estabilidade angular do rotor pode ser caracterizado em termos de categorias permitindo um melhor entendimento da natureza dos problemas de estabilidade. Estas categorias são discutidas a seguir. 


\subsubsection{Estabilidade Angular a Pequenos Distúrbios}

A estabilidade angular a pequenos distúrbios, conhecida também na literatura como Estabilidade Dinâmica ou Estabilidade de Pequenos Sinais ${ }^{2}$, está relacionada à capacidade de um sistema de potência de permanecer em sincronismo durante e após pequenos distúrbios. Tais distúrbios ocorrem frequentemente no sistema devido a pequenas variações entre carga e geração. Os distúrbios são considerados o suficientemente pequenos, de tal forma que a linearização do sistema de equações seja válida para propósito de análise. Linearizar sistemas não lineares é vantajoso, pois permite aplicar numerosos métodos e técnicas de análise linear que proporcionam informações valiosas referentes às características dinâmicas do sistema de potência, auxiliando na operação e projeto do mesmo.

A instabilidade dinâmica que pode acontecer num sistema pode ser de duas formas: (i) aumento permanente do ângulo do rotor devido à ausência de um torque sincronizante suficiente e (ii) aumento na amplitude das oscilações de velocidade do rotor devido à ausência de um torque de amortecimento suficiente. A natureza da resposta do sistema a pequenos distúrbios depende de um conjunto de fatores, incluindo o estado inicial de operação, a robustez do sistema de transmissão e os tipos de controle das máquinas. $\mathrm{O}$ tempo de estudo de interesse na análise de estabilidade dinâmica está na ordem de 10 a 20 segundos após o distúrbio.

\subsubsection{Estabilidade Transitória}

A estabilidade transitória está relacionada com a capacidade de um sistema de potência em manter o sincronismo quando submetido a um distúrbio severo. Estes distúrbios podem ser: curto-circuito (qualquer tipo), perda de importantes unidades de geração, perda de carga, chaveamento de linhas de transmissão, entre outros. A estabilidade depende da condição inicial de operação, do grau de severidade do

\footnotetext{
${ }^{2} \mathrm{Na}$ literatura Norte Americana o termo Estabilidade Dinâmica tem sido utilizado para denotar a Estabilidade Angular a Pequenos Distúrbios de sistemas com presença de dispositivos automáticos de controle, em forma distinta da Estabilidade de Estado Estável, na qual os sistemas não possuem dispositivos automáticos de controle [29].
} 
distúrbio bem como da sua localização. Geralmente, devido à ação dos dispositivos de proteção que atuam para eliminar a falta isolando a área ou o equipamento afetado, a condição de operação pós-perturbação é diferente da pré-perturbação.

A instabilidade transitória que pode acontecer num sistema de potência geralmente tem a forma de uma separação angular aperiódica dos rotores devido à insuficiência do torque sincronizante manifestando-se na primeira oscilação (instabilidade de primeira oscilação). Porém, em grandes sistemas de potência, sistemas estáveis na primeira oscilação podem chegar à instabilidade como resultado de um crescimento oscilatório da separação angular dos rotores. Esta forma de instabilidade geralmente acontece quando a condição de estado estável de pós-falta é por si mesmo instável do ponto de vista da estabilidade dinâmica, é não necessariamente como resultado do distúrbio original [29].

O período de tempo de um estudo de estabilidade transitória é limitado geralmente de 3 a 5 segundos após o distúrbio podendo-se estender até 10 segundos no caso de sistemas de potência de grande porte.

\subsubsection{Estabilidade de Frequência}

Pode-se definir Estabilidade de Frequência como a habilidade do sistema elétrico de potência em manter a frequência dentro da faixa nominal após um distúrbio severo que pode ou não resultar numa divisão do sistema principal em subsistemas (ilhas).

Esta forma de estabilidade depende da habilidade em restaurar o equilíbrio entre geração e carga. A instabilidade acontece como uma oscilação sustentada de frequência que leva à desconexão de geradores e cargas.

Análises de estabilidade de frequência são realizadas via simulações computacionais no domínio do tempo, incluindo dinâmicas rápidas e lentas, associadas aos sistemas de proteção e de controle. As simulações podem incluir distúrbios severos que podem resultar num processo sequencial (efeito cascata) de divisão do sistema em ilhas elétricas isoladas, com os geradores permanecendo em sincronismo. Neste caso, a estabilidade depende da capacidade de cada ilha de 
atingir uma condição de equilíbrio estável com uma perda mínima de carga. A condição de equilíbrio atingida está mais associada à frequência do sistema do que ao movimento relativo dos rotores das máquinas [25].

Geralmente problemas de estabilidade de frequência estão associados à deficiente coordenação dos dispositivos de controle e proteção ou insuficiente reserva de geração. Durante o desenvolvimento de uma condição de instabilidade de frequência, o período de tempo envolvido vai desde poucos segundos, correspondendo às respostas de dispositivos de controle dos geradores e proteções, até dezenas de minutos, correspondendo às respostas dos reguladores de tensão dos barramentos das subestações (p.ex. transformadores com comutação automática de tap sob carga).

A separação de estabilidade de frequência como uma classe distinta permite identificar os eventos iniciais e aplicar ferramentas de análise apropriadas no desenvolvimento de medidas corretivas para problemas de estabilidade não relacionados à tensão ou ao ângulo do rotor.

\subsubsection{Estabilidade de Tensão}

A Estabilidade de Tensão está relacionada com a capacidade de um sistema de potência em manter a tensão dentro dos limites aceitáveis em todas as barras do sistema após ter sido submetido a uma perturbação.

Um sistema é instável do ponto de vista da tensão quando um distúrbio, como um incremento na demanda, causa um progressivo e incontrolável afundamento de tensão. Os fenômenos de estabilidade de tensão estão intrinsecamente ligados ao fluxo de potência reativa no sistema de transmissão, ao comportamento das cargas face às variações de tensão, à ação de dispositivos automáticos de controle de tensão e a limitação de sobre-excitação de geradores.

Um critério para a estabilidade de tensão é que, em uma dada condição operativa para todos os barramentos do sistema, a magnitude de tensão incrementa quando a injeção de potência reativa, no mesmo barramento, também incrementa. Um sistema 
é instável em tensão se, pelo menos em um barramento, a magnitude de tensão decresce quando a potência reativa injetada no mesmo barramento se incrementa [29].

É importante destacar que quedas progressivas de tensão podem também estar associadas com a perda de sincronismo entre as máquinas. Contudo, podem ocorrer situações de instabilidade de tensão onde o comportamento eletromecânico das máquinas síncronas é estável.

A instabilidade de tensão é essencialmente um fenômeno local, porém suas consequências podem ter um impacto generalizado. Já o termo colapso de tensão é mais complexo que uma simples instabilidade de tensão, e usualmente é o resultado de uma sequência de eventos que acompanham a instabilidade de tensão e levam a um blecaute de uma parte significativa do sistema. Instabilidade de tensão nem sempre ocorre isoladamente. Em algumas situações, instabilidade angular e de tensão são problemas relacionados entre si, onde uma distinção entre estes fenômenos pode não estar aparente. Entretanto, é importante distinguir estas formas de instabilidade a fim de compreender e destacar as causas e consequências do problema para desenvolver medidas preventivas/corretivas eficientes.

Para propósitos de análise é útil classificar a estabilidade de tensão em duas subclasses apresentadas a seguir.

\subsubsection{Estabilidade de Tensão a Grandes Distúrbios}

Esta relacionada com a capacidade de um sistema de potência em controlar a tensão após distúrbios severos tais como: faltas, perda de importantes unidades de geração ou contingências de circuitos. Esta capacidade está determinada pelas características da carga e as interações entre os dispositivos de controle e proteção.

A instabilidade de tensão pode envolver uma lenta recuperação dos níveis de tensão e de carga através da ação de dispositivos automáticos de controle de tensão, dispositivos automáticos de recuperação do nível de carga (p. ex. termostatos), ação dos limitadores de sobre-excitação, compensadores síncronos e/ou estáticos 
atingindo seus limites de corrente. Ações manuais executadas pelos operadores ou pelos consumidores também são aspectos importantes. O tempo de estudo de interesse na análise de estabilidade de tensão a grandes distúrbios esta na ordem de 0,5 a 30 minutos após um distúrbio.

\subsubsection{Estabilidade de Tensão a Pequenos Distúrbios}

Esta associada à capacidade do sistema de potência em controlar a tensão após pequenas perturbações tais como incrementos de carga. O processo básico que contribui para este tipo de instabilidade é essencialmente de natureza estacionária. Portanto, análises em regime permanente podem ser utilizadas para determinar as margens de estabilidade do sistema e a identificação dos fatores que influenciam a mesma [29].

\subsection{Estabilidade de Minirredes}

Os problemas de estabilidade descritos acima também acontecem em Minirredes e são objetos de estudos. Porém, tendo em vista a abrangência do estudo, esta tese se limita a análise da Estabilidade Angular do Rotor a Pequenos Distúrbios ${ }^{3}$, de MGs ilhadas. Embora inversores não possuam rotor, a característica potência-ângulo de um inversor com Controle Droop ou Controle Droop $F$ é similar à de um gerador síncrono rotativo, portanto os conceitos associados à Estabilidade Angular do Rotor, revistos nas seções anteriores, podem ser utilizados no caso de microgeradores acoplados à rede através destes tipos de inversores.

Apesar das Minirredes serem sistemas elétricos relativamente pequenos, ainda são sistemas complexos de se analisar (múltiplas entradas e múltiplas saídas). Consequentemente, para facilitar a análise de estabilidade dinâmica, nesta tese

\footnotetext{
${ }^{3}$ No que se segue deste trabalho se utilizará o termo Estabilidade Dinâmica para denotar a Estabilidade Angular a Pequenos Distúrbios, por ser um termo amplamente utilizado na literatura especializada.
} 
utiliza-se o enfoque da teoria de controle moderna, centrada no conceito de espaço de estados, como descrito a seguir.

\subsection{Conceitos Fundamentais de Estabilidade de Sistemas Dinâmicos}

\subsubsection{Representação no espaço de estados}

O comportamento de um sistema dinâmico, tal como um sistema de potência, formado por uma quantidade finita de elementos de parâmetros concentrados, pode ser representado por uma equação diferencial ordinária (EDO) de ordem $n$. Com ajuda da notação vetorial - matricial esta EDO de ordem $n$ pode ser expressa por $n$ equações diferencias ordinárias de $1^{\text {a }}$ ordem da forma [29]:

$$
\dot{x}=f(x, u, t)
$$

onde:

$$
x=\left[\begin{array}{c}
x_{1} \\
x_{2} \\
\cdot \\
\cdot \\
x_{n}
\end{array}\right]
$$

$$
u=\left[\begin{array}{c}
u_{1} \\
u_{2} \\
\cdot \\
\cdot \\
u_{n}
\end{array}\right]
$$$$
f=\left[\begin{array}{c}
f_{1} \\
f_{2} \\
\cdot \\
\cdot \\
f_{n}
\end{array}\right]
$$

O vetor $x$ é chamado de vetor de estado, e seus elementos $x_{i}$ são as variáveis de estado. O vetor $u$ representa os sinais de entrada externos que influenciam a resposta do sistema. A variável $t$ representa o tempo e a derivada da variável de estado $x$ com relação ao tempo é denotada como $\dot{x}$. O vetor $f$ representa um conjunto de funções não lineares relacionando as variáveis de estado, entradas e tempo, às derivadas das variáveis de estado. Se as derivadas das variáveis de 
estado não são funções explicitas do tempo, o sistema é denominado como autônomo e a eq.(2.1) pode ser simplificada para:

$$
\dot{x}=f(x, u)
$$

As saídas do sistema podem ser calculadas em função das variáveis de estado e dos sinais de entrada da seguinte forma:

$$
y=g(x, u)
$$

onde:

$$
y=\left[\begin{array}{c}
y_{1} \\
y_{2} \\
\cdot \\
\cdot \\
y_{n}
\end{array}\right] \quad g=\left[\begin{array}{c}
g_{1} \\
g_{2} \\
\cdot \\
\cdot \\
g_{n}
\end{array}\right]
$$

O vetor $y$ representa as saídas do sistema e $g$ é um vetor de funções não lineares relacionando as variáveis de estado e entradas, às variáveis de saída. O conjunto mínimo de variáveis de estado, tal que o conhecimento destas variáveis em $t=t_{0}$ conjuntamente com as entradas em $t \geq t_{0}$ determine totalmente o comportamento do sistema para qualquer tempo $t \geq t_{0}$, se denomina estado do sistema, e o espaço $n$-dimensional, cujos eixos de coordenadas correspondem as variáveis de estado é denominado espaço de estado. Um estado qualquer pode ser representado através de um ponto no espaço de estado, e a evolução do comportamento dinâmico de um sistema é representada por uma trajetória no espaço de estado [31].

As variáveis de estado não precisam ser quantidades medidas ou observáveis fisicamente, tais como velocidade, tensão, posição, etc. Variáveis que não representam quantidades físicas e que não são susceptíveis de serem medidas ou observadas também podem ser selecionadas como variáveis de estado. Assim sendo, o conjunto de variáveis de estado de um sistema não é único, porém, não 
significa que o estado de um sistema em um dado tempo não seja único. Qualquer conjunto de variáveis de estado provê a mesma informação relacionada ao sistema, de fato, quando se seleciona um conjunto diferente de variáveis de estado, unicamente se está escolhendo um sistema de coordenadas diferente [31].

\subsubsection{Estabilidade de um Sistema Dinâmico}

Os pontos no espaço de estados onde todas as derivadas das variáveis de estado $\left(\dot{x_{1}}, \dot{x_{2}}, \ldots, \dot{x_{n}}\right)$ são simultaneamente zero, são conhecidos como pontos de equilíbrio ou pontos singulares, e definem uma trajetória com velocidade zero. Nestes pontos o sistema está em repouso, consequentemente todas as variáveis de estado são constantes e invariantes no tempo [30]. Os pontos de equilíbrio devem satisfazer a seguinte equação:

$$
f\left(x_{0}\right)=0
$$

onde $x_{0}$ representa o vetor de estado $x$ avaliado no ponto de equilíbrio. Se as funções $f_{i}(i=1,2, \ldots, n)$ na eq. (2.2) são lineares o sistema é linear e tem um único estado de equilíbrio (sempre que o sistema não seja singular). Já no caso não linear, podem existir múltiplos pontos de equilíbrio isolados. Os pontos de equilíbrio são características importantes do comportamento dos sistemas dinâmicos e, portanto, é possível extrair conclusões sobre a estabilidade a partir da sua natureza. Assim, a estabilidade de um sistema linear é inteiramente independente da entrada, e o estado de um sistema estável, com entrada nula, sempre retorna ao espaço de estado original, independente do estado finito inicial. Em contrapartida, a estabilidade de um sistema não linear depende do tipo e magnitude da entrada, e também do estado inicial [29]. Este fator é levado em conta na definição da estabilidade de sistemas de potência (sistemas não lineares) mostrada na seção 2.1.

$\mathrm{Na}$ teoria de sistemas de controle é também uma prática comum classificar a estabilidade de sistemas não lineares em função da região no espaço de estados nas quais o vetor de estado está contido. Assim é dito que um sistema é localmente 
estável na vizinhança de um determinado ponto de equilíbrio se, quando sujeito a uma pequena perturbação, permanece dentro de uma pequena região ao redor do ponto de equilíbrio. Se, com o passar do tempo, o sistema retorna ao ponto de equilíbrio, ele é dito assintoticamente estável. A estabilidade local (i.e., a estabilidade a pequenos sinais) pode ser estudada linearizando-se as equações não lineares do sistema ao redor do ponto de equilíbrio em questão [31].

\subsubsection{Linearização de sistemas não lineares}

Em geral, os procedimentos para encontrar a solução de problemas que envolvem sistemas não lineares são complexos. Devido à dificuldade matemática associada aos sistemas não lineares, resulta necessário introduzir os sistemas lineares equivalentes no lugar dos não lineares. Tais sistemas lineares equivalentes unicamente são válidos para uma faixa limitada de operação. Uma vez que se aproxima um sistema não linear através de um modelo matemático linear, podem aplicar-se numerosos métodos e técnicas de análise linear.

Existem várias técnicas de linearização. O procedimento de linearização que se apresenta aqui se baseia na expansão da função não linear em séries de Taylor ao redor do ponto de operação e a retenção unicamente do termo linear [29]. Devido ao fato dos termos de ordem superior da expansão em séries de Taylor ser ignorados, os termos não considerados devem ser o suficientemente pequenos, isto é, se considera que as variáveis só se desviam ligeiramente da condição de operação. A seguir se descreve o procedimento para linearizar a eq. (2.2).

Seja $x_{0}$ o vetor de estado inicial e $u_{0}$ o vetor de entradas correspondente ao ponto de equilíbrio ao redor do qual se deseja linearizar um conjunto de EDOs não lineares. Desde que $x_{0}$ e $u_{0}$ satisfazem a eq.(2.4) se tem:

$$
\dot{x_{0}}=f\left(x_{0}, u_{0}\right)=0
$$

Perturbando o sistema a partir do seu estado de equilíbrio tem-se: 


$$
x=x_{0}+\Delta x \quad u=u_{0}+\Delta u
$$

sendo que o símbolo $\Delta$ denota um pequeno desvio. O novo estado também deve satisfazer a eq.(2.2), assim:

$$
\dot{x}=\dot{x_{0}}+\Delta \dot{x}=f\left[\left(x_{0}+\Delta x\right),\left(u_{0}+\Delta u\right)\right]
$$

Como a perturbação é assumida pequena, as funções não lineares $f(x, u)$ podem ser expressas em termos da expansão em séries de Taylor. Com os termos envolvendo segundas e maiores ordens de $\Delta x$ e $\Delta u$ desprezados pode se escrever:

$$
\begin{aligned}
\dot{x}_{\iota}=\dot{x_{\imath 0}}+\Delta \dot{x}_{\iota} & =f_{i}\left(x_{0}, u_{0}\right)+\frac{\partial f_{i}}{\partial x_{1}} \cdot \Delta x_{1}+\cdots+\frac{\partial f_{i}}{\partial x_{n}} \cdot \Delta x_{n}+\frac{\partial f_{i}}{\partial u_{1}} \cdot \Delta u_{1}+\cdots \\
& +\frac{\partial f_{i}}{\partial u_{r}} \cdot \Delta u_{r}
\end{aligned}
$$

Como $\dot{x_{\imath 0}}=f_{i}\left(x_{0}, u_{0}\right)$, tem-se:

$$
\Delta \dot{x}_{\imath}=\frac{\partial f_{i}}{\partial x_{1}} \cdot \Delta x_{1}+\cdots+\frac{\partial f_{i}}{\partial x_{n}} \cdot \Delta x_{n}+\frac{\partial f_{i}}{\partial u_{1}} \cdot \Delta u_{1}+\cdots+\frac{\partial f_{i}}{\partial u_{r}} \cdot \Delta u_{r}
$$

com $i=1,2, \ldots, n$. Procedendo de forma similar para a eq.(2.3):

$$
\Delta y_{j}=\frac{\partial g_{j}}{\partial x_{1}} \cdot \Delta x_{1}+\cdots+\frac{\partial g_{j}}{\partial x_{n}} \cdot \Delta x_{n}+\frac{\partial g_{j}}{\partial u_{1}} \cdot \Delta u_{1}+\cdots+\frac{\partial g_{j}}{\partial u_{r}} \cdot \Delta u_{r}
$$

com $j=1,2, \ldots, m$. Dessa maneira, as formas linearizadas das eq.(2.2) e (2.3) são: 


$$
\Delta \dot{x}=A . \Delta x+B . \Delta u
$$

$$
\Delta y=C . \Delta x+D \cdot \Delta u
$$

sendo,

$$
\begin{aligned}
& A=\left[\begin{array}{ccc}
\frac{\partial f_{1}}{\partial x_{1}} & \cdots & \frac{\partial f_{1}}{\partial x_{n}} \\
\cdots & \cdots & \cdots \\
\frac{\partial f_{n}}{\partial x_{1}} & \cdots & \frac{\partial f_{n}}{\partial x_{n}}
\end{array}\right] \\
& B=\left[\begin{array}{ccc}
\frac{\partial f_{1}}{\partial u_{1}} & \cdots & \frac{\partial f_{1}}{\partial u_{r}} \\
\cdots & \cdots & \cdots \\
\frac{\partial f_{n}}{\partial u_{1}} & \cdots & \frac{\partial f_{n}}{\partial u_{r}}
\end{array}\right] \\
& C=\left[\begin{array}{ccc}
\frac{\partial g_{1}}{\partial x_{1}} & \cdots & \frac{\partial g_{1}}{\partial x_{n}} \\
\cdots & \cdots & \cdots \\
\frac{\partial g_{m}}{\partial x_{1}} & \cdots & \frac{\partial g_{m}}{\partial x_{n}}
\end{array}\right] \\
& D=\left[\begin{array}{ccc}
\frac{\partial g_{1}}{\partial u_{1}} & \cdots & \frac{\partial g_{1}}{\partial u_{r}} \\
\cdots & \cdots & \cdots \\
\frac{\partial g_{m}}{\partial u_{1}} & \cdots & \frac{\partial g_{m}}{\partial u_{r}}
\end{array}\right]
\end{aligned}
$$

As derivadas parciais são avaliadas no ponto de equilíbrio em torno do qual a pequena perturbação está sendo analisada. Nas eq. (2.11) e (2.12),

- A é a matriz de estado de dimensão $n \times n$.

- B é a matriz de entradas de dimensão $n \times r$.

- C é a matriz de saídas de dimensão $m \times n$.

- D é a matriz de realimentação (feedforward) de dimensão $m \times r$.

Tomando a transformada de Laplace das eq. (2.11) e (2.12) se tem:

$$
s . \Delta x(s)-\Delta x(0)=A . \Delta x(s)+B . \Delta u(s)
$$




$$
\Delta y(s)=C . \Delta x(s)+D \cdot \Delta u(s)
$$

Onde $s$ representa a variável complexa. Uma solução formal das equações de estado pode ser obtida resolvendo eq. (2.13) e (2.14) para $\Delta x(s)$ e avaliando $\Delta y(s)$ como segue [31]:

$$
\begin{gathered}
(s . I-A) \cdot \Delta x(s)=\Delta x(0)+B . \Delta u(s) \\
\Delta x(s)=(s . I-A)^{-1} \cdot[\Delta x(0)+B . \Delta u(s)] \\
\Delta x(s)=\frac{\operatorname{adj}(s . I-A)}{\operatorname{det}(s . I-A)} \cdot[\Delta x(0)+B . \Delta u(s)]
\end{gathered}
$$

De maneira similar,

$$
\Delta y(s)=C \cdot \frac{\operatorname{adj}(s . I-A)}{\operatorname{det}(s . I-A)} \cdot[\Delta x(0)+B \cdot \Delta u(s)]+D \cdot \Delta u(s)
$$

Consequentemente os polos de $\Delta x(s)$ e $\Delta y(s)$ são as raízes da eq. (2.19):

$$
\operatorname{det}(s . I-A)=0
$$

Os valores de $s$ que satisfazem a eq. (2.19) são conhecidos como autovalores (eigenvalues) da matriz de estado $A$, e a eq. (2.19) é conhecida como a equação característica da matriz $A$. Em outras palavras, os autovalores de $A$ são idênticos aos polos de $\Delta y(s)$ e a condição para estabilidade a pequenos sinais é dada pela localização dos autovalores no plano complexo como se mostra abaixo [29] [32]: 
- Todos os autovalores no lado esquerdo do eixo j.w: será considerado estável; sua resposta livre será uma oscilação amortecida para polos complexos, ou uma exponencial, também decrescente, quando existir apenas polos sobre o eixo real (parte imaginária igual a zero).

- Todos os autovalores com parte real no lado esquerdo do eixo $j . w$, e pelo menos um par de autovalores sobre o eixo $j . w$ : considerado de estabilidade marginal, e sua resposta será uma oscilação constante.

- Pelo menos um autovalor no lado direito do eixo j.w: será considerado instável; sua resposta livre será uma oscilação crescente para polos complexos, ou uma exponencial, também crescente, quando existir apenas polos sobre o eixo real.

\subsubsection{Autovetores}

Como mencionado anteriormente, os autovalores de uma matriz quadrada $A_{(n \times n)}$ são as raízes da equação característica:

$$
\operatorname{det}(\lambda . I-A)=0
$$

As $n$ soluções não triviais da eq.(2.20) ${ }^{4}\left(\lambda_{1}, \lambda_{2}, \ldots, \lambda_{n}\right)$ são os valores próprios de $A$. Para um determinado autovalor $\lambda_{i}$, o vetor $\phi_{i(n \times 1)}$ que satisfaz a eq. (2.21) é chamado de autovetor direito de $A$, associado ao autovalor $\lambda_{i}$.

$$
\text { A. } \phi_{i}=\lambda_{i} \cdot \phi_{i}
$$

Similarmente o vetor $\psi_{i(1 \times n)}$ que satisfaz a eq. (2.22) é chamado de autovetor esquerdo de $A$, associado ao autovalor $\lambda_{i}$.

\footnotetext{
${ }^{4}$ Note-se que a variável complexa $s$ tem sido substituída pela variável $\lambda$ para utilizar a nomenclatura convencional de autovalor encontrada na literatura especializada.
} 


$$
\psi_{i} \cdot A=\lambda_{i} \cdot \psi_{i}
$$

Os autovetores direito e esquerdo associados a diferentes autovalores são ortogonais, em outras palavras, se $\lambda_{i}$ não é igual a $\lambda_{j}$, tem-se [33]:

$$
\psi_{j} \cdot \phi_{i}=0
$$

Caso contrário, se os autovetores correspondem ao mesmo autovalor, se tem [33]:

$$
\psi_{i} \cdot \phi_{i}=C_{i}
$$

Sendo $C_{i}$ uma constante não nula. Devido à eq. (2.20) ser homogênea, $k . \phi_{i}$ ou $k \cdot \psi_{i}$ (onde $k$ é um escalar) também são soluções da eq. (2.21) e eq. (2.22) respectivamente. Consequentemente, os autovetores direito e esquerdo podem ser normalizados tal que [33]:

$$
\psi_{i} \cdot \phi_{i}=1
$$

\subsubsection{Matrizes Modais}

Para analisar as propriedades associadas aos autovalores e autovetores de uma matriz quadrada $A$, é conveniente introduzir as seguintes matrizes, conhecidas como Matrizes Modais [29]:

$$
\begin{gathered}
\Phi=\left[\begin{array}{llll}
\phi_{1} & \phi_{2} & \ldots & \phi_{n}
\end{array}\right] \\
\Psi=\left[\begin{array}{llll}
\psi_{1}^{T} & \psi_{2}^{T} & \ldots & \psi_{n}^{T}
\end{array}\right]^{T}
\end{gathered}
$$




$$
\Lambda=\left[\begin{array}{cccc}
\lambda_{1} & 0 & \ldots & 0 \\
0 & \lambda_{2} & \ldots & 0 \\
& & & \\
\cdots & \ldots & \ldots & \ldots \\
0 & 0 & 0 & \lambda_{n}
\end{array}\right]
$$

Cada uma das matrizes acima tem dimensão $n \times n$. Em termos destas matrizes, a eq. (2.21) e eq. (2.25) podem ser expressas da seguinte forma:

$$
\text { A. } \Phi=\Phi . \Lambda
$$

$$
\Psi . \Phi=I \quad \Psi=\Phi^{-1}
$$

Portanto da eq. (2.29):

$$
\Phi^{-1} . A . \Phi=\Lambda
$$

\subsubsection{Movimento livre de um Sistema Dinâmico}

Referindo-se a eq. (2.11), o movimento livre de um sistema dinâmico (com entrada nula) é dado por:

$$
\Delta \dot{x}=A . \Delta x
$$

Um conjunto de equações expressas desta forma é frequentemente derivado de considerações físicas do sistema e, frequentemente, não é a melhor forma de estudar o comportamento dinâmico do sistema. O problema encontra-se no fato de que, a mudança numa variável de estado é uma combinação linear das mudanças em todas as outras variáveis de estado do sistema. Assim, como resultado do 
acoplamento entre as variáveis de estado, é difícil isolar os parâmetros que afetam consideravelmente a dinâmica do sistema [30].

Para contornar este problema e eliminar o acoplamento entre as variáveis de estado, considere-se um novo vetor de estado $z$ relacionado ao vetor de estado original $\Delta x$ pela transformação:

$$
\Delta x=\Phi \cdot z
$$

Onde $\Phi$ é a matriz modal definida pela eq. (2.26). Substituindo a expressão acima para $\Delta x$ na eq. (2.32), se tem:

$$
\Phi . \dot{z}=A . \Phi . z
$$

A nova equação de estado pode então ser expressa como:

$$
\dot{z}=\Phi^{-1} . A . \Phi . z \quad \dot{z}=\Lambda . z
$$

A eq. (2.35) é conhecida como a forma modal da equação de estado (2.32), com a importante diferença que $\Lambda$ é uma matriz diagonal, enquanto que $A$, de forma geral, é uma matriz não diagonal. A eq. (2.35) representa um conjunto de $n$ equações diferenciais desacopladas de primeira ordem, cuja solução com relação ao tempo é dada por [30]:

$$
z_{i}(t)=z_{i}(0) \cdot e^{\lambda_{i} \cdot t}
$$

Onde $z_{i}(0)$ são os valores iniciais da variável modal $z_{i}{ }^{5}$.

\footnotetext{
${ }^{5}$ Note-se que $z(t)$ são variáveis complexas enquanto $x(t)$ são reais Neste trabalho $z(t)$ são chamadas variáveis modais e $x(t)$ variáveis de estado. $O$ termo $e^{\lambda_{i} \cdot t}$ será referido como modo.
} 
Referindo-se a eq.(2.33), a resposta do sistema em termos das variáveis de estado originais é dada por:

$$
\Delta x(t)=\Phi \cdot z(t)
$$

Resolvendo para $z(t)$ se tem:

$$
z(t)=\Phi^{-1} \cdot \Delta x(t) \quad z(t)=\Psi \cdot \Delta x(t)
$$

Isto implica que:

$$
z_{i}(t)=\psi_{i} \cdot \Delta x(t)
$$

Expandindo a eq. (2.37):

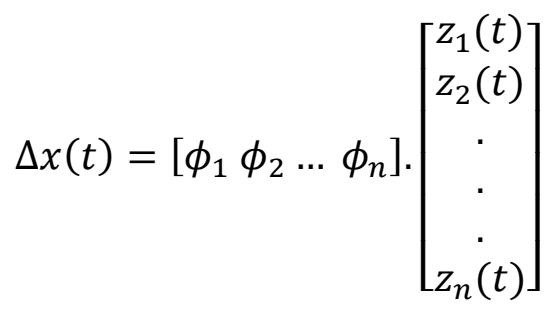

Substituindo a eq. (2.36) na eq. (2.40):

$$
\Delta x(t)=\sum_{i=1}^{n} \phi_{i} \cdot z_{i}(0) \cdot e^{\lambda_{i} \cdot t}
$$

Finalmente substituindo eq.(2.39) na eq. (2.41) se tem:

$$
\Delta x(t)=\sum_{i=1}^{n} \phi_{i} \cdot c_{i} \cdot e^{\lambda_{i} \cdot t}
$$


Onde $c_{i}=\psi_{i} . \Delta x(0)$.representa a magnitude da excitação do i-ésimo modo Em outras palavras, a resposta no domínio do tempo para a i-ésima variável de estado é dada por:

$$
\Delta x_{i}(t)=\phi_{i 1} \cdot c_{1} \cdot e^{\lambda_{1} \cdot t}+\phi_{i 2} \cdot c_{2} \cdot e^{\lambda_{2} \cdot t}+\cdots+\phi_{i n} \cdot c_{n} \cdot e^{\lambda_{n} \cdot t}
$$

A eq. (2.43) fornece uma expressão para o cálculo da resposta livre de um sistema dinâmico, em termos unicamente dos autovalores e autovetores da matriz de estado. A resposta livre (causada pelas condições iniciais) do sistema é uma combinação linear dos $n$ modos dinâmicos correspondentes aos autovalores da matriz de estado.

No caso do sistema possuir autovalores complexos da forma $\lambda=\sigma \pm j$. $w$, a constante $c$ associada a cada termo da eq. (2.43) tem apropriados valores complexos tal que a resposta no tempo $x(t)$ é sempre real [29], por exemplo:

$$
x(t)=(a+j \cdot b) \cdot e^{(\sigma-j \cdot w \cdot t)}+(a-j \cdot b) \cdot e^{(\sigma+j \cdot w \cdot t)}
$$

Onde $c_{1}=a+j . b$ e $c_{2}=a-j . b$. Manipulando a eq.(2.44) é possível eliminar os termos complexos, obtendo-se a seguinte resposta real no domínio do tempo:

$$
x(t)=2 \cdot e^{\sigma \cdot t} \cdot[a \cdot \cos (w \cdot t)+b \cdot \operatorname{sen}(w \cdot t)]
$$

Da eq.(2.45) é fácil concluir que a parte real $(\sigma)$ do autovalor fornece o coeficiente de amortecimento enquanto a parte imaginária $(w)$ fornece a frequência de oscilação associada ao modo. Desta forma, se a parte real é negativa as oscilações são amortecidas. No caso da parte real ser positiva, estas oscilações são caracterizadas por oscilações de amplitude crescente devido à falta de amortecimento 


\subsubsection{Fatores de Participação}

Os fatores de participação definem a participação relativa de uma $k$-ésima variável de estado no i-ésimo modo de oscilação, i.e., quantificam a sensibilidade do i-ésimo autovalor $\left(\lambda_{i}\right)$ ao k-ésimo elemento da diagonal da matriz de estado $\left(a_{k k}\right)$ [30]. Os fatores de participação são quantidades adimensionais que ajudam a identificar as possíveis origens de problemas relacionados com modos oscilatórios pouco amortecidos ou instáveis. O método de cálculo dos fatores de participação se mostra a seguir [29]:

Derivando eq. (2.21) em relação ao elemento $a_{k k}$ da matriz $A$ se tem:

$$
\frac{\partial A}{\partial a_{k k}} \cdot \phi_{i}+A \cdot \frac{\partial \phi_{i}}{\partial a_{k k}}=\frac{\partial \lambda_{i}}{\partial a_{k k}} \cdot \phi_{i}+\lambda_{i} \cdot \frac{\partial \phi_{i}}{\partial a_{k k}}
$$

Pré-multiplicando a eq. (2.46) por $\psi_{i}$ e notando que $\psi_{i}$. $\phi_{i}=1$ e $\psi_{i}\left(A-\lambda_{i} I\right)=0$ se tem:

$$
\psi_{i} \frac{\partial A}{\partial a_{k k}} \cdot \phi_{i}=\frac{\partial \lambda_{i}}{\partial a_{k k}}
$$

Todas as derivadas $\frac{\partial A}{\partial a_{k k}}$ são zero, exceto para o elemento na k-ésima fila e na $k$ ésima coluna, para o qual a derivada é igual a 1. Consequentemente:

$$
\frac{\partial \lambda_{i}}{\partial a_{k k}}=\psi_{i k} \cdot \phi_{k i}
$$

Portanto, a sensibilidade do autovalor $\lambda_{i}$ ao elemento $a_{k k}$ da matriz $A$ é igual ao produto do elemento $\psi_{i k}$ do autovalor esquerdo vezes o elemento $\phi_{k i}$ do autovalor direito. Devido aos autovetores serem costumeiramente normalizados, a soma dos fatores de participação associados com um determinado modo ou com uma determinada variável de estado é igual a 1. 


\section{Modelagem no Espaço de Estados da Minirrede}

A Figura 3.1 ilustra a estrutura simplificada de uma MG. Os elementos que compõem esta MG podem ser divididos em três categorias: fontes, cargas e rede de distribuição interna de BT (denominada neste trabalho simplesmente de rede). Existem vários tipos de FEDs convencionais e/ou alternativas que podem compor uma MG, o que à primeira vista, indicaria a necessidade de desenvolver um modelo dinâmico para cada tipo de gerador elétrico junto com sua fonte primária de energia ${ }^{6}$. Contudo, como visto no Capítulo 1, a maioria das FEDs elétricas utilizadas em MGs não geram naturalmente na frequência de $50 \mathrm{~Hz}$ ou $60 \mathrm{~Hz}$, sendo necessária a utilização de inversores para realizar a interface com a rede. Considerando o intervalo de tempo de interesse nos estudos de estabilidade dinâmica, assim como o fato dos inversores possuírem algum tipo de armazenamento de alta capacidade no elo CC (para garantir o balanço inicial de energia [14] [24]), pode se assumir que o inversor desacopla totalmente o gerador elétrico do resto do sistema, sendo desnecessária a modelagem tanto do gerador elétrico como da sua fonte primária de energia.

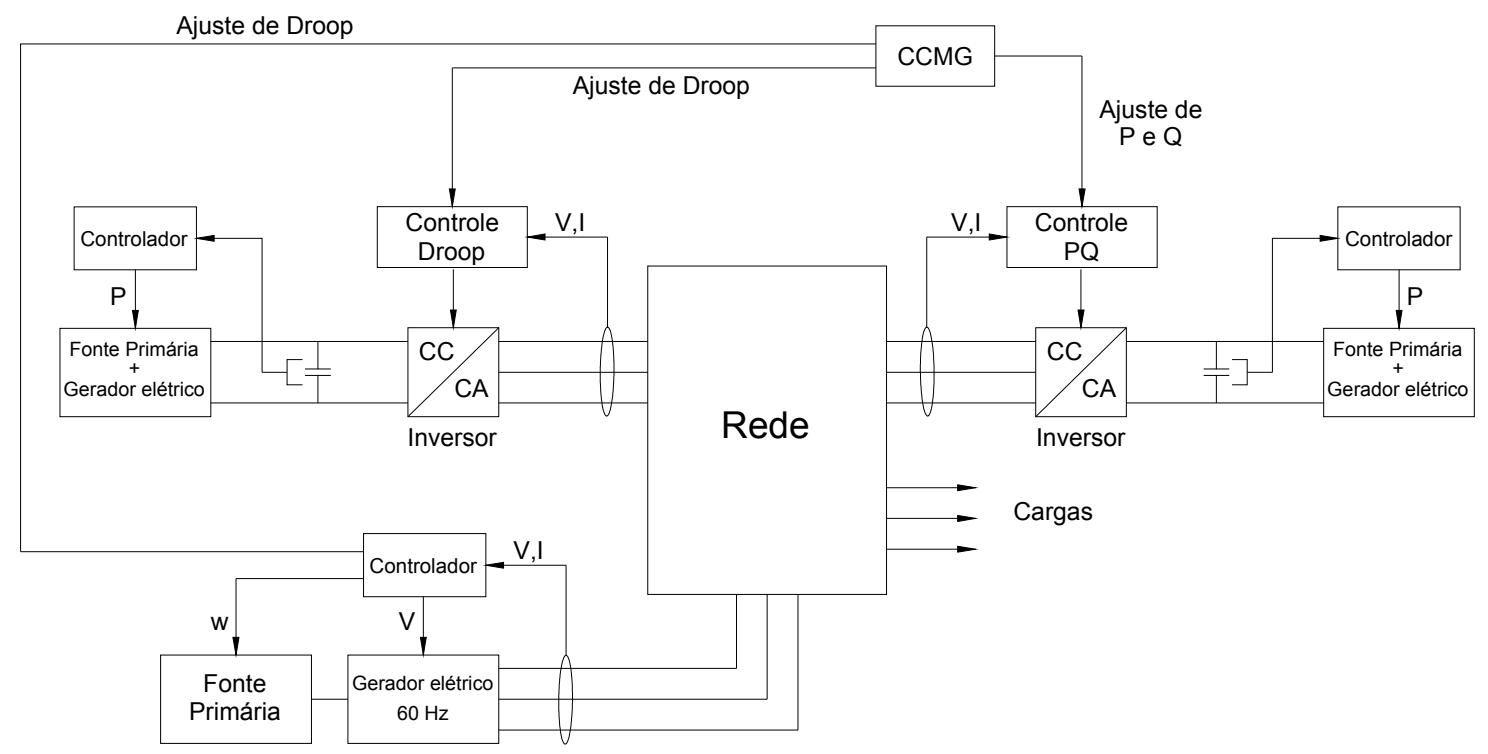

Figura 3.1 - Estrutura simplificada de uma MG

\footnotetext{
${ }^{6}$ Neste caso, utiliza-se o termo fonte primária de energia para descrever todos os componentes à montante do gerador elétrico necessários para a produção de energia elétrica.
} 
Assim sendo, o leque de FEDs que podem compor uma MG (do ponto de vista da modelagem) pode ser dividido em dois grupos: FEDs acopladas à rede através de inversores e FEDs diretamente ligadas à rede. No primeiro caso, existe a necessidade de modelar em detalhe o inversor com suas estratégias de controle, sendo que neste trabalho são modeladas três das principais estratégias de controle comumente utilizadas em MGs. Já para o segundo caso, são modelados unicamente GGDs equipados com gerador síncrono trifásico, por ser este o tipo de FED (diretamente ligada à rede), mais comumente utilizado em MGs.

No referente às cargas, neste trabalho são modeladas cargas dinâmicas (motores trifásicos de indução) e cargas passivas trifásicas de impedância constante (R-L). Especial atenção é dada aos modelos dinâmicos das cargas e da rede, de tal forma a garantir sua compatibilidade com os modelos desenvolvidos para as fontes.

Cada componente da MG é representado no espaço de estados com base nas EDOs que governam seu comportamento dinâmico, desenvolvido na referência rotativa local $(\mathrm{d}-\mathrm{q})$. Entretanto, para modelar a MG como um todo é necessário estabelecer uma referência rotativa comum (D-Q) escolhida arbitrariamente dentre as referências locais $d-q$, assim como um jogo de variáveis elétricas de entrada e saída comuns aos modelos das fontes e das cargas, de tal forma a permitir o acoplamento dos componentes na configuração de MG requerida.

Este processo se representa graficamente na Figura 3.2, onde se observa que arbitrariamente a referência d-q da $F E D_{1}$ é escolhida como referência comum do sistema, fornecendo a velocidade $w_{c o m}$ de sua referência rotativa como base para todos os outros componentes da MG. As variáveis elétricas de entrada correspondem às tensões de fase verificadas no barramento de conexão do componente com a rede, transladadas para a referência comum $\left(v_{b D Q}\right)$, enquanto que as variáveis elétricas de saída correspondem as correntes injetadas pelo componente na rede, também transladadas para a referência comum $\left(i_{D Q}\right)$. 


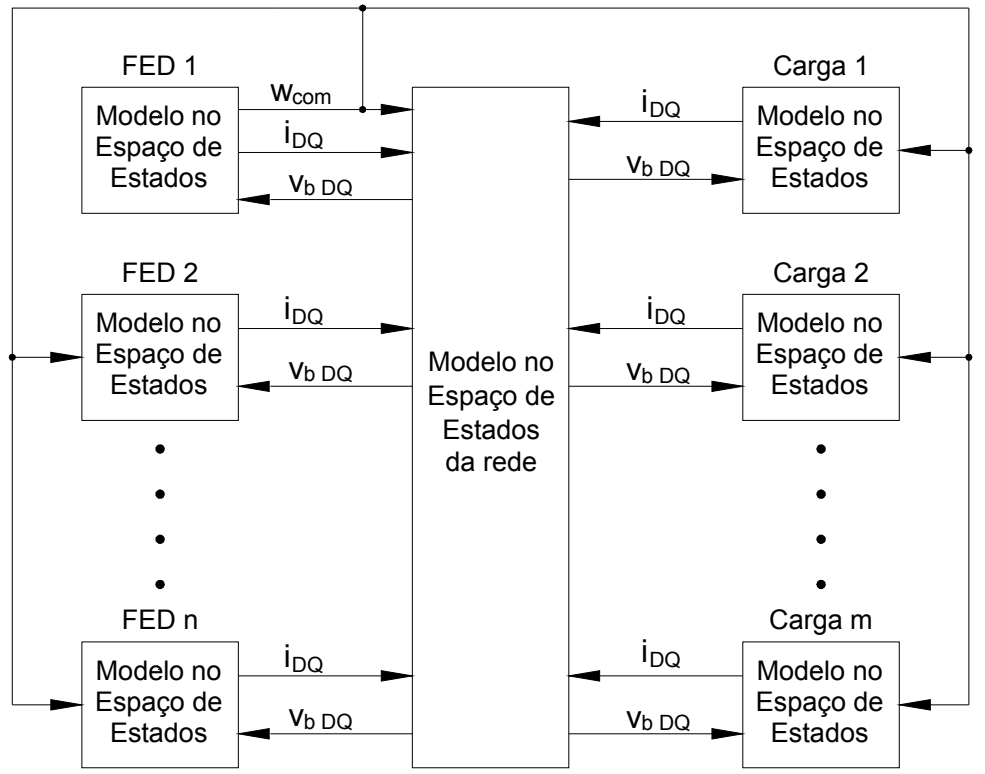

Figura 3.2 - Modelo completo no espaço de estados de uma MG

\subsection{Modelagem do grupo gerador diesel}

Um grupo gerador diesel (GGD) é composto por um motor diesel (fonte primária de energia) diretamente acoplado a um gerador elétrico, que pode ser do tipo síncrono ou assíncrono. Em aplicações de GD, com potências inferiores a $300 \mathrm{~kW}$, é muito comum encontrar GGDs equipados com geradores assíncronos (sem conversores eletrônicos de potência), devido à relativa facilidade em satisfazer os requisitos mínimos das concessionárias para interligação destes geradores com a rede convencional se comparados aos geradores síncronos [2]. Contudo, em sistemas elétricos com a capacidade de operar de forma ilhada da rede convencional (como é o caso das MGs), existe muitas vezes a necessidade de se controlar tensão e frequência do sistema através de um ou vários GGDs, sendo neste caso os geradores síncronos a opção mais utilizada. Neste trabalho será modelado um GGD com gerador síncrono trifásico de polos salientes equipado com controle de excitação e regulador de velocidade, por ser este o tipo de gerador mais comumente utilizado em MGs. Primeiramente será desenvolvido o modelo do gerador elétrico e posteriormente o modelo será expandido de forma a incluir a dinâmica do motor diesel e dos controles de excitação e de velocidade. 


\subsubsection{Modelagem do gerador síncrono}

As equações diferenciais que descrevem o comportamento dinâmico de um gerador síncrono trifásico são desenvolvidas em detalhe em [29] e [34]. Aqui unicamente se mostra a manipulação destas equações de tal forma a se desenvolver um modelo linear no espaço de estados da máquina, compatível com o modelo padrão mostrado na Figura 3.2. Usualmente, os modelos utilizados na representação de máquinas síncronas recaem na utilização de bobinas fictícias representadas sobre uma referência rotativa de eixo direto $(d)$ e quadratura $(q)$ que gira na mesma velocidade elétrica que o rotor da máquina $\left(w_{r}\right)$ como se mostra na Figura 3.3.

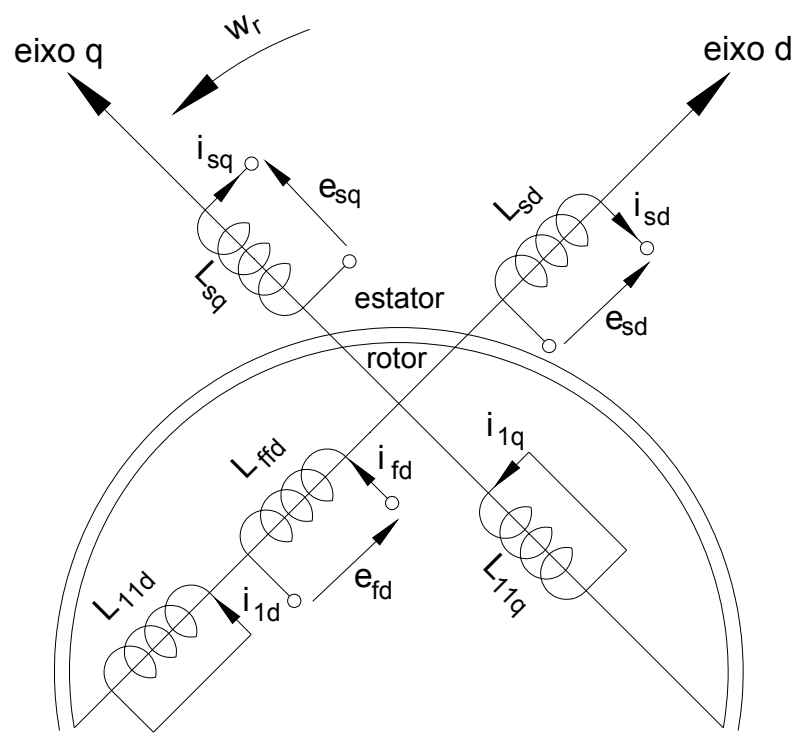

Figura 3.3 - Circuitos equivalentes do estator e rotor de um gerador síncrono

Os enrolamentos reais do estator são representados por duas bobinas fictícias dispostas sobre os eixos $d$ e $q$ com indutâncias próprias $L_{s d}$ e $L_{s q}$ respectivamente. Já o número de bobinas fictícias utilizadas para representar o rotor depende do tipo de construção do rotor (polos salientes ou cilíndrico) assim como da faixa de frequências para as quais o modelo deve representar com precisão a máquina. Para estudos de estabilidade dinâmica e transitória é prática comum representar o rotor de polos salientes por duas bobinas no eixo $d$, (correspondente ao enrolamento de campo e um enrolamento amortecedor com indutâncias próprias $L_{f f d}$ e $L_{11 d}$ 
respectivamente), e uma bobina no eixo $q$ (correspondente a um enrolamento amortecedor com indutância própria $L_{11 q}$ ). As indutâncias mútuas entre as bobinas mostradas na Figura 3.3, (em termos de quantidades físicas i.e., Henry), não são recíprocas e apresentam valores diferentes para cada par de bobinas acopladas. Entretanto, com a escolha de um adequado sistema em por unidade ${ }^{7}$ (pu), é possível transformar todas as indutâncias mútuas (sobre um mesmo eixo) em recíprocas e iguais entre si, independentemente do par de bobinas analisadas. Com esta simplificação, e desconsiderando os valores de sequência zero ${ }^{8}$, as equações em pu que representam o comportamento dinâmico do gerador síncrono se mostram a seguir, onde o ponto colocado sobre as variáveis denota a primeira derivada da variável com relação ao tempo e os subscritos $s d$, $s q, f d, 1 d, e 1 q$, são utilizados por conveniência para denotar grandezas relacionadas ao circuito de eixo direto de estator, eixo de quadratura do estator, circuito de campo, circuito amortecedor de eixo direto, e circuito amortecedor de eixo de quadratura respectivamente

Equações de tensão do estator:

$$
\begin{aligned}
& e_{s d}=\frac{1}{w_{b a s e}} \cdot \dot{\psi_{s d}}-\psi_{s q} \cdot w_{r}-R_{a} \cdot i_{s d} \\
& e_{s q}=\frac{1}{w_{\text {base }}} \cdot \dot{\psi_{s q}}+\psi_{s d} \cdot w_{r}-R_{a} \cdot i_{s q}
\end{aligned}
$$

Equações de tensão do rotor:

$$
e_{f d}=\frac{1}{w_{\text {base }}} \cdot \dot{\psi_{f d}}+R_{f d} \cdot i_{f d}
$$

\footnotetext{
${ }^{7}$ O sistema em pu adotado aqui é o Sistema Recíproco de Base $L_{a d}$. Ver referência [29] para maiores detalhes

${ }^{8}$ Geralmente para estudos de estabilidade dinâmica os circuitos são considerados equilibrados, o que faz a componente de sequência zero ser nula.
} 


$$
\begin{aligned}
& 0=\frac{1}{w_{\text {base }}} \cdot \dot{\psi_{1 d}}+R_{1 d} \cdot i_{1 d} \\
& 0=\frac{1}{w_{\text {base }}} \cdot \dot{\psi_{1 q}}+R_{1 q} \cdot i_{1 q}
\end{aligned}
$$

Equações de enlaces de fluxo do estator:

$$
\begin{gathered}
\psi_{s d}=-\left(L_{a d}+L_{l}\right) \cdot i_{s d}+L_{a d} \cdot i_{f d}+L_{a d} \cdot i_{1 d} \\
\psi_{s q}=-\left(L_{a q}+L_{l}\right) \cdot i_{s q}+L_{a q} \cdot i_{1 q}
\end{gathered}
$$

Equações de enlaces de fluxo do rotor:

$$
\begin{gathered}
\psi_{f d}=\left(L_{f d}+L_{a d}\right) \cdot i_{f d}+L_{a d} \cdot i_{1 d}-L_{a d} \cdot i_{s d} \\
\psi_{1 d}=L_{a d} \cdot i_{f d}+\left(L_{1 d}+L_{a d}\right) \cdot i_{1 d}-L_{a d} \cdot i_{s d} \\
\psi_{1 q}=\left(L_{1 q}+L_{a q}\right) \cdot i_{1 q}-L_{a q} \cdot i_{s q}
\end{gathered}
$$

Equação do torque:

$$
T_{e}=\psi_{s d} \cdot i_{s q}-\psi_{s q} \cdot i_{s d}
$$

Equações mecânicas

$$
\dot{w}_{r}=\frac{1}{2 H_{S}} \cdot\left(T_{m}-T_{e}-K_{S D} \cdot w_{r}\right)
$$




$$
\dot{\delta}=w_{\text {base }} \cdot\left(w_{r}-w_{\text {com }}\right)
$$

Onde,

$$
\begin{array}{ll}
e_{k}, i_{k}, \psi_{k} \quad= & \text { tensão, corrente e enlaces de fluxo do enrolamento " } k \text { " } \\
& (\text { com } k=s d, s q, f d, 1 d, 1 q) . \\
= & \text { resistência dos enrolamentos do rotor (com } k=f d, 1 d, 1 q) \\
R_{k} & =\text { resistência de armadura por fase. } \\
R_{a} & \text { indutância de dispersão do estator. } \\
L_{l} & =\text { indutância mútua estator-rotor de eixo direto e quadratura. } \\
L_{a d} \text { e } L_{a q} & =\text { indutância de dispersão do enrolamento de campo e do amortecedor } \\
L_{f d} \text { e } L_{1 d} & \text { de eixo direto. } \\
= & \text { indutância de dispersão do enrolamento amortecedor de eixo } \\
L_{1 q} & \text { de quadratura. } \\
= & \text { torque eletromagnético e torque mecânico. } \\
T_{e} e T_{m} & \text { coeficiente de atrito. } \\
K_{S D} & =\text { velocidade angular elétrica do rotor. } \\
w_{r} & =\text { velocidade angular elétrica da referência comum D-Q } \\
w_{c o m} & =\text { valor de base da velocidade angular elétrica [rad/s]. } \\
w_{b a s e} & \text { constante de inércia [s]. } \\
H_{S} & \text { posição angular do rotor com relação à referência D-Q [rad]. } \\
\delta &
\end{array}
$$

É importante mencionar que na análise dinâmica de grandes sistemas de potência é comum fazer duas simplificações às equações acima mostradas, com o intuito de diminuir o esforço computacional envolvido nas análises. A primeira consiste em desprezar os transitórios dos enrolamentos do estator $\left(\dot{\psi_{s d}} e \dot{\psi_{s q}}=0\right)$ que são de natureza eletromagnética e têm, em geral, constantes de tempo muito menores que aquelas associadas aos transitórios eletromecânicos. Sendo estes transitórios muito rápidos, pode-se considerar que o estator está operando em regime permanente senoidal, podendo se utilizar equações fasoriais (algébricas) para representar a rede, reduzindo assim a ordem dos modelos matemáticos e consequentemente 0 esforço computacional. A segunda simplificação consiste em desprezar o efeito de variação de velocidade do rotor sobre os termos $\psi_{s q}$. $w_{r}$ e $\psi_{s d}$. $w_{r}$ das equações de 
tensão do estator. Esta simplificação, em si, não contribui a diminuir o esforço computacional, porém compensa o efeito de desprezar os transitórios dos enrolamentos do estator [29] [30].

Considerando que as MGs são sistemas de potência relativamente pequenos não há motivos para realizar estas simplificações. Por outro lado, a interação dos rápidos inversores (desprovidos de inércia) das FEDs que não geram naturalmente em 60 $\mathrm{Hz}$ com os outros componentes da MG gera transitórios de alta frequência, existindo a necessidade de considerar os transitórios eletromagnéticos do estator da máquina síncrona, assim como o desenvolvimento de um modelo de rede compatível com as frequências destes transitórios [35] [36].

O modelo no espaço de estados desenvolvido a seguir considera as equações da máquina síncrona na sua forma original (sem simplificações) Para facilitar o desenvolvimento do modelo, nesta primeira fase se considera a máquina síncrona sem controle de excitação e velocidade, i.e., se considera a excitação do campo e o torque mecânico como constantes. A inclusão destes dois controles será discutida nas próximas seções.

As equações (3.1) à (3.5) junto com as equações (3.12) à (3.13) descrevem a dinâmica da máquina síncrona com $w_{r}, \delta, \psi_{s d}, \psi_{s q}, \psi_{f d}, \psi_{1 d}$ e $\psi_{1 q}$ como variáveis de estado. Porém, as derivadas destas variáveis aparecem nestas equações como função de $i_{s d}, i_{s q}, i_{f d}, i_{1 d}, i_{1 q} e T_{e}$, que não são nem variáveis de estado nem entradas. Consequentemente, é necessário expressar as correntes dos enrolamentos e o torque eletromagnético em termos das variáveis de estado. Das eq. (3.6) à (3.10) as correntes dos enrolamentos podem ser expressas em forma matricial como segue:

$$
\left[\begin{array}{l}
i_{s d} \\
i_{s q} \\
i_{f d} \\
i_{1 d} \\
i_{1 q}
\end{array}\right]=\left[\begin{array}{ccccc}
A_{S 11} & 0 & A_{S 13} & A_{S 14} & 0 \\
0 & A_{S 22} & 0 & 0 & A_{S 25} \\
A_{S 31} & 0 & A_{S 33} & A_{S 34} & 0 \\
A_{S 41} & 0 & A_{S 43} & A_{S 44} & 0 \\
0 & A_{S 52} & 0 & 0 & A_{S 55}
\end{array}\right] \cdot\left[\begin{array}{c}
\psi_{s d} \\
\psi_{s q} \\
\psi_{f d} \\
\psi_{1 d} \\
\psi_{1 q}
\end{array}\right]
$$

onde: 


$$
\begin{aligned}
& A_{S 11}=-\frac{1}{L_{l}+L_{a d} "} \\
& A_{S 13}=\frac{L_{a d} "}{L_{f d} \cdot\left(L_{l}+L_{a d} "\right)} \\
& A_{S 14}=\frac{L_{a d} "}{L_{1 d} \cdot\left(L_{l}+L_{a d}{ }^{\prime \prime}\right)} \\
& A_{S 22}=-\frac{1}{L_{l}+L_{a q} "} \\
& A_{S 25}=\frac{L_{a q} "}{L_{1 q} \cdot\left(L_{l}+L_{a q}{ }^{\prime}\right)} \\
& A_{S 31}=-\frac{m_{1}}{L_{l} \cdot\left(L_{f d}+m_{1}\right)} \\
& A_{S 33}=\frac{1}{L_{f d}+m_{1}} \\
& A_{S 34}=-\frac{m_{1}}{L_{1 d} \cdot\left(L_{f d}+m_{1}\right)} \\
& A_{S 41}=-\frac{m_{2}}{L_{l} \cdot\left(L_{1 d}+m_{2}\right)} \\
& A_{S 43}=-\frac{m_{2}}{L_{f d} \cdot\left(L_{1 d}+m_{2}\right)} \\
& A_{S 44}=\frac{1}{\left(L_{1 d}+m_{2}\right)} \\
& A_{S 52}=-\frac{n_{1}}{L_{l} .\left(L_{1 q}+n_{1}\right)} \\
& A_{S 55}=\frac{1}{L_{1 q}+n_{1}} \\
& m_{1}=\frac{1}{\frac{1}{L_{l}}+\frac{1}{L_{a d}}+\frac{1}{L_{1 d}}} \\
& L_{a d} "=\frac{1}{\frac{1}{L_{a d}}+\frac{1}{L_{f d}}+\frac{1}{L_{1 d}}} \\
& L_{a q} "=\frac{1}{\frac{1}{L_{a q}}+\frac{1}{L_{1 q}}} \\
& m_{2}=\frac{1}{\frac{1}{L_{l}}+\frac{1}{L_{a d}}+\frac{1}{L_{f d}}} \\
& n_{1}=\frac{1}{\frac{1}{L_{l}}+\frac{1}{L_{a q}}}
\end{aligned}
$$

Substituindo as expressões para as correntes da eq. (3.14) na eq.(3.11), e linearizando a expressão resultante por expansão em série de Taylor, permite obter a equação linearizada do torque eletromagnético em função unicamente das variáveis de estado.

$$
\Delta T_{e}=K_{S 1} \cdot \Delta \psi_{\mathrm{sd}}+K_{S 2} \cdot \Delta \psi_{\mathrm{sq}}+K_{S 3} \cdot \Delta \psi_{\mathrm{fd}}+K_{S 4} \cdot \Delta \psi_{1 \mathrm{~d}}+K_{S 5} \cdot \Delta \psi_{1 \mathrm{q}}
$$

onde: ${ }^{9}$

$$
\begin{gathered}
K_{S 1}=\frac{\psi_{s q 0}}{L_{a d} "+L_{l}}+\frac{-\psi_{s q 0}+\frac{L_{a q} " \cdot \psi_{1 q 0}}{L_{1 q}}}{L_{a q} "+L_{l}} \\
K_{S 2}=-\frac{\psi_{s d 0}}{L_{a q} "+L_{l}}-\frac{-\psi_{s d 0}+\frac{L_{a d} " \cdot \psi_{1 d 0}}{L_{1 d}}+\frac{L_{a d} " \cdot \psi_{f d 0}}{L_{f d}}}{L_{a d} "+L_{l}}
\end{gathered}
$$

${ }^{9} \mathrm{O}$ subscrito 0 identifica o valor da respectiva variável avaliado no ponto inicial de operação (valor pré-distúrbio). 


$$
\begin{aligned}
& K_{S 3}=-\frac{L_{a d} " \cdot \psi_{s q 0}}{L_{f d} \cdot\left(L_{l}+L_{a d} "\right)} \\
& K_{S 4}=-\frac{L_{a d} " \cdot \psi_{s q 0}}{L_{1 d} \cdot\left(L_{l}+L_{a d} "\right)} \\
& K_{S 5}=\frac{L_{a q} " \cdot \psi_{s d 0}}{L_{1 q} \cdot\left(L_{l}+L_{a q}{ }^{\prime \prime}\right)}
\end{aligned}
$$

Similarmente, substituindo as expressões para as correntes da eq. (3.14), nas eq. (3.1) à eq.(3.5), e linearizando a expressão resultante por expansão em série de Taylor, permite obter as equações de estado da máquina unicamente em função das variáveis de estado como se mostra abaixo:

$$
\begin{aligned}
{\left[\begin{array}{c}
\Delta \dot{w}_{r} \\
\Delta \dot{\delta} \\
\Delta \dot{\psi_{s d}} \\
\Delta \dot{\psi_{s q}} \\
\Delta \dot{\psi_{f d}} \\
\Delta \dot{\psi_{1 d}} \\
\Delta \dot{\psi_{1 q}}
\end{array}\right]=\left[\begin{array}{ccccccc}
a_{S 1,1} & 0 & a_{S 1,3} & a_{S 1,4} & a_{S 1,5} & a_{S 1,6} & a_{S 1,7} \\
a_{S 2,1} & 0 & 0 & 0 & 0 & 0 & 0 \\
a_{S 3,1} & 0 & a_{S 3,3} & a_{S 3,4} & a_{S 3,5} & a_{S 3,6} & 0 \\
a_{S 4,1} & 0 & a_{S 4,3} & a_{S 4,4} & 0 & 0 & a_{S 4,7} \\
0 & 0 & a_{S 5,3} & 0 & a_{S 5,5} & a_{S 5,6} & 0 \\
0 & 0 & a_{S 6,3} & 0 & a_{S 6,5} & a_{S 6,6} & 0 \\
0 & 0 & 0 & a_{S 7,4} & 0 & 0 & a_{S 7,7}
\end{array}\right] \cdot\left[\begin{array}{c}
\Delta w_{r} \\
\Delta \delta \\
\Delta \psi_{s d} \\
\Delta \psi_{s q} \\
\Delta \psi_{f d} \\
\Delta \psi_{1 d} \\
\Delta \psi_{1 q}
\end{array}\right] } \\
+\left[\begin{array}{cc}
0 & 0 \\
0 & 0 \\
w_{\text {base }} & 0 \\
0 & w_{\text {base }} \\
0 & 0 \\
0 & 0 \\
0 & 0
\end{array}\right]\left[\begin{array}{c}
0 \\
b 2_{S 2,1} \\
0 \\
0 \\
0 \\
0 \\
0
\end{array}\right] w_{c o m}+\left[\begin{array}{cc}
b e_{S 1,1} & 0 \\
0 & 0 \\
0 & 0 \\
0 & 0 \\
0 & w_{\text {base }} \\
0 & 0 \\
0 & 0
\end{array}\right]\left[\begin{array}{l}
\Delta T_{m} \\
\Delta e_{f d}
\end{array}\right]
\end{aligned}
$$

onde:

$$
\begin{array}{cccc}
a_{S 1,1}=-\frac{K_{S D}}{2 H_{S}} & a_{S 1,3}=-\frac{K_{S 1}}{2 H_{S}} & a_{S 1,4}=-\frac{K_{S 2}}{2 H_{S}} & a_{S 1,5}=-\frac{K_{S 3}}{2 H_{S}} \\
a_{S 1,6}=-\frac{K_{S 4}}{2 H_{S}} & a_{S 1,7}=-\frac{K_{S 5}}{2 H_{S}} & a_{S 2,1}=w_{\text {base }} & a_{S 3,1}=w_{\text {base }} \cdot \psi_{s q 0} \\
a_{S 3,3}=R_{a} \cdot w_{\text {base }} \cdot A_{S 1,1} & a_{S 3,4}=w_{r 0} \cdot w_{\text {base }} & a_{S 3,5}=R_{a} \cdot w_{\text {base }} \cdot \mathrm{A}_{\mathrm{S} 1,3} \\
a_{S 3,6}=R_{a} \cdot w_{\text {base }} \cdot \mathrm{A}_{\mathrm{S} 1,4} & a s_{4,1}=-w_{\text {base }} \cdot \psi_{s d 0} & a_{S 4,3}=-w_{\text {base }} \cdot w_{r 0} \\
a_{S 4,4}=R_{a} \cdot w_{\text {base }} \cdot \mathrm{A}_{\mathrm{S} 2,2} & a_{S 4,7}=R_{a} \cdot w_{\text {base }} \cdot \mathrm{A}_{\mathrm{S} 2,5} & a_{S 5,3}=-R_{f d} \cdot w_{\text {base }} \cdot \mathrm{A}_{\mathrm{S} 3,1}
\end{array}
$$




$$
\begin{array}{lrl}
a_{S 5,5}=-R_{f d} \cdot w_{\text {base }} \cdot A_{S 3,3} & a_{S 5,6}=-R_{f d} \cdot w_{\text {base }} \cdot A_{S 3,4} & a_{S 6,3}=-R_{1 d} \cdot w_{\text {base }} \cdot A_{S 4,1} \\
a_{S 6,5}=-R_{1 d} \cdot w_{\text {base }} \cdot A_{S 4,3} & a_{S 6,6}=-R_{1 d} \cdot w_{\text {base }} \cdot A_{S 4,4} & a_{S 7,4}=-R_{1 q} \cdot w_{\text {base }} \cdot A_{S 5,2} \\
a_{S 7,7}=-R_{1 q} \cdot w_{\text {base }} \cdot A_{S 5,5} & b 2_{S 2,1}=-w_{\text {base }} & b 3_{S 1,1}=\frac{1}{2 H_{S}}
\end{array}
$$

Até aqui se tem conseguido eliminar as correntes e o torque eletromagnético das equações de estado, no entanto, a eq.(3.16) ainda tem como entradas as tensões do estator $\left(\Delta e_{s d} e \Delta e_{s q}\right)$ na referência local $\mathrm{d}-\mathrm{q}$, sendo necessário expressá-las na referência comum D-Q. Para isto, utiliza-se a seguinte matriz de rotação:

$$
T=\left[\begin{array}{cc}
\cos (\delta) & -\operatorname{sen}(\delta) \\
\operatorname{sen}(\delta) & \cos (\delta)
\end{array}\right]
$$

A Figura 3.4 exemplifica o processo de transformação de coordenadas. Considere um vetor fictício girante " $V$ " decomposto em suas componentes direta $\left(V_{d}\right)$ e de quadratura $\left(V_{q}\right)$ sobre uma referência rotativa local d-q que gira com velocidade $w$. Este vetor pode ter suas componentes transladadas para uma referência rotativa global D-Q, que gira com velocidade $w_{c o m}$, através da seguinte expressão:

$$
\left[\begin{array}{l}
V_{D} \\
V_{Q}
\end{array}\right]=[T] \cdot\left[\begin{array}{l}
V_{d} \\
V_{q}
\end{array}\right]
$$

A eq. (3.18) é não linear e precisa ser linearizada. Aplicando a expansão em série de Taylor se tem: 


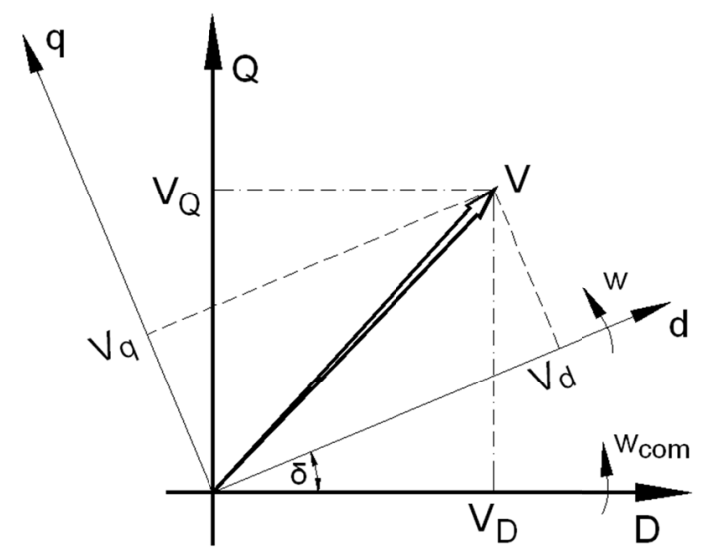

Figura 3.4 - Transformação de coordenadas d-q para D-Q

$$
\left[\begin{array}{l}
\Delta V_{D} \\
\Delta V_{Q}
\end{array}\right]=\left[T_{s}\right] \cdot\left[\begin{array}{l}
\Delta V_{d} \\
\Delta V_{q}
\end{array}\right]+\left[T_{c}\right] \cdot \Delta \delta
$$

onde

$$
T_{s}=\left[\begin{array}{cc}
\cos \left(\delta_{0}\right) & -\operatorname{sen}\left(\delta_{0}\right) \\
\operatorname{sen}\left(\delta_{0}\right) & \cos \left(\delta_{0}\right)
\end{array}\right] \quad T_{c}=\left[\begin{array}{c}
-V_{d 0} \cdot \operatorname{sen}\left(\delta_{0}\right)-V_{q 0} \cdot \cos \left(\delta_{0}\right) \\
V_{d 0} \cdot \cos \left(\delta_{0}\right)-V_{q 0} \cdot \operatorname{sen}\left(\delta_{0}\right)
\end{array}\right]
$$

A transformação inversa, i.e., transladar as componentes de um vetor da referência D-Q para a referência d-q, se obtém resolvendo a eq.(3.19) para as componentes $V_{d}$ e $V_{q}$ e simplificando a expressão resultante:

$$
\left[\begin{array}{l}
\Delta V_{d} \\
\Delta V_{q}
\end{array}\right]=\left[T_{s}\right]^{-1} \cdot\left[\begin{array}{l}
\Delta V_{D} \\
\Delta V_{Q}
\end{array}\right]+\left[T_{v}\right] \cdot \Delta \delta
$$

Onde

$$
\left[T_{S}\right]^{-1}=\left[\begin{array}{cc}
\cos \left(\delta_{0}\right) & \operatorname{sen}\left(\delta_{0}\right) \\
-\operatorname{sen}\left(\delta_{0}\right) & \cos \left(\delta_{0}\right)
\end{array}\right] \quad T_{v}=\left[\begin{array}{l}
-V_{D 0} \cdot \operatorname{sen}\left(\delta_{0}\right)+V_{Q 0} \cdot \cos \left(\delta_{0}\right) \\
-V_{D 0} \cdot \cos \left(\delta_{0}\right)-V_{Q 0} \cdot \operatorname{sen}\left(\delta_{0}\right)
\end{array}\right]
$$


Ao aplicar a eq. (3.20) as componentes $\Delta e_{s d} e \Delta e_{s q}$ da eq. (3.16), se tem as tensões nos terminais do gerador transladadas para a referência comum $\left(v_{b D Q}\right)$, obtendo-se assim, o modelo da máquina síncrona na forma desejada, com $\Delta T_{m} e \Delta e_{f d}$ constantes, como mostra a equação abaixo.

$$
\begin{aligned}
& {\left[\begin{array}{c}
\Delta \dot{w_{r}} \\
\Delta \dot{\delta} \\
\Delta \dot{\psi_{s d}} \\
\Delta \dot{\psi_{s q}} \\
\Delta \dot{\psi_{f d}} \\
\Delta \dot{\psi_{1 d}} \\
\Delta \dot{\psi_{1 q}}
\end{array}\right]=\left[\begin{array}{ccccccc}
a_{S 1,1} & 0 & a_{S 1,3} & a_{S 1,4} & a_{S 1,5} & a_{S 1,6} & a_{S 1,7} \\
a_{S 2,1} & 0 & 0 & 0 & 0 & 0 & 0 \\
a_{S 3,1} & a_{S 3,2} & a_{S 3,3} & a_{S 3,4} & a_{S 3,5} & a_{S 3,6} & 0 \\
a_{S 4,1} & a_{S 4,2} & a_{S 4,3} & a_{S 4,4} & 0 & 0 & a_{S 4,7} \\
0 & 0 & a_{S 5,3} & 0 & a_{S 5,5} & a_{S 5,6} & 0 \\
0 & 0 & a_{S 6,3} & 0 & a_{S 6,5} & a_{S 6,6} & 0 \\
0 & 0 & 0 & a_{S 7,4} & 0 & 0 & a_{S 7,7}
\end{array}\right] \cdot\left[\begin{array}{c}
\Delta w_{r} \\
\Delta \delta \\
\Delta \psi_{s d} \\
\Delta \psi_{s q} \\
\Delta \psi_{f d} \\
\Delta \psi_{1 d} \\
\Delta \psi_{1 q}
\end{array}\right]} \\
& +\left[\begin{array}{cc}
0 & 0 \\
0 & 0 \\
b 1_{S 3,1} & b 1_{S 3,2} \\
b 1_{S 4,1} & b 1_{S 4,2} \\
0 & 0 \\
0 & 0 \\
0 & 0
\end{array}\right]\left[\begin{array}{c}
\Delta v_{b D} \\
\Delta v_{b Q}
\end{array}\right]+\left[\begin{array}{c}
0 \\
b 2_{S 2,1} \\
0 \\
0 \\
0 \\
0 \\
0
\end{array}\right] \Delta w_{c o m}+\left[\begin{array}{cc}
b 3_{S 1,1} & 0 \\
0 & 0 \\
0 & 0 \\
0 & 0 \\
0 & w_{\text {base }} \\
0 & 0 \\
0 & 0
\end{array}\right]\left[\begin{array}{c}
\Delta T_{m} \\
\Delta e_{f d}
\end{array}\right]
\end{aligned}
$$

onde:

$$
\begin{aligned}
& a_{S 3,2}=w_{b a s e} \cdot\left[-v_{b D 0} \cdot \operatorname{sen}\left(\delta_{0}\right)+v_{b Q 0} \cdot \cos \left(\delta_{0}\right)\right] \\
& a_{S 4,2}=w_{b a s e} \cdot\left[-v_{b D 0} \cdot \cos \left(\delta_{0}\right)-v_{b Q 0} \cdot \operatorname{sen}\left(\delta_{0}\right)\right] \\
& b 1_{S 3,1}=w_{\text {base }} \cdot \cos \left(\delta_{0}\right) \\
& b 1_{S 3,2}=w_{\text {base }} \cdot \operatorname{sen}\left(\delta_{0}\right) \\
& b 1_{S 4,1}=-w_{\text {base }} \cdot \operatorname{sen}\left(\delta_{0}\right) \\
& b 1_{S 4,2}=w_{\text {base }} \cdot \cos \left(\delta_{0}\right)
\end{aligned}
$$

\subsubsection{Modelagem do Sistema de Excitação}

Existem diversos tipos de sistemas de excitação, tanto no que se refere ao número de componentes, quanto ao princípio básico de funcionamento. De uma forma geral, os sistemas de excitação podem ser classificados em sistema de excitação rotativos e sistema de excitação estático. Um sistema do tipo rotativo considera a presença de excitatriz, sendo esta um gerador de corrente contínua, uma máquina de 
relutância ou um alternador. Já um sistema de excitação do tipo estático considera a utilização de tiristores estáticos controlados, alimentados pelos próprios terminais do gerador ou pelo barramento auxiliar da usina, dispensando as unidades rotativas.

GGDs de pequeno porte (até $250 \mathrm{~kW}$ ) são normalmente equipados com sistemas de excitação estáticos com controle implementado digitalmente, onde a função básica do sistema é suprir e, automaticamente ajustar a corrente do enrolamento de campo de forma a manter constante a tensão de saída da máquina síncrona face às variações de carga. Sistemas Estabilizadores de Potência (PSS) geralmente não são encontrados no sistema de excitação destes grupos geradores. [37] [38] [39] [40].

$\mathrm{Na}$ referência [41] são definidos pelo IEEE modelos genéricos dos sistemas de excitação mais utilizados nos estudos de elétricos de potência. Tais modelos têm o propósito de servir como diretrizes para os estudos de estabilidade dinâmica e transitória e refletem o comportamento real do sistema da melhor maneira possível. Para representar o sistema de excitação de geradores diesel de pequeno porte, neste trabalho faz-se uso do modelo genérico IEEE tipo ST6B, por ser este o mais adequado para representar sistemas de excitação implementados digitalmente. $\mathrm{O}$ diagrama de blocos se mostra na Figura 3.5. Além de regular a tensão do gerador através de uma malha de controle externa baseada num controle proporcionalintegral (PI), o sistema de excitação ST6B inclui um circuito limitador da corrente de campo e uma malha de controle interna estabilizadora, projetada para aumentar a resposta dinâmica do sistema assim como o desempenho em regime.

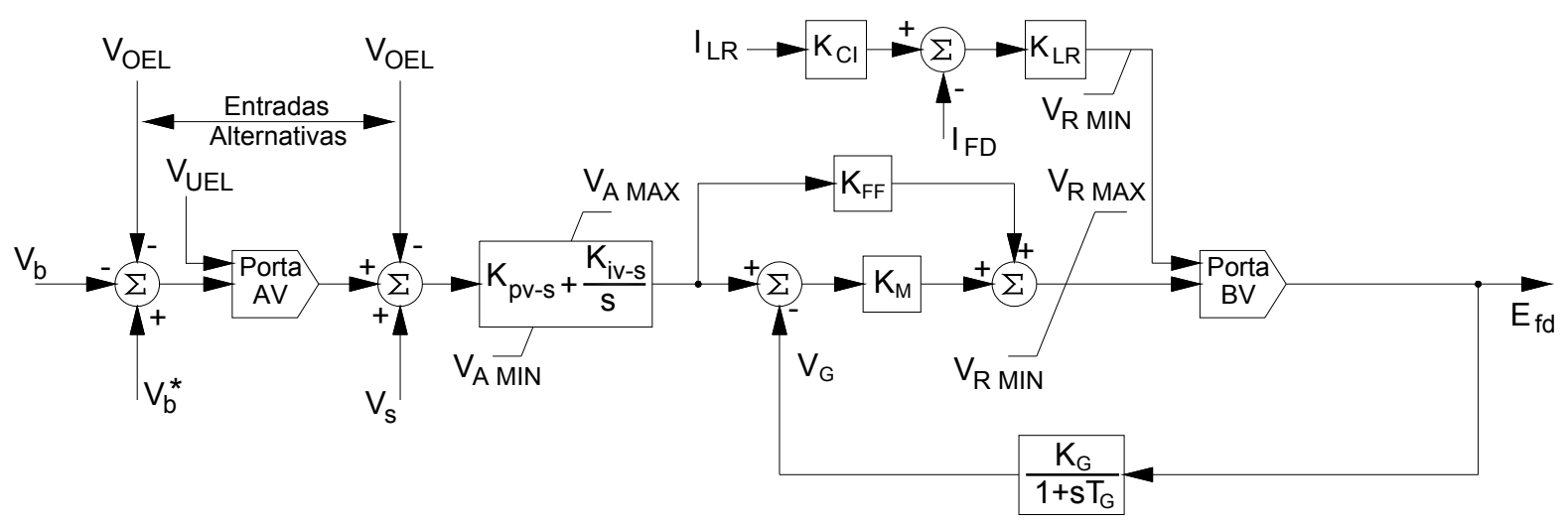

Figura 3.5 - Sistema de excitação estático IEEE - Tipo ST6B 
Na Figura 3.5, $v_{b}$ é a medição da tensão nos terminais do gerador e $v_{b}{ }^{*}$ é a tensão de referência ${ }^{10}$. A diferença entre essas duas grandezas constitui o sinal de erro básico. São feitas provisões para sinais de erros adicionais: $V_{s}$ é o sinal de erro de um PSS, e $V_{U E L}$ e $V_{O E L}$ são os sinais de erro dos limitadores de subexcitação e sobreexcitação respectivamente. O limite da corrente de campo é definido pelo parâmetro $I_{L R}$, valor que é comparado com o valor da corrente de campo medida $I_{F D}$ para gerar o sinal de erro. $K_{p v-S}$ e $K_{i v-S}$ constituem os ganhos proporcional e integral respectivamente do PI da malha de controle externa da tensão, enquanto $K_{M}$ e $K_{F F}$ representam os ganhos feedforward e pré-feedforward respectivamente da malha interna estabilizadora. Já $K_{G}$ e $T_{G}$ representam o ganho e a constante de tempo da realimentação (feedback) da malha interna estabilizadora. Portas AV (High Value Gate) e Portas BV (Low Value Gate) são utilizadas em alguns pontos do diagrama de blocos, para selecionar o sinal de entrada que tem o nível mais alto ou mais baixo respectivamente. A saída do sistema de excitação $E_{f d}$ corresponde à tensão aplicada ao enrolamento de campo expressa num sistema em pu conhecido como sistema por unidade não recíproco, amplamente utilizado pelos fabricantes de sistemas de excitação e diferente do utilizado neste trabalho para modelar o gerador síncrono. Consequentemente, uma conversão de bases é necessária para a interface dos dois modelos. Por definição $E_{f d}=\frac{L_{a d u}}{R_{f d}} \cdot e_{f d}$, [29] onde $L_{a d u}$ corresponde ao valor não saturado de $L_{a d}$.

Algumas simplificações podem ser feitas ao sistema de excitação ST6B no estudo da estabilidade dinâmica do GGD. As não linearidades associadas ao circuito limitador da corrente de campo podem ser ignoradas, devido ao fato de se estar interessado em desenvolver um modelo linear ao redor de um ponto de operação tal que $E_{f d}$ esteja dentro dos seus limites operativos. O mesmo se aplica aos limitadores de subexcitação e sobreexcitação, assim como aos limitadores do controle PI $\left(V_{A \text { MAX }}\right.$ e $\left.V_{A M I N}\right)$. Já o sinal de erro $V_{S}$ pode ser suprimido com base no fato de GGDs de pequeno porte não serem geralmente equipados com sistemas

\footnotetext{
${ }^{10}$ No que se segue deste trabalho, o asterisco sobrescrito $\left({ }^{*}\right)$ denota o valor de referência (valor alvo) da respectiva variável.
} 
PSS. O diagrama de blocos do sistema de excitação assim simplificado se mostra na Figura 3.6.

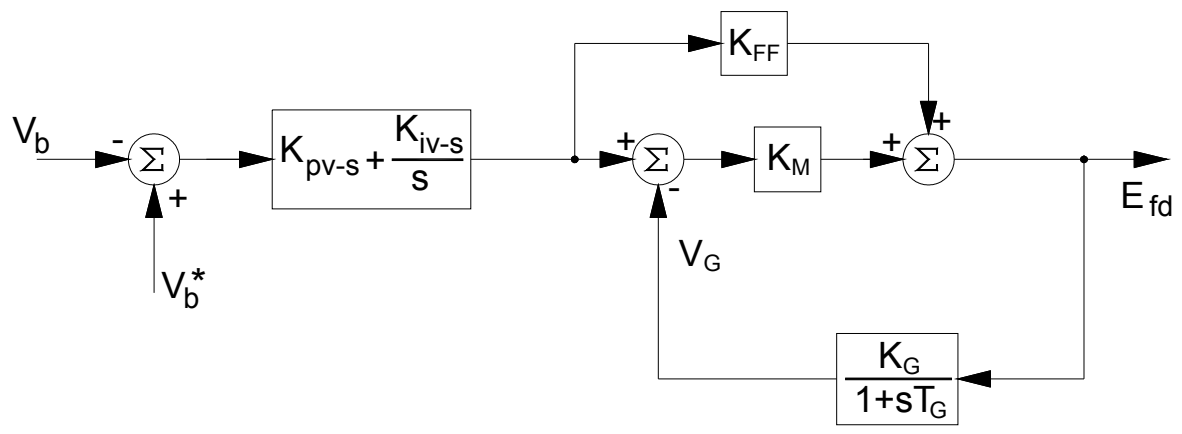

Figura 3.6 - Sistema de excitação estático ST6B - Simplificado

O diagrama de blocos da Figura 3.6 pode ser manipulado de forma a facilitar o desenvolvimento do modelo no espaço de estados. Com as apropriadas transformações, a malha interna de controle pode ser reduzida a dois blocos como se mostra na Figura 3.7.

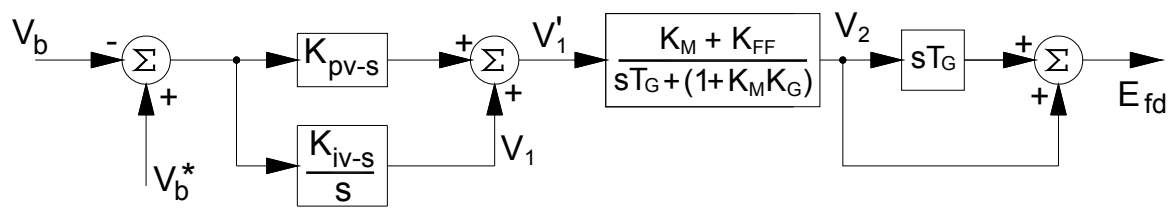

Figura 3.7 - Diagrama de blocos reduzido - Sistema ST6B simplificado

Da Figura 3.7, as equações que descrevem o comportamento da malha externa de controle são:

$$
\begin{gathered}
\dot{v}_{1}=K_{i v-s \cdot}\left(v_{b}{ }^{*}-v_{b}\right) \\
v_{1}{ }^{\prime}=v_{1}+K_{p v-s} \cdot\left(v_{b}{ }^{*}-v_{b}\right)
\end{gathered}
$$

Linearizando eq.(3.22): 


$$
\Delta \dot{v}_{1}=-K_{i v-S} \cdot \Delta v_{b}
$$

O termo $v_{b}$ é o módulo da tensão nos terminais do gerador, que pode ser calculado em termos das componentes d-q como segue:

$$
v_{b}=\sqrt{e_{s d}^{2}+e_{s q}^{2}}
$$

Linearizando a eq. (3.25) por expansão em séries de Taylor, e substituindo na eq.(3.24):

$$
\Delta \dot{v}_{1}=-\frac{K_{i v-S}}{v_{b 0}} \cdot\left(e_{s d 0} \cdot \Delta e_{s d}+e_{s q 0} \cdot \Delta e_{s q}\right)
$$

Sendo,

$$
v_{b 0}=\sqrt{e_{s d 0}^{2}+e_{s q 0}^{2}}
$$

Aplicando a eq. (3.20) as componentes $\Delta e_{s d} e \Delta e_{s q}$ da eq.(3.26) e simplificando se tem:

$$
\Delta \dot{v}_{1}=a_{S 8,2} \cdot \Delta \delta+b 1_{S 8,1} \cdot \Delta v_{b D}+b 1_{S 8,2} \cdot \Delta v_{b Q}
$$

Onde,

$$
\begin{gathered}
a_{S 8,2}=-\frac{K_{i v-S}}{v_{b 0}}\left[e _ { s d 0 } \left(-v_{b D 0} \operatorname{sen}\left(\delta_{0}\right)+v_{b Q 0} \cos \left(\delta_{0}\right)+e_{s q 0}\left(-v_{b D 0} \cos \left(\delta_{0}\right)-v_{b Q 0} \cdot \operatorname{sen}\left(\delta_{0}\right)\right]\right.\right. \\
b 1_{S 8,1}=-\frac{K_{i v-S}}{v_{b 0}}\left[e_{s d 0} \cdot \cos \left(\delta_{0}\right)-e_{s q 0} \cdot \operatorname{sen}\left(\delta_{0}\right)\right.
\end{gathered}
$$




$$
b 1_{S 8,2}=-\frac{K_{i v-S}}{v_{b 0}}\left[e_{s d 0} \cdot \operatorname{sen}\left(\delta_{0}\right)+e_{s q 0} \cdot \cos \left(\delta_{0}\right)\right.
$$

De forma similar, substituindo a eq. (3.25) na eq. (3.23) e linearizando a expressão resultante se tem:

$$
\Delta v_{1}{ }^{\prime}=A_{S 8,2} \cdot \Delta \delta+B 1_{S 8,1} \cdot \Delta v_{b D}+B 1_{S 8,2} \cdot \Delta v_{b Q}+\Delta v_{1}
$$

Onde,

$$
\begin{gathered}
A_{S 8,2}=\frac{K_{p v-S}}{-v_{b 0}}\left[e_{s d 0}\left(-v_{b D 0} \operatorname{sen}\left(\delta_{0}\right)+v_{b Q 0} \cos \left(\delta_{0}\right)\right)+e_{s q 0}\left(-v_{b D 0} \cos \left(\delta_{0}\right)-v_{b Q 0} \operatorname{sen}\left(\delta_{0}\right)\right)\right] \\
B 1_{S 8,1}=-\frac{K_{p v-S}}{v_{b 0}}\left[e_{s d 0} \cdot \cos \left(\delta_{0}\right)-e_{s q 0} \cdot \operatorname{sen}\left(\delta_{0}\right)\right] \\
B 1_{S 8,2}=-\frac{K_{p v-S}}{v_{b 0}}\left[e_{s d 0} \cdot \operatorname{sen}\left(\delta_{0}\right)+e_{s q 0} \cdot \cos \left(\delta_{0}\right)\right]
\end{gathered}
$$

Voltando novamente para a Figura 3.7, as equações que descrevem o comportamento da malha interna de controle são:

$$
\begin{gathered}
\dot{v}_{2}=\frac{K_{F F}+K_{M}}{T_{G}} \cdot v_{1}{ }^{\prime}-\frac{1+K_{M} \cdot K_{G}}{T_{G}} \cdot v_{2} \\
E_{f d}=T_{G} \cdot \dot{v}_{2}+v_{2}
\end{gathered}
$$

Linearizando a eq. (3.29) e substituindo a eq. (3.28) na expressão resultante se tem: 


$$
\Delta \dot{v}_{2}=a_{S 9,2} \cdot \Delta \delta+a_{S 9,8} \cdot \Delta v_{1}+a_{S 9,9} \cdot \Delta v_{2}+b 1_{S 9,1} \Delta v_{b D}+b 1_{S 9,2} \Delta v_{b Q}
$$

Sendo,

$$
\begin{aligned}
& a_{S 9,2}=A_{S 8,2} \cdot \frac{K_{F F}+K_{M}}{T_{G}} \quad a_{S 9,8}=\frac{K_{F F}+K_{M}}{T_{G}} \quad a_{S 9,9}=-\frac{1+K_{M} \cdot K_{G}}{T_{G}} \\
& b 1_{S 9,1}=B 1_{S 8,1} \cdot \frac{K_{F F}+K_{M}}{T_{G}} \quad b 1_{S 9,2}=B 1_{S 8,2} \cdot \frac{K_{F F}+K_{M}}{T_{G}}
\end{aligned}
$$

De maneira similar, linearizando eq. (3.30) e substituindo a eq. (3.31) na expressão resultante se tem:

$$
\Delta E_{f d}=A A_{S 5,2} \cdot \Delta \delta+A A_{S 5,8} \cdot \Delta v_{1}+A A_{S 5,9} \Delta v_{2}+B 1_{S 5,1} \cdot \Delta v_{b D}+B 1_{S 5,2} \cdot \Delta v_{b Q}
$$

Onde,

$$
\begin{array}{lll}
A A_{S 5,2}=T_{G} \cdot a_{S 9,2} & A A_{S 5,8}=T_{G} \cdot a_{S 9,8} & A A_{S 5,9}=-K_{M} \cdot K_{G} \\
B 1_{S 5,1}=T_{G} \cdot b 1_{S 9,1} & B 1_{S 5,2}=T_{G} \cdot b 1_{S 9,2} &
\end{array}
$$

Equações (3.27), (3.31) e (3.32) descrevem a dinâmica do sistema de excitação STB6 simplificado.

\subsubsection{Modelagem do Motor Diesel e do Regulador de Velocidade}

O motor diesel, de um GGD destinado ao suprimento de energia elétrica, é formado pelo motor diesel propriamente dito e por um conjunto de dispositivos destinados ao controle da velocidade (rotação), conhecidos como reguladores ou governadores. Existem dois tipos básicos de governadores, que são: mecânicos e eletrônicos. Grupos geradores diesel de pequeno porte são normalmente equipados com reguladores eletrônicos [38] [39] [42]. 
Os reguladores eletrônicos são constituídos por três elementos básicos: sensor de rotação, regulador eletrônico propriamente dito e o atuador. A construção pode variar conforme o fabricante, mas todos funcionam segundo os mesmos princípios. O sensor de rotação induz um pulso de corrente elétrica que é captado pelo regulador. A quantidade de pulsos por segundo (frequência) é comparada, pelo regulador, com o valor de referência. Se houver diferença, o regulador altera o fluxo de corrente enviada para o atuador que efetua as correções no suprimento de combustível para mais ou para menos, conforme necessidade.

Um modelo dinâmico que representa satisfatoriamente um motor diesel de pequeno porte equipado com regulador eletrônico se mostra na Figura 3.8 [43] [44] [45]. Devido às pequenas constantes de tempo associadas ao sensor de rotação, quando comparadas com os outros elementos do sistema, se considera sua modelagem desnecessária.

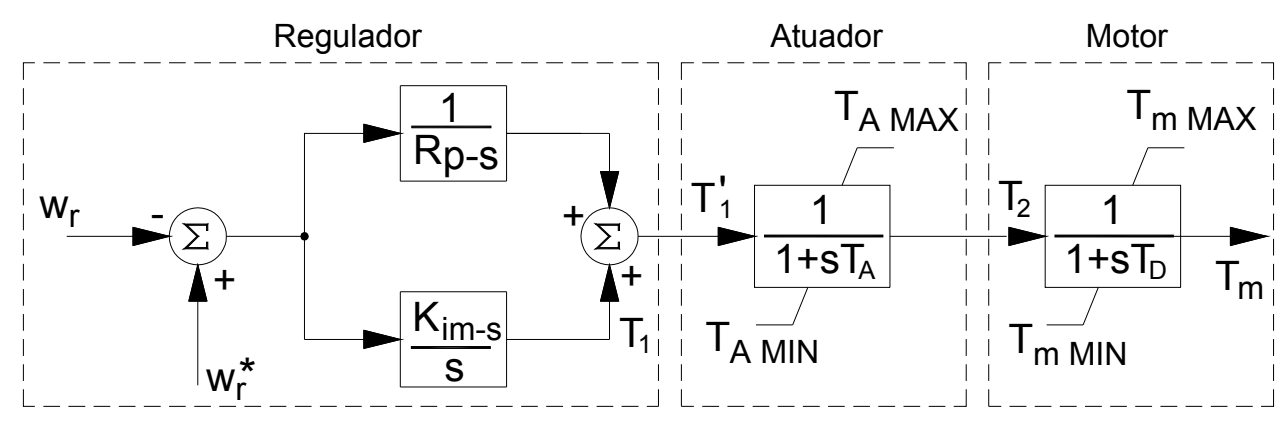

Figura 3.8 - Diagrama de blocos do motor diesel com regulador

Na Figura 3.8, $w_{r}$ é a velocidade de rotação do motor, e $R_{p-s}$ é o droop de velocidade (variação em pu entre as velocidades nominais em vazio e a final, com aplicação de $100 \%$ de carga). Já $K_{i m-S}$ é o ganho integral da malha de controle do regulador e desempenha o papel do controle secundário dos grandes sistemas de potência, i.e., restabelecer lentamente a frequência do sistema ao seu valor programado. As constantes $T_{A}$ e $T_{D}$ são as constantes de tempo associadas ao atuador e ao motor respectivamente e $T_{m}$ é o torque mecânico de saída aplicado ao eixo do gerador síncrono. Todas as variáveis estão expressas em pu na base dos valores nominais do gerador síncrono. 
Da Figura 3.8, as equações que descrevem o comportamento do regulador de velocidade são:

$$
\begin{gathered}
\dot{T}_{1}=K_{i m-s} \cdot\left(w_{r}{ }^{*}-w_{r}\right) \\
T_{1}{ }^{\prime}=T_{1}+\frac{1}{R_{p-S}} \cdot\left(w_{r}{ }^{*}-w_{r}\right)
\end{gathered}
$$

Linearizando a eq.(3.33) e eq. (3.34) se tem:

$$
\begin{gathered}
\Delta \dot{T}_{1}=-K_{\text {im-s }} \cdot \Delta w_{r} \\
\Delta T_{1}{ }^{\prime}=\Delta T_{1}-\frac{1}{R_{p-S}} \cdot \Delta w_{r}
\end{gathered}
$$

Voltando para Figura 3.8, as equações que descrevem o comportamento do atuador e do motor diesel são:

$$
\begin{aligned}
& \dot{T_{2}}=\frac{1}{T_{A}} \cdot\left(T_{1}{ }^{\prime}-T_{2}\right) \\
& \dot{T_{m}}=\frac{1}{T_{D}} \cdot\left(T_{2}-T_{m}\right)
\end{aligned}
$$


Substituindo a eq. (3.34) na eq. (3.37) e linearizando a expressão resultante se tem:

$$
\Delta \dot{\mathrm{T}}_{2}=\frac{1}{\mathrm{~T}_{\mathrm{A}}} \cdot\left(-\frac{1}{R_{p-S}} \cdot \Delta w_{r}+\Delta T_{1}-\Delta T_{2}\right)
$$

Linearizando a eq. (3.38):

$$
\Delta T_{m}=\frac{1}{T_{D}} \cdot\left(\Delta T_{2}-\Delta T_{m}\right)
$$

Equações (3.35) (3.39) e (3.40) descrevem a dinâmica do motor diesel e do regulador de velocidade.

\subsubsection{Modelo completo do grupo gerador diesel}

O modelo completo do GGD no espaço de estados se obtém ao combinar adequadamente as EDOs desenvolvidas individualmente para o gerador, o sistema de excitação, o regulador de velocidade e o motor diesel e se mostra a seguir:

$$
\left[\Delta \dot{X}_{S I N}\right]=\left[A_{S I N}\right] \cdot\left[\Delta X_{S I N}\right]+\left[B 1_{S I N}\right] \cdot\left[\Delta v_{b D Q}\right]+\left[B 2_{S I N}\right] \cdot \Delta w_{c o m}
$$

Onde, 


$$
\begin{aligned}
& {\left[A_{S I N}\right]=\left[\begin{array}{cccccccccccc}
a_{S 1,1} & 0 & a_{S 1,3} & a_{S 1,4} & a_{S 1,5} & a_{S 1,6} & a_{S 1,7} & 0 & 0 & 0 & 0 & a_{S 1,12} \\
a_{S 2,1} & 0 & 0 & 0 & 0 & 0 & 0 & 0 & 0 & 0 & 0 & 0 \\
a_{S 3,1} & a_{S 3,2} & a_{S 3,3} & a_{S 3,4} & a_{S 3,5} & a_{S 3,6} & 0 & 0 & 0 & 0 & 0 & 0 \\
a_{S 4,1} & a_{S 4,2} & a_{S 4,3} & a_{S 4,4} & 0 & 0 & a_{S 4,7} & 0 & 0 & 0 & 0 & 0 \\
0 & a_{S 5,2} & a_{S 5,3} & 0 & a_{S 5,5} & a_{S 5,6} & 0 & a_{S 5,8} & a_{S 5,9} & 0 & 0 & 0 \\
0 & 0 & a_{S 6,3} & 0 & a_{S 6,5} & a_{S 6,6} & 0 & 0 & 0 & 0 & 0 & 0 \\
0 & 0 & 0 & a_{S 7,4} & 0 & 0 & a_{S 7,7} & 0 & 0 & 0 & 0 & 0 \\
0 & a_{S 8,2} & 0 & 0 & 0 & 0 & 0 & 0 & 0 & 0 & 0 & 0 \\
0 & a_{S 9,2} & 0 & 0 & 0 & 0 & 0 & a_{S 9,8} & a_{S 9,9} & 0 & 0 & 0 \\
a_{S 10,1} & 0 & 0 & 0 & 0 & 0 & 0 & 0 & 0 & 0 & 0 & 0 \\
a_{S 11,1} & 0 & 0 & 0 & 0 & 0 & 0 & 0 & 0 & a_{S 11,10} & a_{S 11,11} & 0 \\
0 & 0 & 0 & 0 & 0 & 0 & 0 & 0 & 0 & 0 & a_{S 12,11} & a_{S 12,12}
\end{array}\right]} \\
& {\left[\Delta X_{S I N}\right]=\left[\begin{array}{c}
\Delta w_{r} \\
\Delta \delta \\
\Delta \psi_{\mathrm{sd}} \\
\Delta \psi_{\mathrm{sq}} \\
\Delta \psi_{\mathrm{fd}} \\
\Delta \psi_{1 \mathrm{~d}} \\
\Delta \psi_{1 \mathrm{q}} \\
\Delta v_{1} \\
\Delta v_{2} \\
\Delta T_{1} \\
\Delta T_{2} \\
\Delta T_{m}
\end{array}\right]} \\
& {\left[B 1_{S I N}\right]=\left[\begin{array}{cc}
0 & 0 \\
0 & 0 \\
b 1_{S 3,1} & b 1_{S 3,2} \\
b 1_{S 4,1} & b 1_{S 4,2} \\
b 1_{S 5,1} & b 1_{S 5,2} \\
0 & 0 \\
0 & 0 \\
b 1_{S 8,1} & b 1_{S 8,2} \\
b 1_{S 9,1} & b 1_{S 9,2} \\
0 & 0 \\
0 & 0 \\
0 & 0
\end{array}\right]} \\
& {\left[B 2_{S I N}\right]=\left[\begin{array}{c}
0 \\
b 2_{S 2,1} \\
0 \\
0 \\
0 \\
0 \\
0 \\
0 \\
0 \\
0 \\
0 \\
0
\end{array}\right]}
\end{aligned}
$$




$$
\begin{aligned}
& a_{S 1,12}=\frac{1}{2 H_{S}} \\
& a_{S 5,2}=A A_{S 5,2} \cdot b 3_{S 5,1} \\
& a_{S 5,8}=A A_{S 5,8} \cdot b 3_{S 5,1} \\
& a_{S 5,9}=A A_{S 5,9} \cdot b 3_{S 5,1} \\
& a_{S 10,1}=-K_{i m-S} \\
& a_{S 11,10}=\frac{1}{T_{A}} \\
& a_{S 11,11}=-\frac{1}{T_{A}} \\
& a_{S 12,12}=-\frac{1}{T_{D}} \\
& b 1_{S 5,1}=B 1_{S 5,1} \cdot b 3_{S 5,1} \\
& a_{S 11,1}=-\frac{1}{R_{p-S} \cdot T_{A}} \\
& a_{S 12,11}=\frac{1}{T_{D}} \\
& {\left[v_{b D Q}\right]=\left[\begin{array}{l}
v_{b D} \\
v_{b Q}
\end{array}\right]} \\
& b 1_{S 5,2}=B 1_{S 5,2} \cdot b 3_{S 5,1} \\
& b 3_{S 5,1}=R_{f d} \cdot \frac{w_{\text {base }}}{L_{a d u}}
\end{aligned}
$$

As equações relacionando as saídas do modelo (i.e., as correntes do estator na referência comum D-Q) com as variáveis de estado, podem ser obtidas a partir da linearização das primeiras duas filas da eq. (3.14) em combinação com a eq. (3.19):

$$
\left[\Delta i_{S D Q}\right]=\left[C 1_{S I N}\right] \cdot\left[\Delta X_{S I N}\right]
$$

Onde

$$
\begin{gathered}
C 1_{S I N}=\left[T_{S}\right] \cdot\left[Z_{1}\right]+\left[T_{c}\right] \cdot\left[Z_{2}\right] \\
{\left[Z_{1}\right]=\left[\begin{array}{cccccccccccc}
0 & 0 & c 1_{S 1,3} & 0 & c 1_{S 1,5} & c 1_{S 1,6} & 0 & 0 & 0 & 0 & 0 & 0 \\
0 & 0 & 0 & c 1_{S 2,4} & 0 & 0 & c 1_{S 2,7} & 0 & 0 & 0 & 0 & 0
\end{array}\right]} \\
{\left[z_{2}\right]=\left[\begin{array}{llllllllllll}
0 & 1 & 0 & 0 & 0 & 0 & 0 & 0 & 0 & 0 & 0 & 0
\end{array}\right]} \\
c 1_{S 2,4}=-\frac{1}{L_{l}+L_{a d} "} \\
c 1_{S 1,5}=\frac{L_{a d}}{L_{l}+L_{a q} "} \\
c 1_{S 2,7}=\frac{L_{a q}{ }^{\prime \prime}}{L_{1 q} \cdot\left(L_{l}+L_{a d}{ }^{\prime \prime}\right)}
\end{gathered}
$$


No caso da referência local $d-q$ do grupo gerador diesel ser escolhida como a referência comum D-Q do sistema, é necessário também expressar a velocidade em função das variáveis de estado através da seguinte expressão:

$$
\Delta w_{\text {com }}=\left[C 2_{S I N}\right] \cdot\left[\Delta X_{S I N}\right]
$$

Sendo,

$$
C 2_{S I N}=\left[\begin{array}{llllllllllll}
1 & 0 & 0 & 0 & 0 & 0 & 0 & 0 & 0 & 0 & 0 & 0
\end{array}\right]
$$

\subsection{Modelagem dos Inversores}

As técnicas de controle mais comumente utilizadas em inversores que servem de interface para as FEDs que não geram naturalmente em 50 ou $60 \mathrm{~Hz}$ com a rede foram brevemente mencionadas no Capítulo 1. Nesta seção se abordará em detalhe estas técnicas de controle e sua modelagem no espaço de estados. Entretanto, antes de entrar em detalhes é importante fazer algumas considerações relacionadas às funções que cada microgerador desempenha dentro de uma MG isolada. Nesta perspectiva, as FEDs podem ser caracterizadas de duas formas distintas:

- FEDs formadoras de rede - definem a referência de tensão e frequência de modo a assumir uma resposta rápida aos desequilíbrios entre geração e carga. Geralmente estão associadas às fontes despacháveis.

- FEDs não formadoras de rede - necessitam uma referência de tensão e frequência para injetar potência na rede. Geralmente estão associadas às fontes não despacháveis, como é o caso de painéis fotovoltaicos ou turbinas eólicas de pequeno porte.

Esta classificação das unidades de geração norteia a decisão pela escolha da estratégia de controle mais apropriada para uma determinada FED acoplada à rede através de um inversor.

Neste trabalho, optou-se por investigar duas estratégias de controle para os inversores das FEDs formadoras de rede: Controle droop e Controle Droop $F$. Estas 
estratégias de controle apresentam a vantagem de possibilitar o paralelismo de inversores sem auxilio de uma conexão extra entre os mesmos, pois embora atualmente existam diversas técnicas propostas e validadas para operação estável de inversores em paralelo com comunicação (mestre-escravo, central de limite, compartilhamento de corrente média, cadeia circular e sinais de modo comum) [46], o paralelismo sem comunicação aumenta a confiabilidade do sistema como um todo, pois elimina a probabilidade de perda ou erro de comunicação entre as unidades, aumentando a versatilidade e a modularidade do sistema. Para o caso das FEDs não formadoras de rede optou-se por investigar a estratégia de Controle $P$ - $Q$, por ser o método mais amplamente utilizado para a conexão de fontes não despacháveis na rede, método que também dispensa o uso de comunicação extra entre os inversores.

Embora existam diferenças entre as estratégias de controle, o esquema básico de conexão dos inversores e similar para todos os casos e se mostra na Figura 3.9.

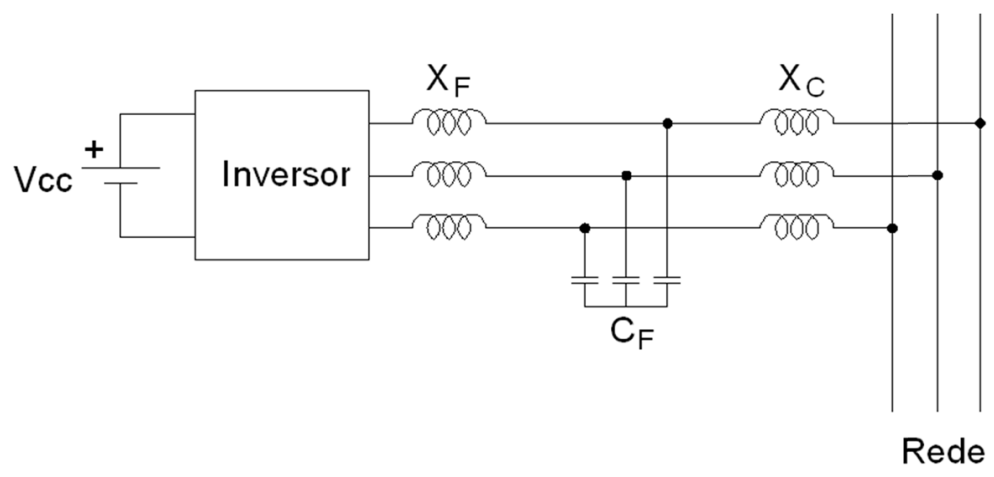

Figura 3.9 - Esquema Básico de conexão dos Inversores

Como se observa, os inversores são acoplados à rede através de uma interface constituída por um indutor de acoplamento $\left(X_{C}\right)$ e um filtro passa-baixa indutivocapacitivo $\left(X_{F}\right.$ e $\left.C_{F}\right)$ para atenuar as altas frequências de chaveamento. $O$ indutor efetivamente desacopla o inversor da rede, permitindo a regulação de potência e/ou tensão no ponto de conexão com a rede. O inversor trifásico 3-pernas do tipo VSC é composto por chaves autocomutadas, em geral IGBTs (Insulated Gate Bipolar Transistor), controladas por lógica PWM. Neste trabalho os inversores são modelados unicamente pelas suas funções de controle, sendo que os rápidos 
transitórios de chaveamento, harmônicos e perdas são negligenciados. O motivo desta simplificação se deve à rápida resposta dos inversores, que podem ser modelados do ponto de vista da rede como fontes de tensão de CA controláveis. Este é um procedimento geralmente adotado no estudo da estabilidade dinâmica de sistemas de potência que envolve conversores eletrônicos [47].

\subsubsection{Controle $P-Q$}

Como mencionado anteriormente, este tipo de controle é utilizado nas FEDs não formadoras de rede. Este controle condiciona o inversor a injetar toda a potência disponibilizada pela fonte de energia primária numa rede já energizada. A quantidade de potência reativa injetada pode ser localmente definida pelo controlador local UCM ou centralmente pelo controlador CCMG. O diagrama de blocos de um inversor com estratégia de Controle P-Q se mostra na Figura 3.10 [48].

O desenvolvimento das equações diferenciais e algébricas que descrevem o comportamento de cada bloco se mostra a seguir. Todas as equações estão expressas em pu, no mesmo sistema utilizado para modelar a máquina síncrona.

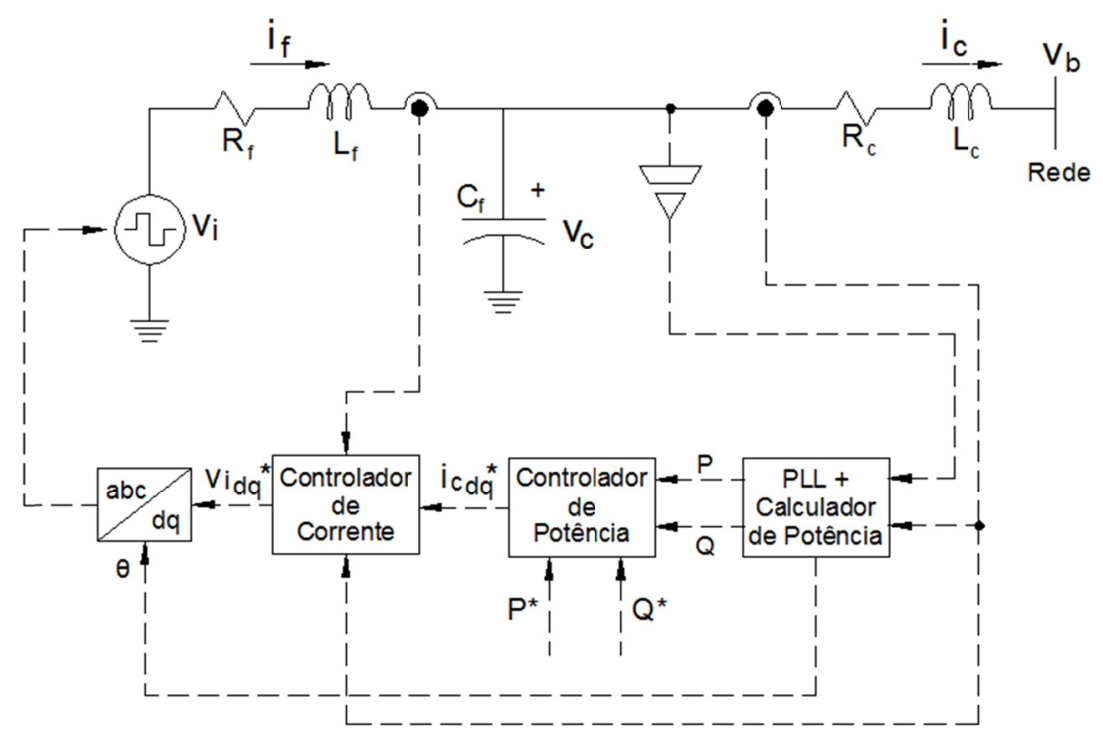

Figura 3.10 - Diagrama de blocos de um Inversor com Controle P-Q 


\subsubsection{PLL + Calculador de Potência}

A Figura 3.11 mostra o diagrama esquemático do bloco denominado PLL+Calculador de Potência [48]. Como o nome indica, este bloco está constituído por um PLL (Phase Locked Loop) atrelado na tensão $v_{c}$ e por um bloco (Calculador de Potência) responsável por estimar a potência ativa e reativa na saída do inversor.

O PLL utilizado aqui se baseia num circuito de malha fechada, que busca o ângulo de fase da tensão através da anulação da componente de quadratura no ponto de conexão, por meio do controle do ângulo $\theta$ utilizado para as transformações d-q (i.e., $v_{c q}$ tende a zero em regime).

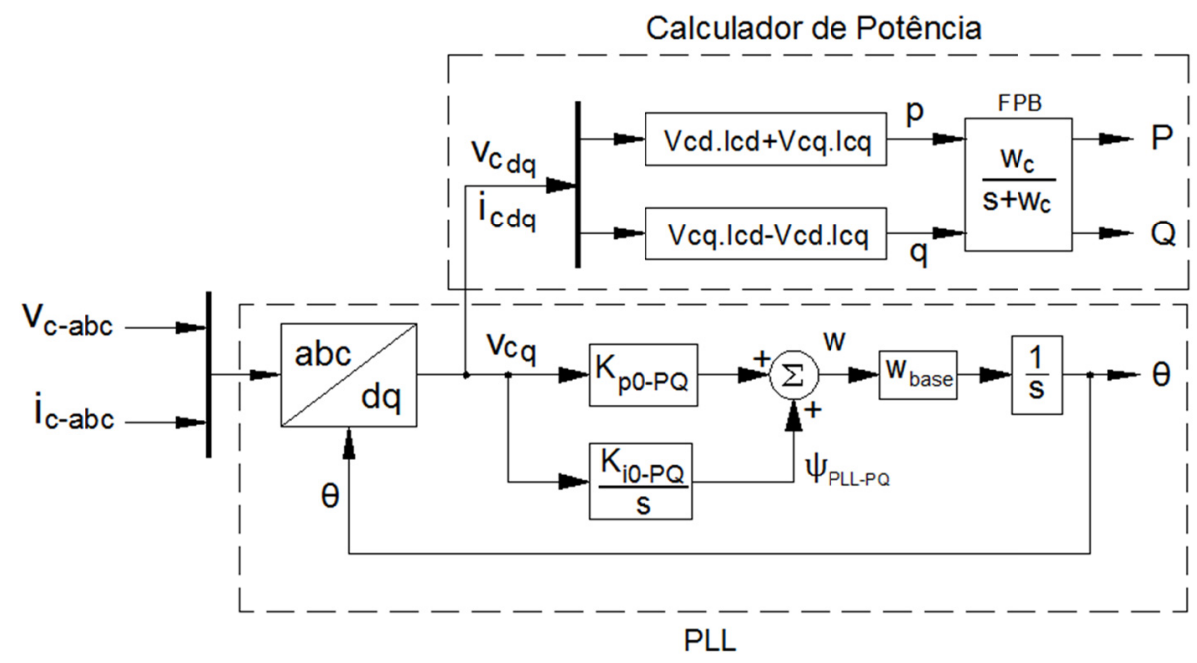

Figura 3.11 - Diagrama de blocos do PLL+Calculador de Potência-Controle P-Q

Da Figura 3.11, as equações que descrevem o comportamento do PLL são:

$$
\begin{gathered}
\psi_{P L L-P Q}=K_{i 0-P Q} \cdot v_{c q} \\
w=K_{p 0-P Q} \cdot v_{c q}+\psi_{P L L-P Q} \\
\dot{\theta}=w_{\text {base }} . w
\end{gathered}
$$


Onde:

$$
\begin{array}{ll}
w & =\text { velocidade angular em pu da referência local d-q. } \\
\theta & =\text { ângulo de fase da tensão } v_{c} \\
K_{p o-P Q} \text { e } K_{i o-P Q}= & \text { ganhos proporcional e integral respectivamente do PI da } \\
& \text { malha de controle do PLL. }
\end{array}
$$

Já o Calculador de Potência faz uso da teoria de potência instantânea conhecida como teoria p-q. Esta teoria pode ser aplicada tanto a situações de regime permanente como em transitórios e na presença de harmônicos [49]. O cálculo da potência ativa e reativa instantânea $(p$ e $q)$ se realizam através da decomposição vetorial no eixo direto e de quadratura da tensão $v_{c}$ e da corrente $i_{c}$, como mostrado na Figura 3.11, sendo que a componente zero é desprezada por se tratar de um sistema trifásico a três fios. Assim sendo, os valores instantâneos de potência ativa e reativa em pu podem ser calculados pelas seguintes expressões:

$$
\begin{aligned}
& p=v_{c d} \cdot i_{c d}+v_{c q} \cdot i_{c q} \\
& q=v_{c q} \cdot i_{c d}-v_{c d} \cdot i_{c q}
\end{aligned}
$$

Para obter as potências correspondentes à componente fundamental $(P$ e $Q)$, os valores instantâneos de potência ativa e reativa são passados através de um filtro passa-baixa (FPB) de primeira ordem, com frequência de corte $w_{c}$.

Da Figura 3 11, as equações que descrevem o comportamento do Calculador de Potência são:

$$
\begin{aligned}
& \dot{P}=-w_{c} \cdot P+w_{c} \cdot v_{c d} \cdot i_{c d}+w_{c} \cdot v_{c q} \cdot i_{c q} \\
& \dot{Q}=-w_{c} \cdot Q+w_{c} \cdot v_{c q} \cdot i_{c d}-w_{c} \cdot v_{c d} \cdot i_{c q}
\end{aligned}
$$




\subsubsection{Controlador de Potência}

A Figura 3.12 mostra o diagrama esquemático do bloco denominado Controlador de Potência [48]. Este bloco realiza o controle vetorial de corrente, permitindo que a potência ativa e reativa sejam controladas de forma desacoplada uma da outra. $A$ razão para isto pode ser explicada através da análise da eq. (3.47) e eq. (3.48). Na ausência da componente $v_{c q}$ (PLL atrelado na tensão de saída $v_{c}$ ), pode-se observar que a potência ativa é unicamente função da corrente de eixo direto, enquanto a potência reativa é função unicamente da corrente de eixo de quadratura, como mostram as expressões abaixo:

$$
\begin{gathered}
p_{\left.\right|_{v_{c q=0}}}=v_{c d} \cdot i_{c d} \\
q_{\left.\right|_{v_{c q=0}}}=-v_{c d} \cdot i_{c q}
\end{gathered}
$$

Consequentemente, o rastreamento dos valores de referência de potência ativa e reativa $\left(P^{*}, Q^{*}\right)$ impostos externamente, pode ser realizado de forma desacoplada através do controle dos valores de referência das correntes $i_{c d}{ }^{*}$ e $i_{c q}{ }^{*}$, utilizando-se para isso dois controladores PI como mostrado na Figura 3.12.
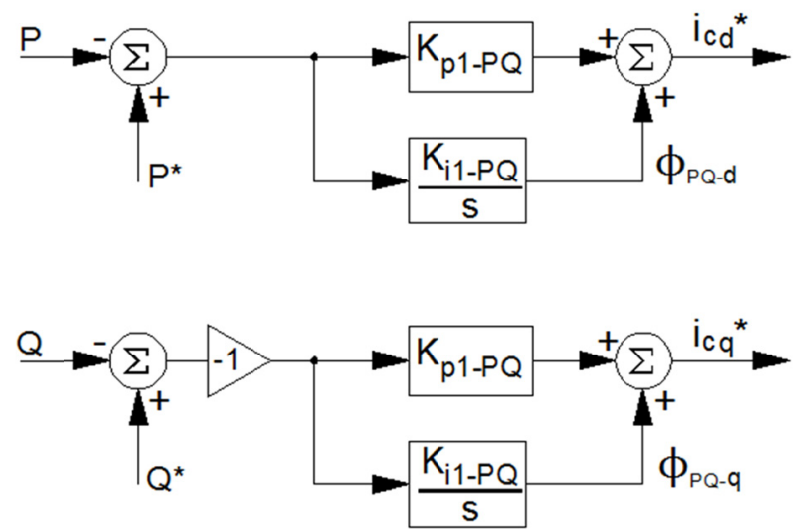

Figura 3.12 - Diagrama de Blocos do Controlador de Potência - Controle P-Q 
As equações que descrevem o comportamento dinâmico do Controlador de Potência são:

$$
\begin{gathered}
\emptyset_{P Q-d} \cdot K_{i 1-P Q} \cdot\left(P^{*}-P\right) \\
\emptyset_{P Q-q}^{\cdot}=-K_{i 1-P Q} \cdot\left(Q^{*}-Q\right) \\
i_{c d}{ }^{*}=K_{p 1-P Q} \cdot\left(P^{*}-P\right)+\emptyset_{P Q-d} \\
i_{c q}{ }^{*}=-K_{p 1-P Q} \cdot\left(Q^{*}-Q\right)+\emptyset_{P Q-q}
\end{gathered}
$$

Onde

$K_{p 1-P Q}$ e $K_{i 1-P Q} \quad=$ ganhos proporcional e integral respectivamente do $\mathrm{PI}$ do Controlador de Potência.

\subsubsection{Controlador de Corrente}

A Figura 3.13 mostra o diagrama esquemático do bloco denominado Controlador de Corrente [48]. As entradas do bloco correspondem aos valores de referência das correntes $i_{c d}{ }^{*}$ e $i_{c q}{ }^{*}$ fornecidos pelo Controlador de Potência. Se considerarmos que a corrente no capacitor do filtro é de alta frequência, em termos de potência exportada na frequência fundamental, a corrente de saída $i_{c}$ e a corrente no indutor do filtro $i_{f}$ são essencialmente iguais [48]. Assim, as correntes direta e de quadratura medidas $i_{f d}$ e $i_{f q}$ podem ser diretamente comparadas com os valores de referência $i_{c d}{ }^{*}$ e $i_{c q}{ }^{*}$. A diferença constitui o sinal de erro que é processado pelos Pls.

Para obter o valor de tensão a ser sintetizado pelo inversor $\left(v_{i d} e v_{i q}\right)$, um termo de compensação é adicionado à saída de cada PI, de forma a minimizar o acoplamento cruzado entre as correntes de eixo direto e de quadratura. 


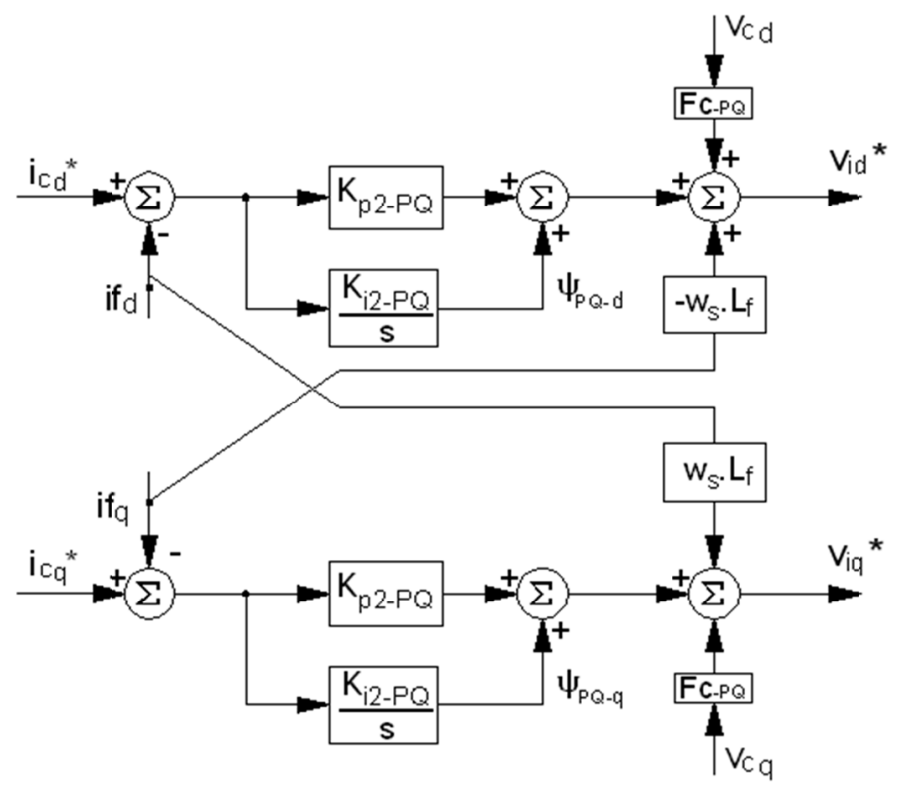

Figura 3.13 - Diagrama de Blocos do Controlador de Corrente- Controle P-Q

As equações que modelam o comportamento do Controlador de Corrente são:

$$
\begin{gathered}
\psi_{P Q-d}^{\cdot}=K_{i 2-P Q \cdot} \cdot\left(i_{c d}{ }^{*}-i_{f d}\right) \\
\psi_{P Q-q}^{\cdot}=K_{i 2-P Q \cdot} \cdot\left(i_{c q}{ }^{*}-i_{f q}\right) \\
v_{i d}{ }^{*}=K_{p 2-P Q} \cdot\left(i_{c d}{ }^{*}-i_{f d}\right)+\psi_{P Q-d}+F_{c-P Q} \cdot v_{c d}-w_{s} \cdot L_{f} \cdot i_{f q} \\
v_{i q}{ }^{*}=K_{p 2-P Q} \cdot\left(i_{c q}{ }^{*}-i_{f q}\right)+\psi_{P Q-q}+F_{c-P Q} \cdot v_{c q}+w_{s} \cdot L_{f} \cdot i_{f d}
\end{gathered}
$$

Onde

$K_{p 2-P Q}$ e $K_{i 2-P Q} \quad=$ ganhos proporcional e integral respectivamente do $\mathrm{Pl}$ do Controlador de Corrente.

$F_{C-P Q} \quad=$ ganho feedforward da malha de corrente.

$L_{f} \quad$ = indutância do filtro LC 


\subsubsection{Interface do Inversor com a rede}

As EDOs que governam o comportamento da interface do inversor com a rede (indutor de acoplamento + filtro), podem ser formuladas aplicando-se a primeira e segunda lei de Kirchhoff ao circuito mostrado na Figura 3.10. Em termos das componentes d-q, estas equações em pu são:

$$
\begin{gathered}
\dot{l}_{c d}=w_{\text {base }}\left[-\frac{R_{c}}{L_{c}} \cdot i_{c d}+\frac{1}{L_{c}} \cdot v_{c d}-\frac{1}{L_{c}} \cdot v_{b d}+w \cdot i_{c q}\right] \\
\dot{l}_{c q}=w_{\text {base }}\left[-\frac{R_{c}}{L_{c}} \cdot i_{c q}+\frac{1}{L_{c}} \cdot v_{c q}-\frac{1}{L_{c}} \cdot v_{b q}-w \cdot i_{c d}\right] \\
\dot{l}_{f d}=w_{b a s e}\left[-\frac{R_{f}}{L_{f}} \cdot i_{f d}-\frac{1}{L_{f}} \cdot v_{c d}+\frac{1}{L_{f}} \cdot v_{i d}+w \cdot i_{f q}\right] \\
\dot{l}_{f q}=w_{b a s e}\left[-\frac{R_{f}}{L_{f}} \cdot i_{f q}-\frac{1}{L_{f}} \cdot v_{c q}+\frac{1}{L_{f}} \cdot v_{i q}-w \cdot i_{f d}\right] \\
v_{c q}^{\cdot}=w_{b a s e}\left[\frac{1}{C_{f}} \cdot i_{f q}-\frac{1}{C_{f}} \cdot i_{c q}-w \cdot v_{c d}\right] \\
v_{c d}^{\cdot}=w_{b a s e}\left[\frac{1}{C_{f}} \cdot i_{f d}-\frac{1}{C_{f}} \cdot i_{c d}+w \cdot v_{c q}\right]
\end{gathered}
$$

Onde,

$L_{c} \quad$ =indutância de acoplamento.

$C_{f} \quad=$ capacitância do filtro LC

$R_{c}$ e $R_{f} \quad$ = resistência inerente ao indutor de acoplamento e indutor do filtro LC respectivamente. 


\subsubsection{Transformação de coordenadas}

Para expressar as variáveis elétricas de entrada/saída do modelo do inversor, na referência comum $\mathrm{D}-\mathrm{Q}$, é necessário definir um ângulo $\delta$ relacionando a referência local d-q com a referência comum através da seguinte expressão (ver Figura 3.4):

$$
\dot{\delta}=w_{\text {base }} \cdot\left(w-w_{\text {com }}\right)
$$

As variáveis elétricas de entrada do modelo correspondem às tensões de fase verificadas no barramento de conexão do inversor com a rede, transladadas para a referência comum $\left(v_{b D Q}\right)$, enquanto que as variáveis elétricas de saída correspondem as correntes injetadas pelo inversor na rede, também transladadas para a referência comum $\left(i_{c D Q}\right)$.

\subsubsection{Modelo Completo do Inversor P-Q}

As equações (3.44) à (3.67) descrevem a dinâmica do Controle P-Q, com: $\theta, \psi_{P L L-P Q}, \delta, P, Q, \phi_{P Q-d}, \phi_{P Q-q}, \psi_{P Q-d}, \psi_{P Q-q}, i_{f d}, i_{f q}, v_{c d}, v_{c q}, i_{c d}$ e $i_{c q}$ como variáveis de estado. Porém, as derivadas destas variáveis aparecem nestas equações como função de: $w, i_{c d}{ }^{*}, i_{c q}{ }^{*}, v_{i d}{ }^{*} e v_{i q}{ }^{*}$ que não são nem entradas nem variáveis de estado. Consequentemente, é necessário eliminar estas variáveis das respectivas equações de estado. Por outra parte, as entradas e saídas do sistema estão na referência local, sendo necessário expressá-las na referência comum D-Q, através das eq.(3.19) e (3.20). Realizando as devidas substituições e linearizando as expressões resultantes por expansão em série de Taylor, se tem o modelo completo no espaço de estados do Controle $P-Q$, mostrado a seguir:

$$
\left[\Delta \dot{X}_{P Q}\right]=\left[A_{P Q}\right] \cdot\left[\Delta X_{P Q}\right]+\left[B 1_{P Q}\right] \cdot\left[\Delta v_{b D Q}\right]+\left[B 2_{P Q}\right] \cdot \Delta w_{c o m}
$$


Onde,

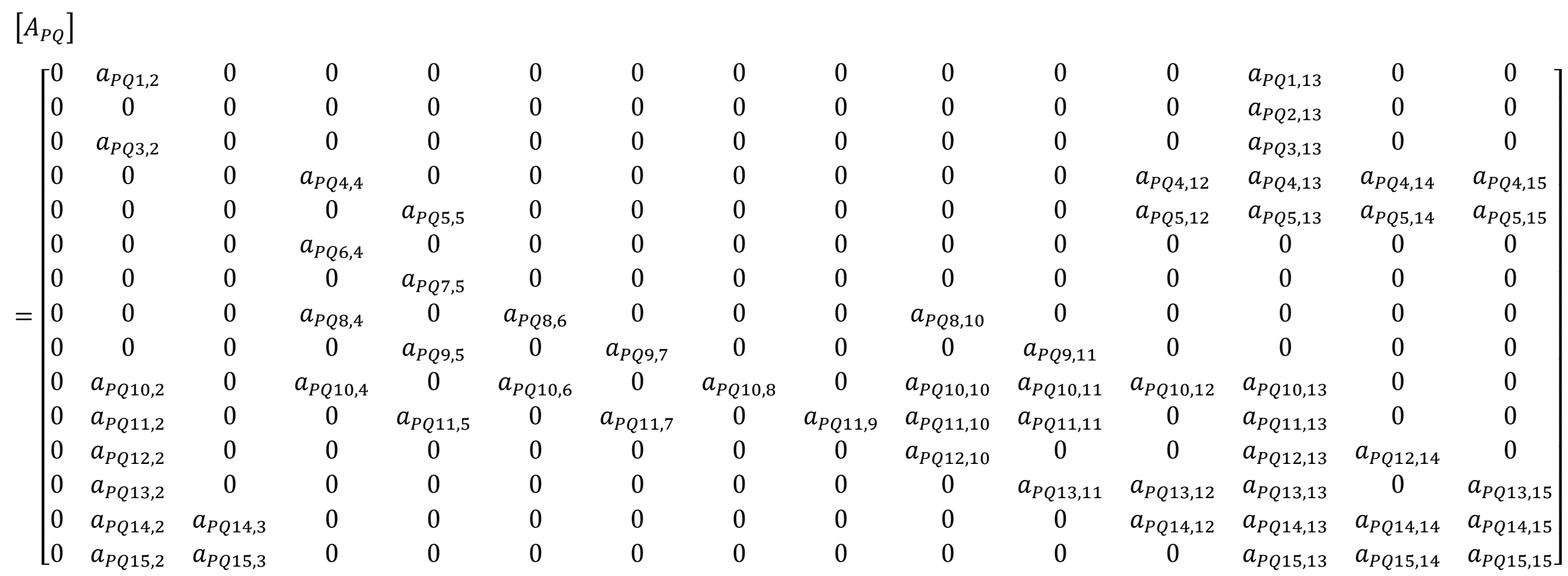




$$
\left[\Delta X_{P Q}\right]=\left[\begin{array}{c}
\Delta \theta \\
\Delta \psi_{P L L-P Q} \\
\Delta \delta \\
\Delta P \\
\Delta Q \\
\Delta \phi_{P Q-d} \\
\Delta \phi_{P Q-q} \\
\Delta \psi_{P Q-d} \\
\Delta \psi_{P Q-q} \\
\Delta i f_{d} \\
\Delta i f_{q} \\
\Delta v_{c d} \\
\Delta v_{c q} \\
\Delta i_{c d} \\
\Delta i_{c q}
\end{array}\right]
$$

$$
\left[B 2_{P Q}\right]=\left[\begin{array}{c}
0 \\
0 \\
b 2_{P Q 3,1} \\
0 \\
0 \\
0 \\
0 \\
0 \\
0 \\
0 \\
0 \\
0 \\
0 \\
0 \\
0
\end{array}\right]
$$

$$
\begin{aligned}
a_{P Q 2,13} & =K_{i 0-P Q} \\
a_{P Q 4,4} & =-w_{c} . \\
a_{P Q 4,14} & =v_{c d 0} \cdot w_{c} \\
a_{P Q 5,12} & =-i_{c q 0} \cdot w_{c} \\
a_{P Q 5,15} & =-v_{c d 0} \cdot w_{c}
\end{aligned}
$$

$$
\begin{aligned}
& a_{P Q 6,4}=-K_{i 1-P Q} \\
& a_{P Q 7,5}=K_{i 1-P Q} \\
& a_{P Q 8,4}=-K_{p 1-P Q} \cdot K_{i 2-P Q} \\
& a_{P Q 8,6}=K_{i 2-P Q} \\
& a_{P Q 8,10}=-K_{i 2-P Q} \\
& a_{P Q 9,5}=K_{p 1-P Q} \cdot K_{i 2-P Q} \\
& a_{P Q 9,7}=K_{i 2-P Q} \\
& a_{P Q 9,11}=-K_{i 2-P Q} \\
& a_{P Q 10,2}=w_{\text {base }} \cdot i_{f q 0}
\end{aligned}
$$

$$
\begin{aligned}
a_{P Q 10,4} & =\frac{w_{\text {base }} \cdot K_{p 1-P Q} \cdot K_{p 2-P Q}}{-L_{f}} \\
a_{P Q 10,8} & =\frac{w_{\text {base }}}{L_{f}} \\
a_{P Q 10,11} & =w_{\text {base }}\left(w_{0}-w_{s}\right) \\
a_{P Q 10,13} & =w_{\text {base }} i_{f q 0} K_{p 0-P Q} \\
a_{P Q 11,5} & =\frac{w_{\text {base }} K_{p 1-P Q} K_{p 2-P Q}}{L_{f}} \\
a_{P Q 11,9} & =\frac{w_{\text {base }}}{L_{f}}
\end{aligned}
$$$$
a_{P Q 10,6}=\frac{w_{\text {base }} \cdot K_{p 2-P Q}}{L_{f}}
$$$$
a_{P Q 10,10}=\frac{w_{\text {base }}\left(K_{p 2-P Q}+R_{f}\right)}{-L_{f}}
$$

$$
\begin{aligned}
a_{P Q 10,12} & =\frac{w_{\text {base }}\left(F_{c-P Q}-1\right)}{L_{f}} \\
a_{P Q 11,2} & =-w_{\text {base }} \cdot i_{f d 0} \\
a_{P Q 11,7} & =\frac{w_{\text {base }} K_{p 2-P Q}}{L_{f}}
\end{aligned}
$$




$$
\begin{aligned}
& a_{P Q 11,10}=w_{\text {base }}\left(w_{0}-w_{s}\right) \\
& a_{P Q 11,13}=w_{\text {base }}\left[\frac{F_{c-P Q}-1}{L_{f}}-i_{f d 0} \cdot K_{p 0-P Q}\right] \\
& a_{P Q 11,11}=\frac{w_{\text {base }}\left(K_{p 2-P Q}-R_{f}\right)}{-L_{f}} \\
& a_{P Q 12,2}=w_{\text {base }} \cdot v_{c q 0} \\
& a_{P Q 12,10}=\frac{w_{\text {base }}}{C_{f}} \\
& a_{P Q 12,14}=-\frac{w_{\text {base }}}{C_{f}} \\
& a_{P Q 12,13}=w_{\text {base }}\left(w_{0}+K_{p 0-P Q} \cdot v_{c q 0}\right) \\
& a_{P Q 13,2}=-w_{\text {base }} \cdot v_{c d 0} \\
& a_{P Q 13,12}=-w_{\text {base }} \cdot w_{0} \\
& a_{P Q 13,11}=\frac{w_{\text {base }}}{C_{f}} \\
& a_{P Q 13,13}=-w_{\text {base }} \cdot K_{p 0-P Q} \cdot v_{c d 0} \\
& a_{P Q 13,15}=\frac{-w_{\text {base }}}{C_{f}} \\
& a_{P Q 14,3}=\frac{w_{\text {base }}\left[v_{b Q 0} \cos \left(\delta_{0}\right)-v_{b D 0} \operatorname{sen}\left(\delta_{0}\right)\right]}{L_{c}} \\
& a_{P Q 14,2}=w_{\text {base }} \cdot i_{c q 0} \\
& a_{P Q 14,12}=\frac{w_{\text {base }}}{L_{c}} \\
& a_{P Q 14,14}=\frac{-w_{\text {base }} \cdot R_{c}}{L_{c}} \\
& a_{P Q 14,13}=w_{\text {base }} \cdot i_{c q 0} \cdot K_{p 0-P Q} \\
& a_{P Q 14,15}=w_{\text {base }} \cdot w_{0} \\
& a_{P Q 15,2}=-w_{\text {base }} \cdot i_{c d 0} \\
& a_{P Q 15,3}=\frac{w_{b a s e}\left[v_{b D 0} \cos \left(\delta_{0}\right)+v_{b Q 0} \operatorname{sen}\left(\delta_{0}\right)\right]}{L_{c}} \\
& a_{P Q 15,13}=w_{\text {base }}\left[\frac{1}{L_{c}}-i_{c d 0} \cdot K_{p 0-P Q}\right] \\
& a_{P Q 15,14}=-w_{\text {base }} \cdot w_{0} \\
& a_{P Q 15,15}=-w_{\text {base }} \cdot \frac{R_{c}}{L_{c}} \\
& \begin{array}{ll}
b 1_{P Q 14,1}=-\frac{w_{\text {base }}}{L_{c}} \cdot \cos \left(\delta_{0}\right) & b 1_{P Q 14,2}=-\frac{w_{\text {base }}}{L_{c}} \cdot \operatorname{sen}\left(\delta_{0}\right) \\
b 1_{P Q 15,1}=\frac{w_{\text {base }}}{L_{c}} \cdot \operatorname{sen}\left(\delta_{0}\right) & b 1_{P Q 15,2}=-\frac{w_{\text {base }}}{L_{c}} \cdot \cos \left(\delta_{0}\right) \\
b 2_{P Q 3,1}=-w_{\text {base }} & \Delta v_{b D Q}=\left[\begin{array}{l}
\Delta v_{b D} \\
\Delta v_{b Q}
\end{array}\right]
\end{array}
\end{aligned}
$$

As equações relacionando as saídas do modelo (i.e., as correntes $i_{c D}$ e $i_{c Q}$ ) com as variáveis de estado são:

$$
\left[\Delta i_{C D Q}\right]=\left[C 1_{P Q}\right] \cdot\left[\Delta X_{P Q}\right]
$$

\section{Onde}

$$
\left[C 1_{P Q}\right]=\left[\begin{array}{lllllllllllllll}
0 & 0 & c 1_{P Q 1,3} & 0 & 0 & 0 & 0 & 0 & 0 & 0 & 0 & 0 & 0 & c 1_{P Q 1,14} & c 1_{P Q 1,15} \\
0 & 0 & c 1_{P Q 2,3} & 0 & 0 & 0 & 0 & 0 & 0 & 0 & 0 & 0 & 0 & c 1_{P Q 2,14} & c 1_{P Q 2,15}
\end{array}\right]
$$




$$
\begin{array}{lrl}
c 1_{P Q 1,3}=-i_{c q 0} \cdot \cos \left(\delta_{0}\right)-i_{c d 0} \cdot \operatorname{sen}\left(\delta_{0}\right) & c 1_{P Q 1,14}=\cos \left(\delta_{0}\right) \\
c 1_{P Q 1,15}=-\operatorname{sen}\left(\delta_{0}\right) & c 1_{P Q 2,3}=i_{c d 0} \cdot \cos \left(\delta_{0}\right)-i_{c q 0} \cdot \operatorname{sen}\left(\delta_{0}\right) \\
c 1_{P Q 2,14}=\operatorname{sen}\left(\delta_{0}\right) & c 1_{P Q 2,15}=\cos \left(\delta_{0}\right)
\end{array}
$$

\subsubsection{Controle Droop}

Este tipo de controle é utilizado nas fontes formadoras de rede e permite ao inversor emular o controle primário de frequência das máquinas síncronas dos grandes sistemas de potência, possibilitando o paralelismo de inversores sem comunicação. As variações de potência ativa são partilhadas em função da característica de estatismo "potência-ativa/frequência" dada a cada inversor, enquanto a potência reativa é partilhada de forma semelhante, pelo estatismo assiociado à "potênciareativa/tensão". Os dois tipos de estatismo apresentam uma inclinação decrescente (droop), o que significa que um incremento de carga ativa $(\Delta P)$ vem acompanhado de um decremento na velocidade ${ }^{11}$ (frequência) da unidade, e um incremento de carga reativa $(\Delta Q)$ vem acompanhado de um decremento na tensão de saída do inversor, como se mostra na Figura 3.14.

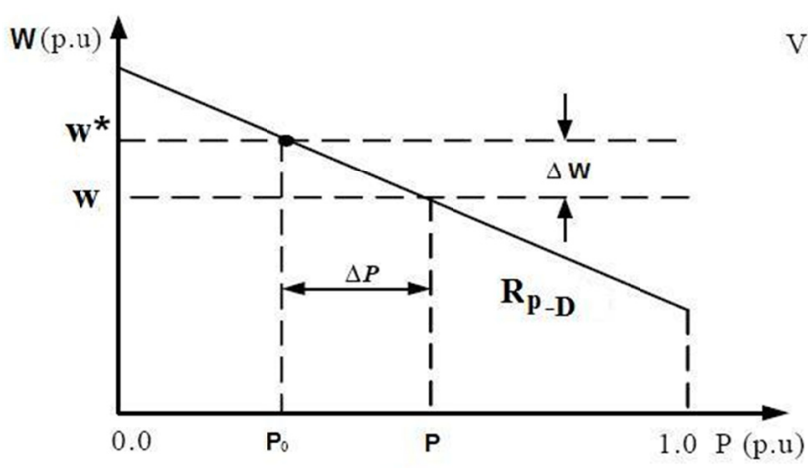

(a)

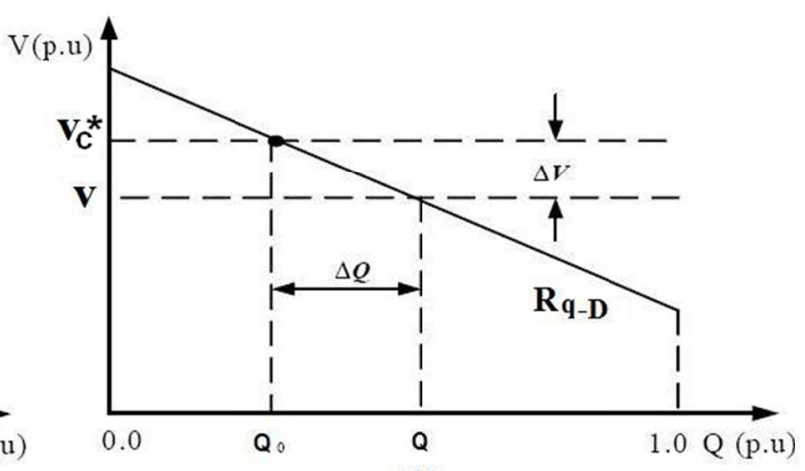

(b)

Figura 3.14 - Característica de estatismo (a) potência-ativa/frequência (b) potênciareativa/tensão

\footnotetext{
11 Neste trabalho se usam os termos velocidade e frequência indistintamente porque descrevem quantidades que são proporcionais.
} 
Onde $P$ e $Q$ são as potências ativa e reativa à saída do inversor, $R_{p-D}$ e $R_{q-D}$ são os droops (valores positivos) de potência ativa e reativa respectivamente e $w^{*}$ e $v_{c}{ }^{*}$ são respectivamente os valores de frequência e tensão que o inversor deve apresentar em situação de operação nominal. Os valores de droop $\left(R_{p-D}\right.$ e $\left.R_{q-D}\right)$ assim como os valores de frequência e tensão nominais $\left(w^{*}\right.$ e $\left.v_{c}{ }^{*}\right)$ podem ser definidos localmente pelo UCM ou centralmente pelo CCMG.

O diagrama de blocos de um inversor com estratégia de Controle Droop se mostra na Figura 3.15 [27]. O desenvolvimento das equações que descrevem o comportamento de cada bloco se mostra a seguir. Todas as equações estão expressas em pu, no mesmo sistema utilizado para modelar a máquina síncrona.

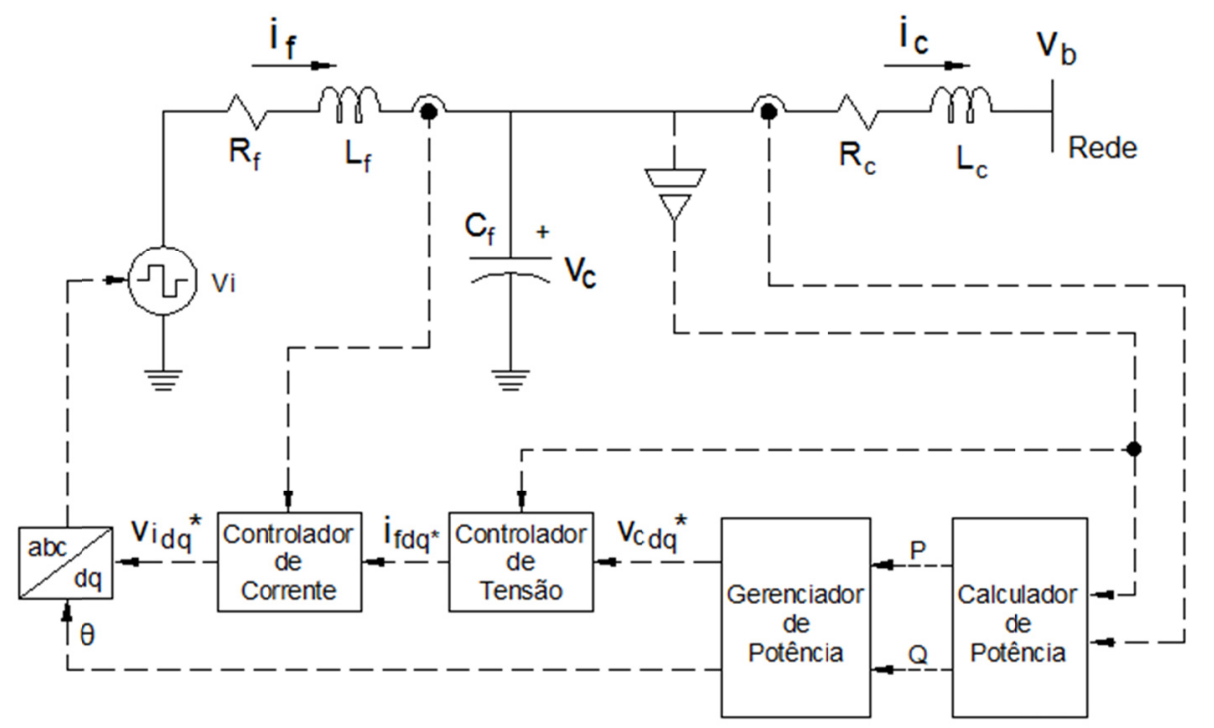

Figura 3.15 - Diagrama de blocos de um Inversor com Controle Droop

\subsubsection{Calculador de Potência}

O Calculador de Potência do Controle Droop, de forma similar ao seu equivalente do Controle $P-Q$, faz uso da teoria de potência instantânea para estimar a potência ativa e reativa na saída do inversor, porém neste caso, o ângulo de referência $\theta$, necessário para as transformações abc/dq, não é gerado por um PLL e sim pelo bloco denominado Gerenciador de Potência como será visto mais adiante. A Figura 3.16 mostra o diagrama esquemático deste bloco [27]. 


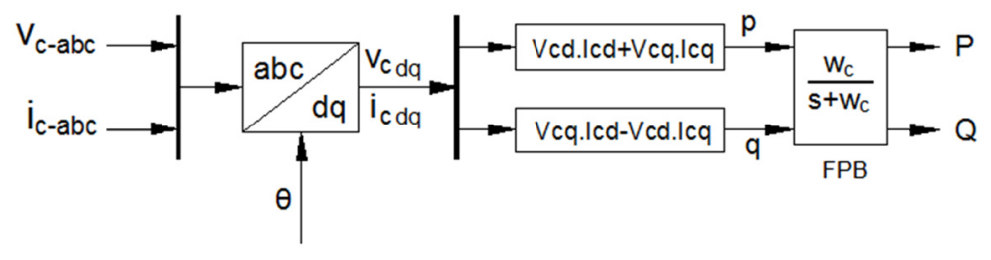

Figura 3.16 - Diagrama de blocos do Calculador de Potência - Controle Droop

As EDOs que modelam o comportamento dinâmico do Calculador de Potência são as mesmas do seu equivalente no Controle P-Q. (eq. (3.49) e eq. (3.50)).

\subsubsection{Gerenciador de Potência}

A Figura 3.17 mostra o diagrama esquemático do Gerenciador de Potência [27]. Como se observa, o intercâmbio de potência ativa entre o inversor e a rede é gerenciado através do controle do ângulo de fase $\theta$ da tensão de referência $v_{c}{ }^{*}$, enquanto o intercâmbio de potência reativa é gerenciado pelo controle da magnitude da tensão de referência $v_{c}{ }^{*}$. A estratégia de controle escolhida alinha a tensão de referência $v_{c}{ }^{*}$ com o eixo direto da referência local do inversor, consequentemente a componente de quadratura de $v_{c}{ }^{*}$ é nula $\left(v_{c q}{ }^{*}=0\right)$.

Se fizermos uma analogia entre o ângulo de fase $\theta$ do inversor e o ângulo de carga de um gerador síncrono, observamos que o comportamento da uma máquina síncrona é efetivamente emulado através desta técnica de controle. No caso do inversor (assim como da máquina síncrona) é possível controlar a potência ativa injetada variando transitoriamente a frequência da tensão de saída, pois se um inversor tem temporariamente uma frequência superior que o restante do sistema, a sua posição angular relativa $(\theta)$ se incrementa, transferindo parte da carga elétrica do sistema para si mesmo. Porém, devido à característica decrescente do estatismo (droop), esta ação reduz a sua própria frequência e consequentemente diminui a separação angular até se alcançar o equilíbrio. Um raciocínio similar pode ser feito para o caso da potência reativa, sendo neste caso possível controlar a potência reativa injetada através da variação do módulo da tensão de saída do inversor. Embora esta relação (potência ativa/frequência, potência reativa/tensão) seja 
verificada unicamente em sistemas predominantemente indutivos, trabalhos nesta área [50] tem mostrado a aplicabilidade desta técnica de controle em sistemas predominantemente resistivos.

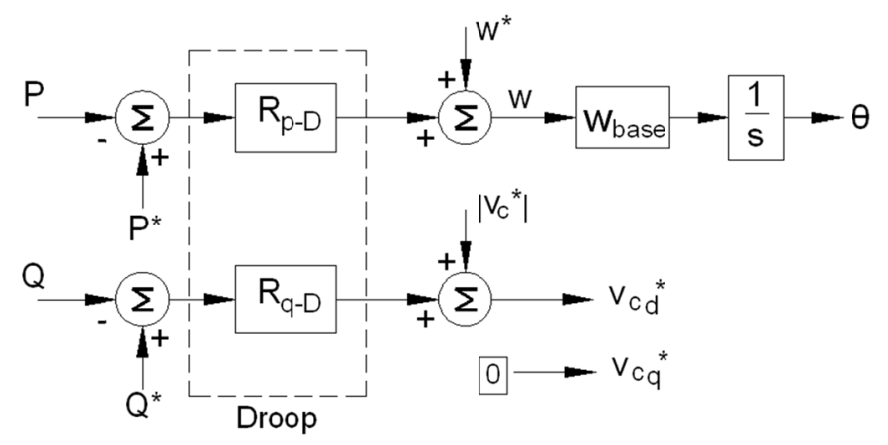

Figura 3.17 - Diagrama de blocos do Gerenciador de Potência - Controle Droop

As equações que descrevem o comportamento do Gerenciador de Potência são:

$$
\begin{gathered}
w=w^{*}+R_{p-D}\left(P^{*}-P\right) \\
\dot{\theta}=w_{\text {base }} \cdot w \\
v_{c d}{ }^{*}=\left|v_{c}{ }^{*}\right|+R_{q-D} \cdot\left(Q^{*}-Q\right) \\
v_{c q}{ }^{*}=0
\end{gathered}
$$

\subsubsection{Controlador de Tensão}

A Figura 3.18 mostra o diagrama esquemático do bloco denominado Controlador de Tensão [27]. As entradas do bloco correspondem aos valores de referência das tensões $v_{c d}{ }^{*}$ e $v_{c q}{ }^{*}$ fornecidos pelo Gerenciador de Potência. Estes valores são comparados com as tensões $v_{c d}$ e $v_{c q}$ medidas, sendo a diferença o sinal de erro que é processado pelos Pls. 


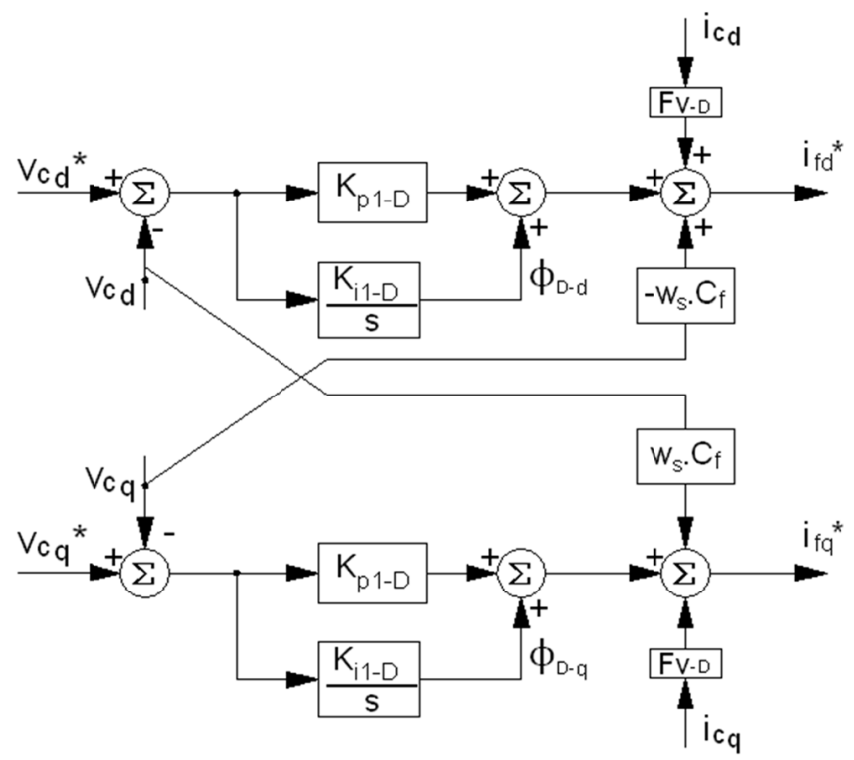

Figura 3.18 - Diagrama de blocos do Controlador de Tensão - Controle Droop

A saída do bloco constituem os valores de referência das correntes $i_{f d}{ }^{*}$ e $i_{f q}{ }^{*}$. Um termo de compensação é adicionado à saída de cada $\mathrm{PI}$, de forma a minimizar o acoplamento cruzado entre as tensões de eixo direto e de quadratura. As equações que modelam o comportamento do Controlador de Tensão são:

$$
\begin{gathered}
\emptyset_{D-d}^{\cdot}=K_{i 1-D} \cdot\left(v_{c d}{ }^{*}-v_{c d}\right) \\
\emptyset_{D-q}^{\cdot}=K_{i 1-D} \cdot\left(v_{c q}{ }^{*}-v_{c q}\right) \\
i_{f d}{ }^{*}=K_{p 1-D} \cdot\left(v_{c d}{ }^{*}-v_{c d}\right)+\emptyset_{D-d}+F_{v-D} \cdot i_{c d}-w_{s} \cdot C_{f} \cdot v_{c q} \\
i_{f q}{ }^{*}=K_{p 1-D} \cdot\left(v_{c q}{ }^{*}-v_{c q}\right)+\emptyset_{D-q}+F_{v-D} \cdot i_{c q}+w_{s} \cdot C_{f} \cdot v_{c d}
\end{gathered}
$$

Onde,

$K_{p 1-D}$ e $K_{i 1-D} \quad=$ ganhos proporcional e integral respectivamente do $\mathrm{Pl}$ do Controlador de Tensão. 
$F_{v-D} \quad=$ ganho feedforward da malha de tensão.

$C_{f} \quad=$ capacitância do filtro LC

\subsubsection{Controlador de Corrente}

A Figura 3.19 mostra o diagrama esquemático do bloco denominado Controlador de Corrente [27]. As entradas do bloco correspondem aos valores de referência das correntes $i_{f d}{ }^{*}$ e $i_{f q}{ }^{*}$ fornecidas pelo Controlador de Tensão. Estes valores são comparados com as correntes $i_{f d}$ e $i_{f q}$ medidas, sendo a diferença o sinal de erro que é processado pelos Pls.

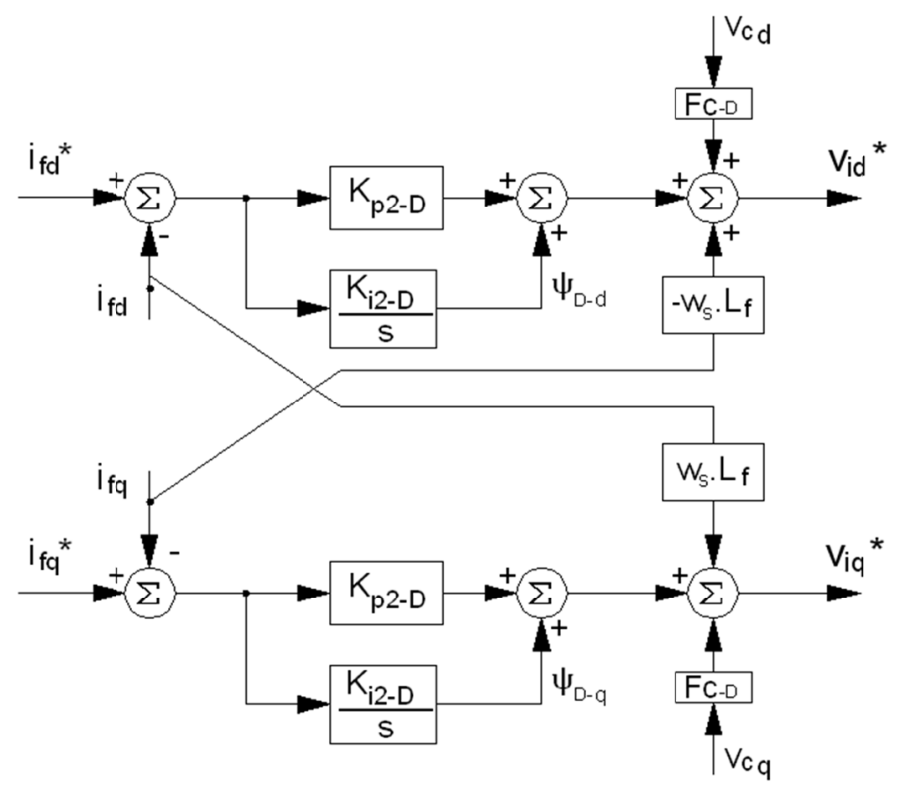

Figura 3.19 - Diagrama de blocos do Controlador de Corrente - Controle Droop

A saída do bloco constituem os valores de referência das tensões $v_{i d}{ }^{*} \mathrm{e} v_{i q}{ }^{*}$ a serem sintetizadas pelo inversor. Um termo de compensação é adicionado à saída de cada $\mathrm{PI}$, de forma a minimizar o acoplamento cruzado entre as correntes de eixo direto e de quadratura. 
As equações que modelam o comportamento do Controlador de Corrente são:

$$
\begin{gathered}
\psi_{D-d}^{\cdot}=K_{i 2-D} \cdot\left(i_{f d}{ }^{*}-i_{f d}\right) \\
\psi_{D-q}^{\cdot}=K_{i 2-D} \cdot\left(i_{f q}{ }^{*}-i_{f q}\right) \\
v_{i d}{ }^{*}=K_{p 2-D} \cdot\left(i_{f d}{ }^{*}-i_{f d}\right)+\psi_{D-d}+F_{c-D} \cdot v_{c d}-w_{s} \cdot L_{f} i_{f q} \\
v_{i q}{ }^{*}=K_{p 2-D} \cdot\left(i_{f q}{ }^{*}-i_{f q}\right)+\psi_{D-q}+F_{c-D} \cdot v_{c q}+w_{s} \cdot L_{f} i_{f d}
\end{gathered}
$$

Onde,

$K_{p 2-D}$ e $K_{i 2-D} \quad=$ ganhos proporcional e integral respectivamente do PI do Controlador de Corrente.

$F_{C-D} \quad=$ ganho feedforward da malha de corrente.

$L_{f} \quad$ = indutância do filtro LC

\subsubsection{Interface do Inversor com a rede}

As EDOs que governam o comportamento da interface do inversor com a rede são semelhantes às desenvolvidas para o Controle $P$-Q e expressas pelas eq. (3.61) à (3.66). 


\subsubsection{Transformação de coordenadas}

Para expressar as variáveis elétricas de entrada/saída do modelo do inversor, na referência comum D-Q, novamente é necessário definir um ângulo $\delta$ relacionando a referência local d-q com a referência comum através da eq. (3.67).

As variáveis elétricas de entrada do modelo correspondem às tensões de fase verificadas no barramento de conexão do inversor com a rede, transladadas para a referência comum $\left(v_{b D Q}\right)$, enquanto que as variáveis elétricas de saída correspondem as correntes injetadas pelo inversor na rede, também transladadas para a referência comum $\left(i_{c D Q}\right)$.

\subsubsection{Modelo completo do Inversor Droop}

As equações (3.49), (3.50), junto com as eq. (3.61) à eq. (3.67) e eq. (3.70) à eq. (3.81) descrevem a dinâmica do Controle Droop, com: $\theta, \delta, P, Q, \emptyset_{D-d}, \emptyset_{D-q}, \psi_{D-d}, \psi_{D-q}, i_{f d}, i_{f q}, v_{c d}, v_{c q}, i_{c d} e i_{c q}$ como variáveis de estado. Porém, as derivadas destas variáveis aparecem nestas equações como função de: $w, i_{f d}{ }^{*}, i_{f q}{ }^{*}, v_{i d}{ }^{*} e v_{i q}{ }^{*}$ que não são nem entradas nem variáveis de estado. Consequentemente, é necessário eliminar estas variáveis das respectivas equações de estado.

Por outra parte, as entradas e saídas do sistema estão na referência local, sendo necessário expressá-las na referência comum D-Q, através das eq.(3.19) e (3.20).

Realizando as devidas substituições e linearizando as expressões resultantes por expansão em série de Taylor, se tem o modelo completo no espaço de estados do Controle Droop, mostrado a seguir:

$$
\left[\Delta \dot{X}_{D}\right]=\left[A_{D}\right] \cdot\left[\Delta X_{D}\right]+\left[B 1_{D}\right] \cdot\left[\Delta v b_{D Q}\right]+\left[B 2_{D}\right] \cdot \Delta w_{c o m}
$$


Onde,

$$
\left[A_{D}\right]=\left[\begin{array}{cccccccccccccc}
0 & 0 & a_{D 1,3} & 0 & 0 & 0 & 0 & 0 & 0 & 0 & 0 & 0 & 0 & 0 \\
0 & 0 & a_{D 2,3} & 0 & 0 & 0 & 0 & 0 & 0 & 0 & 0 & 0 & 0 & 0 \\
0 & 0 & a_{D 3,3} & 0 & 0 & 0 & 0 & 0 & 0 & 0 & a_{D 3,11} & a_{D 3,12} & a_{D 3,13} & a_{D 3,14} \\
0 & 0 & 0 & a_{D 4,4} & 0 & 0 & 0 & 0 & 0 & 0 & a_{D 4,11} & a_{D 4,12} & a_{D 4,13} & a_{D 4,14} \\
0 & 0 & 0 & a_{D 5,4} & 0 & 0 & 0 & 0 & 0 & 0 & a_{D 5,11} & 0 & 0 & 0 \\
0 & 0 & 0 & 0 & 0 & 0 & 0 & 0 & 0 & 0 & 0 & a_{D 6,12} & 0 & 0 \\
0 & 0 & 0 & a_{D 7,4} & a_{D 7,5} & 0 & 0 & 0 & a_{D 7,9} & 0 & a_{D 7,11} & a_{D 7,12} & a_{D 7,13} & 0 \\
0 & 0 & 0 & 0 & 0 & a_{D 8,6} & 0 & 0 & 0 & a_{D 8,10} & a_{D 8,11} & a_{D 8,12} & 0 & a_{D 8,14} \\
0 & 0 & a_{D 9,3} & a_{D 9,4} & a_{D 9,5} & 0 & a_{D 9,7} & 0 & a_{D 9,9} & a_{D 9,10} & a_{D 9,11} & a_{D 9,12} & a_{D 9,13} & 0 \\
0 & 0 & a_{D 10,3} & 0 & 0 & a_{D 10,6} & 0 & a_{D 10,8} & a_{D 10,9} & a_{D 10,10} & a_{D 10,11} & a_{D 10,12} & 0 & a_{D 10,14} \\
0 & 0 & a_{D 11,3} & 0 & 0 & 0 & 0 & 0 & a_{D 11,9} & 0 & 0 & a_{D 11,12} & a_{D 11,13} & 0 \\
0 & 0 & a_{D 12,3} & 0 & 0 & 0 & 0 & 0 & 0 & a_{D 12,10} & a_{D 12,11} & 0 & 0 & a_{D 12,14} \\
0 & a_{D 13,2} & a_{D 13,3} & 0 & 0 & 0 & 0 & 0 & 0 & 0 & a_{D 13,11} & 0 & a_{D 13,13} & a_{D 13,14} \\
0 & a_{D 14,2} & a_{D 14,3} & 0 & 0 & 0 & 0 & 0 & 0 & 0 & 0 & a_{D 14,12} & a_{D 14,13} & a_{D 14,14}
\end{array}\right]
$$




$$
\left[\Delta X_{D}\right]=\left[\begin{array}{c}
\Delta \theta \\
\Delta \delta \\
\Delta P \\
\Delta Q \\
\Delta \emptyset_{D-d} \\
\Delta \emptyset_{D-q} \\
\Delta \psi_{D-d} \\
\Delta \psi_{D-q} \\
\Delta i_{f d} \\
\Delta i_{f q} \\
\Delta v_{c d} \\
\Delta v_{c q} \\
\Delta i_{c d} \\
\Delta i_{c q}
\end{array}\right] \quad\left[B 1_{D}\right]=\left[\begin{array}{cc}
0 & 0 \\
0 & 0 \\
0 & 0 \\
0 & 0 \\
0 & 0 \\
0 & 0 \\
0 & 0 \\
0 & 0 \\
0 & 0 \\
0 & 0 \\
0 & 0 \\
0 & 0 \\
b 1_{D 13,1} & b 1_{D 13,2} \\
b 1_{D 14,1} & b 1_{D 14,2}
\end{array}\right] \quad B 2_{D}=\left[\begin{array}{c}
0 \\
b 2_{D 1,2} \\
0 \\
0 \\
0 \\
0 \\
0 \\
0 \\
0 \\
0 \\
0 \\
0 \\
0 \\
0
\end{array}\right]
$$

Sendo,

$$
\begin{aligned}
& a_{D 1,3}=-w_{\text {base }} \cdot R_{p-D} \quad a_{D 2,3}=-w_{\text {base }} \cdot R_{p-D} \quad a_{D 3,3}=-w_{c} \\
& a_{D 3,11}=i_{c d 0} \cdot w_{c} \quad a_{D 3,12}=i_{c q 0} \cdot w_{c} \quad a_{D 3,13}=v_{c d 0} \cdot w_{c} \\
& a_{D 3,14}=v_{c q 0} \cdot w_{c} \quad a_{D 4,4}=-w_{c} \quad a_{D 4,11}=-i_{c q 0} \cdot w_{c} \\
& a_{D 4,12}=i_{c d 0} \cdot w_{c} \quad a_{D 4,13}=v_{c q 0} \cdot w_{c} \quad a_{D 4,14}=-v_{c d 0} \cdot w_{c} \\
& a_{D 5,4}=-K_{i 1-D} \cdot R_{q-D} \quad a_{D 5,11}=-K_{i 1-D} \quad a_{D 6,12}=-K_{i 1-D} \\
& a_{D 7,4}=-K_{i 2-D} K_{p 1-D} R_{q-D} \quad a_{D 7,5}=K_{i 2-D} \quad a_{D 7,9}=-K_{i 2-D} \\
& a_{D 7,11}=-K_{i 2-D} \cdot K_{p 1-D} \quad a_{D 7,12}=-C_{f} \cdot K_{i 2-D} \cdot w_{S} \quad a_{D 7,13}=F_{v-D} \cdot K_{i 2-D} \\
& a_{D 8,6}=K_{i 2-D} \quad a_{D 8,11}=C_{f} \cdot K_{i 2-D} \cdot w_{S} \quad a_{D 8,12}=-K_{i 2-D} \cdot K_{p 1-D} \\
& a_{D 8,14}=F_{v-D} \cdot K_{i 2-D} \quad a_{D 9,3}=-i_{f q 0} \cdot w_{\text {base }} \cdot R_{p-D} \quad a_{D 9,4}=\frac{K_{p 1-D} K_{p 2-D} w_{b a s e} R_{q-D}}{-L_{f}} \\
& a_{D 9,5}=\frac{K_{p 2-D} \cdot w_{\text {base }}}{L_{f}} \quad a_{D 9,7}=\frac{w_{\text {base }}}{L_{f}} \\
& a_{D 9,9}=\frac{w_{\text {base }} \cdot\left(K_{p 2-D}+R_{f}\right)}{-L f} \quad a_{D 9,10}=w_{\text {base }} \cdot\left(w_{0}-w_{s}\right) \\
& a_{D 9,11}=\frac{w_{\text {base }} \cdot\left(F_{C-D}-K_{p 1-D} \cdot K_{p 2-D}-1\right)}{a_{D 9,12}}=\frac{-w_{\text {base }} \cdot w_{s} \cdot C_{f} \cdot K_{p 2-D}}{L_{f}} \\
& a_{D 9,11}=\frac{w_{\text {base }} \cdot\left(F_{c-D}-K_{p 1-D} \cdot K_{p 2-D}-1\right)}{L_{f}} \quad a_{D 10,3}=w_{\text {base }} \cdot i_{f d 0} \cdot R_{p-D} \\
& a_{D 9,13}=\frac{w_{\text {base }} \cdot F_{v-D} \cdot K_{p 2-D}}{L_{f}} \quad a_{D 10,8}=\frac{w_{\text {base }}}{L_{f}} \\
& a_{D 10,6}=\frac{K_{p 2-D} \cdot w_{\text {base }}}{L_{f}}
\end{aligned}
$$




$$
\begin{aligned}
a_{D 10,9} & =w_{\text {base }} \cdot\left(w_{S}-w_{0}\right) \\
a_{D 10,11} & =\frac{w_{\text {base }} \cdot w_{S} \cdot C_{f} \cdot K_{p 2-D}}{L_{f}} \\
a_{D 10,14} & =\frac{w_{\text {base }} \cdot F_{v-D} \cdot K_{p 2-D}}{L_{f}} \\
a_{D 11,9} & =\frac{w_{\text {base }}}{C_{f}}
\end{aligned}
$$

$$
\begin{aligned}
a_{D 10,10} & =\frac{w_{\text {base }} \cdot\left(-K_{p 2-D}-R_{f}\right)}{L_{f}} \\
a_{D 10,12} & =\frac{w_{\text {base }} \cdot\left(F_{c-D}-K_{p 1-D} \cdot K_{p 2-D}-1\right)}{L_{f}} \\
a_{D 11,3} & =-w_{\text {base }} \cdot v_{c q 0} \cdot R_{p-D} \\
a_{D 11,12} & =w_{\text {base }} \cdot w_{0} \\
a_{D 11.13} & =-w_{\text {base }} / C_{f}
\end{aligned}
$$

$$
\begin{aligned}
& a_{D 12,3}=w_{\text {base }} \cdot v_{c d 0} \cdot R_{p-D} \\
& a_{D 12,11}=-w_{\text {base }} \cdot w_{0} \\
& a_{D 13,2}= \frac{w_{\text {base }}\left[v_{b Q 0} \cos \left(\delta_{0}\right)-v_{b D 0} \operatorname{sen}\left(\delta_{0}\right)\right]}{-L_{c}} \\
& a_{D 13,11}=\frac{w_{\text {base }}}{L_{c}} \\
& a_{D 13,14}=w_{\text {base }} \cdot w_{0} \\
& a_{D 14,2}= \frac{\left[w_{\text {base }} v_{b D 0} \cos \left(\delta_{0}\right)+v_{b Q 0} \operatorname{sen}\left(\delta_{0}\right)\right]}{L_{c}} \\
& a_{D 14,12}=\frac{w_{\text {base }}}{L_{C}} \\
& a_{D 14,14}=-w_{\text {base }} \cdot \frac{R_{c}}{L_{c}} \\
& b 1_{D 13,2}=-w_{\text {base }} \cdot \frac{\operatorname{sen}\left(\delta_{0}\right)}{L_{c}} \\
& b 1_{D 14,2}=-w_{\text {base }} \cdot \cos \left(\delta_{0}\right) / L_{c}
\end{aligned}
$$

$$
a_{D 12,10}=\frac{w_{\text {base }}}{C_{f}}
$$$$
a_{D 12,14}=-\frac{w_{\text {base }}}{C_{f}}
$$$$
a_{D 13,3}=-w_{\text {base }} \cdot R_{p-D} \cdot i_{c q 0}
$$$$
a_{D 13,13}=-w_{\text {base }} \cdot \frac{R_{c}}{L_{c}}
$$$$
a_{D 14,3}=w_{\text {base }} \cdot R_{p-D} \cdot i_{c d 0}
$$$$
a_{D 14,13}=-w_{\text {base }} \cdot w_{0}
$$$$
b 1_{D 13,1}=-w_{\text {base }} \cdot \frac{\cos \left(\delta_{0}\right)}{L_{c}}
$$$$
b 1_{D 14,1}=w_{\text {base }} \cdot \frac{\operatorname{sen}\left(\delta_{0}\right)}{L_{c}}
$$$$
b 2_{D 1,2}=-w_{\text {base }}
$$

As equações relacionando as saídas do modelo (i.e., as correntes $i_{c D}$ e $i_{c Q}$ ) com as variáveis de estado são:

$$
\left[\Delta i_{C D Q}\right]=\left[C 1_{D}\right] \cdot\left[\Delta X_{D}\right]
$$

Onde,

$$
C 1_{D}=\left[\begin{array}{llllllllllllll}
0 & c 1_{D 1,2} & 0 & 0 & 0 & 0 & 0 & 0 & 0 & 0 & 0 & 0 & c 1_{D 1,13} & c 1_{D 1,14} \\
0 & c 1_{D 2,2} & 0 & 0 & 0 & 0 & 0 & 0 & 0 & 0 & 0 & 0 & c 1_{D 2,13} & c 1_{D 2,14}
\end{array}\right]
$$




$$
\begin{aligned}
c 1_{D 1,2} & =-i_{c q 0} \cdot \cos \left(\delta_{0}\right)-i_{c d 0} \cdot \operatorname{sen}\left(\delta_{0}\right) & c 1_{D 1,13} & =\cos \left(\delta_{0}\right) \\
c 1_{D 1,14} & =-\operatorname{sen}\left(\delta_{0}\right) & c 1_{D 2,2} & =i_{c d 0} \cdot \cos \left(\delta_{0}\right)-i_{c q 0} \cdot \operatorname{sen}\left(\delta_{0}\right) \\
c 1_{D 12,13} & =\operatorname{sen}\left(\delta_{0}\right) & c 1_{D 12,14} & =\cos \left(\delta_{0}\right)
\end{aligned}
$$

No caso da referência local d-q do Inversor com Controle Droop ser escolhida como a referência comum $D-Q$ do sistema, é necessário também expressar a velocidade em função das variáveis de estado através da seguinte expressão:

$$
\Delta w_{\text {com }}=\left[C 2_{D}\right] \cdot\left[\Delta X_{D}\right]
$$

Sendo,

$$
\begin{gathered}
C 2_{D}=\left[\begin{array}{llllllllllllll}
0 & 0 & c 2_{D 1,3} & 0 & 0 & 0 & 0 & 0 & 0 & 0 & 0 & 0 & 0 & 0
\end{array}\right] \\
c 2_{D 1,3}=-R_{p-D}
\end{gathered}
$$

\subsubsection{Controle Droop com Restauração de Frequência}

Com a implementação do Controle Droop, o equilíbrio entre a potência gerada e consumida ocorre em uma nova frequência de operação $\left(w=w^{*}+\Delta w\right)$. Sem a atuação de um controle secundário o sistema poderia continuar a operar em uma frequência diferente da programada (p.ex. $60 \mathrm{~Hz}$ ), prejudicando a operação de cargas sensíveis. O objetivo do Controle Droop com restaurador de Frequência $($ Controle Droop $+F)$ é precisamente restabelecer a frequência do sistema ao seu valor programado, emulando tanto o controle primário como o secundário dos grandes sistemas de potência. Basicamente sua estratégia de controle é similar ao Controle Droop (ver Figura 3.15), com a diferença que este controle apresenta mais uma malha de controle externa para restabelecer a frequência ao seu valor programado. Esta malha de controle é implementada no bloco denominado Gerenciador de Potência como mostrado na Figura 3.20 [23]. 


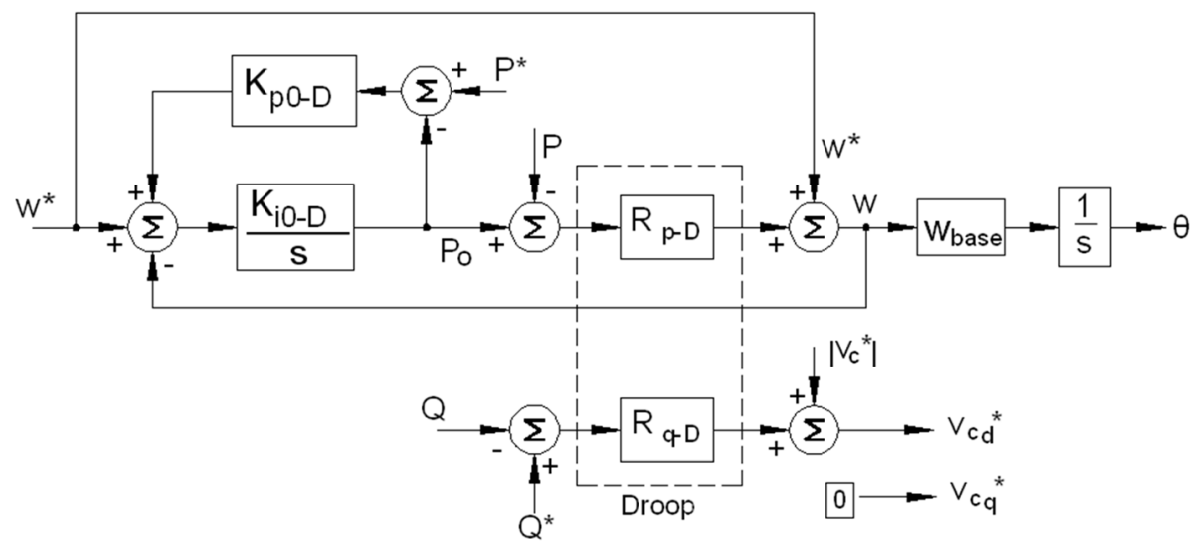

Figura 3.20 - Diagrama de blocos do Gerenciador de Potência - Controle Droop+F

Pode se observar que o único diferencial entre o Gerenciador de Potência do Controle Droop $+F$ e seu equivalente do Controle Droop se encontra na malha de controle que gera a referência $\theta$. As novas equações que descrevem o comportamento desta malha são:

$$
\begin{gathered}
\dot{P}_{o}=K_{p 0-D} \cdot K_{i 0-D} \cdot\left(P^{*}-P_{o}\right)+K_{i 0-D} \cdot\left(w^{*}-w\right) \\
w=w^{*}+\left(P_{o}-P\right) \cdot R_{p-D}
\end{gathered}
$$

O modelo completo no espaço de estados do inversor com Controle Droop $+F$ pode ser derivado diretamente a partir do modelo do Inversor com Controle Droop, incluindo a nova variável de estado $P_{o}$ e realizando as alterações necessárias relacionadas às eq.(3.85) e (3.86) $O$ modelo final é representado pela seguinte expressão:

$$
\left[\Delta \dot{X}_{D F}\right]=\left[A_{D F}\right] \cdot\left[\Delta X_{D F}\right]+\left[B 1_{D F}\right] \cdot\left[\Delta v_{b D Q}\right]+\left[B 2_{D F}\right] \cdot \Delta w_{c o m}
$$

Onde, 


$\left[A_{D F}\right]=\left[\begin{array}{ccccccccccccccc}0 & 0 & a_{D 1,3} & 0 & 0 & 0 & 0 & 0 & 0 & 0 & 0 & 0 & 0 & 0 & a_{D 1,15} \\ 0 & 0 & a_{D 2,3} & 0 & 0 & 0 & 0 & 0 & 0 & 0 & 0 & 0 & 0 & 0 & a_{D 2,15} \\ 0 & 0 & a_{D 3,3} & 0 & 0 & 0 & 0 & 0 & 0 & 0 & a_{D 3,11} & a_{D 3,12} & a_{D 3,13} & a_{D 3,14} & 0 \\ 0 & 0 & 0 & a_{D 4,4} & 0 & 0 & 0 & 0 & 0 & 0 & a_{D 4,11} & a_{D 4,12} & a_{D 4,13} & a_{D 4,14} & 0 \\ 0 & 0 & 0 & a_{D 5,4} & 0 & 0 & 0 & 0 & 0 & 0 & a_{D 5,11} & 0 & 0 & 0 & 0 \\ 0 & 0 & 0 & 0 & 0 & 0 & 0 & 0 & 0 & 0 & 0 & a_{D 6,12} & 0 & 0 & 0 \\ 0 & 0 & 0 & a_{D 7,4} & a_{D 7,5} & 0 & 0 & 0 & a_{D 7,9} & 0 & a_{D 7,11} & a_{D 7,12} & a_{D 7,13} & 0 & 0 \\ 0 & 0 & 0 & 0 & 0 & a_{D 8,6} & 0 & 0 & 0 & a_{D 8,10} & a_{D 8,11} & a_{D 8,12} & 0 & a_{D 8,14} & 0 \\ 0 & 0 & a_{D 9,3} & a_{D 9,4} & a_{D 9,5} & 0 & a_{D 9,7} & 0 & a_{D 9,9} & a_{D 9,10} & a_{D 9,11} & a_{D 9,12} & a_{D 9,13} & 0 & a_{D 9,15} \\ 0 & 0 & a_{D 10,3} & 0 & 0 & a_{D 10,6} & 0 & a_{D 10,8} & a_{D 10,9} & a_{D 10,10} & a_{D 10,11} & a_{D 10,12} & 0 & a_{D 10,14} & a_{D 10,15} \\ 0 & 0 & a_{D 11,3} & 0 & 0 & 0 & 0 & 0 & a_{D 11,9} & 0 & 0 & a_{D 11,12} & a_{D 11,13} & 0 & a_{D 11,15} \\ 0 & 0 & a_{D 12,3} & 0 & 0 & 0 & 0 & 0 & 0 & a_{D 12,10} & a_{D 12,11} & 0 & 0 & a_{D 12,14} & a_{D 12,15} \\ 0 & a_{D 13,2} & a_{D 13,3} & 0 & 0 & 0 & 0 & 0 & 0 & 0 & a_{D 13,11} & 0 & a_{D 13,13} & a_{D 13,14} & a_{D 13,15} \\ 0 & a_{D 14,2} & a_{D 14,3} & 0 & 0 & 0 & 0 & 0 & 0 & 0 & 0 & a_{D 14,12} & a_{D 14,13} & a_{D 14,14} & a_{D 14,15} \\ 0 & 0 & a_{D 15,3} & 0 & 0 & 0 & 0 & 0 & 0 & 0 & 0 & 0 & 0 & 0 & a_{D 15,15}\end{array}\right]$




$$
\begin{aligned}
& {\left[\Delta X_{D F}\right]=\left[\begin{array}{c}
\Delta \theta \\
\Delta \delta \\
\Delta P \\
\Delta Q \\
\Delta \emptyset_{D-d} \\
\Delta \emptyset_{D-q} \\
\Delta \psi_{D-d} \\
\Delta \psi_{D-q} \\
\Delta i_{f d} \\
\Delta i_{f q} \\
\Delta v_{c d} \\
\Delta v_{c q} \\
\Delta i_{c d} \\
\Delta i_{c q} \\
\Delta P_{o}
\end{array}\right] \quad\left[B 1_{D F}\right]=\left[\begin{array}{cc}
0 & 0 \\
0 & 0 \\
0 & 0 \\
0 & 0 \\
0 & 0 \\
0 & 0 \\
0 & 0 \\
0 & 0 \\
0 & 0 \\
0 & 0 \\
0 & 0 \\
0 & 0 \\
b 1_{D 13,1} & b 1_{D 13,2} \\
b 1_{D 14,1} & b 1_{D 14,2} \\
0 & 0
\end{array}\right] \quad B 2_{D F}=\left[\begin{array}{c}
0 \\
b 2_{D 1,2} \\
0 \\
0 \\
0 \\
0 \\
0 \\
0 \\
0 \\
0 \\
0 \\
0 \\
0 \\
0 \\
0
\end{array}\right]} \\
& a_{D 1,15}=w_{\text {base }} \cdot R_{p-D} \\
& a_{D 2,15}=w_{\text {base }} \cdot R_{p-D} \\
& a_{D 9,15}=w_{\text {base }} \cdot R_{p-D} \cdot i_{f q 0} \\
& a_{D 10,15}=-w_{\text {base }} \cdot R_{p-D} \cdot i_{f d 0} \\
& a_{D 11,15}=w_{\text {base }} \cdot R_{p-D} \cdot v_{c q 0} \\
& a_{D 12,15}=-w_{\text {base }} \cdot R_{p-D} \cdot v_{c d 0} \\
& a_{D 13,15}=w_{\text {base }} \cdot R_{p-D} \cdot i_{c q 0} \\
& a_{D 14,15}=-w_{\text {base }} \cdot R_{p-D} \cdot i_{c d 0} \\
& a_{D 15,3}=K_{i 0-D} \cdot R_{p-D} \\
& a_{D 15,15}=-K_{i 0-D} \cdot\left(K_{p 0-D}+R_{p-D}\right)
\end{aligned}
$$

As equações relacionando as saídas do modelo (i.e., as correntes $i_{c D}$ e $i_{c Q}$ ) com as variáveis de estado são:

$$
\left[\Delta i_{C D Q}\right]=\left[C 1_{D F}\right] \cdot\left[\Delta X_{D F}\right]
$$

Onde,

$$
C 1_{D F}=\left[\begin{array}{lllllllllllllll}
0 & c 1_{D 1,2} & 0 & 0 & 0 & 0 & 0 & 0 & 0 & 0 & 0 & 0 & c 1_{D 1,13} & c 1_{D 1,14} & 0 \\
0 & c 1_{D 2,2} & 0 & 0 & 0 & 0 & 0 & 0 & 0 & 0 & 0 & 0 & c 1_{D 2,13} & c 1_{D 2,14} & 0
\end{array}\right]
$$

No caso da referência local $d-q$ do Inversor com Controle Droop $F$ ser escolhida como a referência comum $D-Q$ do sistema, é necessário também expressar a velocidade em função das variáveis de estado através da seguinte expressão: 


$$
\Delta w_{\text {com }}=\left[C 2_{D F}\right] \cdot\left[\Delta X_{D F}\right]
$$

Sendo,

$$
\begin{aligned}
C 2_{D F}=\left[\begin{array}{ccccccccccccccc}
0 & 0 & c 2_{D 1,3} & 0 & 0 & 0 & 0 & 0 & 0 & 0 & 0 & 0 & 0 & 0 & c 2_{D 1,15}
\end{array}\right] \\
c 2_{D 1,15}=R_{p-D}
\end{aligned}
$$

\subsection{Modelagem das Cargas}

\subsubsection{Motor de Indução}

As equações diferenciais que descrevem o comportamento dinâmico de um motor de indução trifásico tipo gaiola de esquilo são desenvolvidas em detalhe em [29] e [34]. Aqui unicamente se mostra a manipulação destas equações de tal forma a se desenvolver um modelo linear no espaço de estados da máquina, compatível com o modelo padrão mostrado na Figura 3.2. O modelo utilizado para representar o motor de indução se baseia na utilização de bobinas fictícias representadas sobre uma referência rotativa de eixo direto $(d)$ e quadratura $(q)$ que gira em velocidade síncrona $\left(w_{s}\right)$ como se mostra na Figura 3.21. Os enrolamentos reais do estator e do rotor são representados por duas bobinas fictícias cada, dispostas sobre os eixos $d$ e $q$ com indutâncias próprias $L_{s s}$ e $L_{r r}$ respectivamente. As equações que modelam o comportamento dinâmico do motor de indução em pu são:

Equações de tensão do estator:

$$
e_{s d}=\frac{1}{w_{\text {base }}} \cdot \dot{\psi_{s d}}-\psi_{s q} \cdot w_{s}+R_{s} \cdot i_{s d}
$$




$$
e_{s q}=\frac{1}{w_{\text {base }}} \cdot \dot{\psi_{s q}}+\psi_{s d} \cdot w_{s}+R_{s} \cdot i_{s q}
$$

Equações de tensão do rotor:

$$
\begin{aligned}
& 0=\frac{1}{w_{\text {base }}} \cdot \dot{\psi_{r d}}-\left(w_{s}-w_{r}\right) \cdot \psi_{r q}+R_{r} \cdot i_{r d} \\
& 0=\frac{1}{w_{\text {base }}} \cdot \dot{\psi_{r q}}+\left(w_{s}-w_{r}\right) \cdot \psi_{r d}+R_{r} \cdot i_{r q}
\end{aligned}
$$

Equações de enlaces de fluxo do estator:

$$
\begin{aligned}
& \psi_{s d}=L_{s s} \cdot i_{s d}+L_{m} \cdot i_{r d} \\
& \psi_{s q}=L_{s s} \cdot i_{s q}+L_{m} \cdot i_{r q}
\end{aligned}
$$

Equações de enlaces de fluxo do rotor:

$$
\begin{aligned}
& \psi_{r d}=L_{r r} \cdot i_{r d}+L_{m} \cdot i_{s d} \\
& \psi_{r q}=L_{r r} \cdot i_{r q}+L_{m} \cdot i_{s q}
\end{aligned}
$$

Equação do torque e equação mecânica:

$$
\begin{gathered}
T_{e}=\psi_{s d} \cdot i_{s q}-\psi_{s q} \cdot i_{s d} \\
\dot{w}_{r}=\frac{1}{2 H_{A S}} \cdot\left(T_{e}-T_{m}-K_{A S D} \cdot w_{r}\right)
\end{gathered}
$$




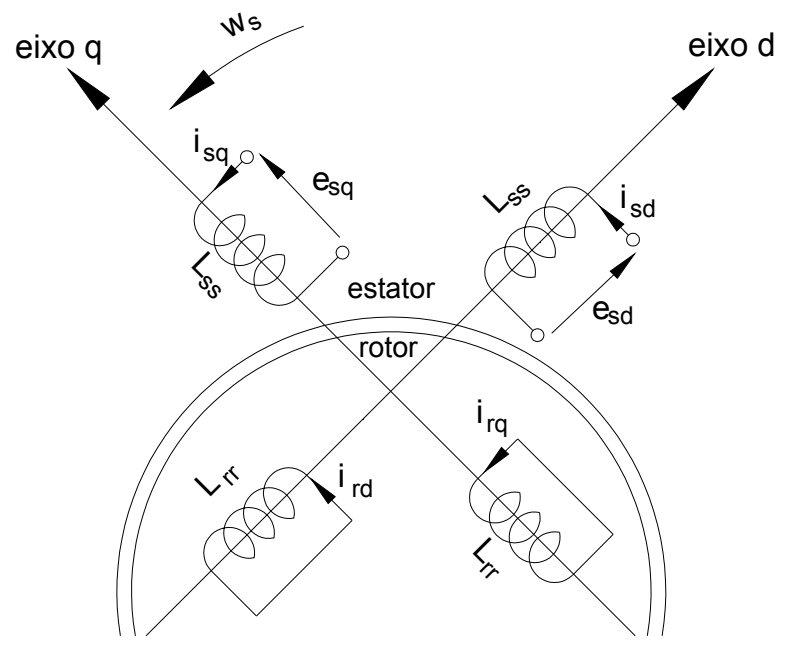

Figura 3.21 - Circuitos equivalentes do estator e rotor de um motor de indução

Onde,

$e_{k}, i_{k}, \psi_{k} \quad=$ tensão, corrente e enlaces de fluxo do enrolamento " $k$ " $(\operatorname{com} k=s d, s q, r d, r q)$.

$R_{S} \quad=$ resistência de armadura por fase.

$R_{r} \quad=$ resistência do rotor referida ao estator.

$L_{S S} \quad=$ indutância própria do enrolamento de estator.

$L_{r r} \quad=$ indutância própria do rotor referida ao estator.

$L_{m} \quad=$ indutância mútua estator-rotor.

$T_{e}$ e $T_{m} \quad$ = torque eletromagnético e torque mecânico.

$K_{A S D} \quad=$ coeficiente de atrito.

$w_{S} \quad=$ velocidade angular elétrica síncrona.

$w_{r} \quad=$ velocidade angular elétrica do rotor.

$w_{\text {base }} \quad=$ valor de base da velocidade angular elétrica $[\mathrm{rad} / \mathrm{s}]$.

$H_{A S} \quad=$ constante de inércia [s].

As equações (3.90) à (3.99) descrevem a dinâmica do motor de indução com $w_{r}, \psi_{s d}, \psi_{s q}, \psi_{r d}$ e $\psi_{r q}$ como variáveis de estado. Porém, as derivadas destas variáveis aparecem nestas equações como função de $i_{s d}, i_{s q}, i_{r d}, i_{r q} e T_{e}$, que não são nem variáveis de estado nem entradas. Consequentemente, é necessário 
expressar as correntes dos enrolamentos e o torque eletromagnético em termos das variáveis de estado através da seguinte expressão:

$$
\left[\begin{array}{l}
i_{s d} \\
i_{s q} \\
i_{r d} \\
i_{r q}
\end{array}\right]=\left[\begin{array}{cccc}
A_{A S 1,1} & 0 & A_{A S 1,3} & 0 \\
0 & A_{A S 2,2} & 0 & A_{A S 2,4} \\
A_{A S 3,1} & 0 & A_{A S 3,3} & 0 \\
0 & A_{A S 4,2} & 0 & A_{A S 4,4}
\end{array}\right] \cdot\left[\begin{array}{l}
\psi_{s d} \\
\psi_{s q} \\
\psi_{r d} \\
\psi_{r q}
\end{array}\right]
$$

Onde,

$$
\begin{array}{ll}
A_{A S 1,1}=A_{A S 2,2}=-\frac{L_{r r}}{L_{m}{ }^{2}-L_{S S} \cdot L_{r r}} & A_{A S 1,3}=A_{A S 2,4}=\frac{L_{m}}{L_{m}{ }^{2}-L_{s S} \cdot L_{r r}} \\
A_{A S 3,1}=A_{A S 4,2}=\frac{L_{m}}{L_{m}{ }^{2}-L_{s s} \cdot L_{r r}} & A_{A S 3,3}=A_{A S 4,4}=-\frac{L_{S S}}{L_{m}{ }^{2}-L_{s S} \cdot L_{r r}}
\end{array}
$$

Substituindo as expressões para as correntes da eq. (3.100) na eq.(3.98), e linearizando a expressão resultante por expansão em série de Taylor, permite obter a equação linearizada do torque eletromagnético em função unicamente das variáveis de estado.

$$
\Delta T_{e}=K_{A S 1 .} \Delta \psi_{s d}+K_{A S 2 .} \Delta \psi_{s q}+K_{A S 3 .} \Delta \psi_{r d}+K_{A S 4 .} \Delta \psi_{r q}
$$

Sendo,

$$
\begin{array}{ll}
K_{A S 1}=L_{m} \cdot \frac{\psi_{r q 0}}{L_{m}^{2}-L_{r r} \cdot L_{s s}} & K_{A S 2}=-L_{m} \cdot \frac{\psi_{r d 0}}{L_{m}{ }^{2}-L_{r r} \cdot L_{s s}} \\
K_{A S 3}=-L_{m} \cdot \frac{\psi_{s q 0}}{L_{m}{ }^{2}-L_{r r} \cdot L_{s s}} & K_{A S 4}=L_{m} \cdot \frac{\psi_{s d 0}}{L_{m}{ }^{2}-L_{r r} \cdot L_{s s}}
\end{array}
$$

Similarmente, substituindo as expressões para as correntes da eq.(3.100), nas eq. (3.90) à (3.93), e linearizando a expressão resultante por expansão em série de Taylor, permite obter o modelo completo no espaço de estados do Motor de Indução mostrado a seguir: 


$$
\left[\Delta \dot{X}_{A S}\right]=\left[A_{A S}\right] \cdot\left[\Delta X_{A S}\right]+\left[B 1_{A S}\right] \cdot\left[\Delta v_{b D Q}\right]+\left[B 2_{A S}\right] \cdot \Delta w_{c o m}
$$

Onde,

$$
\begin{aligned}
& {\left[A_{A S}\right]=\left[\begin{array}{ccccc}
a_{A S 1,1} & a_{A S 1,2} & a_{A S 1,3} & a_{A S 1,4} & a_{A S 1,5} \\
0 & a_{A S 2,2} & a_{A S 2,3} & a_{A S 2,4} & 0 \\
0 & a_{A S 3,2} & a_{A S 3,3} & 0 & a_{A S 3,5} \\
a_{A S 4,1} & a_{A S 4,2} & 0 & a_{A S 4,4} & a_{A S 4,5} \\
a_{A S 5,1} & 0 & a_{A S 5,3} & a_{A S 5,4} & a_{A S 5,5}
\end{array}\right]} \\
& {\left[\Delta X_{A S}\right]=\left[\begin{array}{c}
\Delta w_{r} \\
\Delta \psi_{s d} \\
\Delta \psi_{s q} \\
\Delta \psi_{r d} \\
\Delta \psi_{r q}
\end{array}\right] \quad B 1_{A S}=\left[\begin{array}{cc}
0 & 0 \\
b 1_{A S 2,1} & b 1_{A S 2,2} \\
b 1_{A S 3,1} & b 1_{A S 3,2} \\
0 & 0 \\
0 & 0
\end{array}\right] \quad B 2_{A S}=\left[\begin{array}{c}
0 \\
b 2_{A S 2,1} \\
b 2_{A S 3,1} \\
b 2_{A S 4,1} \\
b 2_{A S 5,1}
\end{array}\right]} \\
& a_{A S 1,1}=-\frac{K_{A S D}}{2 \cdot H_{A S}} \quad a_{A S 1,2}=\frac{K_{A S 1}}{2 \cdot H_{A S}} \quad a_{A S 1,3}=\frac{K_{A S 2}}{2 \cdot H_{A S}} \\
& a_{A S 1,4}=\frac{K_{A S 3}}{2 \cdot H_{A S}} \quad a_{A S 1,5}=\frac{K_{A S 4}}{2 \cdot H_{A S}} \quad \begin{array}{ll}
a_{A S 2,2}=-w_{\text {base }} \cdot R_{s} \cdot A_{A S 1,1} \\
a_{A S 3,2}=-w_{\text {base }} \cdot w_{S 0}
\end{array} \\
& a_{A S 2,3}=w_{\text {base }} \cdot w_{S 0} \quad a_{A S 2,4}=-w_{\text {base }} \cdot R_{S} \cdot A_{A S 1,3} \quad a_{A S 4,1}=-w_{\text {base }} \cdot \psi_{\text {rq0 }} \\
& a_{A S 3,3}=-w_{\text {base }} \cdot R_{s} \cdot A_{A S 2,2} \quad a_{A S 3,5}=-w_{\text {base }} \cdot R_{s} \cdot A_{A S 2,4} \quad a_{A S 4,5}=w_{\text {base }} \cdot\left(w_{S 0}-w_{r 0}\right) \\
& a_{A S 4,2}=-w_{\text {base }} \cdot R_{r} \cdot A_{A S 3,1} \quad a_{A S 4,4}=-w_{\text {base }} \cdot R_{r} \cdot A_{A S 3,3} \quad a_{A S 5,4} w_{\text {base }} \cdot\left(w_{r 0}-w_{S 0}\right) \\
& a_{A S 5,1}=w_{\text {base }} \cdot \psi_{\text {rd } 0} \quad a_{A S 5,3}=-w_{\text {base }} \cdot R_{r} \cdot A_{A S 4,2} \quad b 1_{A S 2,2}=w_{\text {base }} \cdot \operatorname{sen}\left(\delta_{0}\right) \\
& a_{A S 5,5}=-w_{\text {base }} \cdot R_{r} \cdot A_{A S 4,4} \quad b 1_{A S 2,1}=w_{\text {base }} \cdot \cos \left(\delta_{0}\right) \quad b 1_{A S 3,1}=-w_{\text {base }} \cdot \operatorname{sen}\left(\delta_{0}\right) \\
& b 1_{A S 3,2}=w_{\text {base }} \cdot \cos \left(\delta_{0}\right) \quad b 2_{A S 2,1}=w_{\text {base }} \cdot \psi_{s q 0} \quad b 2_{A S 3,1}=-w_{\text {base }} \cdot \psi_{s d 0} \\
& b 2_{A S 4,1}=w_{\text {base }} \cdot \psi_{\text {rq0 }} \quad b 2_{A S 5,1}=-w_{\text {base }} \cdot \psi_{r d 0}
\end{aligned}
$$

As equações relacionando as saídas do modelo (i.e., as correntes $i_{S D}$ e $i_{s Q}$ ) com as variáveis de estado são: 


$$
\left[\Delta i_{S D Q}\right]=\left[C 1_{A S}\right] \cdot\left[\Delta X_{A S}\right]
$$

Onde,

$$
\begin{array}{r}
C 1_{A S}=\left[\begin{array}{ccccc}
0 & c 1_{A S 1,2} & 0 & c 1_{A S 1,4} & 0 \\
0 & 0 & c 1_{A S 2,3} & 0 & c 1_{A S 2,5}
\end{array}\right] \\
c 1_{A S 1,2}=-A_{A S 1,1} \\
c 1_{A S 2,3}=-A_{A S 2,2}
\end{array}
$$

\subsubsection{Cargas Passivas de Impedância Constante (R-L)}

As equações diferenciais que descrevem o comportamento de uma carga trifásica de impedância constante (R-L) ligada na rede podem ser derivadas aplicando-se a segunda lei de Kirchhoff ao circuito mostrado na Figura 3.22. Em termos das componentes $D-Q$, estas equações em pu são:

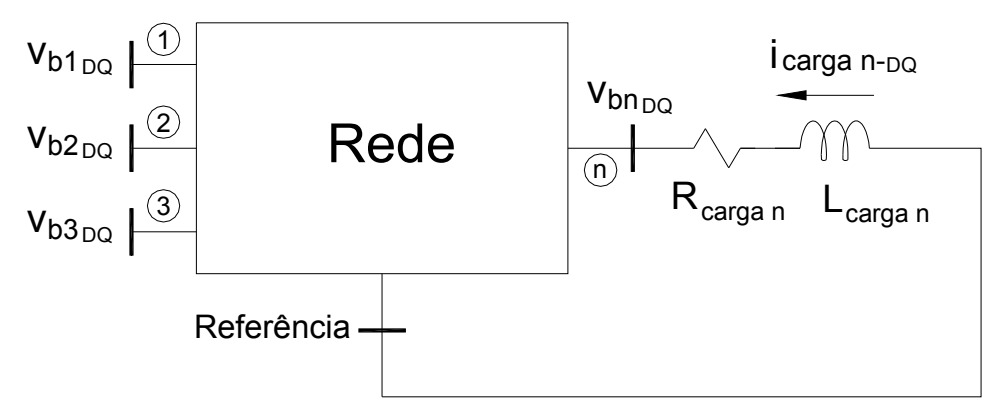

Figura 3.22 - Conexão de Cargas de Impedância Constante R-L

$$
\underset{\mathrm{i}_{\text {carga } \mathrm{n}-\mathrm{D}}}{\cdot}=\frac{w_{\text {base }}}{L_{\text {cargan }}} \cdot\left(-R_{\text {carga } n} \cdot i_{\text {carga } n-D}+w \cdot L_{\text {carga }} \cdot i_{\text {carga } n-Q}-v_{b n-D}\right)
$$




$$
\mathrm{i}_{\text {carga } \mathrm{n}-\mathrm{Q}}=\frac{w_{\text {base }}}{L_{\text {carga } n}} \cdot\left(-R_{\text {carga } n} \cdot i_{\text {carga } n-Q}-w \cdot L_{\text {carga }} \cdot i_{\text {carga } n-D}-v_{b n-Q}\right)
$$

Onde,

$$
\begin{array}{ll}
R_{\text {carga } n} e L_{\text {cargan }} & =\text { resistência e indutância da } n \text {-ésima carga } . \\
v_{b n} & =\text { tensão do } n \text {-ésimo nó da rede. } \\
w & =\text { frequência da rede }[\mathrm{rad} / \mathrm{seg}]
\end{array}
$$

Linearizando a eq.(3.104) e eq. (3.105) por expansão em série de Taylor, se tem o modelo completo no espaço de estados da $n$-ésima carga, mostrado a seguir.

$$
\left[\Delta X_{\operatorname{cargan} n}\right]=\left[A_{\operatorname{cargan} n}\right]\left[\Delta X_{\operatorname{cargan} n}\right]+\left[B 1_{\operatorname{carga} n}\right]\left[\Delta v_{b n-D Q}\right]+\left[B 2_{\operatorname{carga} n}\right] \cdot \Delta w_{\operatorname{com}}(3.106)
$$

Sendo,

$$
\begin{aligned}
& A_{\text {carga } n}=\left[\begin{array}{ll}
a_{\text {carga } 1,1} & a_{\text {carga } 1,2} \\
a_{\text {carga } 2,1} & a_{\text {carga } 2,2}
\end{array}\right] \\
& B 1_{\text {cargan }}=\left[\begin{array}{cc}
b 1_{\text {carga } 1,1} & 0 \\
0 & b 1_{\text {carga } 2,2}
\end{array}\right] \\
& B 2_{\operatorname{carga} n}=\left[\begin{array}{l}
b 2_{\text {carga } 1,1} \\
b 2_{\text {carga } 2,1}
\end{array}\right] \\
& {\left[\Delta X_{\text {cargan }}\right]=\left[\begin{array}{l}
\Delta i_{\text {carga } n-D} \\
\Delta i_{\text {carga } n-Q}
\end{array}\right]} \\
& {\left[\Delta v_{b n-D Q}\right]=\left[\begin{array}{l}
\Delta v_{b n-D} \\
\Delta v_{b n-Q}
\end{array}\right]} \\
& a_{\text {carga } 1,1}=-w_{\text {base }} \cdot \frac{R_{\text {carga } n}}{L_{\text {carga } n}} \\
& a_{\text {carga } 1,2}=w_{\text {base }} \cdot w_{0} \\
& a_{\text {carga } 2,1}=-a_{\text {carga } 1,2} \\
& a_{\text {carga } 2,2}=a_{\text {carga } 1,1}
\end{aligned}
$$




$$
\begin{aligned}
b 1_{\text {carga } 1,1} & =\frac{w_{\text {base }}}{L_{\text {carga } n}} \\
b 2_{\text {carga } 1,1} & =w_{\text {base }} \cdot i_{\text {carga } n-Q 0}
\end{aligned}
$$

$$
\begin{aligned}
& b 2_{\text {carga } 2,2}=b 1_{\text {carga } 1,1} \\
& b 2_{\text {carga } 2,1}=-w_{\text {base }} \cdot i_{\text {carga } n-D 0}
\end{aligned}
$$

As equações relacionando as saídas do modelo (i.e., as correntes $i_{\text {carga } n-D} \mathrm{e}$ $i_{\text {carga } n-Q}$ ) com as variáveis de estado são:

$$
\left[\Delta i_{\operatorname{cargan}-D Q}\right]=\left[C 1_{\operatorname{cargan} n}\right] \cdot\left[\Delta X_{\operatorname{cargan} n}\right]
$$

Onde,

$$
\begin{gathered}
{\left[C 1_{\text {carga } n}\right]=\left[\begin{array}{cc}
c 1_{\text {carga } 1,1} & 0 \\
0 & c 1_{\text {carga } 2,2}
\end{array}\right]} \\
c 1_{\text {carga } 1,1}=c 1_{\text {carga } 2,2}=1
\end{gathered}
$$

\subsection{Modelagem da Rede}

Como explicado na Seção 3.1.1, os transitórios de alta frequência resultantes da interação entre os inversores com os outros componentes da MG gerou a necessidade de considerar os transitórios eletromagnéticos do estator da máquina síncrona (assim como também do motor de indução) no desenvolvimento dos respectivos modelos dinâmicos.

Consequentemente, para garantir a compatibilidade entre o modelo da Rede e os modelos das máquinas é necessário desenvolver um modelo de Rede baseado em equações diferenciais, e não apenas em equações fasoriais (algébricas) como é prática comum na análise de estabilidade dinâmica de grandes sistemas de potência. 
O comprimento dos alimentadores das MGs usualmente está na faixa de dezenas a centenas de metros. Assim, pode se utilizar um modelo de parâmetros concentrados R-L em série para representar a Rede, negligenciando-se a capacitância shunt [51]. A Figura 3.23 mostra uma representação da Rede.

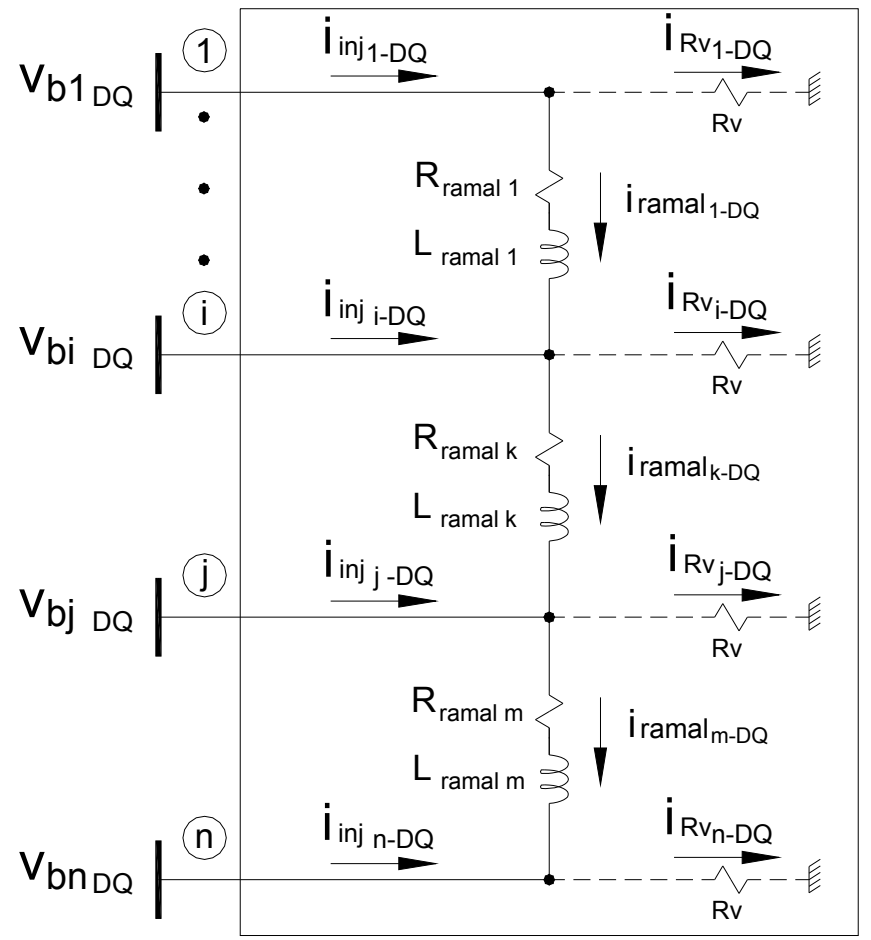

Figura 3.23 - Representação da Rede

As equações diferenciais que descrevem o comportamento de cada ramal da Rede (trecho compreendido entre dois nós) podem ser derivadas aplicando-se a segunda lei de Kirchhoff ao circuito da Figura 3.23. Em termos das componentes D-Q, estas equações em pu são (para o ramal genérico $m$ ):

$$
\begin{aligned}
\mathrm{i}_{\text {ramal } \mathrm{m}-\mathrm{D}}= & \frac{w_{\text {base }}}{L_{\text {ramal } m}}\left(-R_{\text {ramal } m} \cdot i_{\text {ramal } m-D}+w \cdot L_{\text {ramal m }} \cdot i_{\text {ramal } m-Q}+v_{b j-D}\right. \\
& \left.-v_{\text {bn-D }}\right)
\end{aligned}
$$




$$
\begin{aligned}
\mathrm{i}_{\text {ramal } \mathrm{m}-\mathrm{Q}}= & \frac{w_{\text {base }}}{L_{\text {ramal } m}}\left(-R_{\text {ramal } m} \cdot i_{\text {ramal } m-Q}-w \cdot L_{\text {ramal } m} \cdot i_{\text {ramal } m-D}+v_{b j-Q}\right. \\
& \left.-v_{\text {bn-Q }}\right)
\end{aligned}
$$

Onde,

$$
\begin{array}{ll}
R_{\text {ramal m }} \text { e } L_{\text {ramal m }} & =\text { resistência e indutância do } m \text {-ésimo ramal. } \\
v_{b j} \text { e } v_{b n} & =\text { tensão do j-ésimo e } n \text {-ésimo nó da rede. } \\
w & =\text { frequência da rede }[\mathrm{rad} / \mathrm{seg}]
\end{array}
$$

Para acoplar os modelos no espaço de estados, das fontes e cargas com o modelo da rede, é necessário derivar uma equação que relacione as tensões dos nós com as correntes injetadas pelas fontes e as cargas na rede (i.e., as saídas dos modelos no espaço de estados). Para isto, faz se uso de um artificio que consiste em utilizar um resistor virtual $\left(R_{v}\right)$ de grande magnitude $(\geq 1000 \Omega)$ conectado entre cada nó da rede e a terra, como mostrado na Figura 3.23.

Com este artificio, a tensão do nó genérico $j$ fica definida pela seguinte expressão:

$$
v_{b j}=R_{v} \cdot i_{R v j}
$$

Aplicando-se a primeira lei de Kirchhoff ao nó $j$ da rede se tem:

$$
i_{R v j}=i_{\text {inj } j}+i_{\text {ramal } k}-i_{\text {ramal } m}
$$

Onde $i_{i n j j}$ é a corrente injetada pela fonte ou carga no j-ésimo nó 
A substituição da eq. (3.112) na eq. (3.111) permite obter a relação desejada entre tensões de nós e correntes injetadas na rede:

$$
v_{b j}=R_{v} \cdot\left(i_{\text {inj } j}+i_{\text {ramal } k}-i_{\text {ramal } m}\right)
$$

O modelo linearizado da Rede se obtém ao linearizar a eq. (3.109), eq. (3.110) e eq. (3.113) por expansão em série de Taylor e se mostra a seguir para o $m$-ésimo ramal genérico da rede na referência comum $D-Q$ :

$$
\begin{aligned}
& {\left[\Delta X_{\text {ramal m }}\right]=\left[A_{\text {ramal m }}\right] \cdot\left[\Delta X_{\text {ramal m }}\right]+\left[B 1_{\text {ramal m }}\right] \cdot\left[\Delta v_{b j-D Q}\right] } \\
&+\left[B 2_{\text {ramal m }}\right] \cdot\left[\Delta v_{b n-D Q}\right]+\left[B 3_{\text {ramal }}\right] \cdot \Delta w_{\text {com }}
\end{aligned}
$$

Onde,

$$
\begin{aligned}
& {\left[A_{\text {ramal }}\right]=\left[\begin{array}{ll}
a_{\text {ramal } 1,1} & a_{\text {ramal } 1,2} \\
a_{\text {ramal } 2,1} & a_{\text {ramal } 2,2}
\end{array}\right] \quad\left[B 1_{\text {ramal } m}\right]=\left[\begin{array}{cc}
b 1_{\text {ramal } 1,1} & 0 \\
0 & b 1_{\text {ramal } 2,2}
\end{array}\right]} \\
& {\left[B 2_{\text {ramal }}\right]=\left[\begin{array}{cc}
b 2_{\text {ramal1,1 }} & 0 \\
0 & b 2_{\text {ramal } 2,2}
\end{array}\right] \quad\left[B 3_{\text {ramal m }}\right]=\left[\begin{array}{l}
b 3_{\text {ramal } 1,1} \\
b 3_{\text {ramal }, 1}
\end{array}\right]} \\
& {\left[\Delta X_{\text {ramal } m}\right]=\left[\begin{array}{l}
\Delta i_{\text {ramal } m-D} \\
\Delta i_{\text {ramal } m-Q}
\end{array}\right] \quad\left[\Delta v_{b j-D Q}\right]=\left[\begin{array}{l}
\Delta v_{b j-D} \\
\Delta v_{b j-Q}
\end{array}\right] \quad \Delta v_{b n-D Q}=\left[\begin{array}{l}
\Delta v_{b n-D} \\
\Delta v_{b n-Q}
\end{array}\right]} \\
& \begin{array}{ll}
a_{\text {ramal } 1,1}=-w_{\text {base }} \cdot \frac{R_{\text {ramal } m}}{L_{\text {ramal } m}} & a_{\text {ramal } 1,2}=w_{\text {base }} \cdot w_{0} \\
& a_{\text {ramal } 2,2}=a_{\text {ramal } 1,1}
\end{array} \\
& a_{\text {ramal } 2,1}=-a_{\text {ramal } 1,2} \\
& b 1_{\text {ramal } 1,1}=\frac{w_{\text {base }}}{L_{\text {ramal } m}} \quad b 1_{\text {ramal } 2,2}=b 1_{\text {ramal } 1,1} \\
& b 2_{\text {ramal1,1 }}=-b 1_{\text {ramal } 1,1} \quad b 2_{\text {ramal } 2,2}=-b 1_{\text {rama1,1 }} \\
& b 3_{\text {ramal1,1 }}=w_{\text {base }} \cdot i_{\text {ramal } m-Q 0} \\
& b 3_{\text {ramal } 2,1}=-w_{\text {base }} \cdot i_{\text {ramal } m-D 0}
\end{aligned}
$$


As equações linearizadas relacionando as tensões de nó com as correntes injetadas na rede são (para o nó genérico $j$ ):

$$
\begin{gathered}
{\left[\Delta v_{b j-D Q}\right]=\left[C 1_{\text {nó-j }}\right] \cdot\left[\Delta i_{\text {inj } j-D Q}\right]+\left[C 2_{\text {ramal m }}\right] \cdot\left[\Delta i_{\text {ramal m }}\right]} \\
+\left[C 3_{\text {ramal }}\right] \cdot\left[\Delta i_{\text {ramal } k}\right]
\end{gathered}
$$

Sendo,

$$
\begin{aligned}
& {\left[C 1_{n o ́-j}\right]=\left[\begin{array}{cc}
c 1_{\text {nó } 1,1} & 0 \\
0 & c 1_{\text {nó } 2,2}
\end{array}\right] \quad\left[C 2_{\text {ramal m }}\right]=\left[\begin{array}{cc}
c 2_{\text {ramal1,1 }} & 0 \\
0 & c 2_{\text {ramal } 2,2}
\end{array}\right]} \\
& {\left[C 3_{\text {ramal k }}\right]=\left[\begin{array}{cc}
c 3_{\text {ramal } 1,1} & 0 \\
0 & c 3_{\text {ramal }, 2}
\end{array}\right]} \\
& c 1_{n o ́ 1,1}=R_{v} \\
& c 1_{\text {nó } 2,2}=c 1_{\text {nó1,1 }} \\
& c 2_{\text {ramal1,1}}=-c 1_{\text {nó1,1 }} \\
& c 2_{\text {ramal2,2 }}=-c 1_{\text {nó1,1 }} \\
& c 3_{\text {ramal1,1 }}=c 1_{\text {nó } 1,1} \\
& c 3_{\text {ramal2,2 }}=c 1_{\text {nó1,1 }}
\end{aligned}
$$




\section{Análise da Operação Individual das FEDs}

Como mencionado no Capítulo 1, um dos elementos chaves para o desenvolvimento modular de uma MG é a característica "ligar e usar" que o UCM deve proporcionar a sua respectiva FED. Esta característica deve garantir que uma FED comercial, com os ajustes padrões de fábrica, possa ser adicionada a uma MG sem a necessidade de recalibrar, ou até mesmo reconfigurar, as malhas de controle das FEDs existentes.

Com o objetivo de analisar as implicações desta característica do ponto de vista da estabilidade dinâmica, neste capítulo se realiza o ajuste individual das malhas de controle das FEDs modeladas no Capítulo 3. Para simular o ajuste padrão de fábrica, as FEDs (ou os inversores associadas a estas) são calibradas de forma a atender às especificações da resposta transitória segundo Normas. Os valores adequados dos ganhos dos controladores assim obtidos (sem ajustes posteriores) serão utilizados para analisar a estabilidade dinâmica de combinações de FEDs no Capítulo 5, avaliando assim as implicações da característica "ligar e usar" na estabilidade da MG.

Visto que os ajustes dos parâmetros das malhas de controle dependem da capacidade dos microgeradores, é necessário definir as potências nominais das FEDs a serem utilizadas na análise. Para fazer o estudo mais abrangente, dois valores de potência nominal serão analisados para cada tipo de FED. As potências escolhidas serão aquelas comumente encontradas em FEDs comerciais, adequadas para utilização em redes de BT trifásicas de $220 \mathrm{~V}$.

\subsection{Grupo Gerador Diesel}

\subsubsection{Ajuste das malhas de controle}

Quando uma carga elétrica é conectada (ou desconectada) subitamente nos terminais de um GGD, a frequência (velocidade da máquina) e a tensão são 
temporariamente alteradas da sua condição de regime. Esta mudança é conhecida como resposta transitória. Frequentemente as características de desempenho de um sistema de controle são especificadas em termos da resposta transitória para uma entrada degrau unitário, pois esta entrada é fácil de gerar e corresponde a uma solicitação suficientemente severa. Assim sendo, para o ajuste das malhas de controle é de interesse conhecer as especificações no domínio do tempo que as respostas transitórias dos reguladores de tensão e velocidade devem apresentar de forma a atender os requisitos básicos de desempenho especificados pelas normas nacionais e internacionais referentes ao projeto e fabricação de GGDs, de forma a garantir que o ajuste das malhas de controle aqui realizado se aproxime dos ajustes realizados pelos fabricantes dos mesmos. Uma resposta transitória típica de um regulador de velocidade se mostra na Figura 4.1 [52].

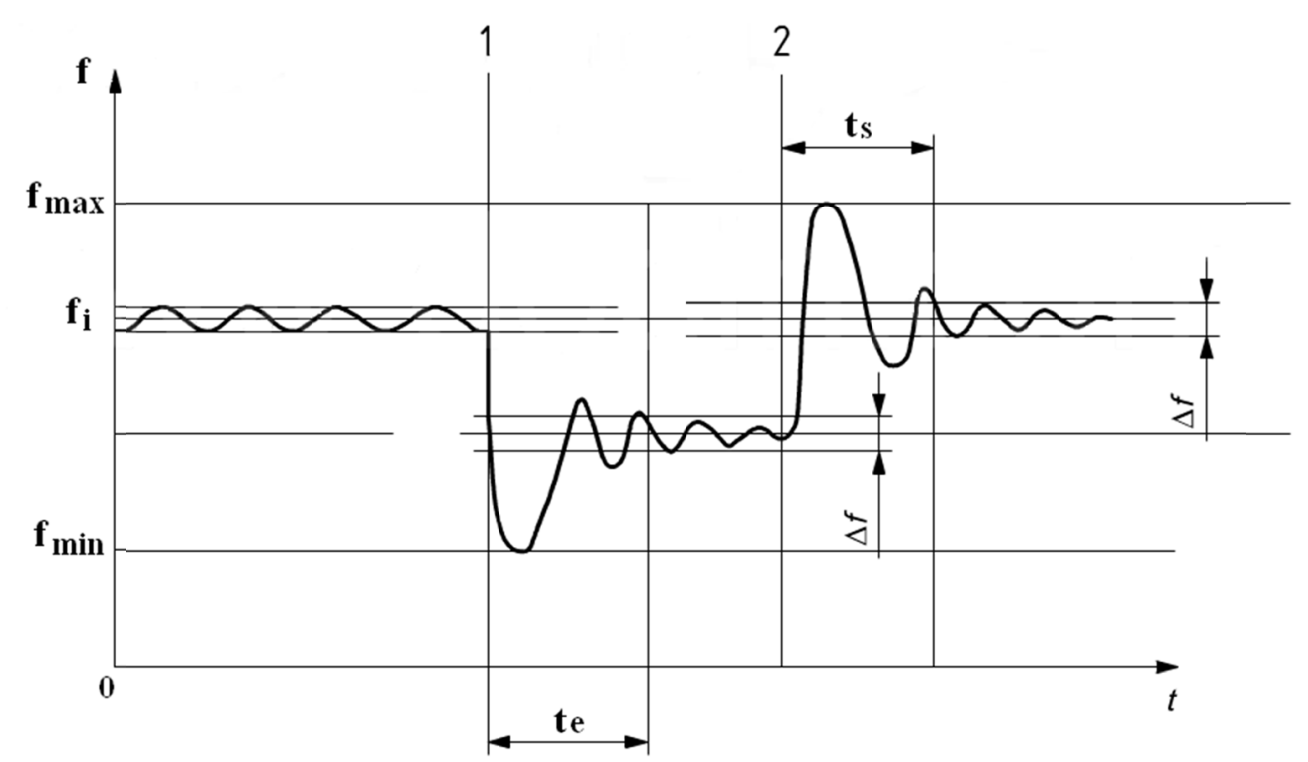

Figura 4.1 - Resposta transitória típica do regulador de velocidade - GGD

Na figura acima, $f_{\max }$ é o máximo valor transitório de frequência que o sistema pode atingir quando acontece uma perda repentina de um bloco de carga (rejeição de carga), $f_{\min }$ é o mínimo valor transitório de frequência que o sistema pode alcançar na entrada súbita de um bloco de carga (aceitação de carga), $f_{i}$ é a frequência em regime permanente do sistema para uma determinada condição de carga, $\Delta f$ é a faixa de tolerância em torno do valor de regime permanente da frequência, $t_{e}$ é 
tempo transcorrido desde o inicio de uma queda de frequência, após uma aceitação de carga, até o momento em que a frequência volta à faixa de tolerância $\Delta f \mathrm{e}$ permanece na mesma, e $t_{s}$ é o intervalo de tempo equivalente a $t_{e}$, porém, devido a uma rejeição de carga. Características de desempenho similares se aplicam ao regulador de tensão. A Tabela 4.1 mostra os valores limites para as especificações no domínio do tempo das respostas transitórias dos reguladores de tensão e velocidade segundo a norma ISO 8528-5:2005, para grupos geradores diesel classe $\mathrm{G} 3^{12}[52]$.

Tabela 4.1 - Valores limites da resposta transitória - GGDs

\begin{tabular}{ccc}
\hline Parâmetro & Frequência & Tensão \\
\hline Valor $_{\text {max }}[\%]$ & $\leq+10$ & $\leq+20$ \\
Valor $_{\min }[\%]$ & $\leq-7.0$ & $\leq-15$ \\
$\Delta[\%]$ & $\leq 0.5$ & $\leq 1.0$ \\
$\boldsymbol{t}_{\boldsymbol{e}}[\boldsymbol{s}]$ & $\leq 3.0$ & $\leq 4.0$ \\
$\boldsymbol{t}_{\boldsymbol{s}}[\boldsymbol{s}]$ & $\leq 3.0$ & $\leq 4.0$ \\
\hline
\end{tabular}

Evidentemente, os valores máximos e mínimos alcançados durante o transitório de tensão e frequência dependem da severidade da perturbação. Para isto, a norma ISO 8528-5:2005 estabelece que o GGD deve aceitar uma rejeição de 100\% da carga nominal sem ultrapassar os valores máximos mostrados na Tabela 4.1. Já a porcentagem de aceitação de carga vai depender da Pressão Efetiva Média ao Freio (BMEP) que o motor diesel acoplado ao gerador possui. O BMEP é um indicador da pressão média efetiva no interior do cilindro e um índice muito expressivo no julgamento da eficácia com que um motor tira proveito do seu tamanho (cilindrada), sendo por isso, muito usado para fins de comparação entre motores. Nem o torque nem a potência são uteis para comparar motores, pois dependem das dimensões do motor assim como da velocidade de rotação [53]. A Figura 4.2 mostra a máxima porcentagem de aceitação de carga em função do indicador BMEP para motores diesel quatro tempos [52].

12 A classe G3, segundo a norma ISO 8528-5:2005, corresponde a grupos geradores diesel projetados para atender cargas sensíveis à tensão e à frequência. 


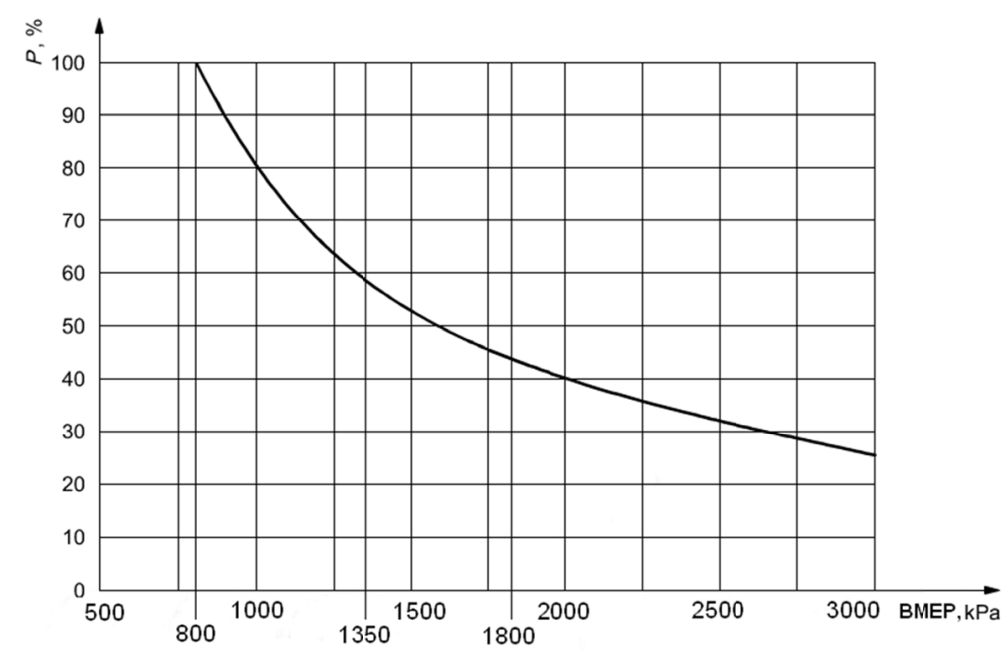

Figura 4.2 -Máxima aceitação de carga em função dlo BMEP

Neste trabalho analisaremos dois GGDs trifásicos projetados para operação continua e apropriados para conexão em redes de BT de 220/127 V - $60 \mathrm{~Hz}$. Os parâmetros elétricos e mecânicos dos GGDs se mostram na Tabela 4.2 [39] [51].

Tabela 4.2 - Parâmetros elétricos e mecânicos dos: GGDs

\begin{tabular}{ccc}
\hline Parâmetro & GGD1 & GGD2 \\
\hline $\boldsymbol{S}[\boldsymbol{k V A} \boldsymbol{A}] / \boldsymbol{f p}$ & $45 / 0.8$ & $90 / 0.8$ \\
$\boldsymbol{H}_{\boldsymbol{s}}[\boldsymbol{s}] / \boldsymbol{K}_{\boldsymbol{S} \boldsymbol{D}}[\boldsymbol{p u}] / \boldsymbol{p o l o s}$ & $0.5 / 0.02 / 4$ & $0.48 / 0.02 / 4$ \\
$\boldsymbol{B M E P}[\boldsymbol{k P a}]$ & 1020 & 13.35 \\
$\boldsymbol{L}_{\boldsymbol{l}}[\boldsymbol{p u}]$ & 0.07 \\
$\boldsymbol{L}_{\boldsymbol{s} \boldsymbol{d}} / \boldsymbol{L}_{\boldsymbol{s} \boldsymbol{d}^{\prime}} / \boldsymbol{L}_{\boldsymbol{s} \boldsymbol{d}}{ }^{\prime \prime}[\boldsymbol{p u}]$ & $2.67 / 0.21 / 0.12$ \\
$\boldsymbol{L}_{\boldsymbol{s q}} / \boldsymbol{L}_{\boldsymbol{s q}}^{\prime \prime}[\boldsymbol{p u}]$ & $1.60 / 0.15$ \\
$\boldsymbol{t}_{\boldsymbol{a}} / \boldsymbol{t}_{\boldsymbol{d}}^{\prime} / \boldsymbol{t}_{\boldsymbol{d}}^{\prime \prime} / \boldsymbol{t}_{\boldsymbol{q}}^{\prime \prime}[\boldsymbol{m s}]$ & $15 / 100 / 10 / 10$ \\
$\boldsymbol{T}_{\boldsymbol{A}} / \boldsymbol{T}_{\boldsymbol{D}}[\boldsymbol{m s}]$ & $40 / 100$ \\
$\boldsymbol{D r o o p}[\%]$ & 4.0 \\
\hline
\end{tabular}

Na tabela acima, $L_{s d}^{\prime}$ e $L_{s d}^{\prime \prime}$ representa a indutância transitória e subtransitória de eixo direto, $L_{s q}$ " é a indutância subtransitória de eixo de quadratura, $t_{a}$ representa a constante de tempo de armadura, $t_{d}^{\prime}$ e $t_{d}{ }^{\prime \prime}$ a constante de tempo transitória e subtransitória de curto-circuito de eixo direto e $t_{q}{ }^{\prime \prime}$ a constante de tempo subtransitória de curto-circuito de eixo de quadratura. Os outros símbolos foram definidos no Capítulo 3.1. Estes parâmetros (conhecidos como parâmetros 
operacionais do gerador síncrono) são determinados a partir de ensaios realizados sobre a máquina e fornecem as características da resposta dinâmica do gerador. Todavia, no modelo apresentado no Capitulo 3.1, as equações da máquina foram desenvolvidas em termos das indutâncias e resistências dos circuitos do rotor e do estator (parâmetros fundamentais da máquina), consequentemente faz se necessário realizar a respectiva conversão. É importante mencionar que na literatura especializada [29] [30] [34] é comum encontrar simplificações no processo de resolução do sistema de equações que relaciona os parâmetros fundamentais com os operacionais, permitindo obter com bastante precisão os parâmetros fundamentais da máquina com pouco esforço computacional. Estas simplificações se baseiam nas relações usualmente existentes (em pu) entre a resistência de campo e as resistências dos enrolamentos amortecedores em máquinas de grande porte $\left(R_{1 d} \gg R_{1 q} \gg R_{f d}\right)$. Entretanto, essas simplificações podem acarretar erros significativos no caso de geradores de pequeno porte. Assim sendo, viu se a necessidade de resolver o sistema de equações que relacionam os parâmetros operacionais com os fundamentais sem nenhum tipo de simplificação através de métodos computacionais iterativos. Os resultados se mostram na Tabela 4.3.

Tabela 4.3 - Parâmetros elétricos fundamentais dos GGDs

\begin{tabular}{cc}
\hline Parâmetro & G1 e G2 \\
\hline $\boldsymbol{R}_{\boldsymbol{a}} / \boldsymbol{L}_{\boldsymbol{l}}[\boldsymbol{p u}]$ & $0.02387 / 0.07$ \\
$\boldsymbol{L}_{\boldsymbol{a d}} / \boldsymbol{L}_{\boldsymbol{a q}}[\boldsymbol{p u}]$ & $2.6 / 1.53$ \\
$\boldsymbol{R}_{\boldsymbol{f d}} / \boldsymbol{L}_{\boldsymbol{f d}}[\boldsymbol{p u}]$ & $0.00697 / 0.19$ \\
$\boldsymbol{R}_{\mathbf{1 d}} / \boldsymbol{L}_{\mathbf{1 d}}[\boldsymbol{p u}]$ & $0.03125 / 0.06968$ \\
$\boldsymbol{R}_{\mathbf{1 q}} / \boldsymbol{L}_{\mathbf{1 q}}[\boldsymbol{p u}]$ & $0.04015 / 0.08441$ \\
\hline
\end{tabular}

De posse destas informações, procede-se ao ajuste das malhas de controle dos reguladores de velocidade e tensão. Embora seja possível realizar um ajuste empírico dos parâmetros de controle, este procedimento demandaria muito tempo e ainda poderia levar a valores que não seriam necessariamente os melhores. Em vez disso, optou-se por definir uma função custo (FC) capaz de avaliar quantitativamente a resposta dinâmica do sistema, tanto para o cenário de rejeição de carga quanto para o de aceitação. As FCs são compostas pela união das características da resposta transitória mostradas na Tabela 4.1 da seguinte forma: 


$$
\begin{gathered}
F C_{\text {rejeição }}=\alpha \cdot\left(f_{\max }+v_{\max }\right)+\beta \cdot(\Delta f+\Delta v)+\gamma \cdot\left(t_{s f r e q}+t_{\text {stensão }}\right) \\
F C_{\text {aceitação }}=\alpha \cdot\left(f_{\min }+v_{\text {min }}\right)+\beta \cdot(\Delta f+\Delta v)+\gamma \cdot\left(t_{\text {freq }}+t_{\text {etensão }}\right)
\end{gathered}
$$

Onde as constantes $\alpha, \beta$ e $\gamma$ são os pesos atribuídos a cada termo das FCs. Uma vez obtida uma função custo bem definida, técnicas de otimização podem ser aplicadas visando obtenção dos parâmetros das malhas de controle dos reguladores de tensão e frequência que determinem uma estabilidade suave e rápida do sistema. Neste trabalho, utilizou-se os algoritmos disponíveis no Toolbox de Otimização do software Matlab ${ }^{\circledR}$. A simulação no domínio do tempo foi computada através do software Simulink $\circledast$, utilizando-se os modelos não lineares próprios da biblioteca SimPowerSystems. Os sistemas a otimizar, formados pelos GGDs ligados diretamente a uma carga de impedância constante R-L com fator de potência (fp) 0.8, são testados aplicando-se um degrau de rejeição de carga de $100 \%$, e uma aceitação de carga de 75\% para o GGD1 e 60 \% para o GGD2; valores calculados em função do indicador BMEP. Para este teste, o controle secundário de frequência é desligado ${ }^{13}$ (i.e., $K_{i m-S}=0$ ). A Tabela 4.4 mostra os indicadores de desempenho dos sistemas otimizados.

Tabela 4.4 - Desempenho do sistema GGD+Carga R-L

\begin{tabular}{ccccc}
\hline \multirow{2}{*}{ Parâmetro } & \multicolumn{2}{c}{ GGD1 } & \multicolumn{2}{c}{ GGD2 } \\
& Freq. & Tensão & Freq. & Tensão \\
\hline Valor $_{\max }[\%]$ & +4.2 & +19.3 & +4.5 & +19.0 \\
Valor $_{\min }[\%]$ & -5.0 & -12.8 & -4.73 & -10.6 \\
$\boldsymbol{\Delta}[\%]$ & 0.5 & 1.0 & 0.5 & 1.0 \\
$\boldsymbol{t}_{\boldsymbol{e}}[\boldsymbol{s}]$ & 1.11 & 1.30 & 1.09 & 1.29 \\
$\boldsymbol{t}_{\boldsymbol{s}}[\boldsymbol{s}]$ & 2.71 & 2.68 & 2.92 & 3.09 \\
\hline
\end{tabular}

\footnotetext{
${ }^{13}$ A Norma ISO 8528-5:2005 não faz menção explicita ao controle secundário dos GGDs, porém, da Figura 4.1 é possível inferir que não existe um controle secundário responsável por restabelecer a frequência ao valor programado nos testes de rejeição e aceitação de carga.
} 
A análise da Tabela 4.4 mostra que os ajustes das malhas de controle são satisfatórios, atendendo as especificações da Norma ISO 8528:-5:2005. As Figuras 4.3 à 4.6 mostram as respostas transitórias de velocidade e tensão para o GGD1. Respostas similares foram obtidas para o GGD2

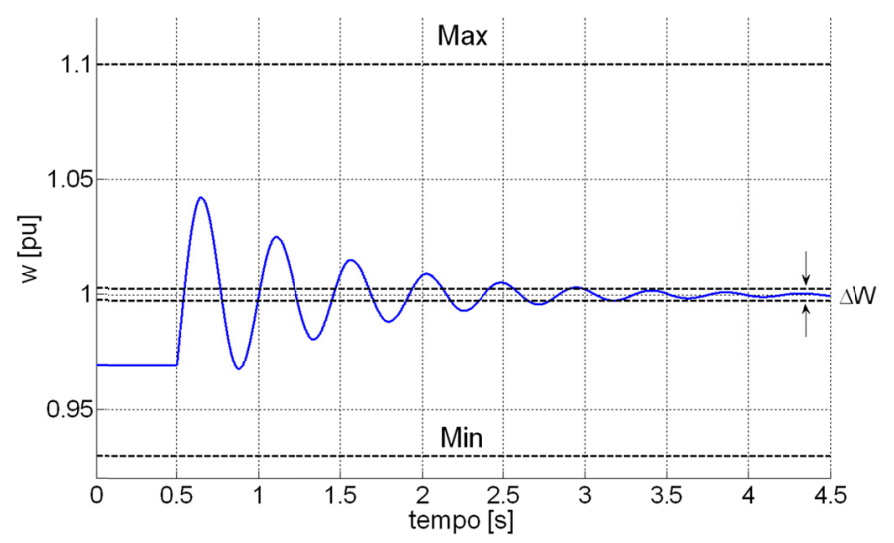

Figura 4.3 - Resposta transitória de velocidade GGD1- rejeição carga R-L

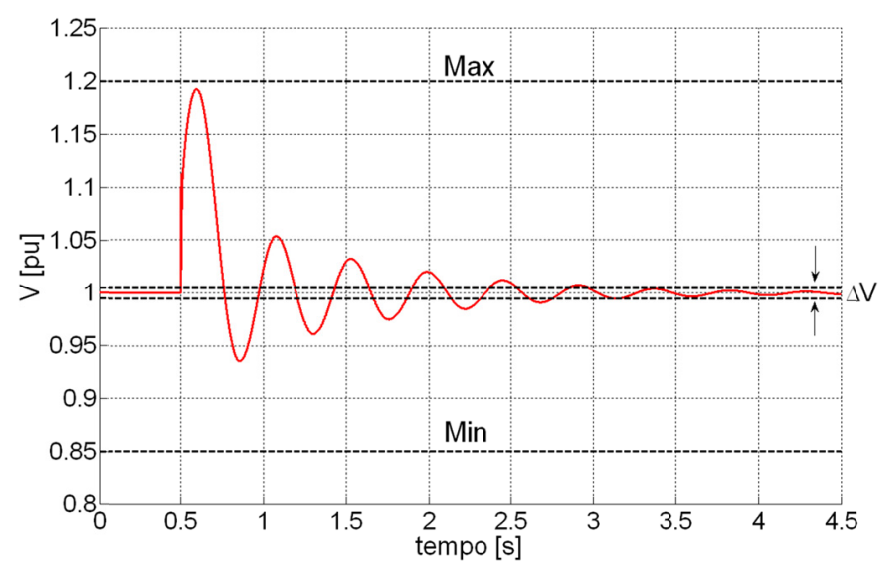

Figura 4.4 - Resposta transitória de tensão GGD1 - rejeição carga R-L

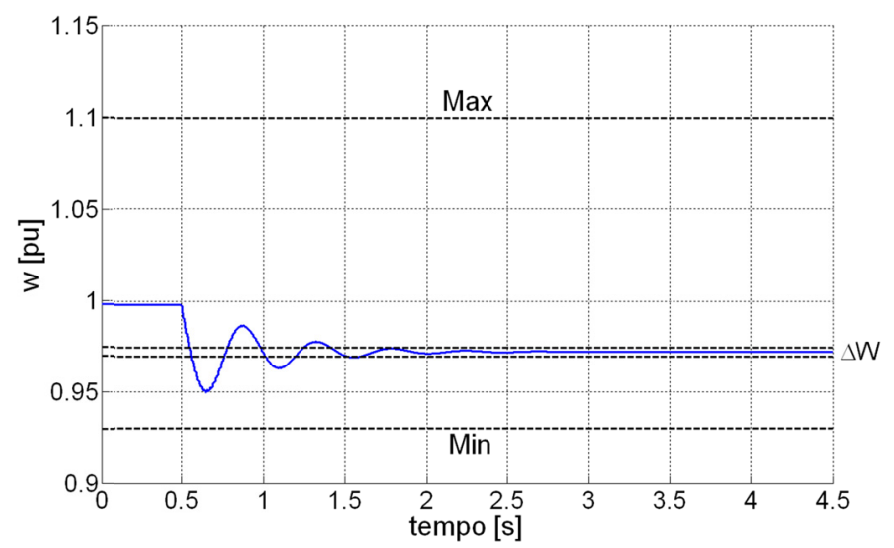

Figura 4.5 - Resposta transitória de velocidade GGD1 - aceitação carga 


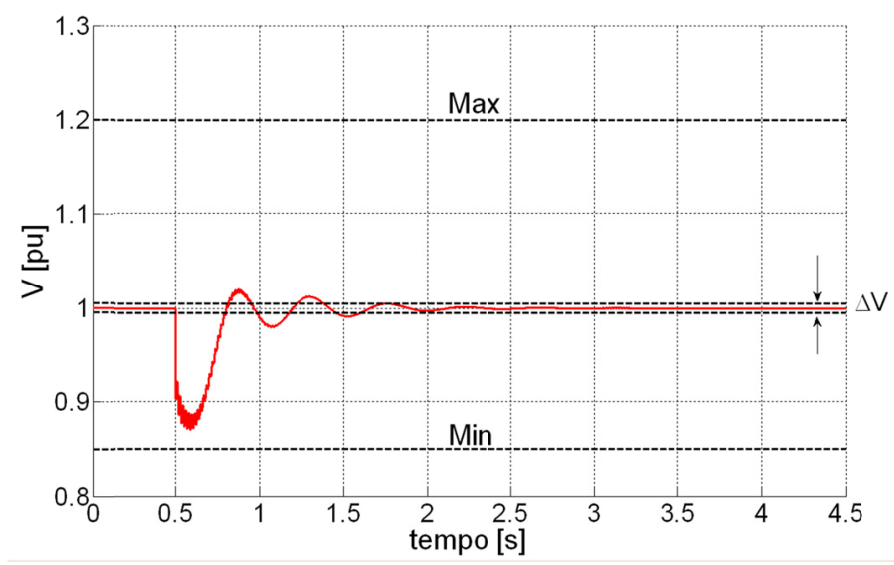

Figura 4.6 - Resposta transitória de tensão GGD1 - aceitação de carga

Um projeto adequado do controle secundário de frequência deve garantir sua atuação unicamente depois de passado o transitório eletromecânico, onde temos basicamente a atuação do controle primário. Para o caso dos GGDs considera-se apropriado o restabelecimento da frequência ao seu valor programado na faixa de 10 segundos após um distúrbio [7]. Assim sendo, o ajuste da malha de controle secundário é realizado manualmente através de simulações no domínio do tempo, ajustando o valor do parâmetro $K_{i m-S}$ de forma a restabelecer a frequência a $60 \mathrm{~Hz}$ em 10 segundos após uma aceitação de carga de $75 \%$ para o GGD1 e $60 \%$ para o GGD2.

Os ganhos finais das malhas de controle dos reguladores de velocidade e tensão se mostram na Tabela 4.5.

Tabela 4.5 - Ganhos das malhas de controle - GGD

\begin{tabular}{ccc}
\hline Parâmetro & GGD1 & GGD2 \\
\hline $\boldsymbol{K}_{p v-S} / \boldsymbol{K}_{i v-S}$ & $0.7244 / 79.366$ & $0.615 / 83.68$ \\
$\boldsymbol{K}_{\boldsymbol{F} F} / \boldsymbol{K}_{\boldsymbol{M}}$ & $1.4846 / 1.324$ & $1.4035 / 1.546$ \\
$\boldsymbol{K}_{\boldsymbol{G}} / \boldsymbol{T}_{\boldsymbol{G}}$ & $1.4972 / 0.1765$ & $1.2741 / 0.1434$ \\
$\boldsymbol{R}_{\boldsymbol{p - s}} / \boldsymbol{K}_{\boldsymbol{i m - S}}$ & $0.04 / 10$ & $0.04 / 10$ \\
\hline
\end{tabular}




\subsubsection{Análise da estabilidade dinâmica}

\subsubsection{Carga Passiva - R-L}

Ajustadas as malhas de controle procede-se a analisar a estabilidade dinâmica dos GGDs através do enfoque dos autovalores da matriz de estado. A Tabela 4.6 mostra os autovalores correspondentes à operação pré-distúrbio nominal dos GGDs, com carga $R-L, f p=0.8$, diretamente ligada aos terminais do gerador. $O$ sistema é descrito por doze variáveis de estado associadas aos modelos no espaço de estados do GGDs e duas variáveis de estado associadas aos modelos no espaço de estados das cargas, consequentemente, tem-se catorze autovalores $(\lambda)$ para cada caso. Como pode se observar, com exceção do $\lambda_{14}$, todos os autovalores tem parte real negativa, indicando uma condição de estabilidade para o ponto de operação considerado. O autovalor igual a zero $\left(\lambda_{14}\right)$ está associado com a falta de uma referência absoluta para a medição do ângulo do rotor (o sistema não contem uma barra infinita) e não fornece nenhuma informação sobre as características dinâmicas do sistema.

Tabela 4.6 - Autovalores do Sistema - GGD + Carga nominal R-L

\begin{tabular}{ccc}
\hline Autovalor & GGD1 & GGD2 \\
\hline 1 & $-1.40 \mathrm{e} 6$ & $-3.30 \mathrm{e} 6$ \\
2 & $-1.40 \mathrm{e} 6$ & $-2.76 \mathrm{e} 6$ \\
3,4 & $-435.27 \pm \mathrm{j} 370.25$ & $-435.27 \pm \mathrm{j} 370.34$ \\
5 & -59.579 & -59.907 \\
6 & -33.072 & -33.260 \\
7 & -20.393 & -21.627 \\
8,9 & $-1.535 \pm \mathrm{j} 13.077$ & $-1.385 \pm \mathrm{j} 13.367$ \\
10,11 & $-3.386 \pm \mathrm{j} 10.100$ & $-3.276 \pm \mathrm{j} 9.808$ \\
12 & -7.207 & -9.763 \\
13 & -0.4298 & -0.4286 \\
14 & 0 & 0 \\
\hline
\end{tabular}


A Figura 4.7 mostra os principais autovalores dos sistemas plotados no plano complexo, onde as linhas inclinadas pontilhadas representam o lugar geométrico dos pontos com fator de amortecimento $(\zeta)$ constante e as semi-elipses pontilhadas indicam o lugar geométrico dos pontos com frequência natural $\left(w_{n}\right)$ de oscilação constante.
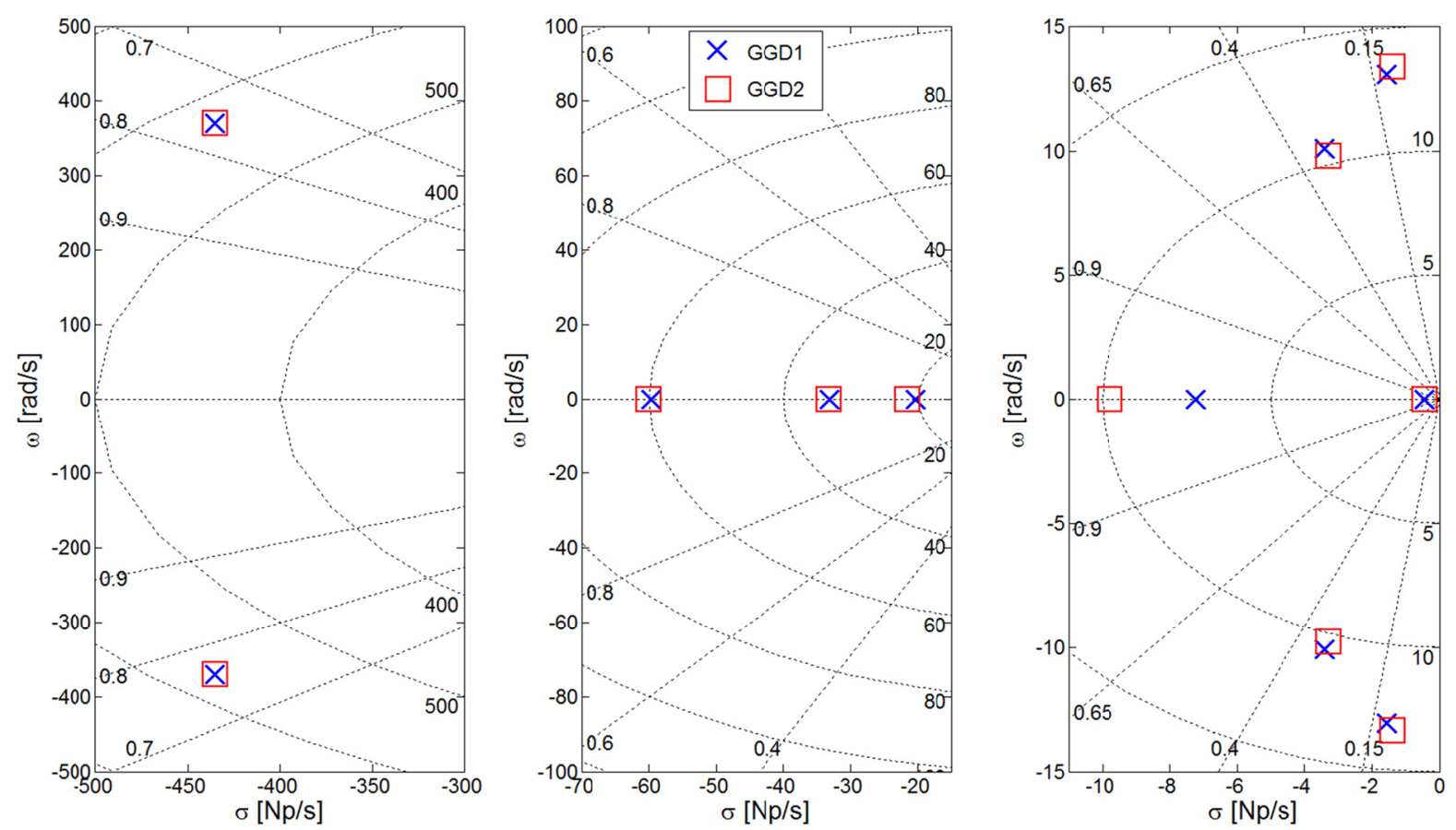

Figura 4.7 - Autovalores do Sistema - GGD + Carga nominal R-L

De forma geral observa-se que a disposição dos autovalores no plano complexo é muito similar para os dois GGDs em estudo, observando-se a maior diferença no $\lambda_{12}$. Por outra parte, a análise da Figura 4.7 mostra a existência de dois pares de autovalores $\left(\lambda_{8,9}\right.$ e $\left.\lambda_{10,11}\right)$ com baixo $\zeta(<0.4)$, que dominam a resposta transitória do sistema. O primeiro par apresenta um $\zeta$ em torno de $0.11 \mathrm{com} w_{n}$ na faixa de 2.13 $\mathrm{Hz}$ enquanto o segundo par exibe um $\zeta$ em torno de $0.3 \mathrm{com} w_{n}$ na faixa de $1.65 \mathrm{~Hz}$. Os modos oscilatórios associados a estes autovalores são conhecidos como modos eletromecânicos de baixa frequência e, quando mal amortecidos, são prejudiciais para a operação dos sistemas elétricos de potência, podendo causar a perda de sincronismo dos geradores. Geralmente, o $\zeta$ destes modos limita-se a um valor mínimo de 0.05 para operação aceitável dos sistemas de potência. Já os 
autovalores restantes apresentam um elevado $\zeta$, não influenciando significativamente a resposta transitória do sistema.

Para uma melhor análise foram calculados os fatores de participação das variáveis de estado nos modos do sistema. O resultado se mostra na Figura 4.8, onde os fatores de participação com valor inferior a 0.1 foram suprimidos por conveniência. Um gráfico similar pode ser obtido para o GGD2.

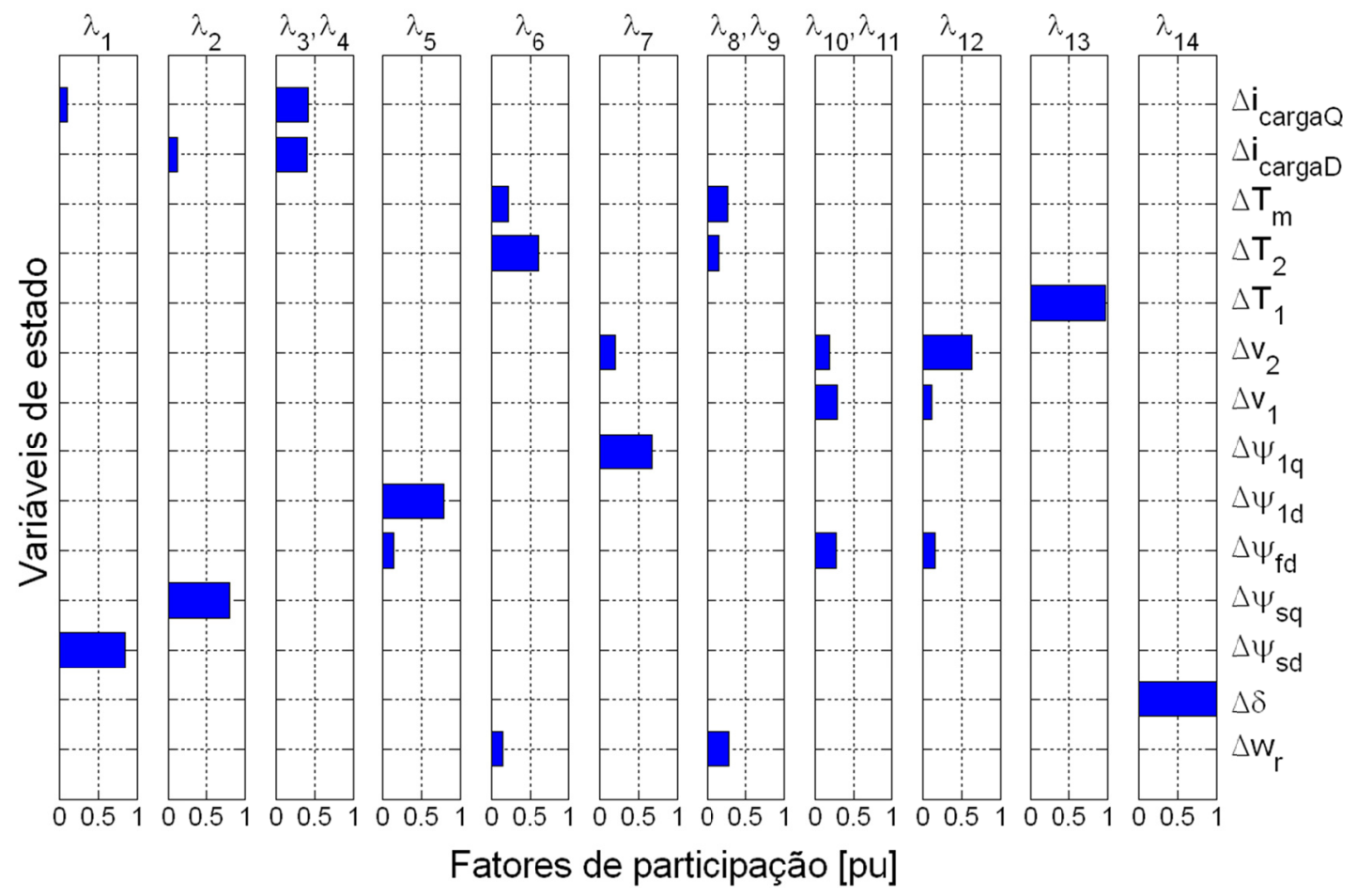

Figura 4.8 - Fatores de Participação - GGD1+Carga nominal R-L

Observa-se que os autovalores reais $\lambda_{1}$ e $\lambda_{2}$ estão associados diretamente às variações nos enlaces de fluxo do estator, são autovalores não oscilatórios com constantes de tempo muito pequenas. O par de autovalores complexos $\lambda_{3,4}$ está relacionado às variações na corrente da carga (e, portanto, também às correntes do estator) e têm frequência amortecida próxima a $60 \mathrm{~Hz}$. Estes modos estão associados com as correntes transitórias em CC presentes nos enrolamentos do estator e na carga imediatamente após um distúrbio. Estas correntes de CC são refletidas como componentes de frequência fundamental na referência rotativa d-q, gerando um torque pulsatório responsável pelas oscilações em $60 \mathrm{~Hz}$. Os autovalores reais $\lambda_{5}$ e $\lambda_{7}$ estão associados com as correntes transitórias induzidas 
nos enrolamentos amortecedores de eixo direto e de quadratura respectivamente. $\mathrm{O}$ $\lambda_{5}$ apresenta uma constante de tempo na ordem de $16 \mathrm{~ms}$ enquanto $\circ \lambda_{7}$ de $47 \mathrm{~ms}$.

Um dos modos eletromecânicos $\left(\lambda_{10,11}\right)$ está relacionado aos enlaces de fluxo do enrolamento de campo assim como a malha externa de controle do sistema de excitação. Isto mostra que sob condições oscilatórias, as variações na tensão de excitação do campo causadas pela ação do regulador de tensão, têm uma influência sobre o amortecimento da máquina. Lamentavelmente, como explicado a seguir, estas variações afetam negativamente a estabilidade dinâmica do sistema.

Quando o ângulo do rotor $\left(\delta_{r}\right)$, também conhecido como ângulo de carga, aumenta, a corrente do estator se incrementa, incrementando também a queda de tensão na impedância do gerador. Esta redução é detectada pelo regulador de tensão, que faz com que o sistema de excitação aumente a tensão de campo, produzindo um incremento nos enlaces de fluxo do eixo $d$ para compensar a queda de tensão. Infelizmente os enlaces de fluxo não podem ser incrementados instantaneamente, consequentemente, durante condições oscilatórias, as variações nos enlaces de fluxo atrasam as variações na tensão de campo e, portanto, as variações no $\delta_{r}$. Assim sendo, devido à ação do regulador de tensão, uma componente de torque contrária ao desvio de velocidade do rotor é produzida, minimizando o amortecimento natural do gerador. Para este caso, a constante de tempo do modo $\lambda_{10,11}$ está na faixa de $300 \mathrm{~ms}$.

Por outra parte, é importante observar que o autovalor real $\lambda_{12}$ está associado à malha interna estabilizadora do controle de excitação, com constante de tempo na ordem de $125 \mathrm{~ms}$, i.e., aproximadamente 2.5 vezes inferior ao da malha externa. Como resultado, a constante de tempo efetiva associado ao sistema de excitação é reduzida, minimizando assim o atraso existente entre às variações na tensão do campo e os enlaces de fluxo, melhorando o desempenho dinâmico do sistema.

O segundo modo eletromecânico $\left(\lambda_{8,9}\right)$ está associado à dinâmica do motor diesel e do regulador de velocidade (especificamente ao controle primário de frequência). Sob condições oscilatórias, às variações na velocidade do rotor detectadas pelo regulador de velocidade, se traduzem em variações no torque mecânico de saída do motor diesel. Teoricamente, se o sistema motor-regulador de velocidade tivesse uma 
resposta instantânea, as variações no torque mecânico estariam completamente em fase com as variações de velocidade e consequentemente teriam uma contribuição positiva direta no amortecimento do GGD. Na prática, a resposta dinâmica associada ao sistema motor-regulador de velocidade é relativamente lenta, e o atraso produz uma defasagem entre as variações de velocidade e as variações do torque mecânico, diminuindo a componente do torque amortecedor. Para ângulos de defasagem maiores a $90^{\circ}$, as variações no torque mecânico geram uma componente de torque amortecedor contrária às variações de velocidade, com contribuição negativa direta no amortecimento do GGD. Para o caso em estudo, a constante de tempo deste modo está na faixa de $700 \mathrm{~ms}$.

$O$ autovalor real $\lambda_{6}$ está mais diretamente relacionado ao atuador do regulador de velocidade, com constante de tempo muito menor, na faixa de $30 \mathrm{~ms}$. Já o autovalor real $\lambda_{13}$ está diretamente associado ao controle secundário de frequência, e como esperado, apresenta a maior constante de tempo do sistema ( 2300 ms). Finalmente, como já explicado, pode se observar que o autovalor $\lambda_{14}$ está unicamente associado à posição angular do rotor com relação à referência.

Após analisar a estabilidade dinâmica dos GGDs para condições pré-distúrbio nominais, é de interesse também estudar o desempenho dinâmico do sistema para outras condições operativas. A Figura 4.9 e Figura 4.10 mostram o lugar geométrico da parte real $(\sigma)$, imaginária $(w)$, fator de amortecimento $(\zeta)$, e frequência natural $\left(w_{n}\right)$ dos modos eletromecânicos, para variações na potência ativa e no fator de potência da condição pré-distúrbio do GGD.

A análise das figuras mostra que o sistema é estável $(\sigma<0)$ para todas as condições pré-distúrbio analisadas, entretanto observam-se variações no $\zeta$ dos autovalores. No caso do $\lambda_{10,11}$, nota-se uma diminuição do $\zeta$ com o aumento de P. Esse comportamento está associado ao incremento do ângulo do rotor $\left(\delta_{r}\right)$. Para pequenos valores de $\delta_{r}$, o fluxo resultante no entreferro está praticamente alinhado com o eixo $d$, sendo a força eletromotriz (fem) induzida no enrolamento amortecedor do eixo $q$ máxima durante as oscilações do rotor. À medida que o $\delta_{r}$ aumenta (devido ao aumento de P), a componente do eixo $d$ do fluxo resultante torna-se 
menor, reduzindo a fem induzida no enrolamento amortecedor do eixo $q$ e aumentando a fem induzida no enrolamento amortecedor do eixo $d$.
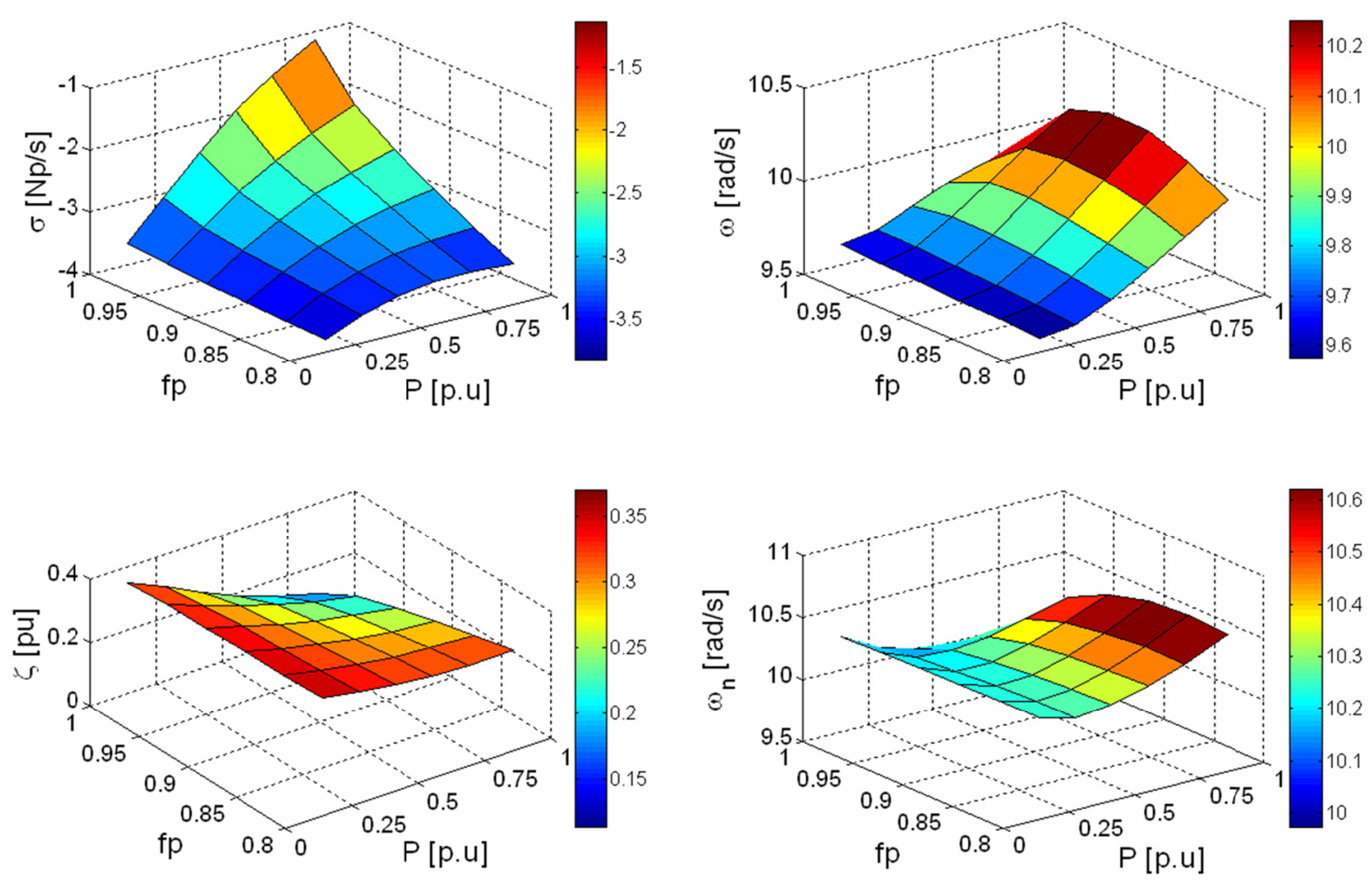

Figura 4.9 - Lugar geométrico do $\lambda_{10,11}$ para variações de $P$ e fp - GGD1

Pelo fato da resistência do amortecedor do eixo $q$ ser muito menor que a do eixo $d$, para um mesmo valor de fem a corrente induzida no enrolamento do eixo $q$ é maior daquela induzida no eixo $d$, consequentemente o torque amortecedor é maior. Disto se deduz que o incremento da fem no eixo d não compensa totalmente a perda de fem no eixo q, reduzindo-se assim o amortecimento líquido da máquina com o aumento de $P$.

O aumento do $\delta_{r}$ também está associado ao fator de potência no qual opera a máquina síncrona. À medida que o fp da carga aumenta, o $\delta_{r}$ também aumenta, reduzindo levemente o amortecimento líquido do gerador como mostra a Figura 4.9. O menor amortecimento para o modo eletromecânico $\lambda_{10,11}$ se tem no ponto de operação $P=1.0$ pu e fp=1.0, $\operatorname{com} \zeta=0.11$. Já o $w_{n}$ apresenta pouca variação em função do ponto de operação da máquina. 

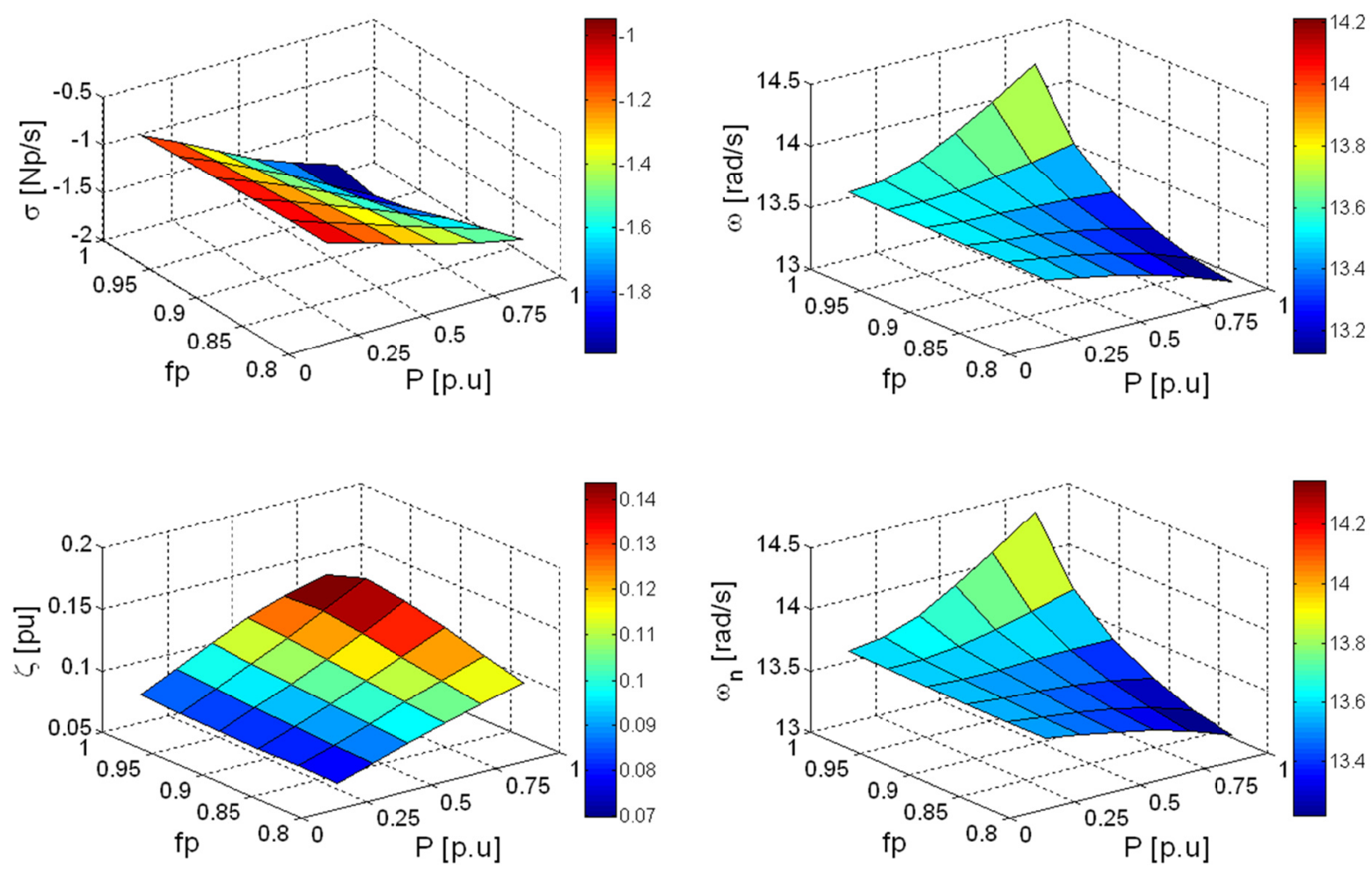

Figura 4.10 - Lugar geométrico do $\lambda_{\mathbf{8}, 9}$ para variações de P e fp - GGD1

Por outra parte, a análise da Figura 4.10 mostra um comportamento contrário para o $\zeta$ do modo $\lambda_{8,9}$ em função da variação de $P$, se comparado com o $\lambda_{10,11}$. Neste caso verifica-se uma perda significativa do $\zeta$ em função do decremento de $\mathrm{P}$, chegandose a valores na ordem de 0.07 para operação em vazio da máquina. Entretanto, este modo mostra-se praticamente insensível as variações do fp da carga.

Para corroborar os dados obtidos através da análise modal, simulações na plataforma Simulink® foram realizadas com modelos não lineares detalhados do sistema. Os resultados das simulações no domínio do tempo foram então comparados com os correspondentes resultados obtidos do modelo linear. A Figura 4.11 mostra as respostas no domínio do tempo de algumas das grandezas do sistema, para uma aceitação de carga de 15\% para condição pré-distúrbio nominal. Embora este valor possa parecer elevado para uma análise de estabilidade angular a pequenos distúrbios, em sistemas de potência de pequeno porte (como é o caso das minirredes) o simples fato de ligar ou desligar algum equipamento elétrico pode facilmente produzir variações na carga desta magnitude, consequentemente é importante validar a aplicabilidade da análise modal sob essas circunstâncias. 

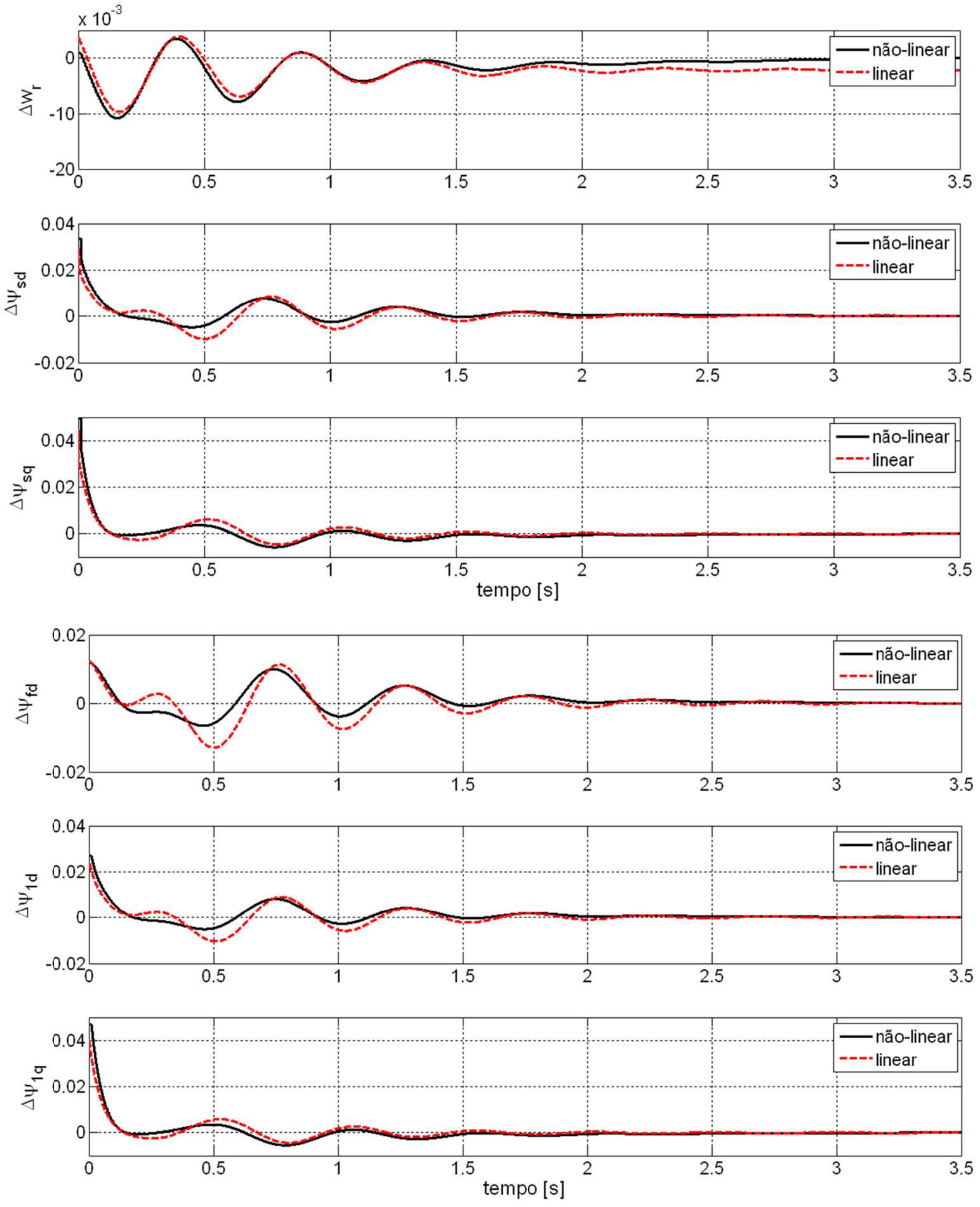

Figura 4.11 - Resposta transitória para 15\% de variação na carga - GGD1

A comparação dos resultados obtidos através do modelo não linear com os resultados obtidos do modelo linear indica um padrão semelhante de comportamento das variáveis do sistema, com máximo sobrepasso (overshoot), tempo de assentamento (settling time) e constantes de tempo praticamente similares 
para os dois casos, até mesmo para valores elevados de distúrbio (15\%). Desta forma, verifica-se qualitativamente a validade do modelo linear nas análises anteriormente realizadas.

É importante observar que nos ajustes realizados as malhas de controle, os parâmetros de controle do regulador de velocidade $\left(R_{p-S}\right.$ e $\left.K_{i m-S}\right)$ não foram otimizados em função da resposta transitória dos GGDs. O valor de $R_{p-S}$ (droop de velocidade) foi obtido diretamente do catálogo do fabricante ${ }^{14}$. Já o valor de $K_{i m-S}$ foi ajustado empiricamente de forma a restabelecer a frequência ao seu valor programado em aproximadamente 10 segundos após um distúrbio. Sendo assim, considera-se importante realizar uma análise de sensibilidade dos modos eletromecânicos a estes parâmetros. A Figura 4.12 mostra o lugar geométrico dos modos eletromecânicos para variações no droop de velocidade entre 3\% e 5\% (em passos de $0.4 \%$ ), para operação nominal do GGD1. As setas indicam o sentido de incremento do valor de droop ${ }^{15}$.

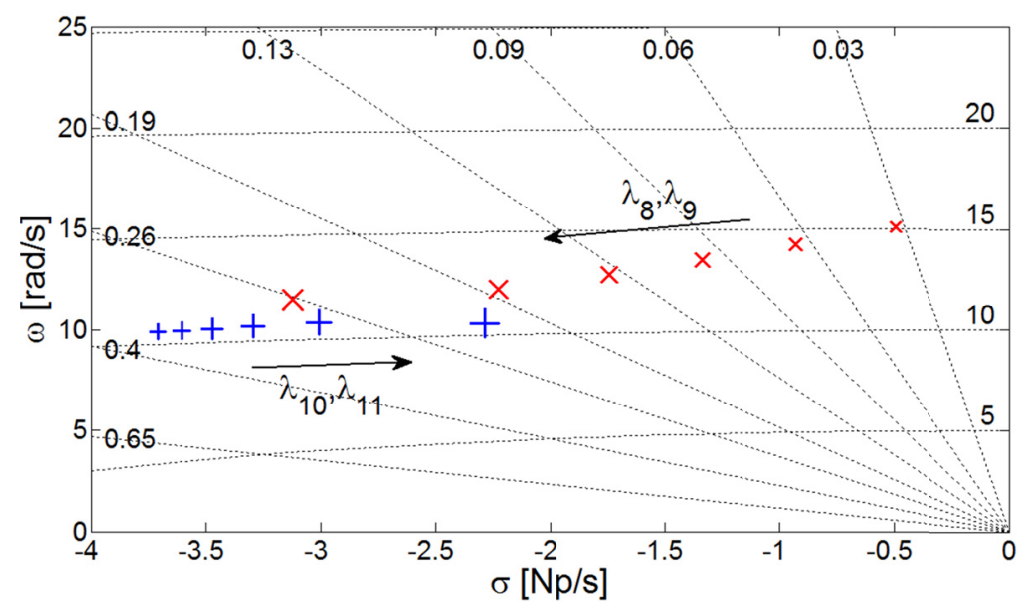

Figura 4.12 - Lugar geométrico do $\lambda_{8,9} e \lambda_{10,11}$ para variações de $\boldsymbol{R}_{p-S}-$ GGD1

Como se pode observar, as direções de migração dos modos eletromecânicos são opostas em função das variações do droop de velocidade. Para o caso em estudo, um valor de droop de $4.6 \%$ apresenta o melhor compromisso de $\zeta$ para os dois

\footnotetext{
${ }^{14}$ No Brasil (e EUA) este parâmetro é usualmente fixado em 5\%, sendo na Europa $4 \%$ o valor normalmente empregado.

${ }^{15}$ No que se segue, as setas sempre indicarão o sentido de incremento de uma variável ou ganho.
} 
modos. Valores de droop inferiores a $3 \%$ são inadmissíveis, pois deixam o modo $\lambda_{8,9}$ pouco amortecido $(\zeta<0.05)$. Por outra parte, nota-se que a frequência de oscilação do modo $\lambda_{8,9}$ é sensível às variações no droop, diminuindo $w$ com o incremento do droop. Já a frequência de oscilação do $\lambda_{10,11}$ mostra-se praticamente insensível as mudanças no valor do droop.

Para validar estes resultados, simulações no domínio do tempo na plataforma Simulink ${ }^{\circledR}$, com modelos não lineares detalhados do sistema, foram realizadas para o GGD1, considerando três diferentes valores de droop de velocidade. Para todos os casos simulados, o distúrbio aplicado ao sistema corresponde a uma aceitação de carga de $25 \%$ no tempo $t=0.5 \mathrm{~s}$, para condição pré-distúrbio do gerador: $\mathrm{P}=0.75$ pu e $\mathrm{fp}=0.8$. Os resultados se mostram na Figura 4.13.

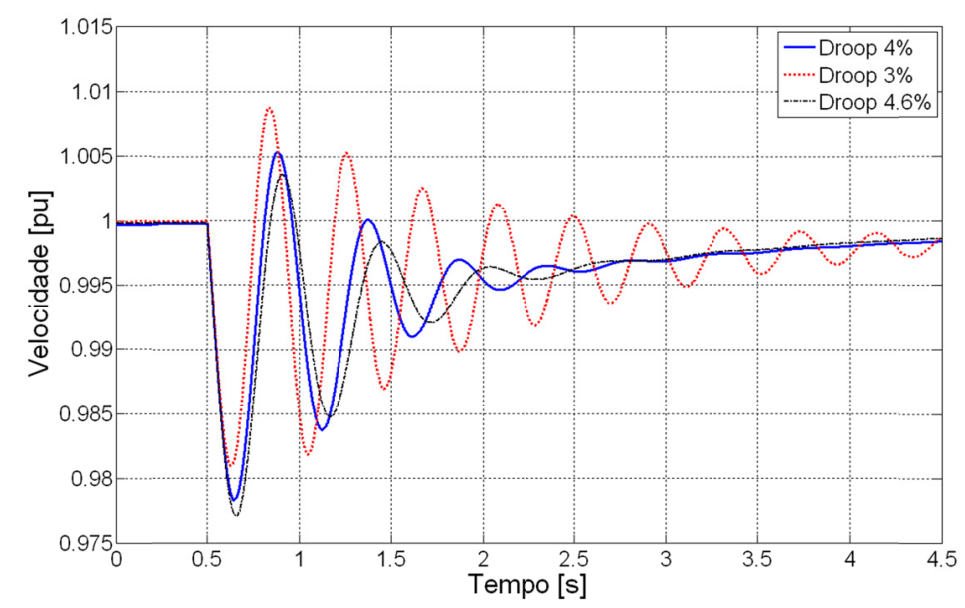

Figura 4.13 - Resposta transitória de velocidade para variações de $\boldsymbol{R}_{p-s}-$ GGD1

Como a análise modal mostrou, o valor do droop tem uma influência direta na resposta transitória do sistema, podendo se observar que valores de droop abaixo de $3 \%$ produzem oscilações sustentadas por vários segundos após o distúrbio. Já a diferença na resposta dinâmica, para os valores de droop de $4.6 \%$ e $4.0 \%$, é mínima, registrando-se uma leve melhoria no amortecimento do sistema para o primeiro valor. Entretanto, observa-se que o incremento do droop deixa as oscilações do sistema mais lentas, variando de $2.3 \mathrm{~Hz}$ (droop = 3\%) para $1.85 \mathrm{~Hz}$ $($ droop $=4.6 \%)$. 
A Figura 4.14 mostra o lugar geométrico dos modos eletromecânicos para variações no ganho do controle secundário (i.e., no valor de $K_{i m-s}$ ) entre 0 e 25 pu, em passos de 5 pu.

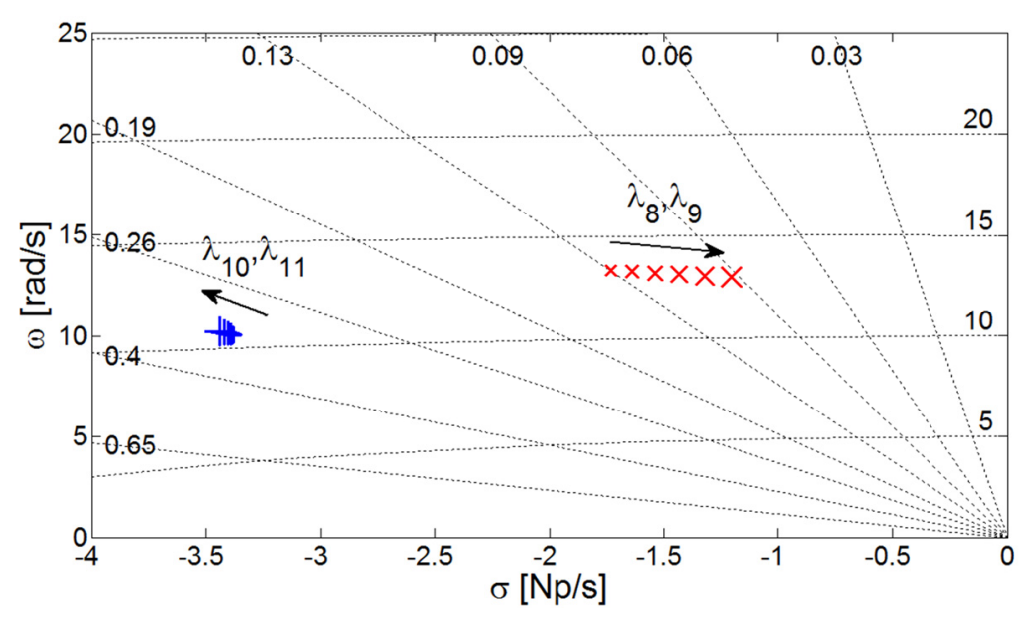

Figura 4.14 - Lugar geométrico do $\lambda_{\mathbf{8 , 9}} e \lambda_{10,11}$ para variações de $\boldsymbol{K}_{\boldsymbol{i m - S}}-\mathrm{GGD1}$

A análise da Figura 4.14 mostra que o modo $\lambda_{10,11}$ é praticamente insensível às variações de $K_{i m-S}$, entanto que o modo $\lambda_{8,9}$ apresenta uma forte sensibilidade negativa ao incremento de $K_{i m-S}$, produzindo-se uma redução significativa do seu $\zeta$. Este comportamento mostra que, embora um reestabelecimento rápido da frequência ao seu valor programado seja desejado, a atuação do controle secundário de frequência unicamente depois de passado o transitório eletromecânico é essencial para manter a estabilidade dinâmica do sistema.

\subsubsection{Carga dinâmica - Motor de Indução}

Até aqui se tem estudado o comportamento dinâmico dos GGDs na presença de cargas passivas R-L. A seguir será analisada a estabilidade dinâmica dos GGDs quando cargas dinâmicas (i.e., motores de indução) são ligadas diretamente nos terminais do gerador. 
As características dos motores de indução (MI) utilizados nesta análise se mostram na Tabela 4.7 [54], onde todos os parâmetros são referidos ao estator. As potências nominais dos Mls foram propositalmente escolhidas inferiores às potências nominais dos GGDs, de forma a analisar a estabilidade dinâmica do sistema frente a sobrecargas dos motores.

Tabela 4.7 - Parâmetros dos Motores de Indução

\begin{tabular}{ccc}
\hline Parâmetro & MI1 & MI2 \\
\hline $\boldsymbol{P}[\boldsymbol{k W}] / \boldsymbol{f}[\boldsymbol{H z}]$ & $15 / 60$ & $30 / 60$ \\
$\boldsymbol{R}_{\boldsymbol{s}} / \boldsymbol{R}_{\boldsymbol{r}}[\boldsymbol{p u}]$ & $0.019 / 0.012$ & $0.018 / 0.010$ \\
$\boldsymbol{L}_{\boldsymbol{s s}} / \boldsymbol{L}_{\boldsymbol{r r}}[\boldsymbol{p u}]$ & $2.08 / 2.08$ & $2.07 / 2.07$ \\
$\boldsymbol{L}_{\boldsymbol{m}}[\boldsymbol{p u}]$ & 2.024 & 2.019 \\
$\boldsymbol{H}_{\boldsymbol{A S}}[\boldsymbol{s}] / \boldsymbol{K}_{\boldsymbol{A S D}}[\boldsymbol{p u}]$ & $0.12 / 0.03$ & $0.19 / 0.02$ \\
\hline
\end{tabular}

A Tabela 4.8 mostra os autovalores do sistema GGD+MI, para operação prédistúrbio nominal dos motores (i.e., torque mecânico nominal aplicado aos eixos dos motores). O sistema é descrito por doze variáveis de estado associadas aos GGDs e cinco variáveis de estado associadas aos Mls, consequentemente, há dezessete autovalores para cada caso.

Como pode se observar, com exceção do $\lambda_{17}$, todos os autovalores tem parte real negativa, indicando uma condição de estabilidade para o ponto de operação considerado. $\mathrm{O} \lambda_{17}=0$, está associado com a falta de uma referência absoluta para a medição do ângulo do rotor do GGD como explicado anteriormente.

A Figura 4.15 mostra os principais autovalores dos sistemas plotados no plano complexo. De forma geral observa-se que a disposição dos autovalores no plano complexo novamente é muito similar para os dois sistemas em estudo, entretanto, diferentemente do cenário com carga passiva, neste caso todos os modos oscilatórios do sistema apresentam $\zeta<0.4$. O caso mais crítico se registra para o $\lambda_{11,12}$, com $\zeta$ em torno de 0.09 e $w_{n}$ na faixa de $2.0 \mathrm{~Hz}$. 
Tabela 4.8 - Autovalores do Sistema - GGD + MI

\begin{tabular}{ccc}
\hline Autovalor & GGD1+MI1 & GGD2+MI2 \\
\hline 1 & $-2.01 \mathrm{e} 6$ & $-4.13 \mathrm{e} 6$ \\
2 & $-1.72 \mathrm{e} 6$ & $-3.57 \mathrm{e} 6$ \\
3,4 & $-74.48 \pm \mathrm{j} 368.00$ & $-82.51 \pm \mathrm{j} 367.46$ \\
5,6 & $-20.92 \pm \mathrm{j} 94.11$ & $-19.52 \pm \mathrm{j} 76.74$ \\
7 & -72.36 & -71.89 \\
8 & -35.69 & -33.66 \\
9 & -32.68 & -33.02 \\
10 & -17.10 & -20.11 \\
11,12 & $-1.12 \pm \mathrm{j} 13.08$ & $-1.198 \pm \mathrm{j} 13 \mathrm{i} .00$ \\
13,14 & $-1.99 \pm \mathrm{j} 7.97$. & $-1.65 \pm \mathrm{j} 7.91$ \\
15 & -8.07 & -9.49 \\
16 & -0.411 & -0.411 \\
17 & 0 & 0 \\
\hline
\end{tabular}
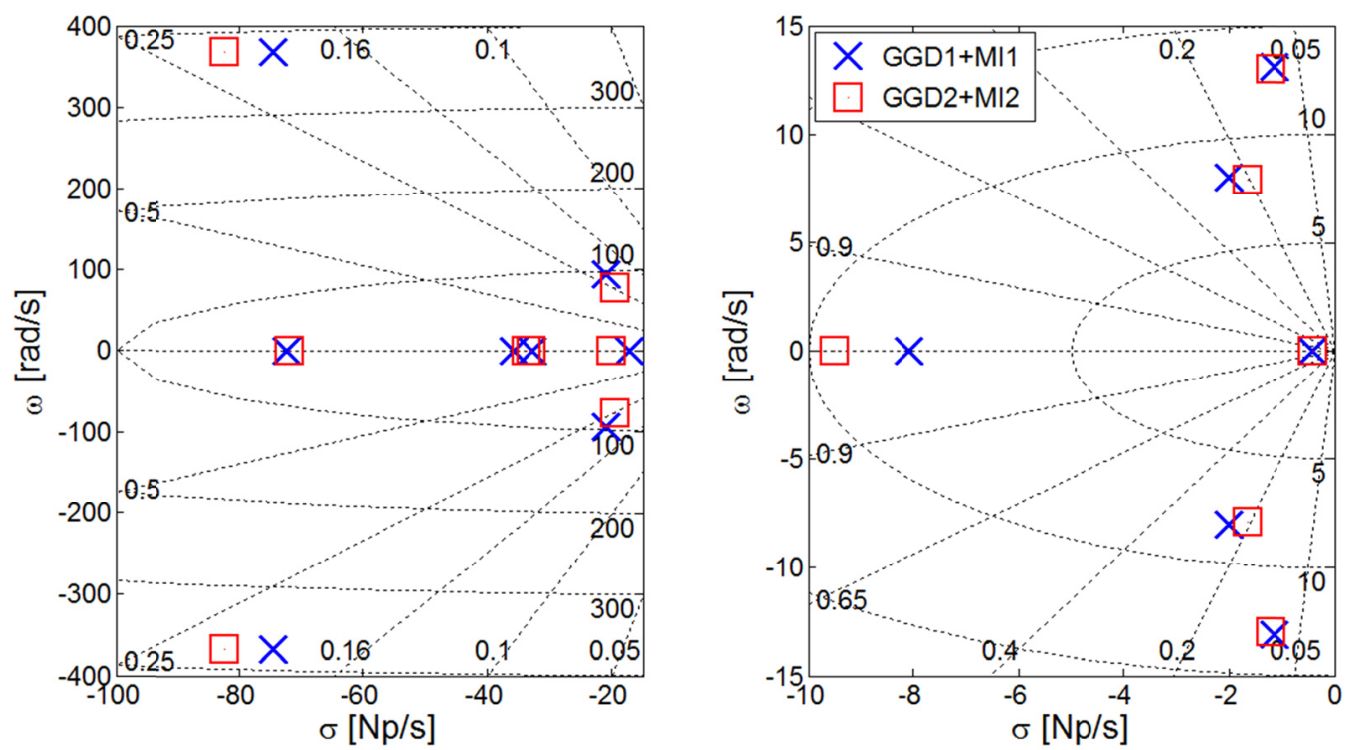

Figura 4.15 - Autovalores do Sistema - GGD + MI

Para uma melhor análise, foram calculados os fatores de participação das variáveis de estado para o primeiro sistema (GGD1+MI1). O resultado se mostra na Figura 4.16, onde os fatores de participação com valor inferior a 0.1 foram suprimidos por conveniência. Um gráfico similar pode ser obtido para o segundo sistema (GGD2+MI2). 


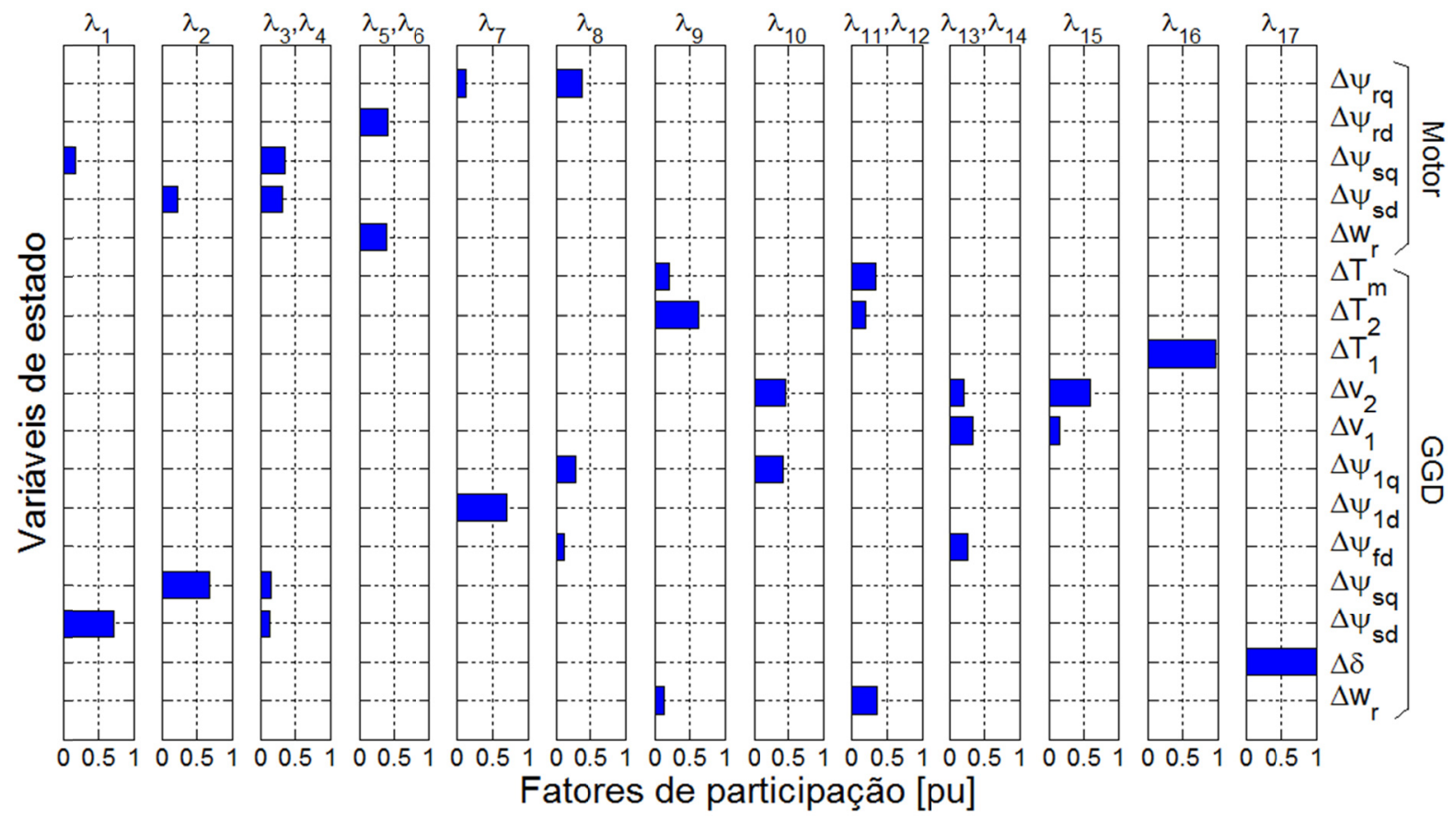

Figura 4.16 - Fatores de Participação - GGD1+|MI1

Observa-se que o primeiro par de autovalores oscilatórios $\left(\lambda_{3,4}\right)$ está relacionado com os enlaces de fluxo do estator do motor, consequentemente com as correntes transitórias em CC presentes nos enrolamentos do estator do motor. Este modo tem frequência fixa próxima aos $60 \mathrm{~Hz}$, sendo mais amortecido para o segundo sistema. Pelo contrário, o segundo modo oscilatório $\lambda_{5,6}$ está relacionado com o transitório elétrico presente no rotor do motor. $A$ frequência deste modo, conhecido como autovalor do rotor, é variável e muda em função do escorregamento da máquina.

O modo real $\lambda_{8}$ está associado principalmente aos enlaces de fluxo do eixo de quadratura do rotor do motor, consequentemente ao torque do motor. Um valor negativo deste modo indica a operação da máquina na seção da curva torque $\times$ velocidade com derivada negativa i.e., onde o funcionamento do motor é estável. Um valor positivo indica a operação da máquina na seção da curva com derivada positiva, i.e., onde o funcionamento do motor é instável (situação de stall do motor). Já os modos $\lambda_{11,12} e \lambda_{13,14}$ são os modos eletromecânicos de baixa frequência associados ao GGD.

Para analisar o impacto da sobrecarga do MI na dinâmica do sistema, uma análise de sensibilidade dos autovalores ao torque mecânico aplicado ao motor foi realizada. 
A Figura 4.17 mostra o lugar geométrico dos modos dominantes para variações no valor pré-distúrbio do torque entre $100 \%$ e $225 \%$, em passos de $25 \%$.

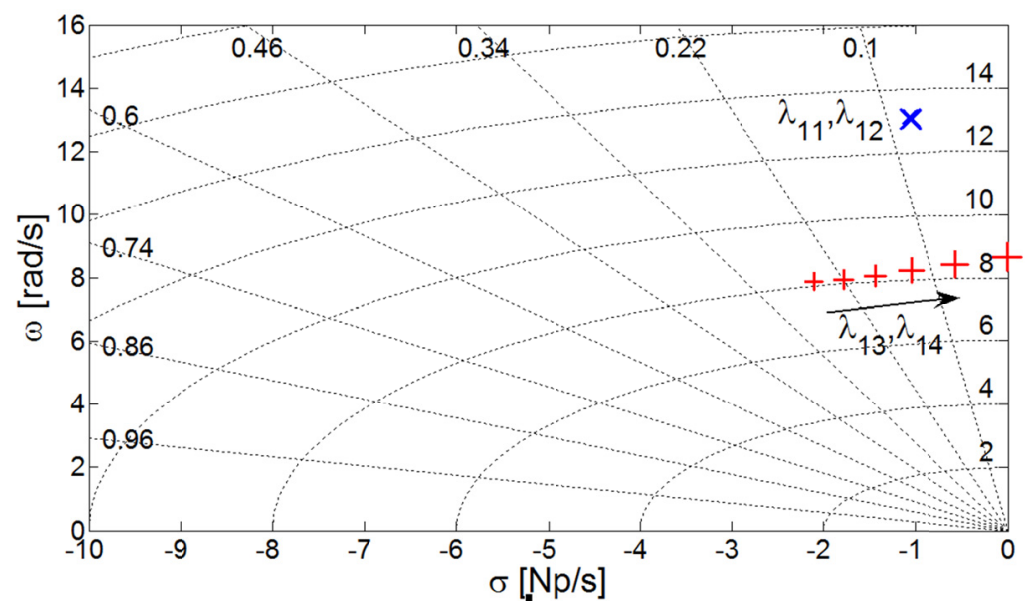

Figura 4.17 - Lugar geométrico do $\lambda_{11,12}$ e $\lambda_{13,14}$ para variações no Torque

A análise da Figura 4.17 mostra uma alta sensibilidade negativa do modo eletromecânico $\lambda_{13,14}$ (associado ao GGD) ao incremento do torque, com migração para a direita do plano complexo. Para este caso, um valor pré-distúrbio de torque mecânico aplicado ao motor de $225 \%$ acima do nominal, deixa o sistema marginalmente estável.

Já o modo eletromecânico $\lambda_{11,12}$ é praticamente insensível às variações no torque. Observa-se que neste caso, a perda de estabilidade do sistema ocorre por perda de sincronismo do GGD e não por stall do motor de indução.

Para corroborar os dados obtidos através da análise modal, simulações na plataforma Simulink® foram realizadas com modelos não lineares detalhados do sistema. Os resultados das simulações no domínio do tempo foram então comparados com os correspondentes resultados obtidos do modelo linear. A Figura 4.18 mostra as respostas no domínio do tempo de algumas das grandezas do sistema para um incremento súbito de $15 \%$ no torque mecânico aplicado ao eixo do motor, para condição pré-distúrbio nominal. 

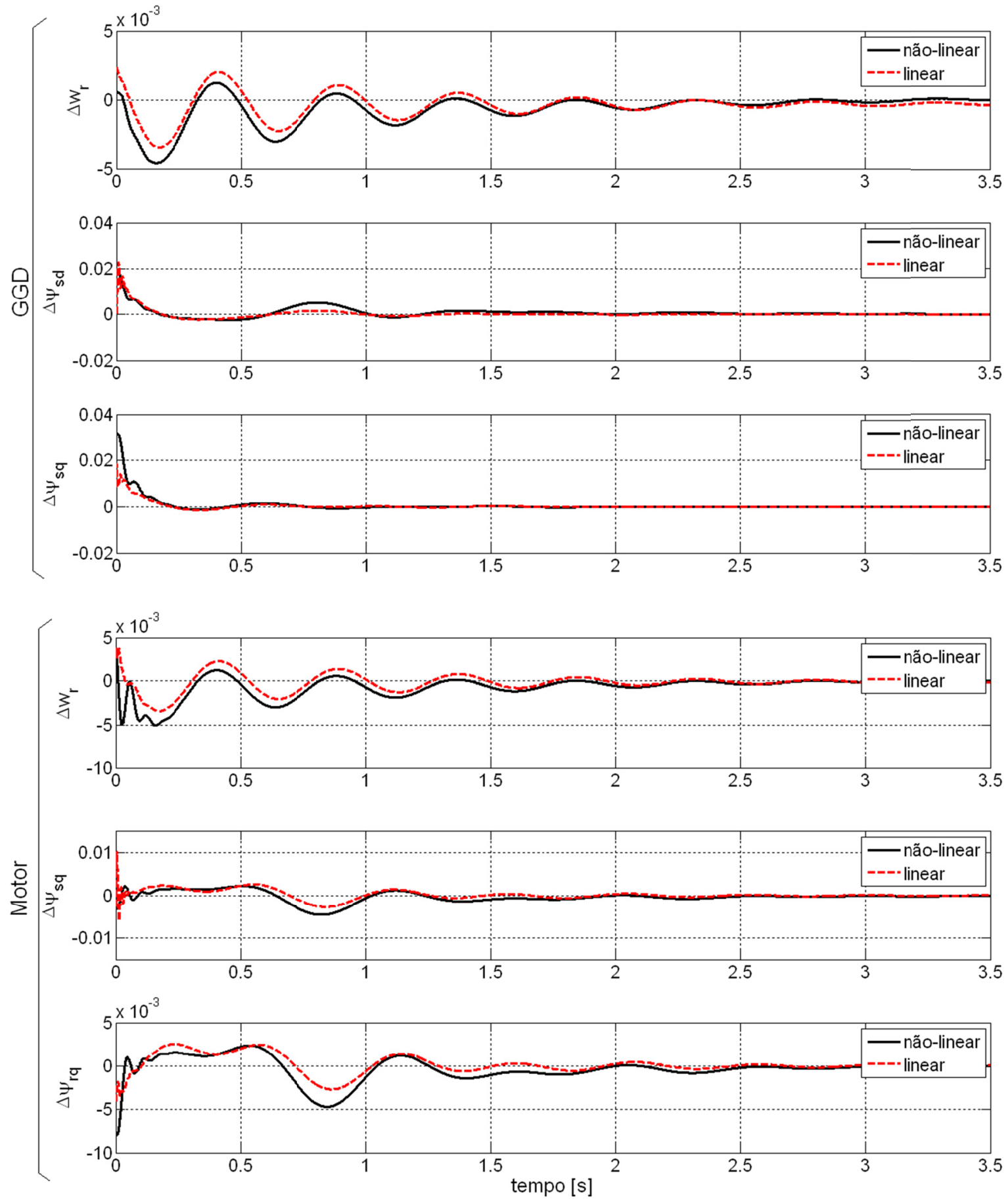

Figura 4.18 - Resposta transitória para 15\% de variação no Torque - GGD1+MI1

A comparação dos resultados obtidos através do modelo não linear com os resultados obtidos do modelo linear indica um padrão semelhante de comportamento das variáveis do sistema, com máximo sobrepasso (overshoot), tempo de assentamento (settling time) e constantes de tempo similares para os dois casos, até mesmo para valores elevados de distúrbio (15\%). 


\subsection{Inversores}

Antes de realizar o ajuste das malhas de controle dos inversores é de importância conhecer os parâmetros elétricos relacionados à interface do inversor com a rede. Infelizmente, estes parâmetros não são fornecidos pelos fabricantes de inversores comerciais, sendo necessário estimar seus valores a partir das taxas de distorção harmônica (THD) máximas na corrente e tensão de saída do inversor, fornecidas tanto pelo fabricante como recomendadas em normas.

Neste trabalho, um algoritmo de otimização para o projeto da interface dos inversores foi desenvolvido no ambiente Matlab/Simulink ${ }$. Este algoritmo visa atender tanto aos requisitos de qualidade de energia (especificamente no referente à distorção harmônica), quanto ao desempenho dinâmico do inversor. A metodologia proposta a seguir é composta de várias etapas, sendo algumas delas baseadas em [55].

\subsubsection{Projeto da Interface do Inversor com a rede}

Em se tratando do projeto de filtros passivos para inversores que sintetizam tensões senoidais através de modulação por largura de pulso (PWM) em frequências de chaveamento na ordem de dezenas de $\mathrm{kHz}$, as metodologias comumente encontradas na literatura especializada visam determinar os parâmetros do filtro que assegurem o atendimento à máxima THD admissível na tensão e corrente de saída do inversor, a um custo razoável dos elementos L-C. Já para os inversores que operam em frequências de chaveamento superiores, cuidados adicionais devem ser tomados para que o filtro também atenda às especificações com relação aos índices máximos aceitáveis de interferência eletromagnética. Os inversores comerciais que servem de interface para as FEDs que não geram naturalmente em 50 ou $60 \mathrm{~Hz}$, geralmente operam em frequências de chaveamento na ordem de dezenas de $\mathrm{kHz}$, assim sendo, este trabalho não entra no mérito com relação à interferência eletromagnética. 
O projeto da interface do inversor (i.e., filtro LC + indutor de acoplamento) pode ser abordado como o projeto de um filtro $\mathrm{LCL}$, tirando vantagem da maior flexibilidade associada a esta configuração, que em geral, resulta em elementos L-C mais leves. Todavia, especial atenção deve ser dada ao cálculo do indutor de acoplamento, pois deste elemento depende a regulação de potência e tensão no ponto de conexão do inversor com a rede.

O ponto de partida do algoritmo é a característica potência-ângulo existente entre o inversor e a rede. Tomando como referência a Figura 4.19, a potência ativa $(P)$ transferida do inversor para a rede (desprezando o valor de $R_{c}$ ) pode ser descrita pela eq. (4.3).

$$
P=\frac{v_{c} \cdot v_{b}}{w \cdot L_{c}} \cdot \operatorname{sen}(\theta)
$$

Como mostra a eq. (4.3), o controle do fluxo de potência ativa é realizado através do controle do ângulo $\theta$ sintetizado pelo inversor na saída do filtro LC. A faixa de operação de $\theta$ está entre $0^{\circ}$ (para injeção de potência nula), até $\theta_{M A X}$ (para injeção de potência máxima).

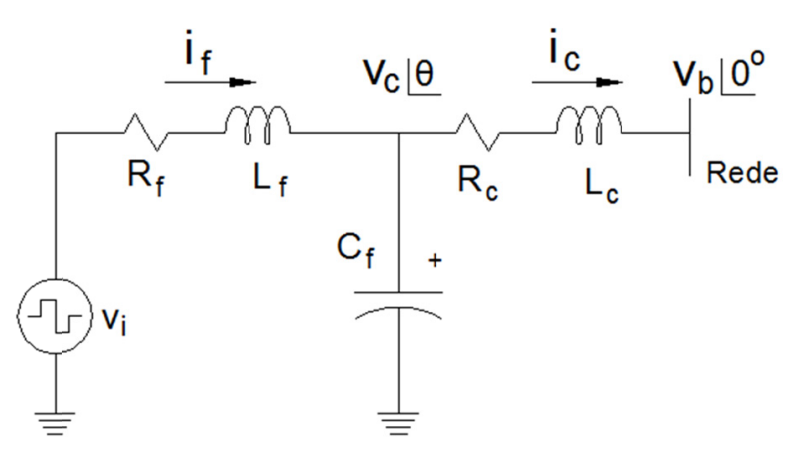

Figura 4.19 - Interface do Inversor - Filtro LC + Indutor de acoplamento

Para garantir que o controle trabalhe na parte linear da curva $P=f(\theta)$, o valor de $\theta$ dever ser limitado, para toda a faixa de operações do inversor, à região onde a curva "sen $(\theta)$ " é praticamente linear, sendo um valor de $\theta_{M A X}$ inferior a $10^{\circ}$ uma escolha 
razoável. Desta condição, deriva-se que o valor máximo de $L_{c}$ é dado pela seguinte equação:

$$
L_{c-M A X}=\frac{v_{c} \cdot v_{b}}{w \cdot P_{M A X}} \cdot \operatorname{sen}\left(10^{\circ}\right)
$$

Por outra parte, valores muito baixos de $L_{c}$ também devem ser evitados, pois conduzem a um $\theta_{\text {MAX }}$ muito pequeno, deixando a faixa de operação de $\theta$ muito estreita, requerendo maior precisão, por parte do controle do inversor, na sintetização do ângulo. Faixas de operação de $\theta$ inferiores a $1^{\circ}$ devem ser evitadas. Desta condição deriva-se o valor mínimo de $L_{c}$ dado pela eq. (4.5):

$$
L_{c-M I N}=\frac{v_{c} \cdot v_{b}}{w_{n} \cdot P_{M A X}} \cdot \operatorname{sen}\left(1^{\circ}\right)
$$

Partindo do valor de $L_{c-M A X}$, o algoritmo de otimização visa maximizar o fator de atenuação da corrente $i_{c}$ com relação à corrente $i_{f}$ na frequência de chaveamento. Este fator corresponde à função custo $(F C)$ do algoritmo e pode ser estimado através da seguinte expressão [55]:

$$
F C=\frac{i_{c}\left(h_{s w}\right)}{i_{f}\left(h_{s w}\right)} \approx \frac{Z_{L C}^{2}}{\left|w_{r e s}^{2}-w_{s w}^{2}\right|}
$$

Com:

$$
Z_{L C}=\sqrt{\frac{1}{L_{c} \cdot C_{f}}} \quad w_{\text {res }}=\sqrt{\frac{L_{c}+L_{f}}{L_{c} \cdot L_{f} \cdot C_{f}}}
$$

Onde, 
$i_{c}\left(h_{s w}\right) e i_{f}\left(h_{s w}\right)$ componente de corrente na frequência de chaveamento [A].

$w_{s w} \quad$ frequência de chaveamento do inversor $[\mathrm{rad} / \mathrm{s}]$.

$w_{\text {res }} \quad$ frequência de ressonância do filtro $[\mathrm{rad} / \mathrm{s}]$.

A maximização da FC deve atender as seguintes restrições:

a) A $w_{\text {res }}$ deve ser limitada a uma faixa entre dez vezes a frequência nominal $\left(w_{n}\right)$ do sistema e uma fração da $w_{s w}$, (usualmente 0.5 ), de modo a não criar problemas de ressonância na parte inferior e superior do espectro harmônico, e ao mesmo tempo, garantir uma adequada atenuação da $w_{s w}$.

b) A indutância total da interface $\left(L_{f}+L_{c}\right)$ deve ser menor que $10 \%$ da indutância base do sistema, calculada sobre os valores nominais de potência e tensão do inversor, de forma a limitar a queda de tensão na interface.

c) O valor máximo de $C_{f}$ é limitado pela máxima degradação do fator de potência na saída do filtro, que pode resultar em perda do controle da tensão devido a uma elevada capacitância, especialmente na condição de carga leve. Uma injeção de potência reativa inferior a $5 \%$ é tolerável.

Se alguma das restrições acima mencionadas não puder ser satisfeita para o valor inicial de $L_{c}=L_{c-M A X}$, O algoritmo diminui o valor de $L_{c}$ num pequeno valor préespecificado $\left(\Delta L_{c}\right)$, começando novamente o processo de otimização até satisfazer todas as restrições. Se o valor de $L_{c-M I N}$ é atingido, nenhuma solução satisfatória pode ser encontrada. Pelo contrário, se todas as restrições são atendidas, o valor de THD de tensão e corrente na saída do filtro é determinado via simulação computacional no domínio do tempo no ambiente Simulink®, através de modelos não lineares detalhados do inversor. Provavelmente, nesta etapa, o valor de THD já esteja dentro dos limites especificados, caso contrário, a relação entre a $w_{r e s}$ e $w_{s w}$ é diminuída e retorna-se novamente ao processo de otimização de $L_{f} e C_{f}$. Para os casos onde o THD não é especificado pelo fabricante, faz-se uso da Norma IEEE 929-2000, Norma IEC 61727-2004 e Norma IEC 62040-3, cujos limites de distorção harmônica são reproduzidos na Tabela 4.9 e Tabela 4.10 por conveniência. 
Tabela 4.9 - Limites de distorção de corrente [56] [57]

\begin{tabular}{cc}
\hline Harmônicas ímpares & Limite da Distorção \\
\hline $3^{\text {rd }}-9^{\text {th }}$ & $<4.0 \%$ \\
$11^{\text {th }}-15^{\text {th }}$ & $<2.0 \%$ \\
$17^{\text {th }}-21^{\text {st }}$ & $<1.5 \%$ \\
$23^{\text {rd }}-33^{\text {rd }}$ & $<0.6 \%$ \\
Acima da $33^{\text {rd }}$ & $<0.3 \%$ \\
\hline Harmônicas pares & Limite da Distorção \\
$2^{\text {rd }}-8^{\text {th }}$ & $<1.0 \%$ \\
$10^{\text {th }}-32^{\text {nd }}$ & $<0.5 \%$ \\
\hline
\end{tabular}

Tabela 4.10 - Limites de distorção de tensão [58]

\begin{tabular}{cccc}
\hline Harmônicas & $\begin{array}{c}\text { Limite da } \\
\text { Distorção }\end{array}$ & Harmônicas & $\begin{array}{c}\text { Limite da } \\
\text { Distorção }\end{array}$ \\
\hline $3^{\text {rd }}$ & $<5.0 \%$ & $15^{\text {th }}$ & $<0.3 \%$ \\
$5^{\text {th }}$ & $<6.0 \%$ & $21^{\text {th }}$ & $<0.2 \%$ \\
$7^{\text {th }}$ & $<5.0 \%$ & $2^{\text {rd }}$ & $<2.0 \%$ \\
$9^{\text {th }}$ & $<1.5 \%$ & $4^{\text {th }}$ & $<1.0 \%$ \\
$11^{\text {th }}$ & $<3.5 \%$ & $6^{\text {th }}$ & $<0.5 \%$ \\
$13^{\text {th }}$ & $<3 \%$ & $8^{\text {th }}$ & $<0.5 \%$ \\
\hline
\end{tabular}

A Figura 4.20 mostra o fluxograma da metodologia proposta para o dimensionamento da interface do inversor com a rede.

\subsubsection{Controle Droop}

\subsubsection{Ajuste das malhas de controle}

De forma similar ao caso dos GGDs, para o ajuste das malhas de controle do Inversor Droop é de interesse conhecer as especificações da resposta transitória que o inversor deve apresentar segundo Normas. Embora existam várias normas internacionais que abordem especificamente o tema relacionado aos requisitos mínimos que FEDs devem atender para operação autônoma ou em paralelo com a 
rede convencional [56] [57] [59] [60], nenhuma delas estabelece índices de desempenho para a resposta dinâmica dos mesmos. Todavia, existem normas referentes a Fontes Ininterruptas de Energia (UPS) que podem auxiliar nesta tarefa.

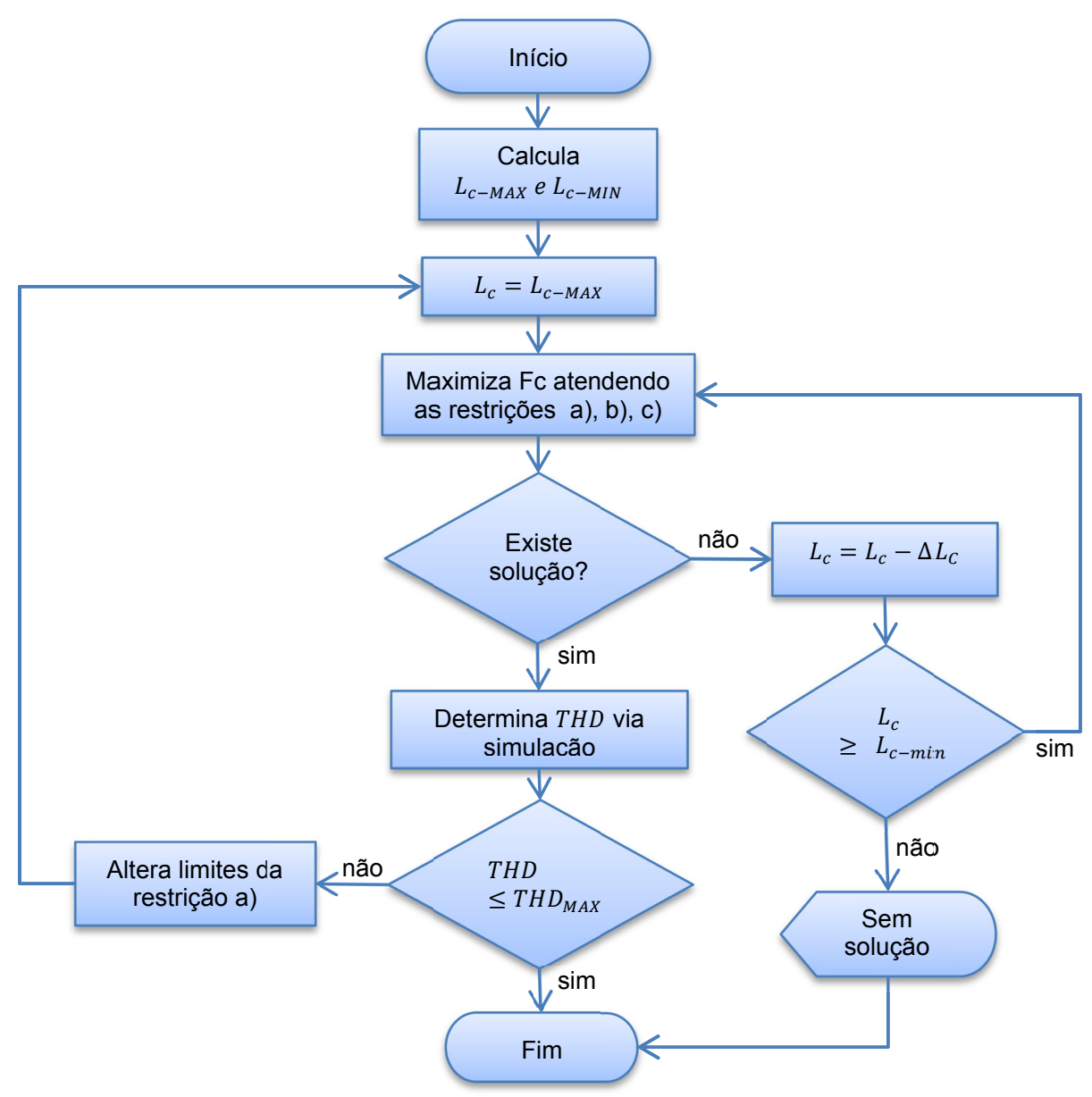

Figura 4.20 - Fluxograma para otimização da interface do Inversor

Neste trabalho se utiliza especificamente a Norma IEC 62040-3 como referência para o ajuste das malhas de controle. Esta norma divide as UPS's em três grandes grupos: passive standby, line-interactive e double conversion, sendo que o Inversor com Controle Droop $\left(I N V_{D}\right)$ se enquadra melhor no último grupo. Os limites da resposta dinâmica de tensão, para um degrau de 100\% de rejeilção de carga linear, se mostram na Figura 4.21 [58]. 


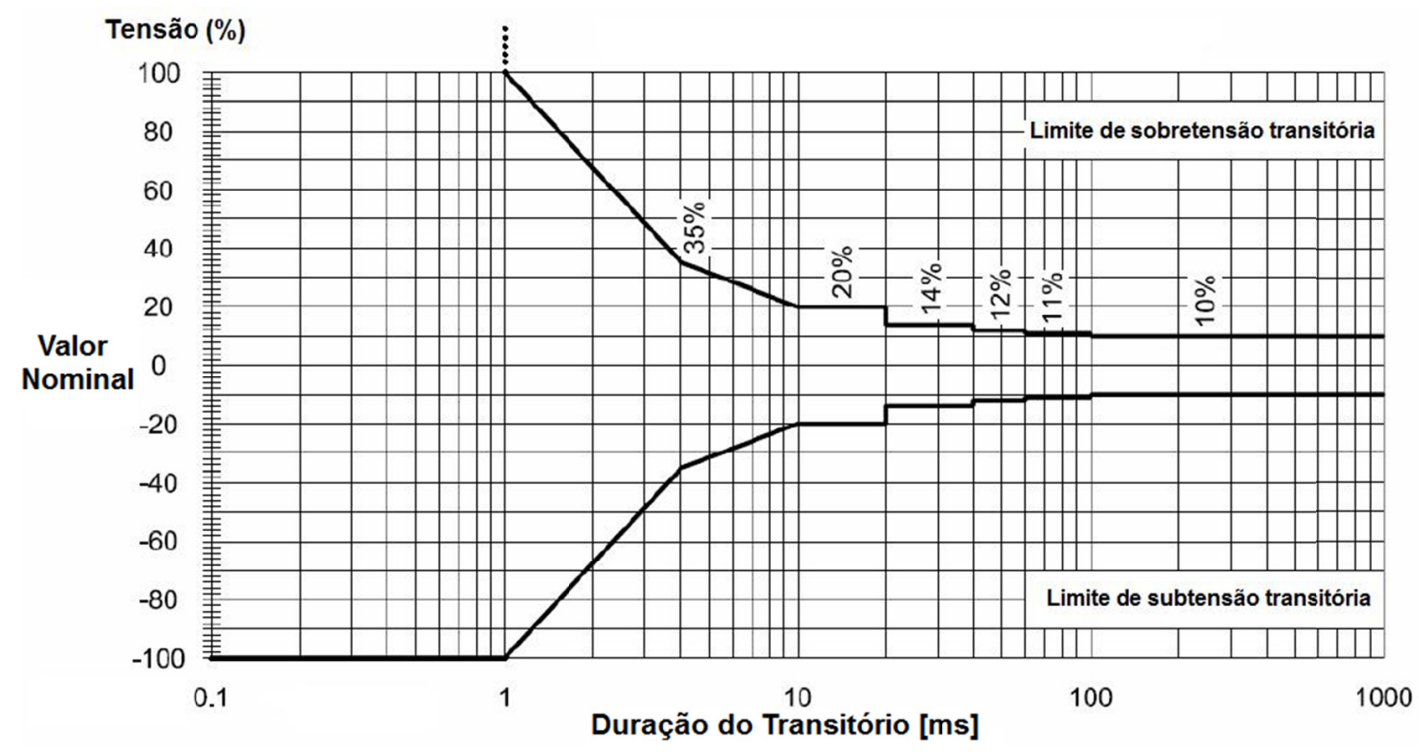

Figura 4.21 - Resposta transitória de tensão - Inversor Droop

As potências de inversores formadores de rede disponíveis no mercado para aplicações em MGs estão na faixa de unidades a dezenas de kW. Assim sendo, neste trabalho analisaremos dois inversores trifásicos com potências nominais de 15 kW e 30 kW, com as características elétricas mostradas na Tabela 4.11 [61]

Tabela 4.11 - Parâmetros dos Inversores - Controle Droop

\begin{tabular}{ccc}
\hline Parâmetro & $\boldsymbol{I N V \mathbf { 1 } _ { \boldsymbol { D } }}$ & $\boldsymbol{I N V \boldsymbol { 2 } _ { \boldsymbol { D } }}$ \\
\hline $\mathrm{P}[\mathrm{kW}] / \mathrm{fp}$ & $15 / 0.8$ & $30 / 0.8$ \\
Tensão $[\mathrm{V}]$ & $220 / 127$ & $220 / 127$ \\
$\boldsymbol{f}[\boldsymbol{H z}] / \boldsymbol{w}_{\boldsymbol{s w}}[\boldsymbol{k H z}]$ & $60 / 10$ & $60 / 10$ \\
Droop freq./tens.[\%] & $0.83 / 5$ & $0.83 / 5$ \\
No. Pulsos & 6 & 6 \\
THD corrente & $<3 \%$ & $<3 \%$ \\
THD tensão & $<5 \%$ & $<5 \%$ \\
\hline
\end{tabular}

Para o ajuste das malhas de controle dos inversores se utiliza uma abordagem diferente da empregada nos GGDs. Neste caso, ajustes preliminares para os ganhos das malhas de controle são obtidos através do enfoque clássico de polos e zeros e resposta em frequência do sistema. Para isto, funções de transferência para cada malha de controle são derivadas a partir do modelo no espaço de estados mostrado 
no Capítulo 3. A largura de banda da malha interna (malha de corrente) é determinada em função da frequência de chaveamento do inversor, de forma a garantir uma boa rejeição das perturbações de alta frequência. Para um valor de $w_{s w}=10 \mathrm{kHz}$, uma largura de banda na faixa de $1.5 \mathrm{kHz}$ apresenta um bom compromisso custo-benefício para inversores de médio e grande porte [35].

Tratando-se de um controle em cascata, o recomendável é projetar cada malha de controle com uma largura de banda entre três e cinco vezes menor que a malha de controle interna imediata. Desta forma, a malha de tensão é projetada com uma largura de banda na faixa de $400 \mathrm{~Hz}$. Já a malha de controle de potência é projetada com uma largura de banda muito mais baixa (em torno de $10 \mathrm{~Hz}$ ) para garantir uma adequada extração das componentes fundamentais de $\mathrm{P}$ e $\mathrm{Q}$ a partir das medições instantâneas de tensão e corrente na saída do inversor ${ }^{16}$ [35]. Para cada uma das malhas, a margem de fase é mantida entre $40^{\circ}$ e $60^{\circ}$, para garantir um adequado amortecimento da resposta transitória [31]. Após se obter um desempenho dinâmico aceitável do sistema, ajustes finos são realizados nos ganhos dos controladores baseados na análise dos autovalores. Os ajustes são realizados com carga nominal $R-L, f p=0.8$, diretamente ligada aos terminais do inversor.

Os valores finais dos ganhos das malhas de controle, assim como dos parâmetros elétricos da interface do inversor (calculada aplicando-se a metodologia mostrada na Seção 4.2.1), se mostram na Tabela 4.12.

Tabela 4.12 - Ganhos das malhas de controle - Controle Droop

\begin{tabular}{ccc}
\hline Parâmetro & INV1 & INV2 \\
\hline $\boldsymbol{R}_{\boldsymbol{c}} / \boldsymbol{L}_{\boldsymbol{c}}[\boldsymbol{p u}]$ & $0.007 / 0.075$ & $0.007 / 0.072$ \\
$\boldsymbol{R}_{\boldsymbol{f}} / \boldsymbol{L}_{\boldsymbol{f}} / \boldsymbol{C}_{\boldsymbol{f}}[\boldsymbol{p u}]$ & $0.002 / 0.023 / 0.05$ & $0.002 / 0.026 / 0.04$ \\
$\boldsymbol{K}_{\boldsymbol{p} \mathbf{1 - D} \boldsymbol{D}} / \boldsymbol{K}_{\boldsymbol{i 1 - D}}[\boldsymbol{p u}]$ & $0.413 / 1476$ & $0.355 / 1145.8$ \\
$\boldsymbol{K}_{\boldsymbol{p 2 - \boldsymbol { D }}} / \boldsymbol{K}_{\boldsymbol{i 2 - \boldsymbol { D }}}[\boldsymbol{p u}]$ & $0.574 / 1512$ & $0.672 / 1770.6$ \\
$\boldsymbol{F}_{\boldsymbol{v}-\boldsymbol{D}} / \boldsymbol{F}_{\boldsymbol{c}-\boldsymbol{D}}$ & $0.95 / 0.8$ & $0.95 / 0.8$ \\
$\boldsymbol{w}_{\boldsymbol{c}}[\mathbf{r a d} / \boldsymbol{s}]$ & 40 & 40 \\
\hline
\end{tabular}

\footnotetext{
${ }^{16}$ Como será visto mais adiante, a largura de banda da malha de controle de potência está associada diretamente à frequência de corte do filtro passa baixa de primeira ordem.
} 
Para verificar se os ajustes realizados nas malhas de controle atendem às especificações da Norma 62040-3, uma rejeição de carga $(R-L-f p=0.8)$ de $100 \%$ foi simulada no domínio do tempo para o $I N V 1_{D}$ no ambiente Simulink ${ }^{\circledR}$, com modelos não lineares detalhados do inversor. O resultado se mostra na Figura 4.22, e como pode se observar, os ajustes das malhas de controle são satisfatórios. Um desempenho similar foi apresentado pelo $I N V 2_{D}$.

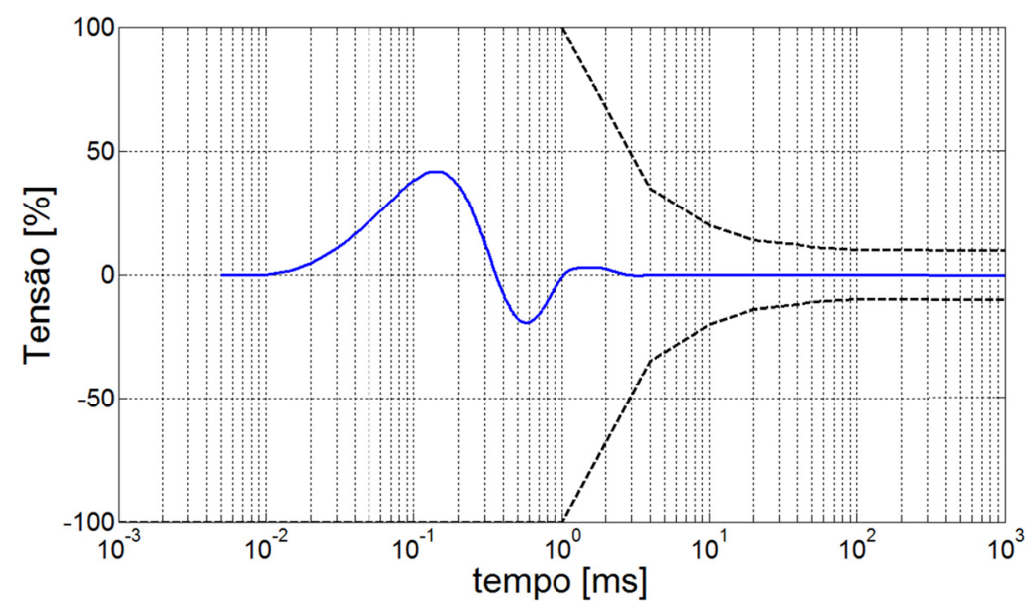

Figura 4.22 - Resposta transitória de tensão $I N V 1_{D}$ - Rejeição de carga

O espectro harmônico de tensão e corrente na carga (calculado através de simulação computacional) se mostra na Figura 4.23 e Figura 4.24 respectivamente para o $I N V 1_{D}$. A análise das figuras mostra que os valores de distorção harmônica individuais de corrente e tensão estão dentro dos limites apresentados na Tabela 4.9 e Tabela 4.10, tendo-se assim um indicativo de que o projeto do filtro é adequado. Valores similares foram obtidos para o $I N V 2_{D}$.

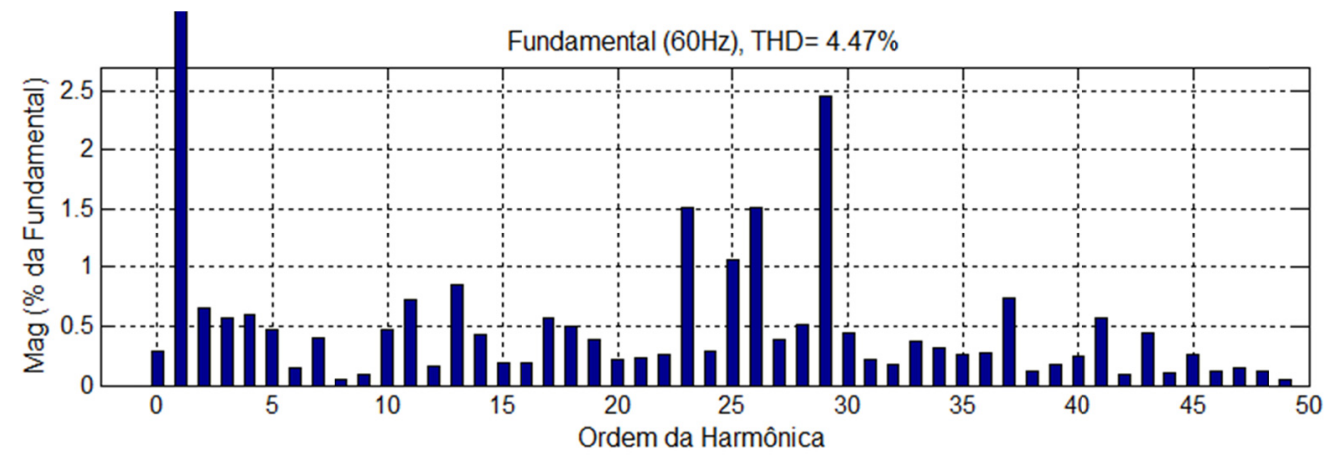

Figura 4.23 - Espectro Harmônico de Tensão - INV1 


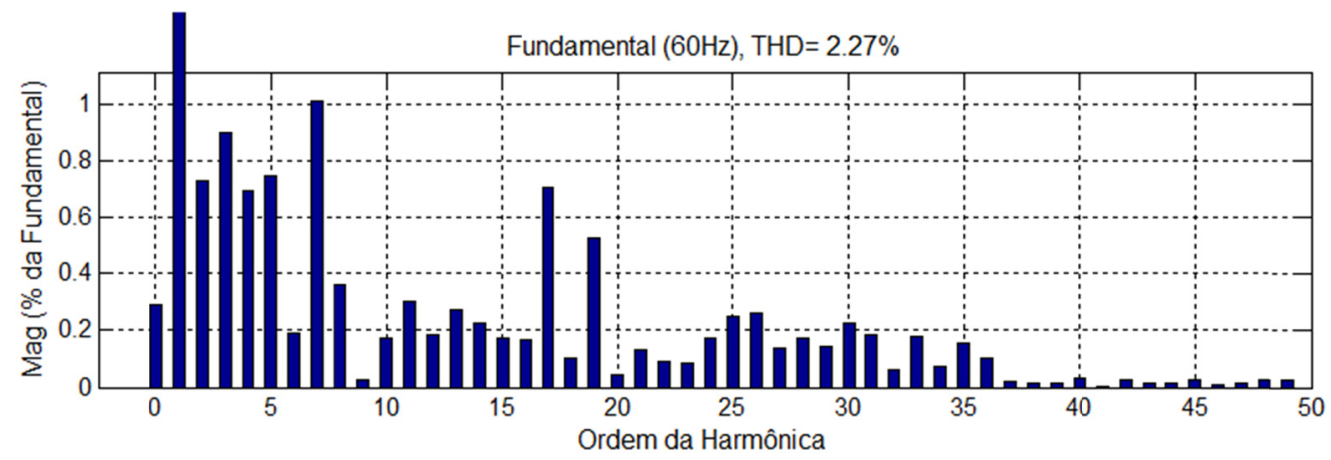

Figura 4.24 - Espectro Harmônico de Corrente - INV1

\subsubsection{Análise da estabilidade dinâmica}

Carga Passiva $-R-L$

Ajustadas as malhas de controle procede-se a analisar a estabilidade dinâmica dos Inversores através do enfoque dos autovalores da matriz de estado. A Tabela 4.13 mostra os autovalores correspondentes à operação pré-distúrbio nominal dos Inversores com carga $R-L, f p=0.8$, diretamente ligada aos seus terminais. O sistema é descrito por catorze variáveis de estado associadas aos modelos no espaço de estados dos Inversores e duas variáveis de estado associadas aos modelos no espaço de estados das cargas, consequentemente, tem-se dezesseis autovalores $(\lambda)$ para cada caso ${ }^{17}$.

Como pode se observar, com exceção do $\lambda_{15}$ e $\lambda_{16}$, todos os autovalores tem parte real negativa, indicando uma condição de estabilidade para o ponto de operação considerado. Os autovalores $\lambda_{15}$ e $\lambda_{16}$ estão associados à falta de uma referência absoluta para a medição do ângulo $\theta$ e ângulo $\delta$ respectivamente.

\footnotetext{
17 Deve-se lembrar que na modelagem no espaço de estados realizada neste trabalho, existe um resistor virtual $\left(R_{v}\right)$ de grande magnitude conectado entre cada nó da rede e a terra. Consequentemente, a corrente de saída do inversor $\left(i_{c}\right)$ e a corrente da carga $\left(i_{\text {carga }}\right)$ são diferentes, não existindo redundância destes estados no modelo.
} 
Tabela 4.13 - Autovalores do Sistema $-I N V_{D}+$ Carga nominal R-L

\begin{tabular}{ccc}
\hline Autovalor & $\boldsymbol{I N V \mathbf { 1 } _ { \boldsymbol { D } }}$ & $\boldsymbol{I N V}_{\boldsymbol{D}}$ \\
\hline 1,2 & $-9.12 \mathrm{e} 5 \pm \mathrm{j} 377.38$ & $-1.88 \mathrm{e} 6 \pm \mathrm{j} 377.36$ \\
3,4 & $-3707.1 \pm \mathrm{j} 7311.6$ & $-3895.6 \pm \mathrm{j} 8130.8$ \\
5,6 & $-3390.7 \pm \mathrm{j} 6891.9$ & $-3610.2 \pm \mathrm{j} 7709.4$ \\
7,8 & $-1237.3 \pm \mathrm{j} 1762.7$ & $-1194.7 \pm \mathrm{j} 1655.3$ \\
9,10 & $-1165.3 \pm \mathrm{j} 1735.9$ & $-1127.5 \pm \mathrm{j} 1630.8$ \\
11,12 & $-424.39 \pm \mathrm{j} 362.29$ & $-424.05 \pm \mathrm{j} 358.11$ \\
13 & -44.585 & -44.584 \\
14 & -39.708 & -39.707 \\
15 & 0 & 0 \\
16 & 0 & 0 \\
\hline
\end{tabular}

A Figura 4.25 mostra os principais autovalores dos sistemas plotados no plano complexo. De forma geral observa-se que a disposição dos autovalores é muito similar para os dois inversores em estudo.
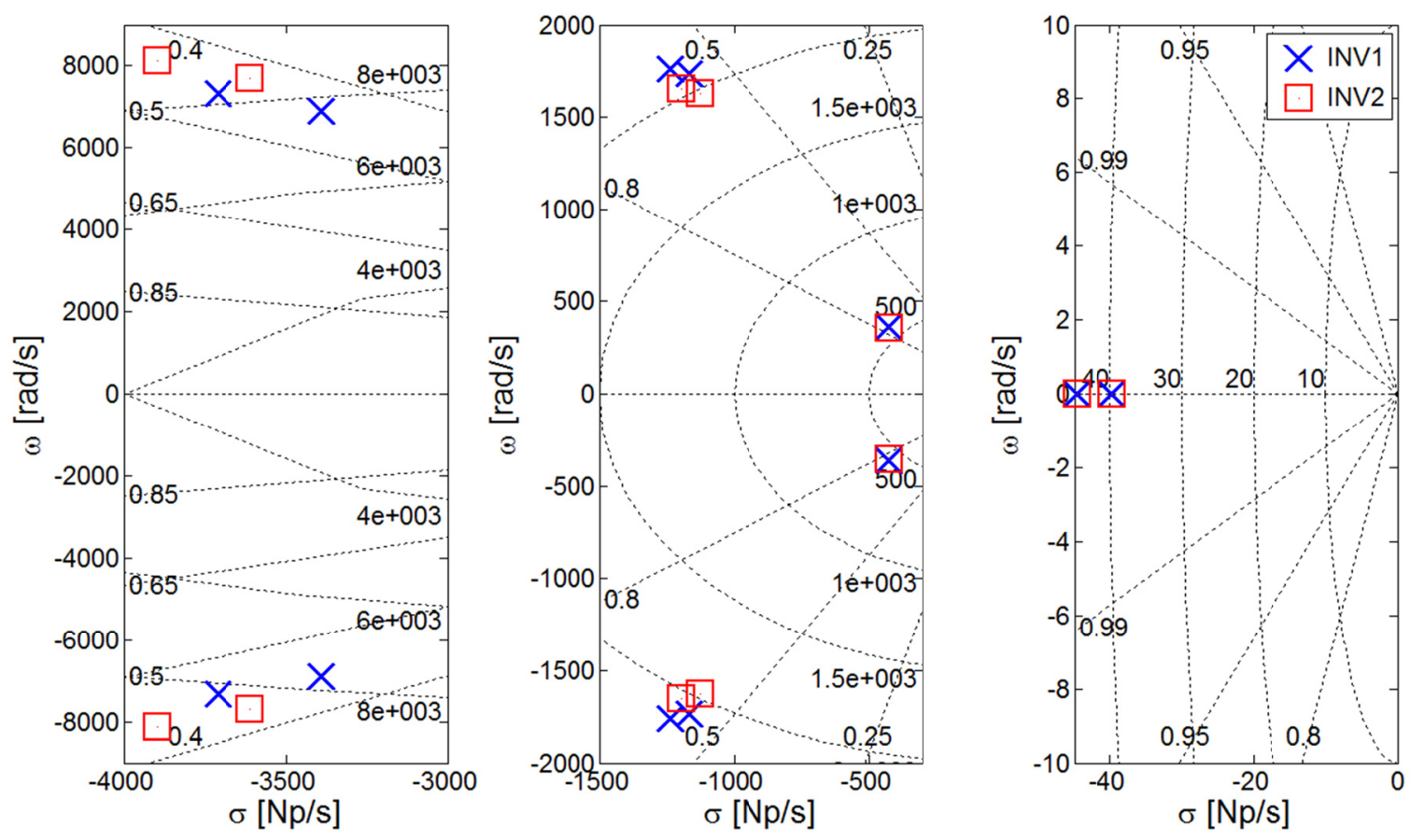

Figura 4.25 - Autovalores do Sistema - INV $\boldsymbol{D}_{D}+$ Carga R-L

Como esperado, observa-se que os autovalores estão localizados em três regiões de frequência bem definidas, correspondentes às larguras de banda utilizadas no 
projeto das três malhas de controle associadas ao Controle Droop. Por outra parte, todos os autovalores apresentam um elevado $\zeta(>0.4)$, indicando um adequado amortecimento da resposta transitória do sistema.

Para uma melhor análise foram calculados os fatores de participação das variáveis de estado nos modos do sistema. O resultado se mostra na Figura 4.26, onde os fatores de participação com valor inferior a 0.1 foram suprimidos por conveniência. A análise da Figura 4.26 mostra que os autovalores $\lambda_{1,2}$ e $\lambda_{11,12}$, com frequência amortecida em torno de $60 \mathrm{~Hz}$, estão relacionados diretamente com as correntes transitórias de CC presentes no indutor de acoplamento e na carga. Já os modos $\lambda_{3,4}$ e $\lambda_{5,6}$, com $w_{n}$ na faixa de $1.5 \mathrm{kHz}$, estão associados à malha de controle de corrente, enquanto os modos $\lambda_{7,8}$ e $\lambda_{9,10}$, com $w_{n}$ em torno de $400 \mathrm{~Hz}$, estão relacionados à malha de controle de tensão. Os autovalores $\lambda_{14}$ e $\lambda_{13}$ estão diretamente ligados ao controle de potência ativa e reativa respectivamente, com $\sigma$ na ordem da frequência de corte do FPB. É importante observar que na ausência de outras fontes no sistema que compartilhem a potência ativa e reativa demandada pela carga, estes modos são reais, i.e., não existem modos dominantes oscilatórios de baixa frequência para a operação individual do Inversor Droop com carga R-L, diferentemente do caso do GGD.

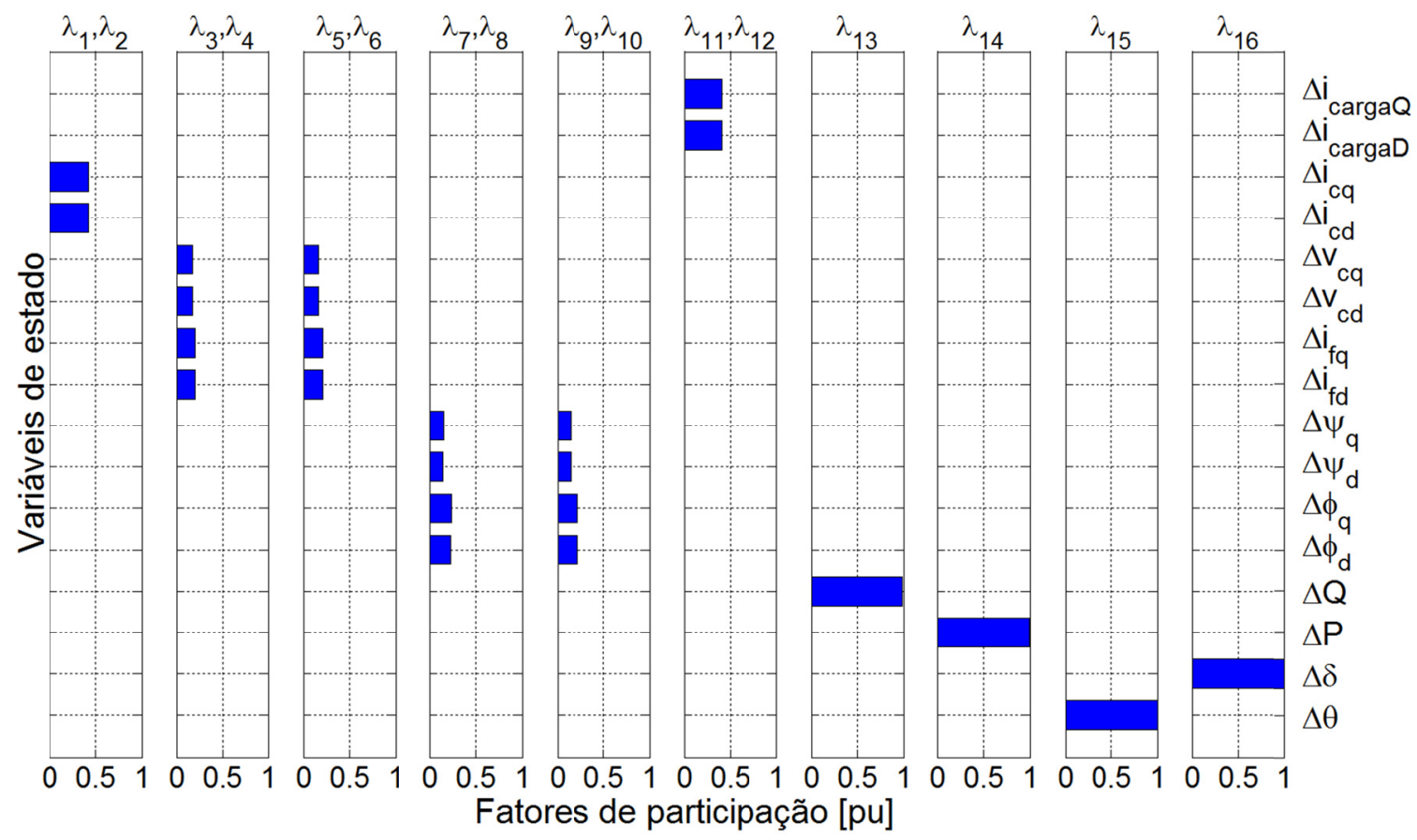

Figura 4.26 - Fatores de Participação - INV $1_{D}$ + Carga nominal R-L 
Observa-se também que não existe iteração da variável de estado $\Delta P$ no modo $\lambda_{13}$ nem da variável $\Delta Q$ no modo $\lambda_{14}$. Isto mostra que, se tratando de um sistema predominantemente indutivo, o controle de potência ativa e reativa é praticamente desacoplado. Todavia, em sistemas mais resistivos, observar-se ia uma interação de $\Delta P$ no $\lambda_{13}$ e de $\Delta Q$ no $\lambda_{14}$. Finalmente, como já explicado, o $\lambda_{15}$ e $\lambda_{16}$ estão relacionados à falta de uma referência absoluta para a medição do ângulo $\theta \mathrm{e}$ ângulo $\delta$ respectivamente.

Variações na potência ativa e fator de potência da carga alteram ligeiramente a posição dos autovalores do sistema. A Figura 4.27 mostra o llugar geométrico da parte real $(\sigma)$, imaginária $(w)$, fator de amortecimento $(\zeta)$, e frequência natural $\left(w_{n}\right)$ do par de modos que apresentam a maior sensibilidade $\left(\lambda_{11,12}\right)$, para variações na potência ativa $(P)$ e no fator de potência (fp) da condição pré-distúrbio do inversor. Como se pode observar, o $\lambda_{11,12}$ é positivamente sensível ao incremento do fp da carga, observando-se um incremento de $\zeta$ à medida que o fp aumenta.
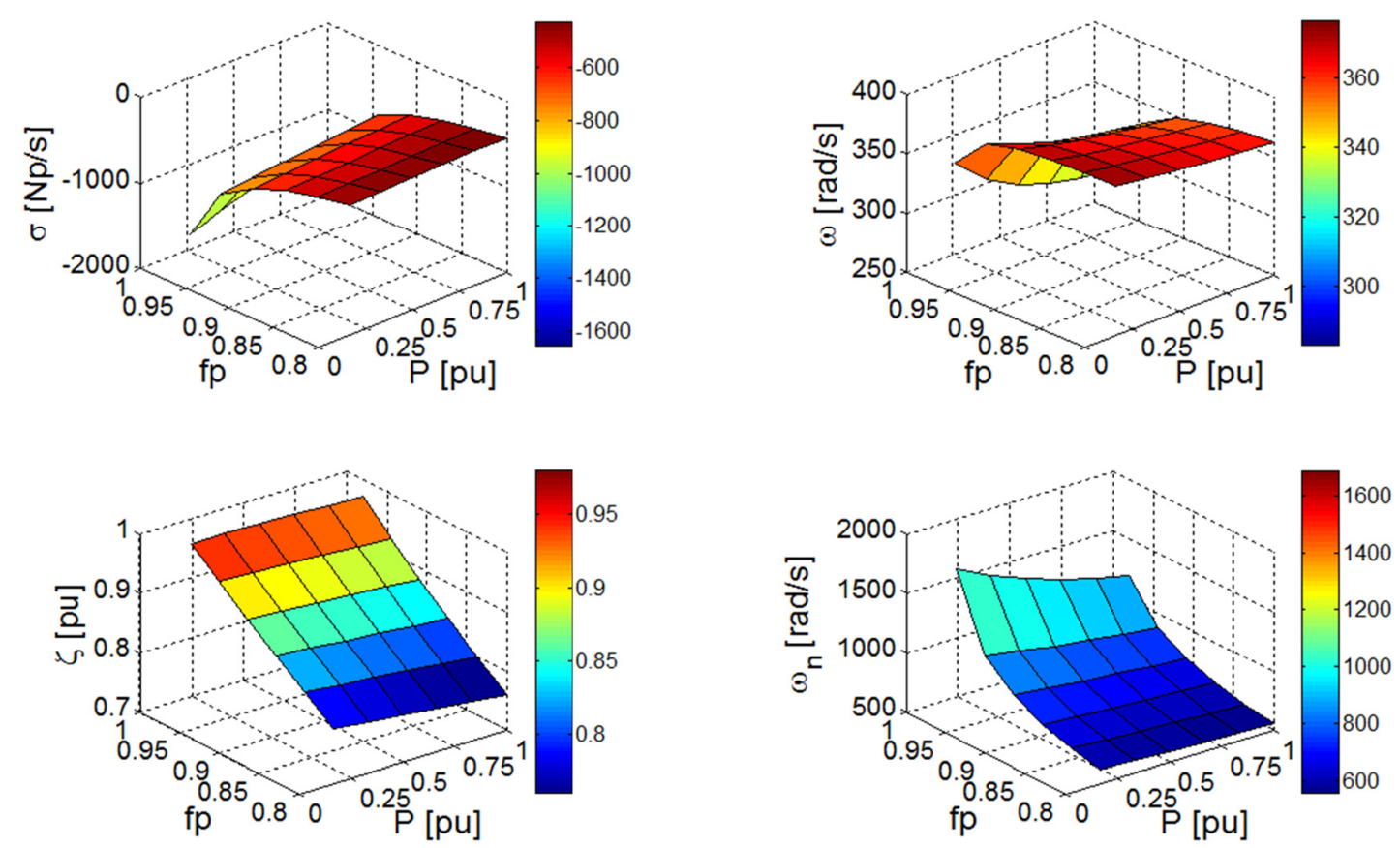

Figura 4.27 - Lugar geométrico do $\lambda_{11,12}$ para variações de P e fp - INV $1_{D}$

Para validar os dados obtidos através da análise modal, simulações na plataforma Simulink $®$ foram realizadas com modelos não lineares detalhados do sistema. Os 
resultados das simulações no domínio do tempo foram então comparados com os correspondentes resultados obtidos do modelo linear. A Figura 4.28 mostra as respostas no domínio do tempo de algumas das grandezas do sistema para uma súbita aceitação de carga de 15\%, para condição pré-distúrbio nominal.
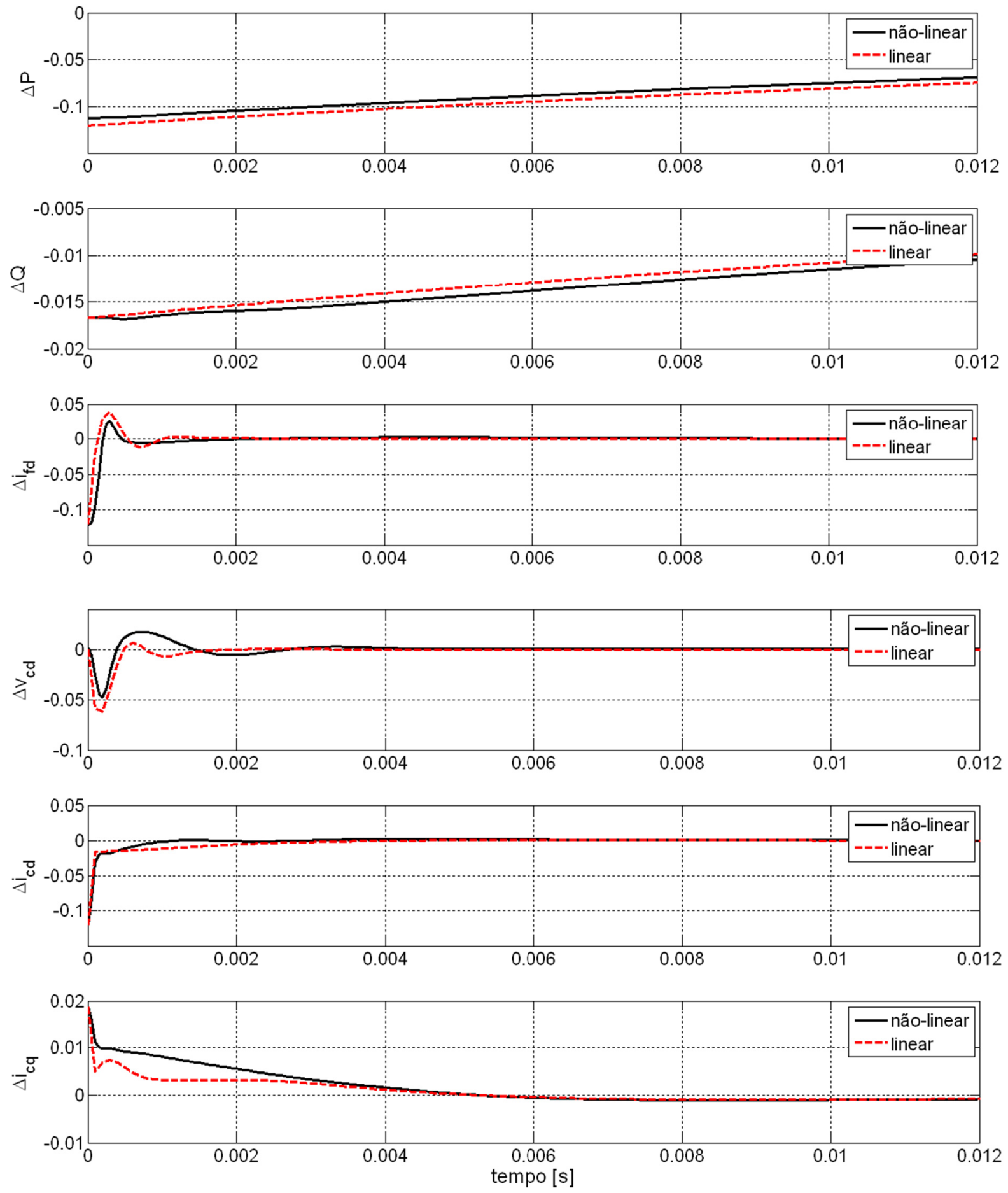

Figura 4.28 - Resposta transitória para $15 \%$ de variação na carga - INV $\mathbf{1}_{\boldsymbol{D}}$ 
A comparação dos resultados obtidos através do modelo não linear com os resultados obtidos do modelo linear indica um padrão semelhante de comportamento das variáveis do sistema, com máximo sobrepasso (overshoot), tempo de assentamento (settling time) e constantes de tempo praticamente similares para os dois casos, até mesmo para valores elevados de distúrbio (15\%). Desta forma, verifica-se qualitativamente a validade do modelo linear nas análises anteriormente realizadas.

Carga Dinâmica - Motor de Indução

Para analisar o comportamento dinâmico do inversor Droop na presença de carga dinâmica, faz-se uso novamente dos motores MI1 e MI2, cujos parâmetros são mostrados na Tabela 4.7. Neste caso, simula-se a conexão do Ml1 e Ml2 diretamente nos terminais do $I N V 1_{D}$ e $I N V 2_{D}$ respectivamente. A Tabela 4.14 mostra os autovalores dos sistemas para operação pré-distúrbio nominal dos motores. O sistema é descrito por catorze variáveis de estado associadas aos inversores e cinco variáveis de estado associadas aos motores, consequentemente, tem-se dezenove autovalores para cada caso. Como pode se observar, com exceção do $\lambda_{18}$ e $\lambda_{19}$, todos os autovalores tem parte real negativa, indicando uma condição de estabilidade para o ponto de operação considerado. $O \lambda_{18}=\lambda_{19}=0$, estão associados à falta de uma referência absoluta para a medição do ângulo $\theta$ e ângulo $\delta$ respectivamente como explicado anteriormente.

A Figura 4.29 mostra os principais autovalores dos sistemas plotados no plano complexo. De forma geral observa-se que a disposição dos autovalores no plano complexo novamente é muito similar para os dois sistemas em estudo. Entretanto, diferentemente do cenário com carga passiva, se observa que a presença do motor gera dois modos oscilatórios pouco amortecidos $(\zeta<0.4)$ de baixa frequência que influenciam negativamente a estabilidade do sistema. O caso mais crítico se registra para o sistema $I N V 1_{D}+M I 1 \operatorname{com} \zeta=0.14$. 
Para uma melhor análise dos resultados, os fatores de participação das variáveis de estado foram calculados. O resultado se mostra na Figura 4.30 onde os fatores de participação com valor inferior a 0.1 foram suprimidos por conveniência. Um gráfico similar pode ser obtido para o sistema $I N V 2_{D}+M I 2$

\begin{tabular}{|c|c|c|}
\hline Autovalor & $I N V 1_{D}+M I 1$ & $I N V 2_{D}+M I 2$ \\
\hline 1,2 & $-1.30 e 6 \pm j 376.47$ & $-2.77 e 6 \pm j 375.4$ \\
\hline 3,4 & $-3728.3 \pm j 8042$ & $-3923.2 \pm j 9055.1$ \\
\hline 5,6 & $-3589.4 \pm j 7554.7$ & $-3834.9 \pm j 8559.1$ \\
\hline 7,8 & $-1107.3 \pm j 1715.5$ & $-1054 \pm j 1616.3$ \\
\hline 9,10 & $-1050.2 \pm j 1627$ & $-994.02 \pm j 1527.5$ \\
\hline 11,12 & $-52.15 \pm j 348.75$ & $-57.83 \pm j 341.15 j$ \\
\hline 13,14 & $-13.59 \pm j 80.12$ & $-12.70 \pm j 66.77$ \\
\hline 15 & -63.15 & -62.81 \\
\hline 16 & -37.35 & -35.94 \\
\hline 17 & -14.81 & -13.23 \\
\hline 18 & 0 & 0 \\
\hline 19 & 0 & 0 \\
\hline
\end{tabular}
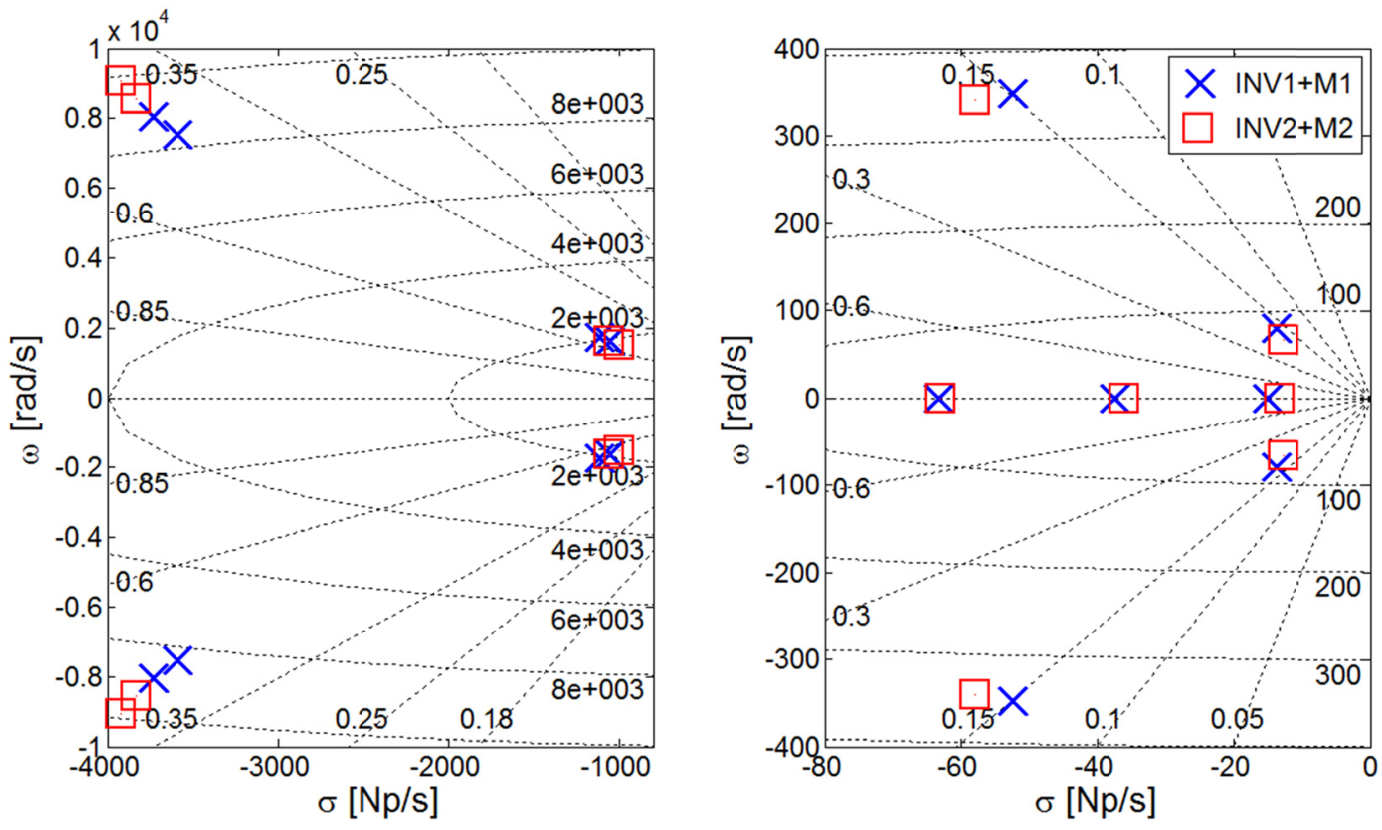

Figura 4.29 - Autovalores do Sistema - INV $V_{D}+M I 1$ 
Como esperado, observa-se que os dois pares de autovalores oscilatórios pouco amortecidos estão associados à dinâmica do motor. O primeiro $\lambda_{11,12}$ está relacionado às correntes transitórias em CC presentes nos enrolamentos do estator, com frequência amortecida próxima aos $60 \mathrm{~Hz}$, enquanto o segundo par $\lambda_{13,14}$ está relacionado com o transitório elétrico presente no rotor. $\mathrm{O} \lambda_{17}$ está relacionado aos enlaces de fluxo do eixo de quadratura do rotor do motor, consequentemente ao escorregamento da máquina como explicado anteriormente.

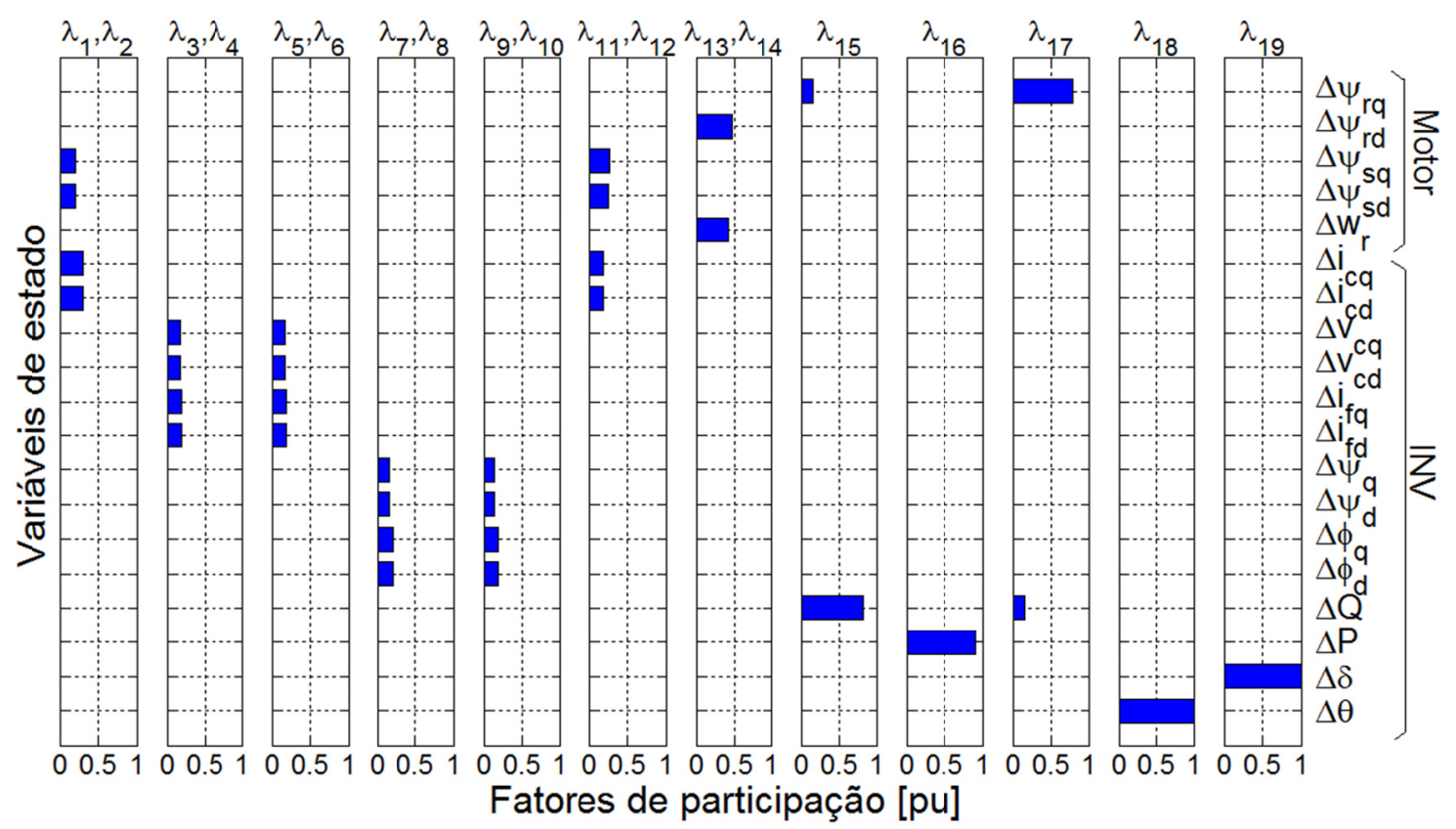

Figura 4.30 - Fatores de participação - INV1 $+M I 1$

É importante observar que os modos oscilatórios relacionados ao inversor, apresentam pouca variação na presença do motor. Se comparado com o sistema GGD+MI (Figura 4.15), o sistema $I N V_{D}+M I$ mostra de forma geral maior margem de estabilidade dinâmica.

Para analisar o impacto da sobrecarga do MI na estabilidade dinâmica do sistema, uma análise de sensibilidade dos autovalores ao torque mecânico aplicado ao motor foi realizada. Para isto foi simulada a conexão do Ml1 diretamente nos terminais do 
$I N V 2_{D}$. A Figura 4.31 mostra o lugar geométrico dos modos dominantes para variações no valor pré-distúrbio do torque entre $100 \%$ e $225 \%$, em passos de $25 \%$.

A análise da Figura 4.31 mostra que o $\lambda_{13,14}$ é positivamente sensível ao incremento do torque, aumentando consideravelmente seu valor de $\zeta$. Todavia, a instabilidade neste caso é dada pelo deslocamento à direita do plano complexo do $\lambda_{17}$, indicando uma condição de stall no motor de indução. Já o $\lambda_{11,12}$ é praticamente insensível às variações no torque.

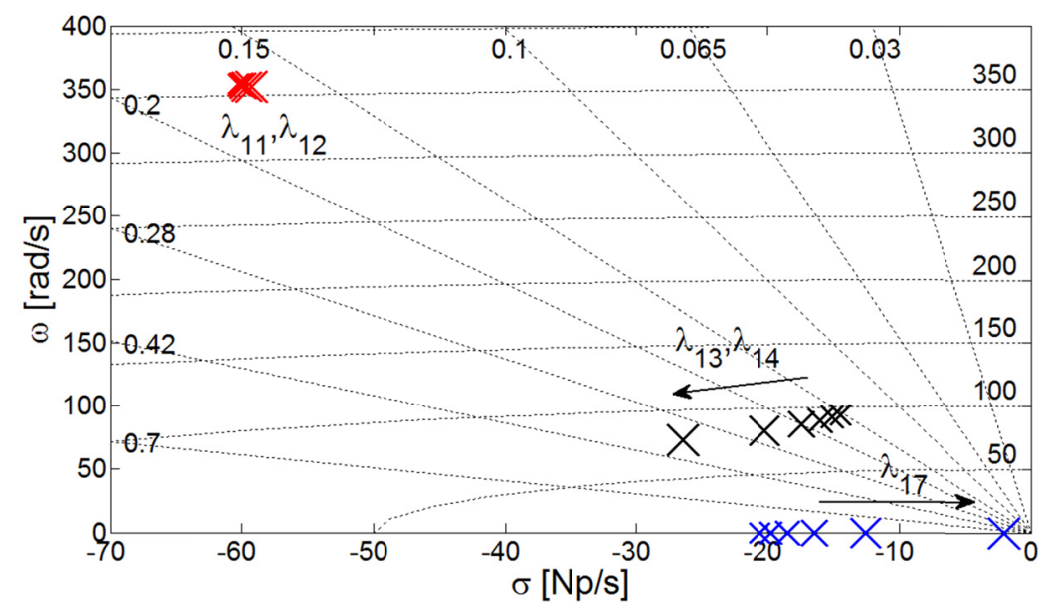

Figura 4.31 - Lugar geométrico do $\lambda_{11,12}, \lambda_{13,14}$ e $\lambda_{17}$ para variações no Torque

Para corroborar os dados obtidos através da análise modal, simulações na plataforma Simulink® foram realizadas com modelos não lineares detalhados do sistema. Os resultados das simulações no domínio do tempo foram então comparados com os correspondentes resultados obtidos do modelo linear. A Figura 4.32 mostra as respostas no domínio do tempo de algumas das grandezas do sistema para um incremento súbito de $15 \%$ no torque mecânico aplicado ao eixo do motor, para condição pré-distúrbio nominal.

A comparação dos resultados obtidos através do modelo não linear com os resultados obtidos do modelo linear indica um padrão semelhante de comportamento das variáveis do sistema, com máximo sobrepasso (overshoot), 
tempo de assentamento (settling time) e constantes de tempo similares para os dois casos, até mesmo para valores elevados de distúrbio (15\%).
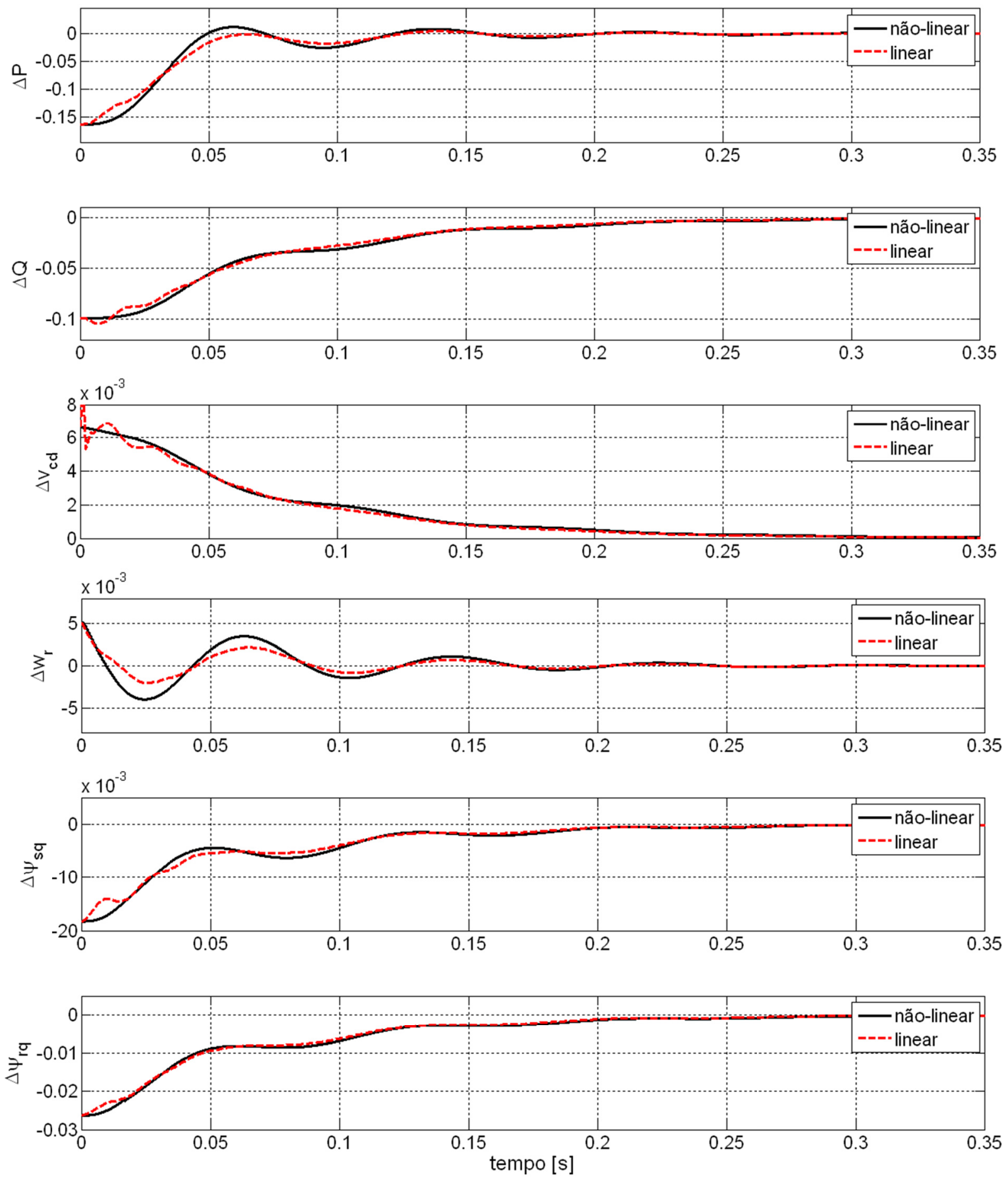

Figura 4.32 - Resposta transitória para $15 \%$ de variação no Torque - INV $\mathbf{1}_{\boldsymbol{D}}+\boldsymbol{M} \mathbf{1}$ 


\subsubsection{Inversor Droop com Restauração de Frequência}

\subsubsection{Ajuste das malhas de controle}

Como mencionado no Capitulo 3, a estratégia de controle deste inversor é similar ao do Controle Droop, com a diferença que este controle apresenta mais uma malha de controle externa para restabelecer a frequência ao seu valor programado. Partindo do mesmo principio utilizado no caso do GGD, um ajuste adequado desta malha deveria garantir sua atuação unicamente depois da atuação do controle primário de frequência. Considerando a rápida resposta do inversor, considera-se apropriado o restabelecimento da frequência ao seu valor programado na faixa de 2 segundos após uma aceitação de carga de $100 \%$. Assim sendo, o ajuste da malha de controle secundário é realizado empiricamente através de simulações no domínio do tempo, tendo como base os inversores com controle Droop analisados na seção anterior. Os parâmetros das malhas de controle secundário, assim ajustados, se mostram na Tabela 4.15.

Tabela 4.15 - Ganhos da malha de controle secundário- Controle Droop+F

\begin{tabular}{ccc}
\hline Parâmetro & $\boldsymbol{I N V 1 _ { D + F }}$ & $\boldsymbol{I N V 2 _ { D + F }}$ \\
\hline $\boldsymbol{K}_{\boldsymbol{p 0 - D} \boldsymbol{D}}[\boldsymbol{p u}]$ & 0.003 & 0.004 \\
$\boldsymbol{K}_{\boldsymbol{i 0 - D} \boldsymbol{D}}[\boldsymbol{p u}]$ & 120 & 100 \\
\hline
\end{tabular}

\subsubsection{Análise da estabilidade dinâmica}

Carga $R-L$

A Tabela 4.16 mostra os autovalores correspondentes à operação pré-distúrbio nominal dos inversores, com carga $R-L, f p=0.8$, diretamente ligado aos seus terminais. O sistema é descrito por quinze variáveis de estado associadas aos modelos no espaço de estados dos Inversores com Controle Droop $+F\left(I N V_{D+F}\right)$ e 
duas variáveis de estado associadas aos modelos no espaço de estados das cargas, consequentemente, tem-se dezessete autovalores $(\lambda)$ para cada caso.

Tabela 4.16 - Autovalores do Sistema $-I N V_{D+F}+$ Carga nominal R-L

\begin{tabular}{|c|c|c|}
\hline Autovalor & $I N V 1_{D+F}$ & $I N V 2_{D+F}$ \\
\hline 1,2 & $-9.12 e 5 \pm j 377.02$ & $-1.88 \mathrm{e} 6 \pm \mathrm{j} 376.97$ \\
\hline 3,4 & $-3707.1 \pm j 7311.3$ & $-3895.6 \pm j 8130.4$ \\
\hline 5,6 & $-3390.7 \pm j 6892.3$ & $-3610.2 \pm j 7709.7$ \\
\hline 7,8 & $-1237.3 \pm j 1762.7$ & $-1194.7 \pm j 1655.3$ \\
\hline 9,10 & $-1165.2 \pm j 1735.9$ & $-1127.5 \pm j 1630.8$ \\
\hline 11,12 & $-424.38 \pm j 361.95$ & $-424.04 \pm j 357.73$ \\
\hline 13 & -44.585 & -44.585 \\
\hline 14 & -39.70 & -39.70 \\
\hline 15 & -1.366 & -1.238 \\
\hline 16 & 0 & 0 \\
\hline 17 & 0 & 0 \\
\hline
\end{tabular}

Se comparado com os autovalores do Controle Droop, a análise da tabela acima mostra que a malha de controle secundário tem o efeito de incluir um modo real negativo no sistema $\left(\lambda_{15}\right)$, sendo que os autovalores restantes praticamente não são afetados por esta malha de controle.

A sensibilidade dos autovalores do $I N V 1_{D+F}$ para variações no parâmetro $K_{i 0-D}$ entre 60 e 240 (em passo de 60), se mostra na Figura 4.33.

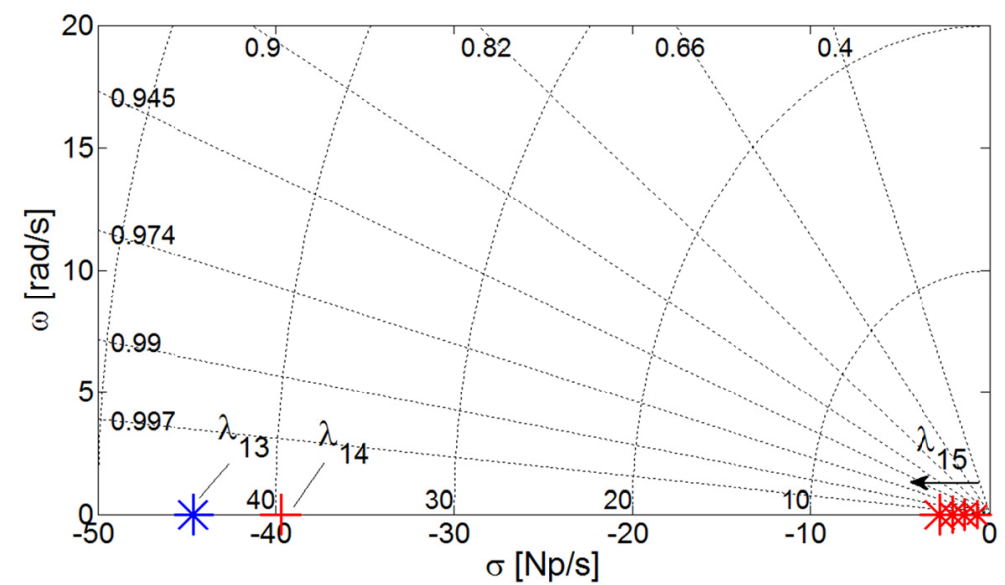

Figura 4.33 - Lugar geométrico do $\lambda_{13}, \lambda_{14}$ e $\lambda_{15}$ para variações de $K_{\mathbf{i} 0-D}$ 
É importante observar que diferentemente do efeito desestabilizante que tem o incremento do ganho da malha de controle secundário no GGD, no caso do inversor verifica-se um deslocamento do $\lambda_{15}$ para a esquerda do plano complexo como função do incremento no valor de $K_{i 0-D}$, enquanto os outros modos dominantes $\left(\lambda_{13} e \lambda_{14}\right)$ são praticamente insensíveis as variações. Resultado similar se obtém para as variações no parâmetro $K_{p 0-D}$.

Carga Dinâmica - Motor de Indução

De forma similar ao caso da carga R-L, o controle secundário de frequência afeta ligeiramente a posição dos autovalores mostrados na Tabela 4.14, referentes ao sistema formado pelo motor de indução diretamente ligado no inversor Droop.

\subsubsection{Inversor $P$ - $Q$}

\subsubsection{Ajuste das malhas de controle}

Inversores com controle $\mathrm{P}-\mathrm{Q}$ disponíveis no mercado (para aplicações em MG) são geralmente projetados para servir de interface aos painéis fotovoltaicos e/ou turbinas eólicas de pequeno porte. As potências destes inversores estão na faixa de unidades a dezenas de kW. Neste trabalho analisaremos dois inversores trifásicos com potências nominais de $10 \mathrm{~kW}$ e $20 \mathrm{~kW}$, com as características elétricas mostradas na Tabela 4.17 [62].

$\mathrm{Na}$ ausência de uma norma técnica que aborde especificamente o tema relacionado à resposta transitória do Inversor, para o ajuste das malhas empregou-se um procedimento similar ao utilizado no Inversor Droop. Neste caso, para o ajuste preliminar das malhas utilizou-se uma largura de banda na ordem de $1.5 \mathrm{kHz}$ para a malha interna de corrente, $200 \mathrm{~Hz}$ para a malha do PLL e $10 \mathrm{~Hz}$ para a malha de potência [48]. Para todas as malhas, a margem de fase foi mantida entre $40^{\circ}$ e $60^{\circ}$. 
Os ajustes finais (através do enfoque dos autovalores) foram realizados simulando a conexão dos inversores num alimentador típico de BT, com potência de curtocircuito de 4 MVA, relação $R / X=1$. $O$ ajuste foi realizado para condições nominais de operação, i.e., injeção de potência ativa nominal com fator de potência unitário.

Tabela 4.17 - Parâmetros dos Inversores - Controle P-Q

\begin{tabular}{ccc}
\hline Parâmetro & $\boldsymbol{I N V}_{\boldsymbol{P Q}}$ & $\boldsymbol{I N V \boldsymbol { 2 } _ { \boldsymbol { P Q } }}$ \\
\hline $\mathrm{P}[\mathrm{kW}] / \mathrm{fp}$ & $10 / 0.8^{18}$ & $20 / 0.8$ \\
Tensão $[\mathrm{V}]$ & $220 / 127$ & $220 / 127$ \\
$\boldsymbol{f}[\boldsymbol{H z}] / \boldsymbol{w}_{\boldsymbol{s w}}[\boldsymbol{k H z}]$ & $60 / 10$ & $60 / 10$ \\
No. Pulsos & 6 & 6 \\
THD corrente & $<3 \%$ & $<3 \%$ \\
\hline
\end{tabular}

Os valores finais dos ganhos das malhas de controle assim como dos parâmetros da interface do inversor, calculada aplicando-se a metodologia mostrada na Seção 4.2.1, se mostram na Tabela 4.18.

Tabela 4.18 - Ganhos das malhas de controle - Controle P-Q

\begin{tabular}{|c|c|c|}
\hline Parâmetro & $I N V 1_{P Q}$ & $I N V 2_{P Q}$ \\
\hline$R_{c} / L_{c}[p u]$ & $0.008 / 0.079$ & $0.008 / 0.08$ \\
\hline$R_{f} / L_{f} / C_{f}[p u]$ & $0.002 / 0.02 / 0.045$ & $0.002 / 0.019 / 0.039$ \\
\hline$K_{p 0-P Q} / K_{i 0-P Q}[p u]$ & $1.905 / 1200.7$ & $2.001 / 1334.1$ \\
\hline$K_{p 1-P Q} / K_{i 1-P Q}[p u]$ & $0.971 / 122.93$ & 0.754 / 108.33 \\
\hline$K_{p 2-P Q} / K_{i 2-P Q}[p u]$ & 0.547 / 849.26 & 0.5702 / 808.02 \\
\hline$F_{c-P Q}$ & 0.62 & 0.6 \\
\hline$w_{c}[\mathrm{rad} / \mathrm{s}]$ & 40 & 40 \\
\hline
\end{tabular}

As simulações realizadas no domínio do tempo mostraram o atendimento aos limites de distorção harmônica mostrados na Tabela 4.9 e Tabela 4.10, para os valores acima indicados.

\footnotetext{
${ }^{18}$ A maioria de inversores comerciais $\mathrm{P}-\mathrm{Q}$ opera em fp unitário. Entretanto, para analisar o impacto da injeção de reativos na estabilidade dinâmica da MG, neste trabalho utiliza-se um fp diferente de 1.0
} 


\subsubsection{Análise da Estabilidade Dinâmica}

A Tabela 4.19 mostra os autovalores correspondentes à operação pré-distúrbio nominal dos inversores quando conectados num alimentador típico de BT, com potência de curto-circuito de $4 \mathrm{MVA}$, relação $X / R=1$. O sistema é descrito por quinze variáveis de estado correspondentes aos modelos no espaço de estados dos Inversores e duas variáveis de estado associadas ao modelo do alimentador de BT, totalizando dezessete autovalores $(\lambda)$ para cada caso. Como pode se observar, com exceção do $\lambda_{17}$ todos os autovalores têm parte real negativa, indicando uma condição de estabilidade para o ponto de operação considerado. O autovalor $\lambda_{17}$ está associado à falta de uma referência absoluta para medição do ângulo $\theta$.

Tabela 4.19 - Autovalores do Sistema - IN $V_{P Q}$

\begin{tabular}{|c|c|c|}
\hline Autovalor & $I N V 1_{P Q}$ & $I N V 2_{P Q}$ \\
\hline 1,2 & $-1.37 e 12 \pm j 376.99$ & $-3.78 e^{2} \pm j 376.99$ \\
\hline 3,4 & $-3674.5 \pm j 8482.4$ & $-4124.9 \pm j 9370.6$ \\
\hline 5,6 & $-2983.1 \pm j 8696.6$ & $-3384.6 \pm j 9542.5$ \\
\hline 7,8 & $-2293.3 \pm j 1744.9$ & $-2390.9 \pm j 1440.9$ \\
\hline 9,10 & $-1359.4 \pm j 1782.0$ & $-1395 \pm j 1609.0$ \\
\hline 11,12 & $-408.7 \pm j 823.41$ & $-446.24 \pm j 837.85$ \\
\hline 13,14 & $-39.461 \pm j 58.239$ & $-35.133 \pm j 55.982$ \\
\hline 15,16 & $-39.972 \pm j 57.81$ & $-35.637 \pm j 55.662$ \\
\hline 17 & 0 & 0 \\
\hline
\end{tabular}

A Figura 4.34 mostra os principais autovalores dos sistemas plotados no plano complexo. De forma geral, observa-se uma disposição similar dos autovalores para os dois inversores analisados, apresentando o $I N V 2_{P Q}$ um maior fator de amortecimento nos modos de alta frequência. Como esperado, os autovalores estão localizados em três regiões de frequência bem definidas, correspondentes às larguras de banda utilizadas no projeto das três malhas de controle. 

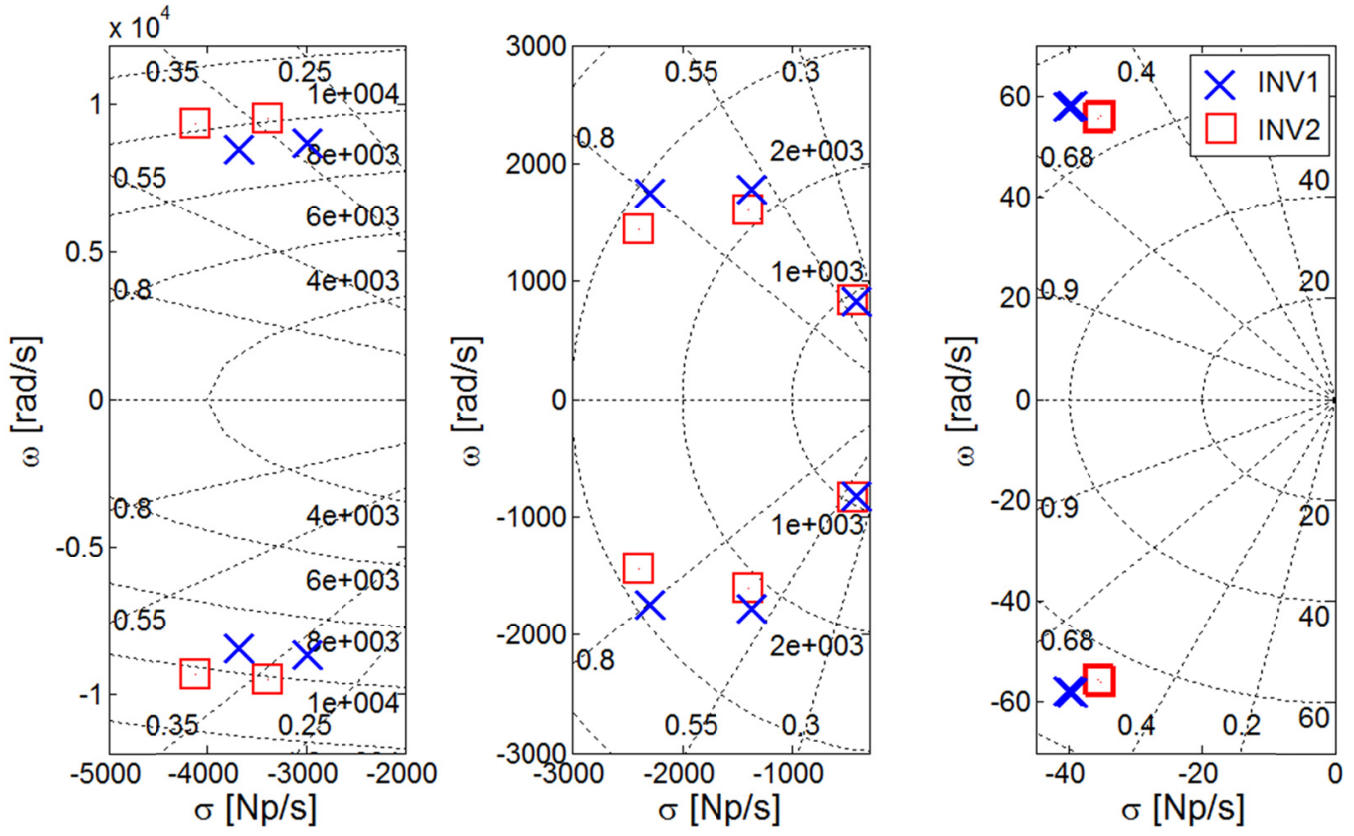

Figura 4.34 - Autovalores do sistema - INV $P Q$

Para uma melhor compreensão do sistema, os fatores de participação foram calculados para o $I N V 1_{P Q}$. O resultado se mostra na Figura 4.35 , onde os fatores de participação com valor inferior a 0.1 foram suprimidos por conveniência.

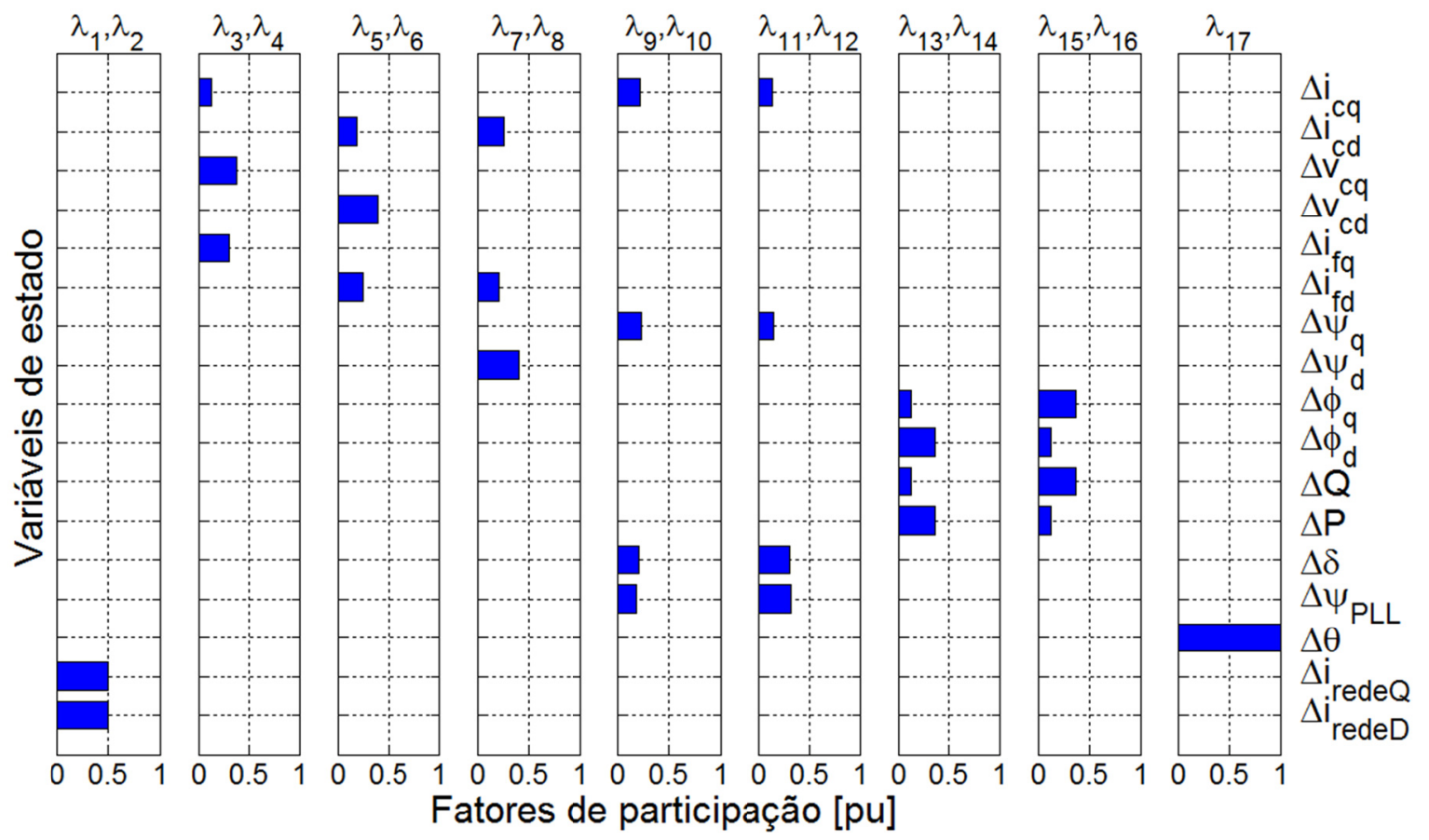

Figura 4.35 - Fatores de participação INV1 $P Q$ 
A análise da Figura 4.35 mostra que o par de autovalores $\lambda_{1,2}$, com frequência amortecida próxima $60 \mathrm{~Hz}$, está relacionado com as correntes transitórias de CC presentes na rede. Os modos $\lambda_{3,4}$ e $\lambda_{5,6}$ estão associados à malha de controle de corrente, com $w_{n}$ próximo a $1.5 \mathrm{kHz}$. A participação das variáveis de estado nos modos $\lambda_{7,8}$, e $\lambda_{9,10}$ se mostra pouco definida, observando-se uma contribuição tanto da malha de corrente como da malha do PLL. Pelo contrário, o $\lambda_{11,12}$ se mostra principalmente relacionado à malha de controle do PLL. Já os modos dominantes $\lambda_{13,14}$ e $\lambda_{15,16}$ estão relacionados ao controle de potência ativa e reativa respectivamente, com $w_{n}$ na faixa de $10 \mathrm{~Hz}$. É importante notar que devido à característica resistiva do alimentador $(R / X=1)$, o controle de potência ativa e reativa neste caso não é totalmente desacoplado, observando-se uma leve participação de $\Delta P$ e $\Delta \emptyset_{d}$ no $\lambda_{15,16}$ e de $\Delta Q$ e $\Delta \emptyset_{q}$ no $\lambda_{13,14}$. Também é importante observar que, diferentemente do controle Droop, neste caso os modos dominantes do sistema são complexos, consequentemente a resposta transitória do sistema apresenta oscilações de baixa frequência. Já o $\lambda_{17}$ está relacionado à falta de uma referência absoluta para medição do ângulo $\theta$ como explicado anteriormente.

A Figura 4.36 e Figura 4.37 mostram o lugar geométrico da parte real $(\sigma)$, imaginária $(w)$, fator de amortecimento $(\zeta)$, e frequência natural $\left(w_{n}\right)$ do par de autovalores dominantes do sistema $\left(\lambda_{13,14}\right.$ e $\left.\lambda_{15,16}\right)$ para variações na potência ativa $(P)$ e reativa (Q) injetada pelo inversor na condição pré-distúrbio. A potência ativa foi variada entre 0 e $1.0 \mathrm{pu}$, e a potência reativa entre -0.75 pu (absorção de reativos) a +0.75 pu (injeção de reativos).

A análise das figuras mostra que $\circ \zeta$ dos modos oscilatórios do sistema é praticamente insensível às variações no $P$ e $Q$ injetados pelo inversor na rede, observando-se o maior valor de $\zeta$ para injeção de $P$ nominal. Já a variação no $Q$, produz uma migração oposta no $\zeta$ dos modos, tendo o $\lambda_{13,14}$ um leve aumento de $\zeta$ para $Q$ positivos, e o $\lambda_{15,16}$ para $Q$ negativos. A frequência dos modos também apresenta uma variação pequena, observando-se um aumento de $w_{n}$ para $Q$ positivos, com praticamente nenhuma sensibilidade às variações no $P$. 

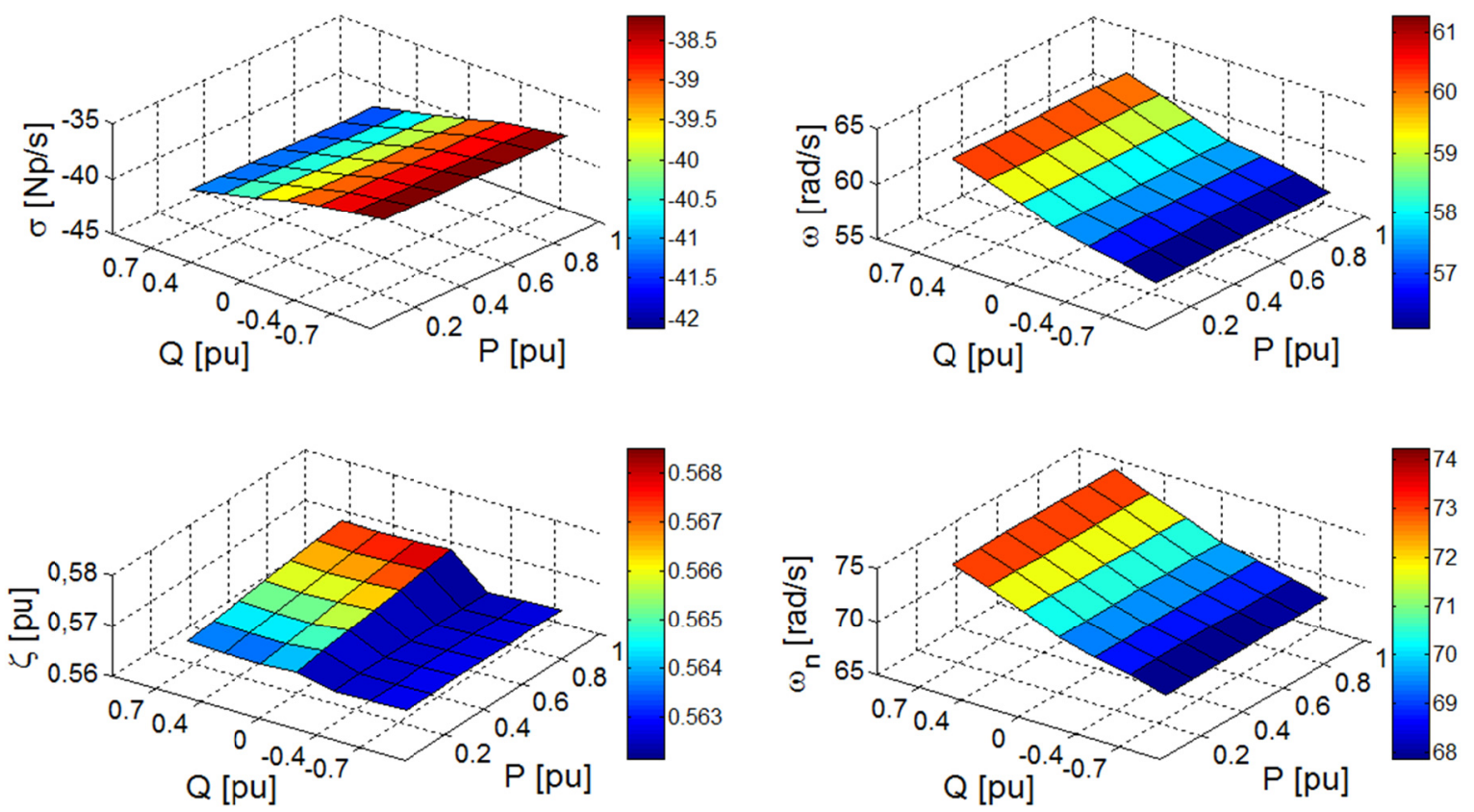

Figura 4.36 - Lugar geométrico do $\lambda_{13,14}$ para variações de $\mathrm{P}$ e $\mathrm{Q}$ - INV1 $1_{P Q}$
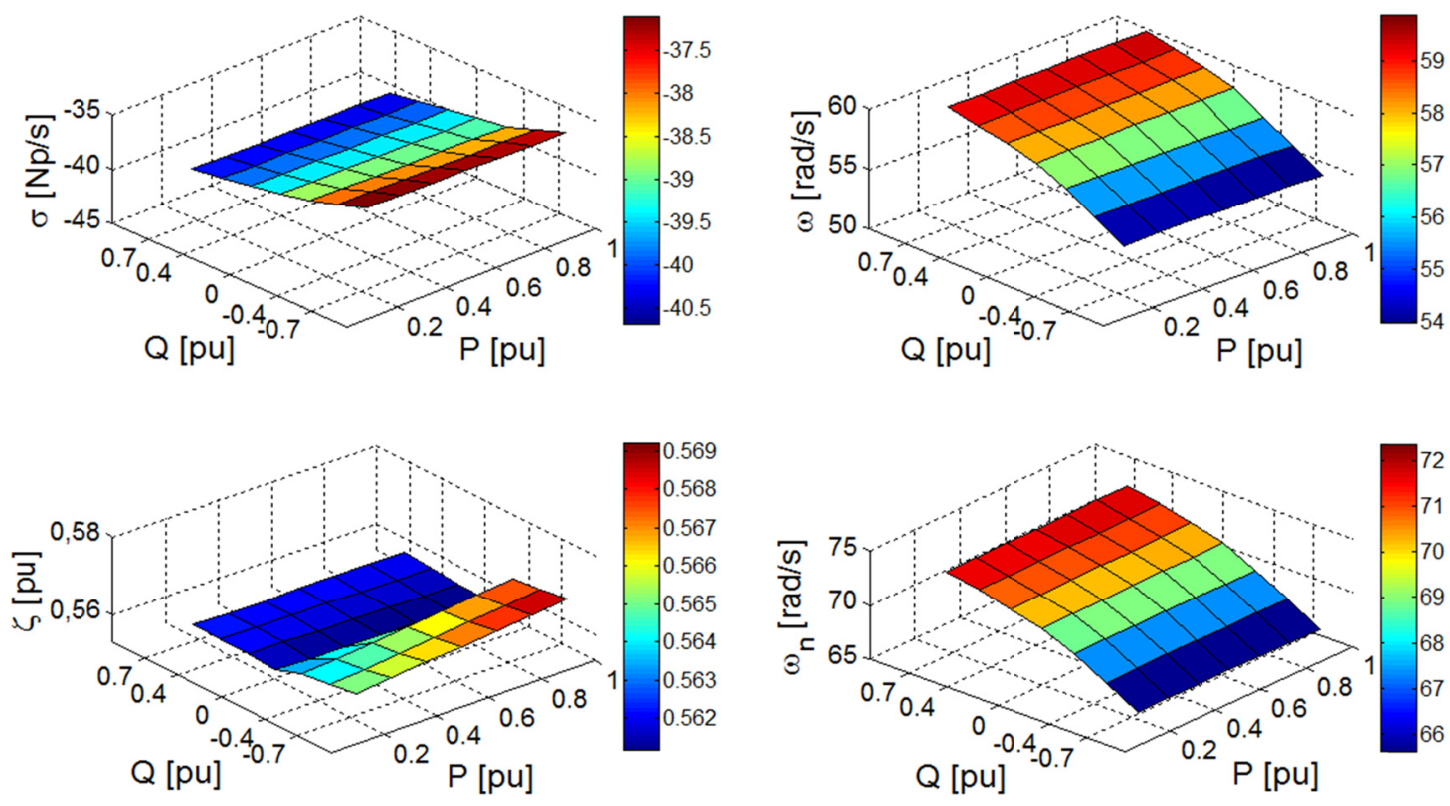

Figura 4.37 - Lugar geométrico do $\lambda_{15,16}$ para variações de $\mathrm{P}$ e $\mathrm{Q}-\boldsymbol{I N V} \mathbf{1}_{P Q}$

Para validar os resultados obtidos através da análise modal, simulações na plataforma Simulink® foram realizadas com modelos não lineares detalhados do sistema. Os resultados das simulações no domínio do tempo foram então comparados com os correspondentes resultados obtidos do modelo linear. A Figura 
4.38 mostra as respostas no domínio do tempo de algumas das grandezas do sistema para uma variação súbita de $15 \%$ no $\mathrm{P}$ injetado pelo inversor, para condição pré-distúrbio nominal.
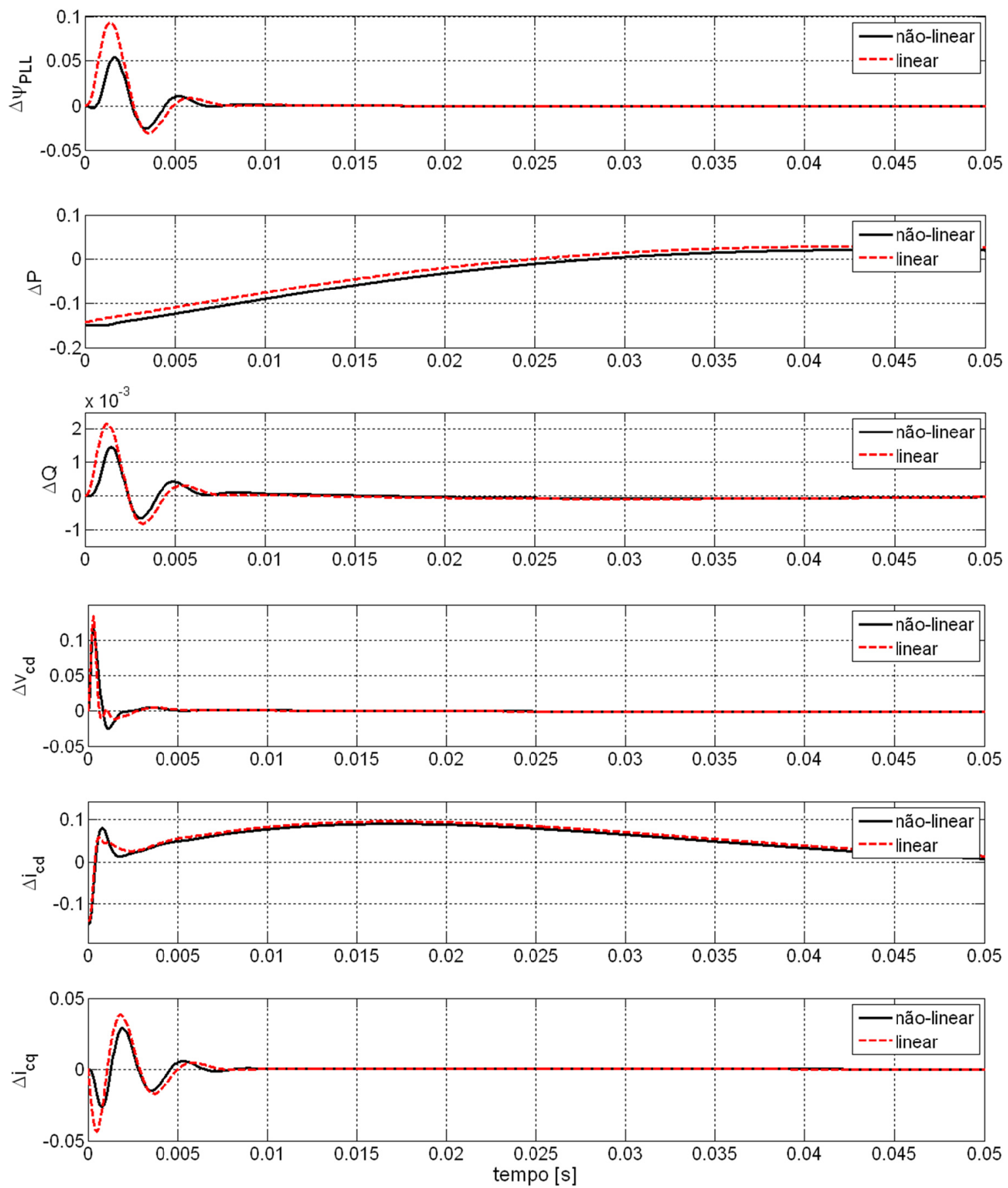

Figura 4.38 - Resposta transitória para $15 \%$ de variação no $P$ do inversor - INV $1_{P Q}$ 
A comparação dos resultados obtidos através do modelo não linear com os resultados obtidos do modelo linear indica um padrão semelhante de comportamento das variáveis do sistema, com máximo sobrepasso (overshoot), tempo de assentamento (settling time) e constantes de tempo praticamente similares para os dois casos, até mesmo para valores elevados de distúrbio (15\%). Desta forma, verifica-se qualitativamente a validade do modelo linear nas análises anteriormente realizadas. 


\section{Análise da Operação Conjunta das FEDs na MG}

No Capítulo 4 foi apresentada e discutida a estabilidade dinâmica das FEDs para operação individual. O principal objetivo deste capítulo é avaliar a estabilidade dinâmica de uma MG ilhada, formada por vários agrupamentos de FEDs, conectadas em paralelo através de alimentadores de BT $(220 \mathrm{~V})$, para o atendimento de cargas residenciais e industriais.

Assim como nos sistemas elétricos convencionais, a configuração de uma MG é um fator que influencia diretamente a maneira como esta responde às pequenas $e$ grandes perturbações do sistema. Devido à grande quantidade de componentes que podem compor uma MG, existe um vasto leque de possibilidades de configurações de Minirredes, fato que a primeira vista dificultaria a análise da estabilidade dinâmica de forma geral.

Porém, fazer uma análise para cada tipo de configuração de MG é uma tarefa desnecessária, pois, partindo de uma única configuração é possível inferir o comportamento dinâmico de um grupo de configurações semelhantes, através de uma análise de sensibilidade dos autovalores às variações nos parâmetros elétricos do sistema.

\subsection{Configuração da Minirrede de teste}

O diagrama unifilar da configuração de MG utilizada para este estudo foi adaptada de [47] e se mostra na Figura 5.1. A MG opera em $220 \mathrm{~V}-60 \mathrm{~Hz}$ e é composta por nove barras (identificados na figura por números) e por oito alimentadores trifásicos (identificados na figura por letras). Um transformador de distribuição MT/BT permite a conexão da MG com a rede de distribuição convencional quando necessário, contudo, para este estudo unicamente se considera a operação ilhada da MG. Dentro da MG existe uma área residencial formada por edifícios de apartamentos e grupos de residências individuais, totalizando uma potência coincidente de $93.5 \mathrm{~kW}$ de carga passiva - fator de potência 0.92 indutivo -, e $15 \mathrm{~kW}$ de carga ativa (motor de indução). Um alimentador expresso alimenta uma pequena indústria com 
potência coincidente de $80 \mathrm{~kW}$ de carga passiva - fator de potência 0.92 indutivo - e $30 \mathrm{~kW}$ de carga ativa. Dentro da área residencial existem quatro FEDs despacháveis formadoras de rede: dois GGDs de $45 \mathrm{~kW}$ de potência nominal cada e duas microturbinas de $30 \mathrm{~kW}$ e $15 \mathrm{~kW}$ de potência nominal, acopladas a rede através de inversores com controle Droop e/ou Droop+F. Na área residencial também existem mais duas FEDs não despacháveis: uma turbina eólica de $10 \mathrm{~kW}$ de potência nominal e um sistema fotovoltaico de $20 \mathrm{~kW}$ de potência nominal; ambas as duas acopladas à rede através de inversores com controle $\mathrm{P}-\mathrm{Q}$. Já na área industrial existe mais uma FED despachável formadora de rede: um GGD de $90 \mathrm{~kW}$ de potência nominal. Os parâmetros elétricos dos alimentadores assim como a distribuição das cargas em cada uma das barras se mostram no Apêndice A.

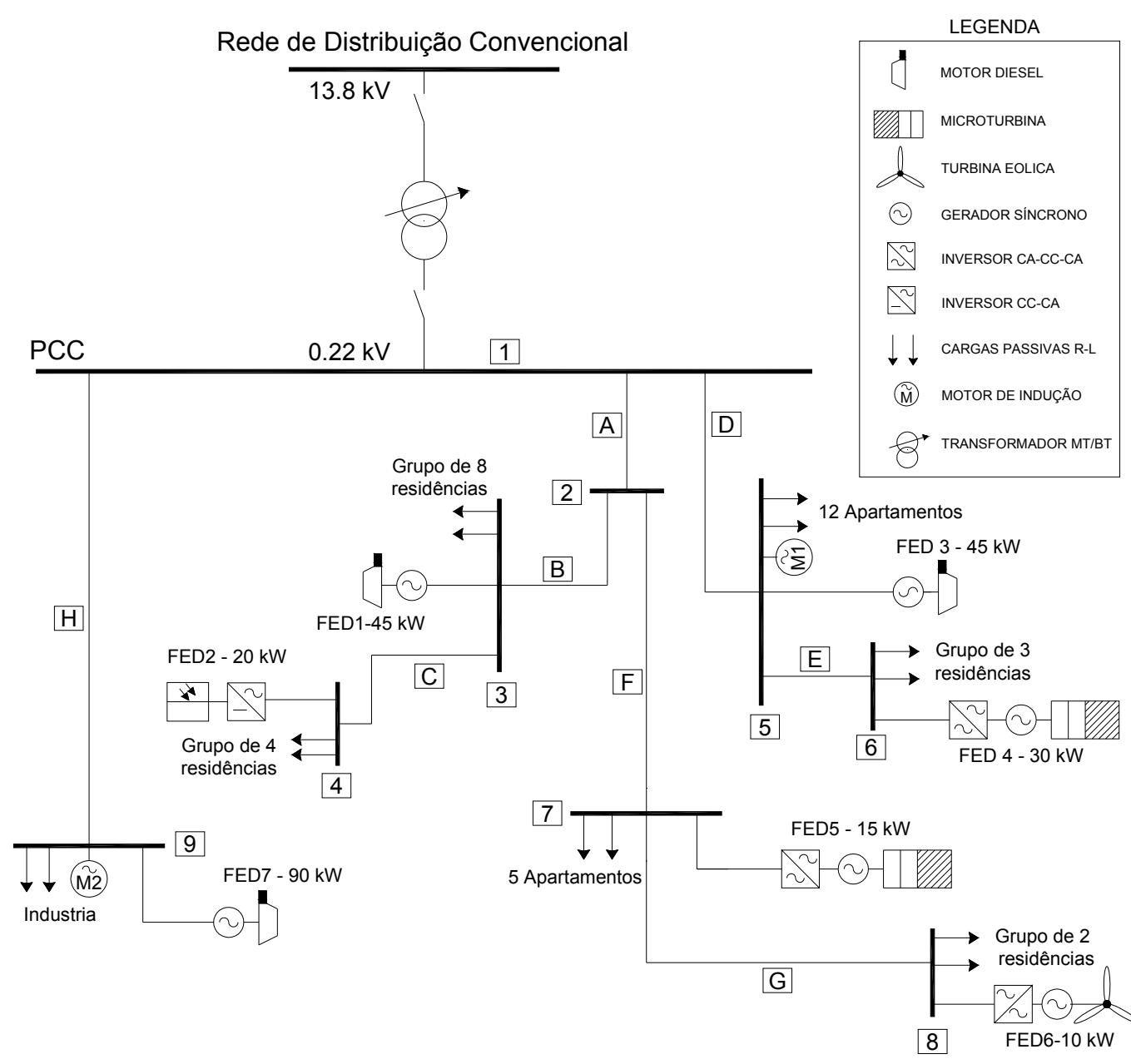

Figura 5.1 - Diagrama unifilar da configuração de MG de teste 
Para um melhor entendimento das interações entre as dinâmicas das diferentes FEDs, assim como da influência dos parâmetros elétricos da rede na estabilidade do sistema, a MG será analisada por partes (casos). Cada caso analisa a estabilidade dinâmica de determinadas áreas da MG, sendo que no final, a estabilidade dinâmica do sistema todo será estudada.

\section{2 Área A}

A Figura 5.2 mostra o diagrama unifilar da primeira área sob estudo. Para facilitar a análise, define-se a operação nominal deste sistema como aquela condição que apresenta carga coincidente máxima (30 kW), com $\mathrm{fp}=0.92$ indutivo, estando o sistema fotovoltaico injetando potência ativa nominal $\left(P_{F E D 2}=1\right)$ com $\mathrm{fp}=1.0$ $\left(Q_{F E D 2}=0\right)$ e o GGD regulando a tensão na barra $3\left(V_{3}\right)$ em 1.0 pu..

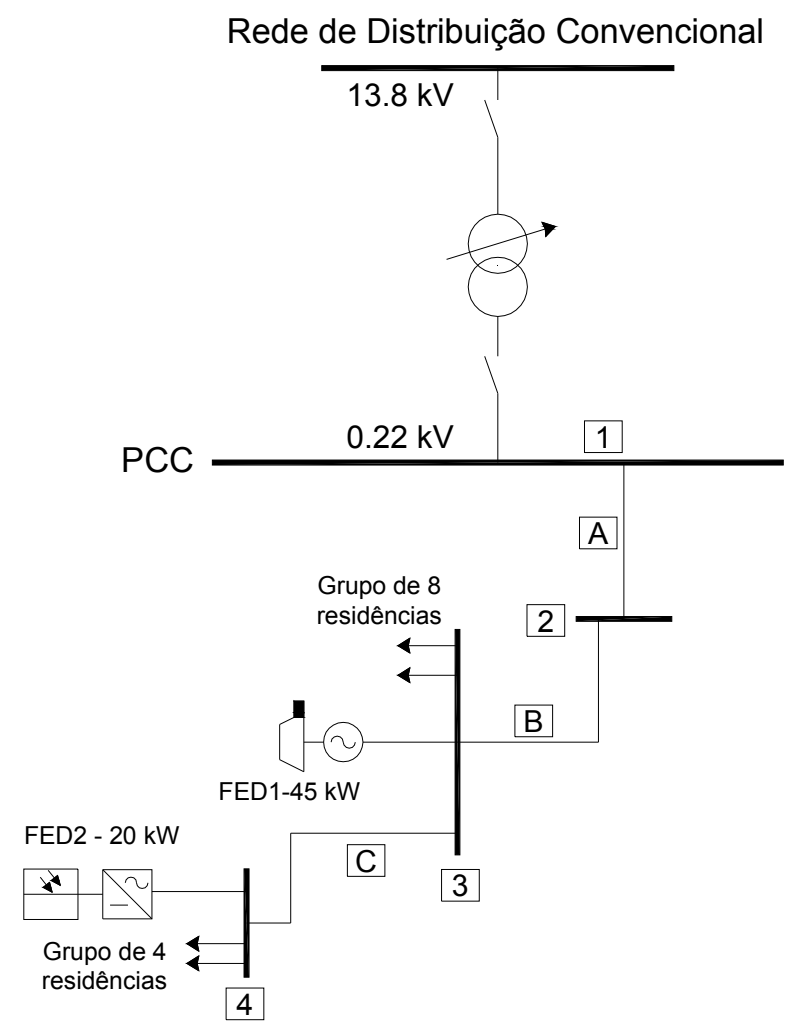

Figura 5.2 - Diagrama Unifilar da MG - Área A 
A Tabela 5.1 mostra os principais autovalores do sistema correspondentes à operação nominal do sistema. Todos os autovalores têm parte real negativa, indicando uma condição de estabilidade para o ponto de operação considerado.

Tabela 5.1 - Autovalores da MG para operação nominal - Área A

\begin{tabular}{cccc}
\hline Autovalor & $\sigma \pm j w$ & Autovalor & $\sigma \pm j w$ \\
\hline 1,2 & $-5081.4 \pm j 9362.8$ & 17,18 & $-35.33 \pm j 56.32$ \\
3,4 & $-4368.3 \pm j 8497.9$ & 19 & -59.493 \\
5,6 & $-1495.5 \pm j 1608.5$ & 20 & -33.142 \\
7,8 & $-385.86 \pm j 1267.7$ & 21 & -15.514 \\
9,10 & $-884.56 \pm j 377.09$ & 22,23 & $-2.142 \pm j 13.81$ \\
11,12 & $-595.16 \pm j 738.07$ & 24,25 & $-2.27 \pm j 10.41$ \\
13,14 & $-737.37 \pm j 378.08$ & 26 & -6.644 \\
15,16 & $-37.16 \pm j 56.99$ & 27 & -0.415 \\
\hline
\end{tabular}

A Figura 5.3 mostra os principais autovalores do sistema plotados no plano complexo.
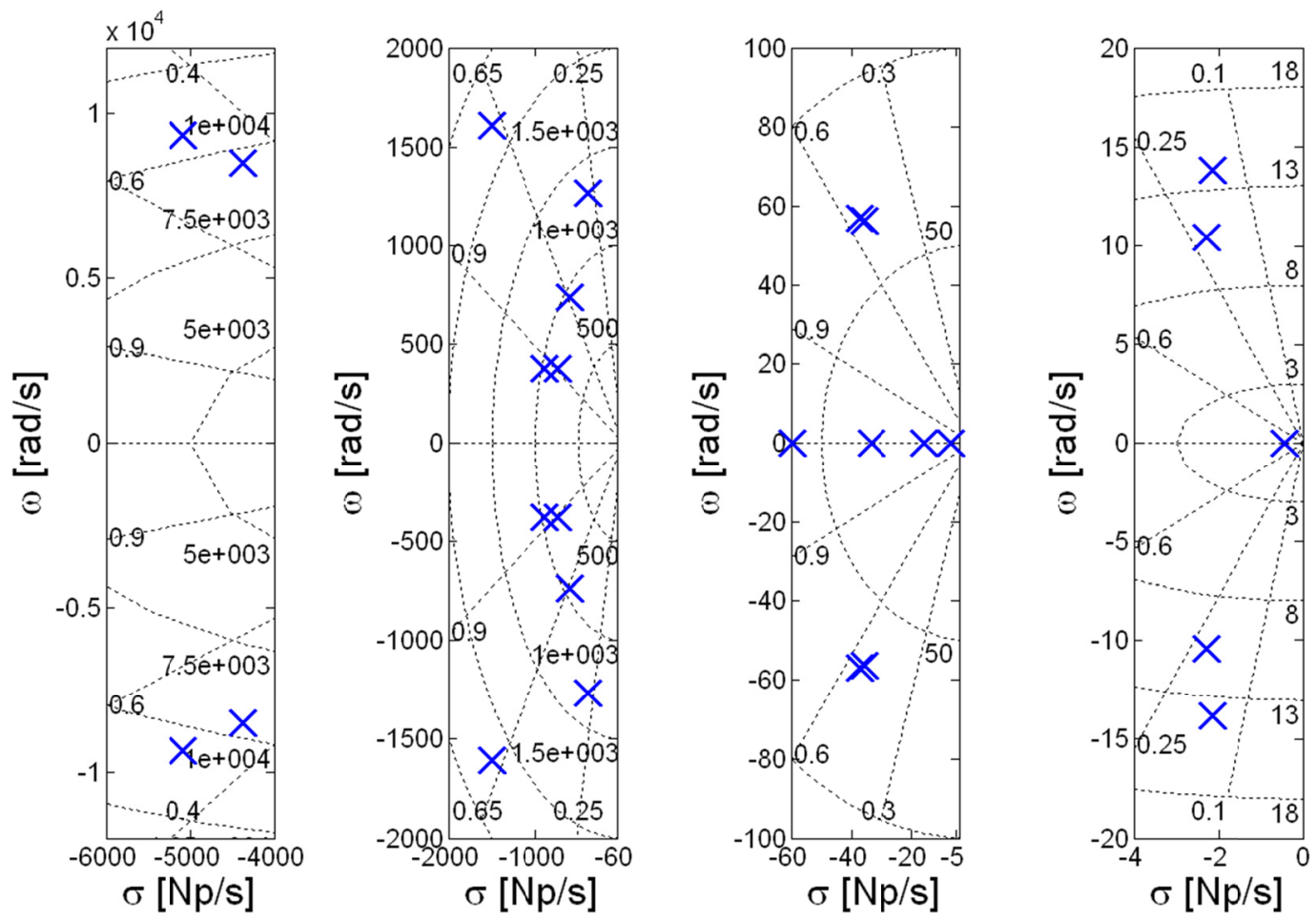

Figura 5.3 - Autovalores da MG para operação nominal - Área A 
A análise da Figura 5.3 mostra um adequado amortecimento de todos os modos, indicando uma boa margem de estabilidade do sistema. Os autovalores que apresentam o menor $\zeta$ são: o modo eletromecânico $\lambda_{22,23}$ associado à dinâmica do motor diesel e do regulador de velocidade do GGD, com $\zeta=0.15$; e o modo eletromecânico $\lambda_{24,25}$ associado ao sistema de excitação do GGD, com $\zeta=0.21$. Todavia, ao comparar estes resultados com os obtidos para operação individual do GGD, observa-se que a presença do sistema fotovoltaico, acoplado à rede através do inversor $P-Q$, tem uma leve influência positiva no $\lambda_{22,23}$, incrementando o $\zeta$ para operações do GGD em regime de carga média e leve. Já os modos associados ao controle de potência ativa e reativa da $F E D_{2}\left(\lambda_{15,16}\right.$ e $\left.\lambda_{17,18}\right)$ não são afetados pela presença do GGD, mostrando valores similares aos obtidos para operação individual da FED. Por outra parte, nota-se uma maior dificuldade por parte da malha do PLL do inversor $P-Q$ em rastrear o ângulo de fase da tensão quando conectado em paralelo com o GGD, observando-se uma diminuição do $\zeta$ do $\lambda_{7,8}$, de 0.44 (para conexão direta na rede convencional) para 0.3 (conectado ao GGD).

Para analisar a influência do $P_{F E D 2}$ e $Q_{F E D 2}$ injetados no sistema, uma análise de sensibilidade foi realizada. Os lugares geométricos dos modos que maiores variações apresentaram se mostra nas figuras a seguir.
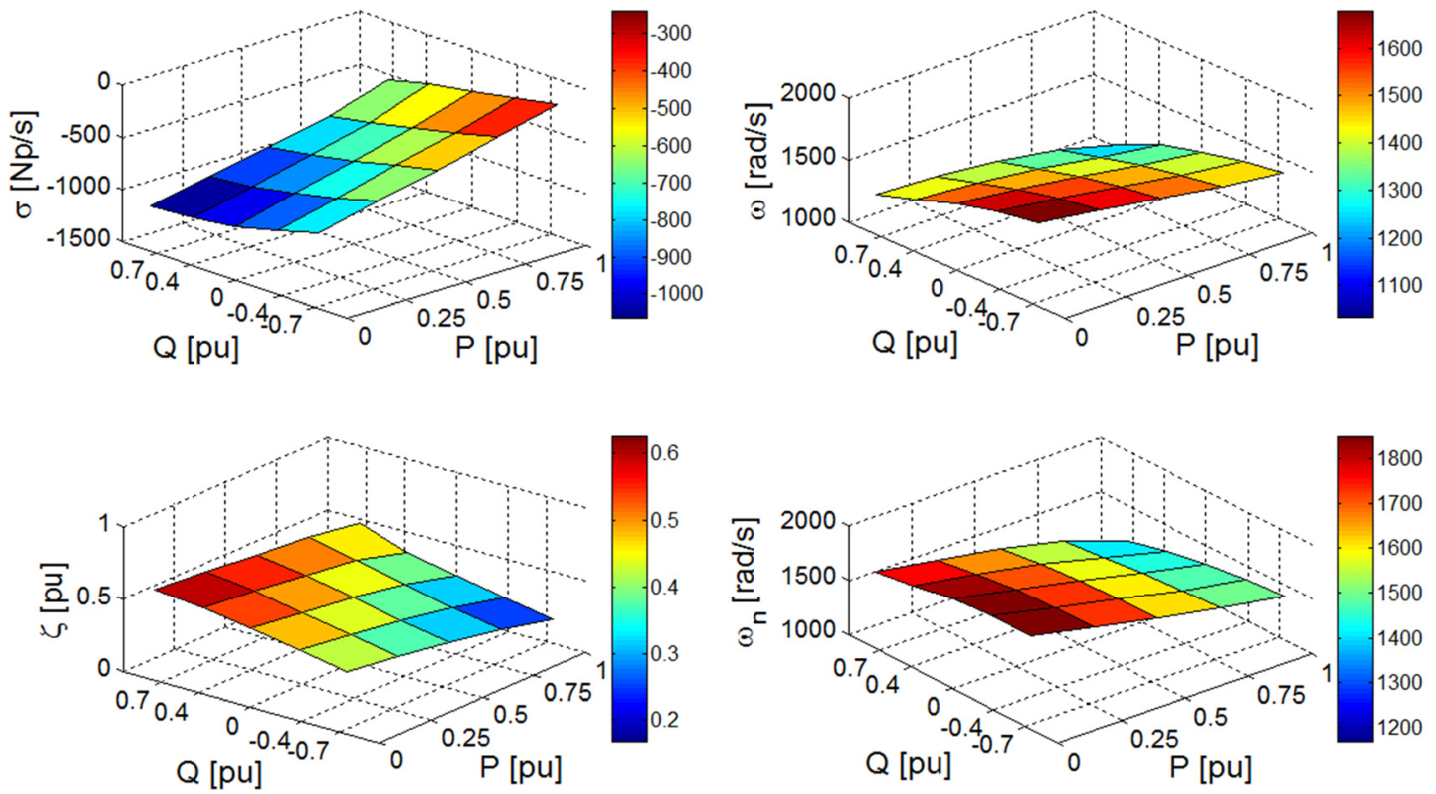

Figura 5.4 - Lugar geométrico do $\lambda_{7,8}$ para variações no $\boldsymbol{P}_{F E D 2}$ e $\boldsymbol{Q}_{F E D 2}-$ Área A 

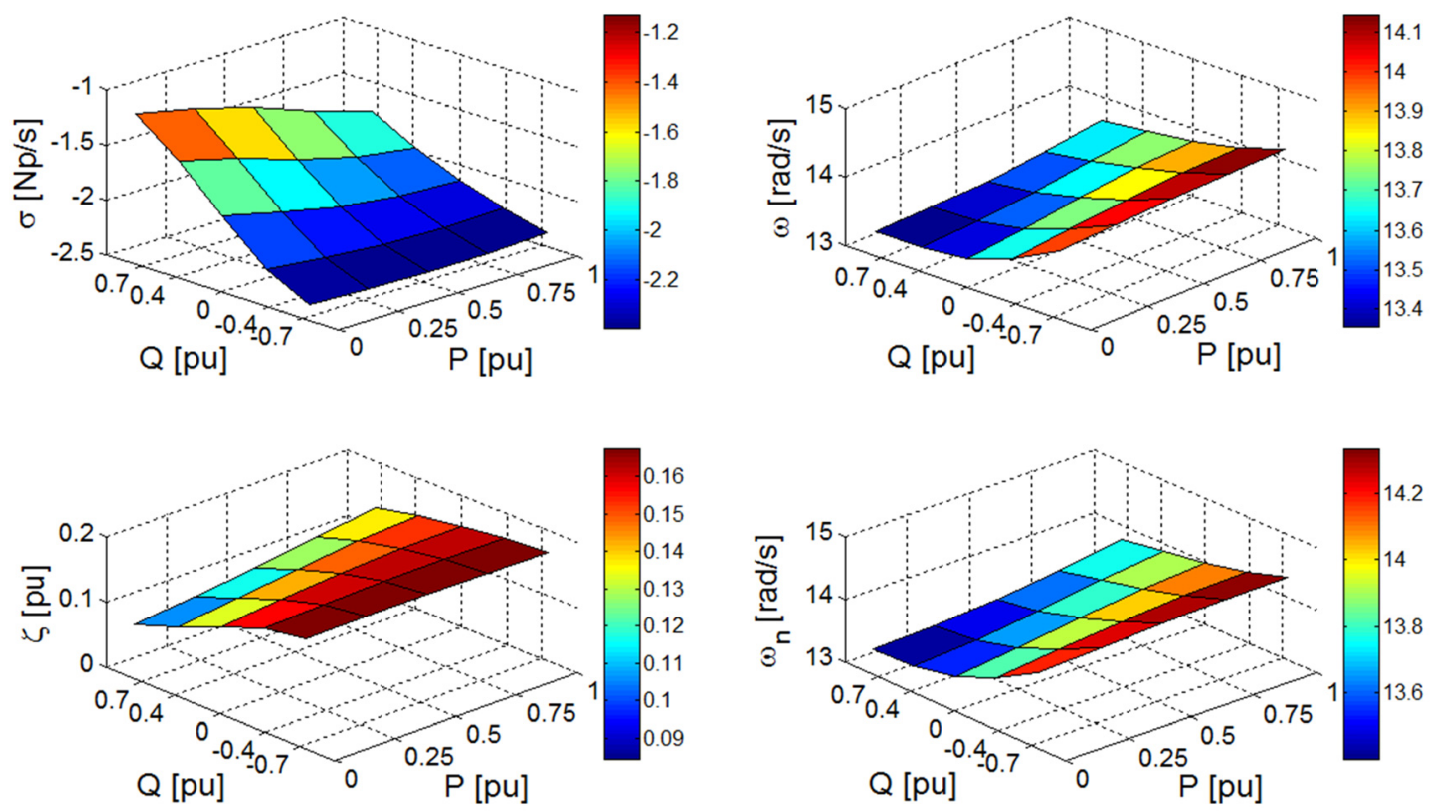

Figura 5.5 - Lugar geométrico do $\lambda_{22,23}$ para variações no $\boldsymbol{P}_{F E D 2}$ e $\boldsymbol{Q}_{F E D 2}$ - Área A
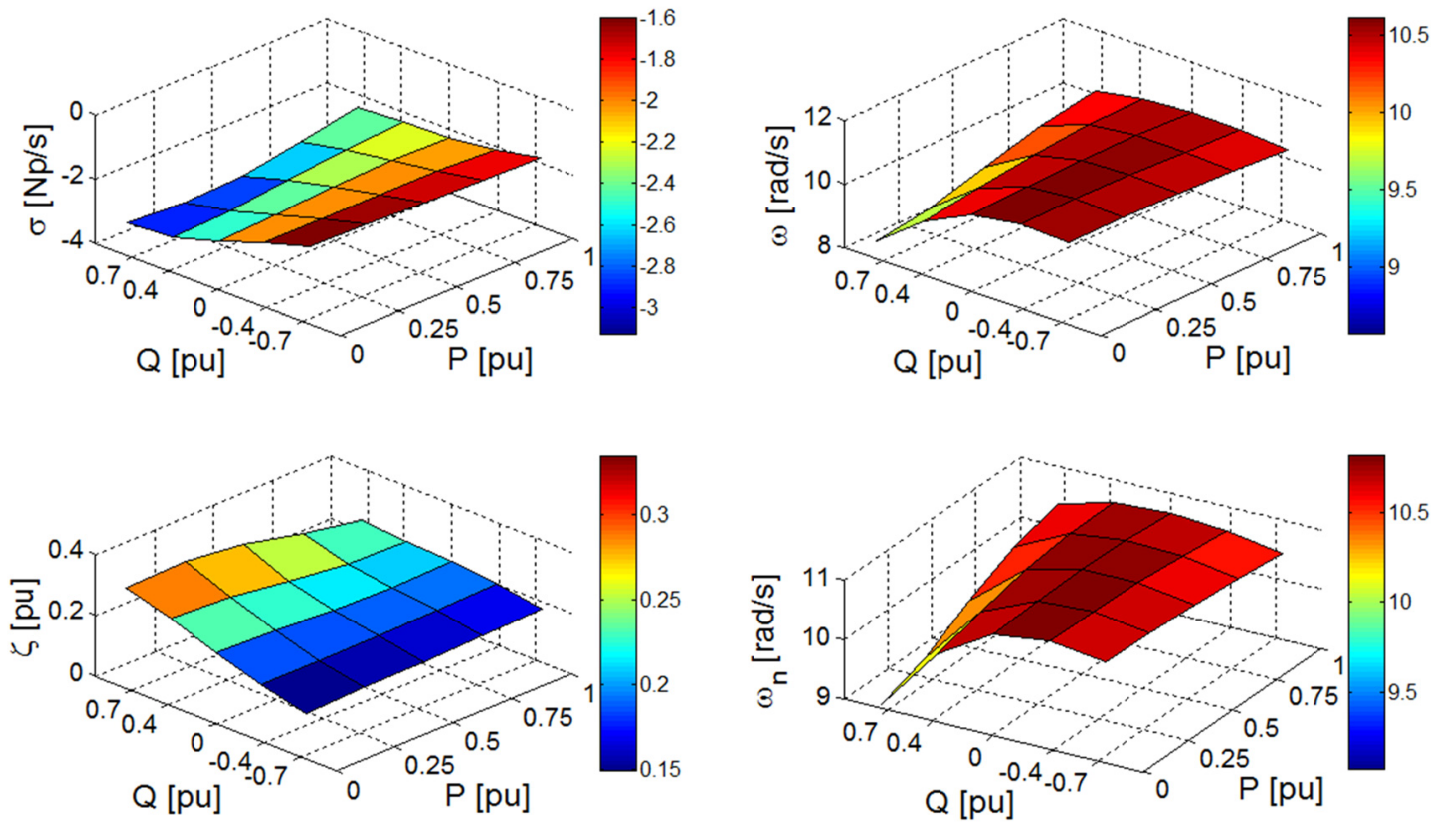

Figura 5.6 - Lugar geométrico $\lambda_{24,25}$ para variações no $\boldsymbol{P}_{F E D 2}$ e $\boldsymbol{Q}_{F E D 2}$ - Área A

A análise das figuras acima mostra que o modo $\lambda_{7,8}$ é muito sensível ao ponto de operação da $F E D_{2}$, observando-se uma sensibilidade negativa do $\zeta$ ao incremento de $P_{F E D 2}$ assim como ao incremento do $Q_{F E D 2}$ absorvido do sistema (Q negativo). $O$ pior caso se registra para a condição pré-distúrbio: $P_{F E D 2}=1$ e $Q_{F E D 2}=-0.75 \mathrm{com}$ 
$\zeta=0.17$. Pelo contrário, o modo eletromecânico $\lambda_{22,23}$ mostra um comportamento oposto, com pouca sensibilidade negativa à diminuição de $P_{F E D 2}$ e uma grande sensibilidade negativa ao incremento de $Q_{F E D 2}$ injetado no sistema ( $Q$ positivo), especialmente para valores baixos de $P_{F E D 2}$, sendo o caso mais crítico a condição pré-distúrbio: $P_{F E D 2}=0$ e $Q_{F E D 2}=+0.75 \mathrm{com} \zeta=0.08$. É importante observar que, embora a injeção de potência reativa por parte da $F E D_{2}$ implique que o fp do GGD aumente, o que a primeira vista deveria incrementar $\circ \zeta$ do seu modo eletromecânico associado ao controle de velocidade (como discutido no Capítulo 4), neste caso se registra um comportamento oposto. Aplicando um raciocínio similar ao caso da potência ativa, também se observa um comportamento contrário, pois à medida que o $P_{F E D 2}$ aumenta, a potência de saída do GGD se reduz, o que deveria diminuir o $\zeta$ deste modo.

Já o modo $\lambda_{24,25}$ é praticamente insensível às variações no $P_{F E D 2}$, e mostra pouca sensibilidade positiva ao incremento de $Q_{F E D 2}$ injetado no sistema, especialmente para valores baixos de $P_{F E D 2}$. Para este modo o pior caso corresponde à condição pré-distúrbio: $P_{F E D 2}=0$ e $Q_{F E D 2}=-0.75 \mathrm{com} \zeta=0.15$. Nota-se que para os dois modos eletromecânicos $\left(\lambda_{22,23}\right.$ e $\left.\lambda_{24,25}\right)$, a frequência de oscilação é praticamente constante é independente da condição pré-distúrbio.

Este é um resultado importante do ponto de vista de projeto de uma MG, pois mostra que a injeção de potência ativa, vinda de uma FED conectada à rede através de um inversor com controle $\mathrm{P}-\mathrm{Q}$, tem um impacto levemente positivo nos modos eletromecânicos do GGD, especialmente no modo associado à dinâmica do motor diesel e do regulador de velocidade. Todavia, este resultado também mostra que a injeção de reativos afeta negativamente a estabilidade dinâmica do GGD. Embora o $\lambda_{7,8}$ apresente uma sensibilidade negativa ao incremento de $P_{F E D 2}$ e do $Q_{F E D_{2}}$ absorvido do sistema, sua frequência de oscilação é elevada, não tendo um impacto pronunciado na resposta transitória do sistema.

Para validar estes resultados foi realizada uma simulação no domínio do tempo na plataforma Simulink $®$, com modelos não lineares detalhados do sistema para três condições pré-distúrbio diferentes. Nos três casos foi simulada uma aceitação de 
carga de $25 \%$ na barra 3, sendo no Caso1 a condição pré-distúrbio: $P_{F E D 2}=0.75 \mathrm{e}$ $Q_{F E D 2}=+0.75$, no Caso 2: $P_{F E D 2}=0.75$ e $Q_{F E D 2}=-0.75$ e no Caso 3: $P_{F E D 2}=0.1 \mathrm{e}$ $Q_{F E D 2}=+0.75$. As oscilações na velocidade do rotor da $F E D_{1}$ se mostram na Figura 5.7 .

Como esperado, se observa que as oscilações eletromecânicas são praticamente insensíveis ao valor pré-distúrbio $P_{F E D 2}$, mostrando o Caso 1 e o Caso 3 um comportamento muito similar. Pelo contrário, nota-se que as variações no $Q_{F E D 2}$ afetam fortemente o amortecimento das oscilações eletromecânicas. Para o Caso 1 e Caso 3, devido ao baixo $\zeta$ do $\lambda_{22,23}$, este modo governa a resposta transitória do sistema, observando-se oscilações menos amortecidas que para o Caso 1. Nota-se também que a frequência de oscilação é similar nos três casos, estando na faixa de $2.0 \mathrm{~Hz}$.

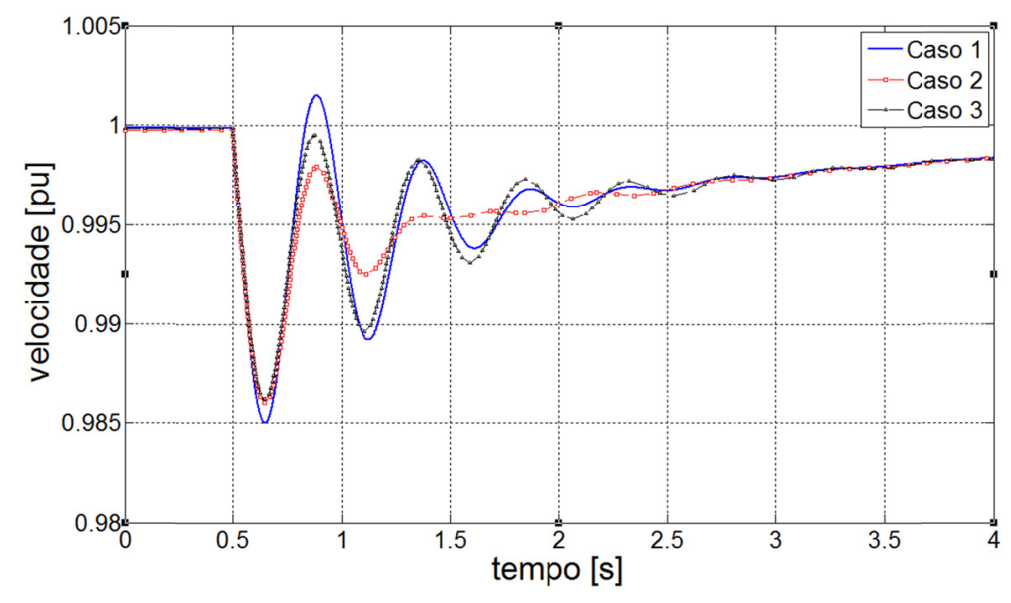

Figura 5.7 - Resposta transitória de velocidade - $\boldsymbol{F} E \boldsymbol{D}_{1}$

Outro estudo de sensibilidade importante de se realizar do ponto de vista de configuração da MG está relacionado aos parâmetros elétricos associados à rede de distribuição, especificamente à impedância dos alimentadores. Neste caso se analisa a sensibilidade dos autovalores às variações no comprimento dos cabos, assim como às variações na relação $R / X$ dos mesmos.

A Figura 5.8 mostra o lugar geométrico dos modos oscilatórios dominantes do sistema em função da distância elétrica entre as FEDs (i.e., em função do comprimento do alimentador $\boldsymbol{C}$ ), para operação nominal do sistema. A distância foi 
variada entre $50 \mathrm{~m}$ e $200 \mathrm{~m}$, em passos de $50 \mathrm{~m}$, mantendo constante a bitola do cabo do alimentador $\boldsymbol{C}$. Por outra parte, a Figura 5.9 mostra o lugar geométrico dos modos oscilatórios dominantes do sistema em função da relação $\mathrm{R} / \mathrm{X}$ do alimentador C. A relação foi variada entre 0.5 e 2.5 , em passos de 0.5 , mantendo constante a reatância do alimentador e alterando unicamente sua resistência ${ }^{19}$.
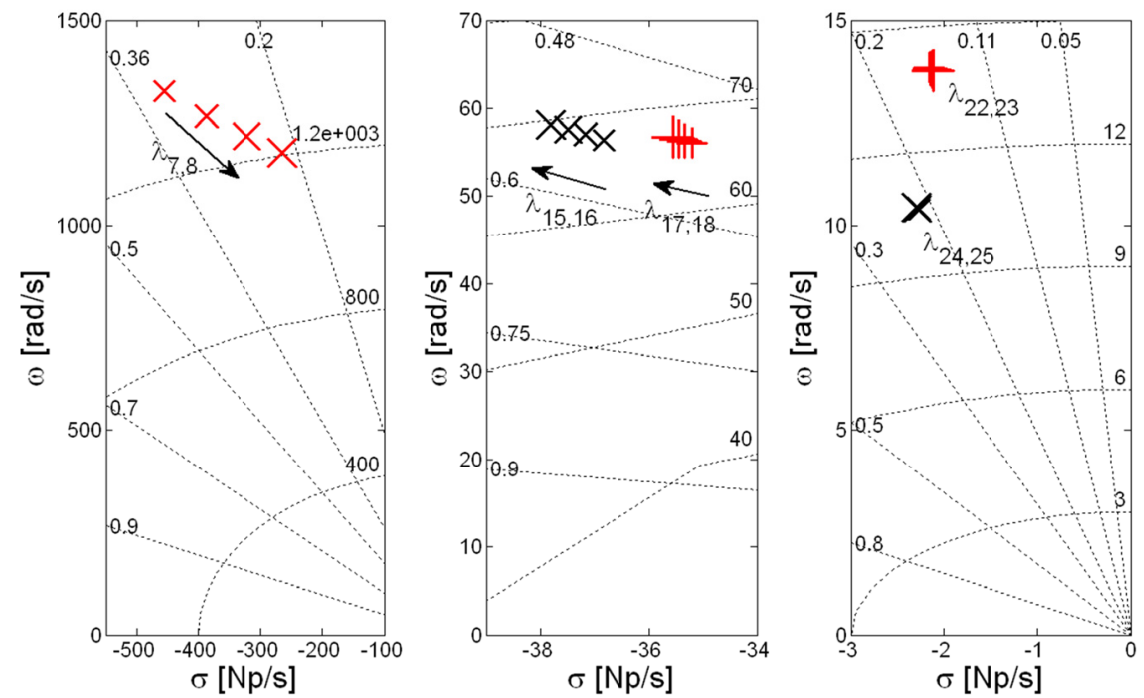

Figura 5.8 - Lugar geométrico dos modos oscilatórios dominantes em função do comprimento do alimentador $\boldsymbol{C}$ - Área A
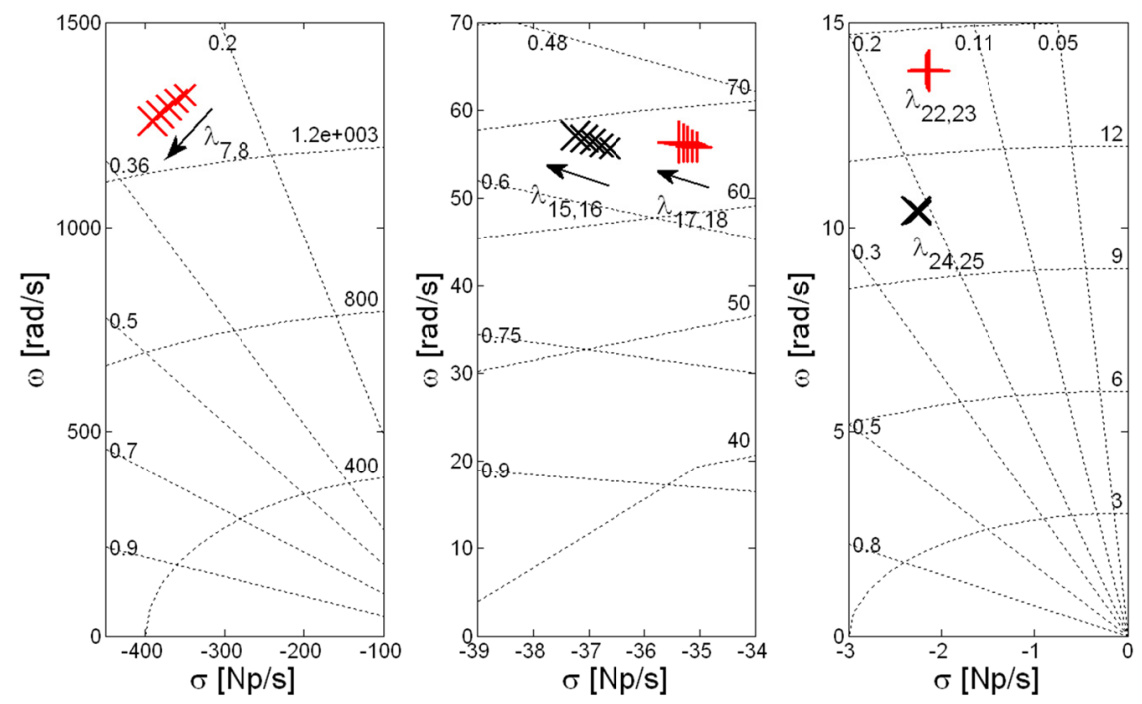

Figura 5.9 - Lugar geométrico dos modos oscilatórios dominantes em função da relação $\mathrm{R} / \mathrm{X}$ do alimentador $\boldsymbol{C}$ - Área $\mathrm{A}$

\footnotetext{
${ }^{19}$ A reatância de um alimentador está associada à disposição dos cabos na estrutura de suporte, consequentemente é praticamente independente do tipo de cabo utilizado. Já a resistência é inversamente proporcional à seção transversal do cabo.
} 
A análise das Figura 5.8 e Figura 5.9 mostra que os modos eletromecânicos do sistema $\left(\lambda_{22,23}\right.$ e $\left.\lambda_{24,25}\right)$, assim como os modos associados ao controle de potência ativa e reativa da $F E D_{2}\left(\lambda_{15,16}\right.$ e $\left.\lambda_{17,18}\right)$ são praticamente insensíveis às variações no comprimento e na relação $R / X$ do alimentador $\boldsymbol{C}$. O modo que apresenta a maior sensibilidade negativa ao incremento do comprimento é $\circ \lambda_{7,8}$, modo associado à malha de controle do PLL da $F E D_{2}$, com redução significativa do $\zeta$. Todavia, este modo apresenta sensibilidade positiva ao incremento da relação $R / X$.

\section{3 Área B}

A Figura 5.10 mostra o diagrama unifilar da segunda área sob estudo. Para este caso, define-se a operação nominal do sistema como aquela condição que apresenta: carga ativa nula (motor desligado), carga passiva coincidente máxima (43.5 kW), com $f p=0.92$ indutivo, estando a microturbina injetando potência ativa nominal $\left(P_{F E D 4}=1\right)$ e regulando a tensão na barra $6\left(V_{6}\right)$ em 1.0 pu. O GGD também regula a tensão na barra $5\left(V_{5}\right)$ em 1.0 pu. A interface da microturbina com a rede pode ser feita por um inversor com controle Droop ou Droop $+F$. Os dois casos são analisados a seguir.

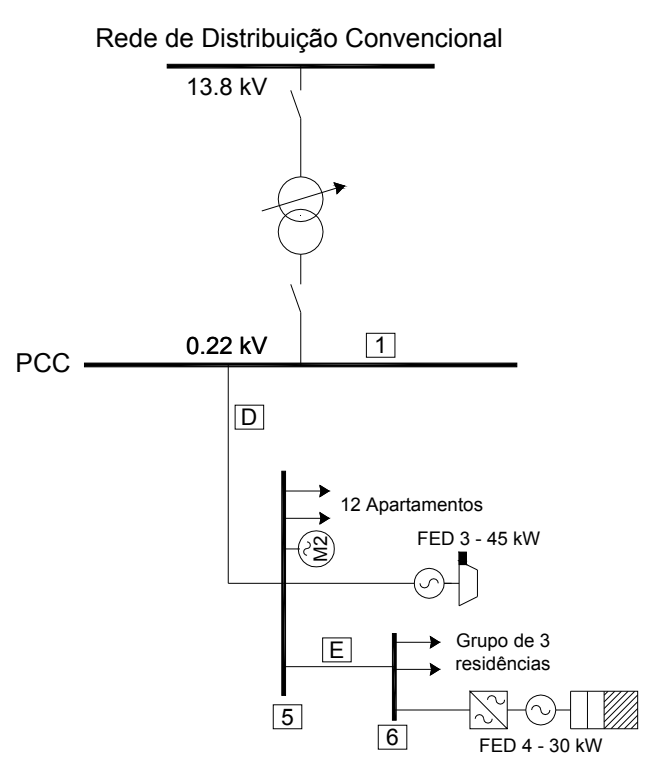

Figura 5.10 - Diagrama Unifilar da MG - Área B 


\subsubsection{Interface da FED 4 com Controle Droop}

A Tabela 5.2 mostra os principais autovalores do sistema correspondentes à operação nominal do sistema. Todos os autovalores têm parte real negativa, indicando uma condição de estabilidade para o ponto de operação considerado.

Tabela 5.2 - Autovalores da MG para operação nominal - Área B - Controle Droop

\begin{tabular}{cccc}
\hline Autovalor & $\sigma \pm j w$ & Autovalor & $\sigma \pm j w$ \\
\hline 1,2 & $-3882.8 \pm j 8637.3$ & 16 & -53.26 \\
3,4 & $-3657.3 \pm j 8131.9$ & 17,18 & $-26.425 \pm j 25.31$ \\
5,6 & $-1012.3 \pm j 1678.9$ & 19 & -33.68 \\
7,8 & $-927.81 \pm j 1595.0$ & 20,21 & $-10.354 \pm j 26.32$ \\
9,10 & $-884.59 \pm j 376.16$ & 22,23 & $-9.964 \pm j 0.850$ \\
11,12 & $-731.14 \pm j 363.55$ & 24,25 & $-3.93 \pm j 5.15$ \\
13,14 & $-111.51 \pm j 333.28$ & 26 & -0.16 \\
15 & -79.67 & & \\
\hline
\end{tabular}

A Figura 5.11 mostra os principais autovalores do sistema plotados no plano complexo.
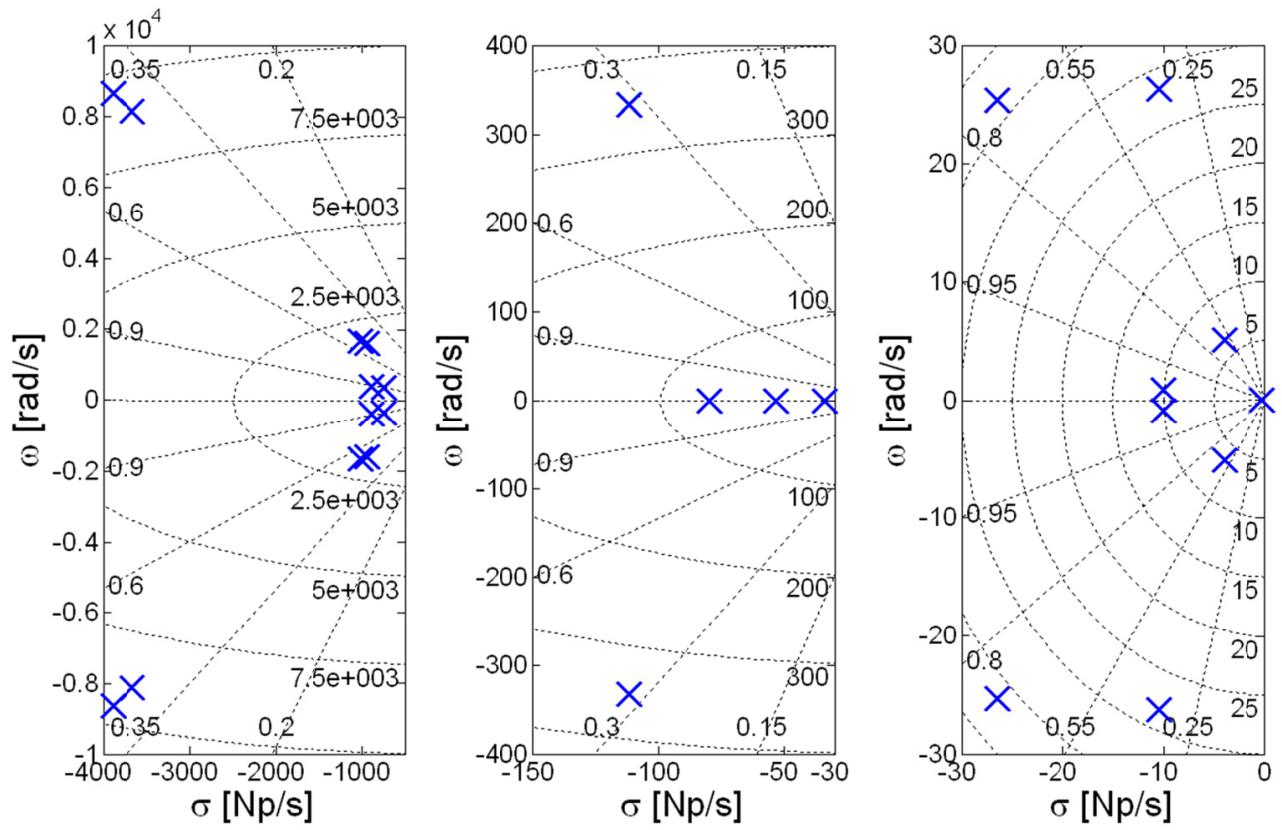

Figura 5.11 - Autovalores da MG para operação nominal - Área B - Controle Droop 
A análise da Figura 5.11 mostra um resultado muito interessante. Na presença da microturbina, acoplada à rede pelo inversor Droop, o modo eletromecânico associado à dinâmica do motor diesel e do regulador de velocidade do GGD $\left(\lambda_{20,21}\right)$ tem um aumento muito significativo do $\zeta$ e do $w$, passando de valores em torno de $\zeta=0.1$ e $w=2.0 \mathrm{~Hz}$ (para operação individual do GGD) a valores na faixa de $\zeta=0.37$ e $w=4.0 \mathrm{~Hz}$. De forma similar, o modo eletromecânico associado ao sistema de excitação do GGD $\left(\lambda_{24,25}\right)$ tem um incremento notável do $\zeta$, passando de valores em torno de $\zeta=0.2$ (para operação individual do GGD) para valores na faixa de $\zeta=0.61$, porém com redução do $w$ de $1.5 \mathrm{~Hz}$ para $0.8 \mathrm{~Hz}$. Entretanto, o modo associado ao controle de potência ativa do inversor com controle Droop $\left(\lambda_{17,18}\right)$, que era real para a operação individual do inversor, passa a ser complexo na presença do GGD, porém, com $\zeta$ elevado (igual a 0.72). Já o modo associado ao controle de potência reativa do inversor $\left(\lambda_{16}\right)$, se mantém real. Nota-se também que a constante de tempo do $\lambda_{26}$, associado ao controle secundário de frequência do GGD, se incrementa, indicando a necessidade de um tempo maior para restaurar a frequência do sistema ao seu valor programado.

Outra característica importante deste sistema é a perda significativa do $\zeta$ do modo associado às correntes transitórias de CC presentes nos enrolamentos do estator $\left(\lambda_{13,14}\right)$, passando de valores na faixa de 0.8 (para operação individual das FEDs) para valores em torno de 0.3. Este resultado mostra a importância de considerar os transitórios dos enrolamentos do estator $\left(\dot{\psi_{s d}}\right.$ e $\left.\dot{\psi_{s q}}\right)$ na modelagem da máquina síncrona quando FEDs acopladas à rede através de inversores com controle Droop estão presentes na MG.

Para validar estes resultados foi realizada uma simulação no domínio do tempo na plataforma Simulink® com modelos não lineares detalhados do sistema, para duas condições de operação diferentes. Para os dois casos, o distúrbio simulado corresponde a uma entrada de carga passiva de $20 \%$ na barra 5 . No primeiro caso, considera-se a operação conjunta do GGD e da microturbina, enquanto no segundo caso a microturbina é totalmente desligada do sistema. O resultado se mostra na Figura 5.12. 
Como a análise modal mostrou, as oscilações eletromecânicas são muito mais amortecidas e de maior frequência (aproximadamente o dobro) para o Caso 1 do que para o Caso 2. Observa-se também que a frequência é reestabelecida ao seu valor programado mais rapidamente no Caso 2 , como também previsto pela análise modal.

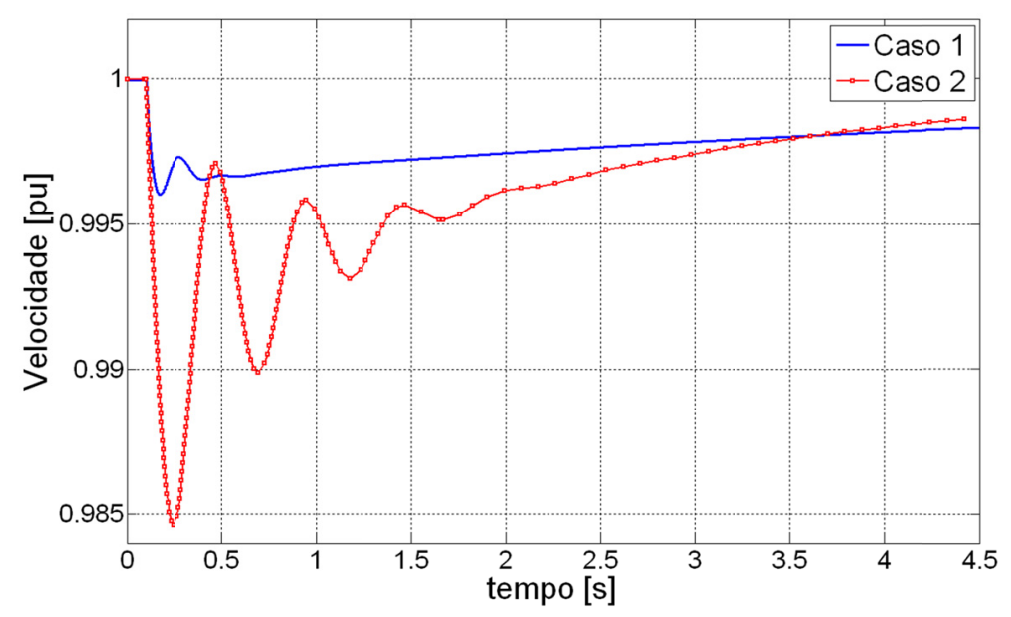

Figura 5.12 - Resposta transitória de velocidade - $\boldsymbol{F} \boldsymbol{E} \boldsymbol{D}_{3}$

Para analisar a influência do $P_{F E D 4}$, assim como do nível de tensão da barra 6 (controlado através da injeção ou absorção de reativos por parte da microturbina $Q_{F E D 4}$ ) na estabilidade dinâmica do sistema, uma análise de sensibilidade foi realizada. Os lugares geométricos dos modos que maiores variações apresentaram se mostra na Figura 5.13 e Figura 5.14.

A análise das figuras revela que os modos eletromecânicos são pouco sensíveis ao ponto de operação da microturbina, observando-se uma discreta variação do $\zeta$, especialmente no $\lambda_{20,21}$. A frequência de oscilação dos modos também é pouco afetada pelas variações no $P_{F E D 4}$ e no $V_{6}$. Este resultado importante mostra que diferentemente da interação entre um GGD e um inversor com controle P-Q, a interação de um GGD com um inversor com controle Droop aumenta drasticamente o $\zeta$ dos modos eletromecânicos do GGD, independentemente da potência ativa ou reativa injetada pela FED acoplada ao inversor. 

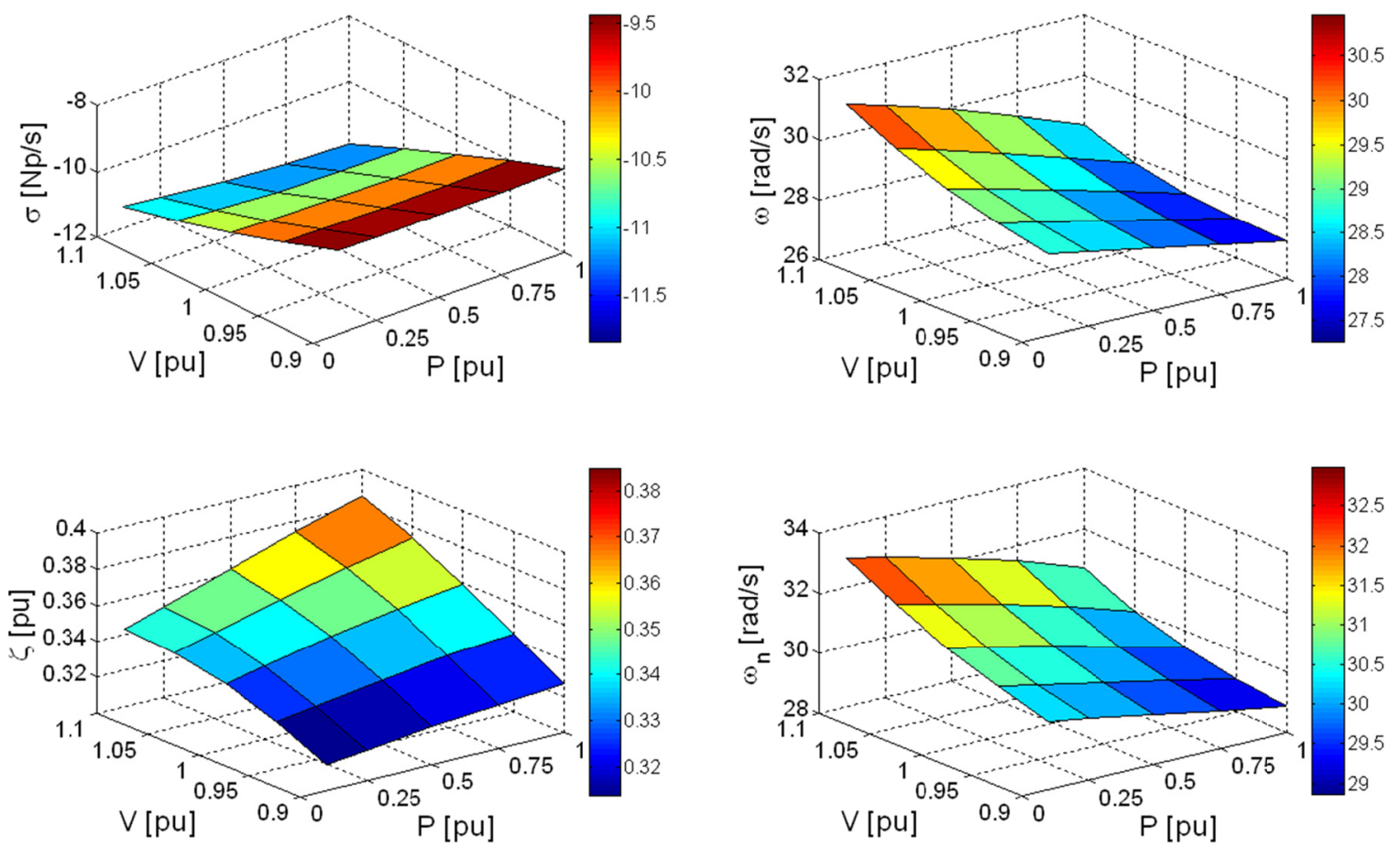

Figura 5.13 - Lugar geométrico do $\lambda_{20,21}$ para variações no $\boldsymbol{P}_{F E D 4}$ e $\boldsymbol{V}_{6}$ - Área B
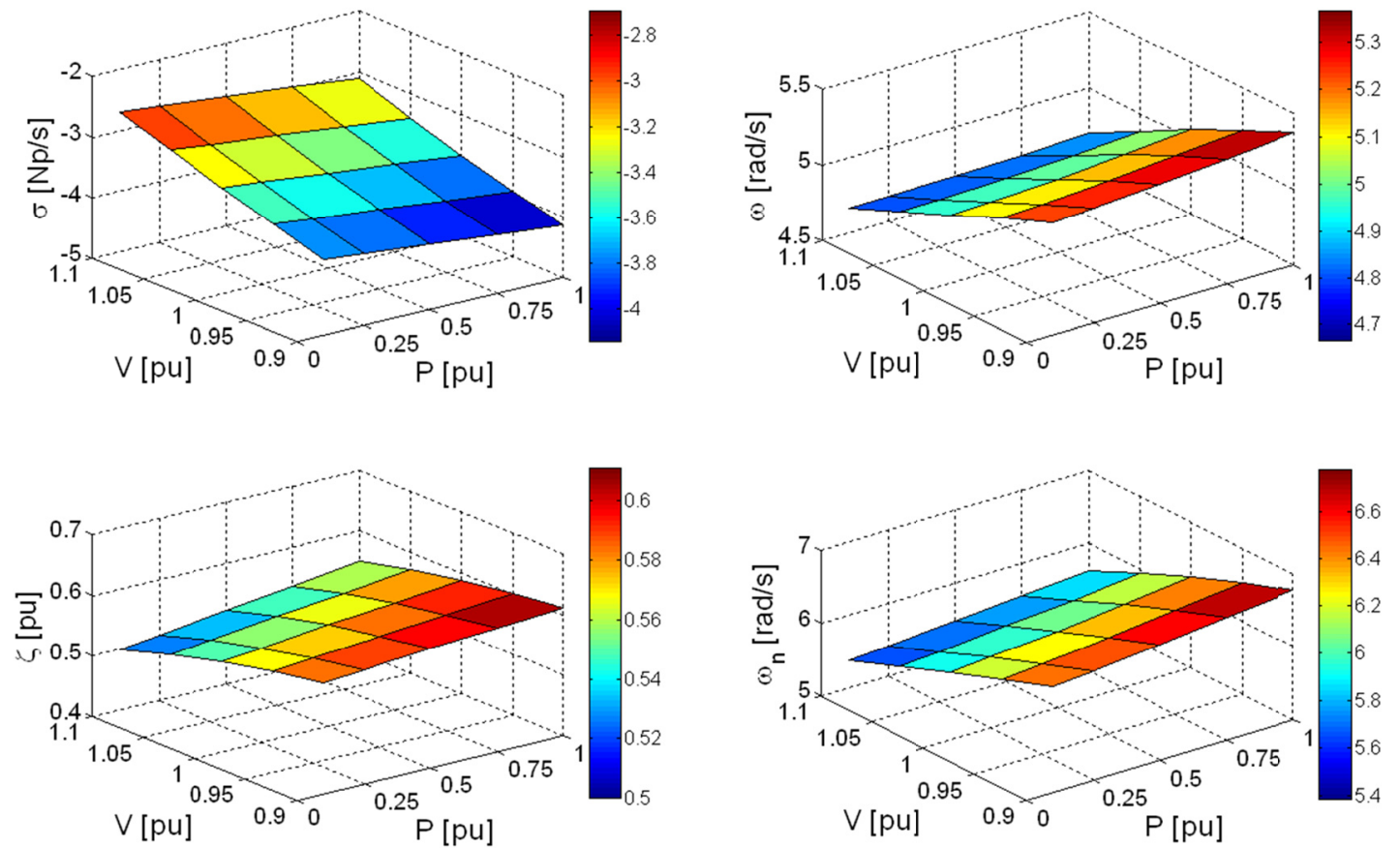

Figura 5.14 - Lugar geométrico do $\lambda_{24,25}$ para variações no $\boldsymbol{P}_{F E D 4}$ e $\boldsymbol{V}_{6}$ - Área B 
A seguir se analisa o comportamento dinâmico do sistema na presença do motor. A Tabela 5.3 mostra os principais autovalores do sistema quando o motor de indução de $15 \mathrm{~kW}$ é conectado na barra 5 da MG, com torque mecânico nominal aplicado no seu eixo. A carga passiva coincidente é mantida em $43.5 \mathrm{~kW}-\mathrm{fp}=0.92$. Como pode se observar, todos os autovalores têm parte real negativa, indicando uma condição de estabilidade para o ponto de operação considerado.

Tabela 5.3 - Autovalores da MG na presença do MI - Área B - Controle Droop

\begin{tabular}{cccc}
\hline Autovalor & $\sigma \pm j w$ & Autovalor & $\sigma \pm j w$ \\
\hline 1,2 & $-3891.2 \pm j 8735.0$ & 20 & -60.929 \\
3,4 & $-3671.1 \pm j 8228.9$ & 21 & -38.042 \\
5,6 & $-999.30 \pm j 1671.0$ & 22 & -33.758 \\
7,8 & $-915.75 \pm j 1583.4$ & 23,24 & $-10.809 \pm j 29.48$ \\
9,10 & $-884.59 \pm j 375.2$ & 25,26 & $-24.248 \pm j 18.02$ \\
11,12 & $-758.38 \pm j 366.56$ & 27 & -12.993 \\
13,14 & $-68.208 \pm j 366.82$ & 28 & -8.712 \\
15,16 & $-116.42 \pm j 331.03$ & 29,30 & $-3.170 \pm j 5.408$ \\
17,18 & $-17.853 \pm j 99.904$ & 31 & -0.165 \\
19 & -82.14 & & \\
\hline
\end{tabular}

A Figura 5.15 mostra os principais autovalores do sistema plotados no plano complexo. Como se pode observar, a presença do motor de indução não afeta a estabilidade dinâmica das FEDs existentes na MG, mantendo-se praticamente constante o $\zeta$ e $w$ de todos os modos do sistema. Os modos oscilatórios associados ao motor de indução $\left(\lambda_{13,14}, \lambda_{17,18}\right)$ apresentam valores similares aos obtidos no Capitulo 4 para conexão direta do motor de indução nas FEDs.

Para analisar o impacto da sobrecarga do MI na estabilidade dinâmica do sistema, uma análise de sensibilidade dos autovalores ao torque mecânico aplicado ao motor foi realizada $\mathrm{O}$ valor de torque pré-distúrbio foi variado entre $100 \%$ e $250 \%$, em passos de 50\%. A Figura 5.16 mostra o lugar geométrico dos modos dominantes que maiores variações apresentaram. 

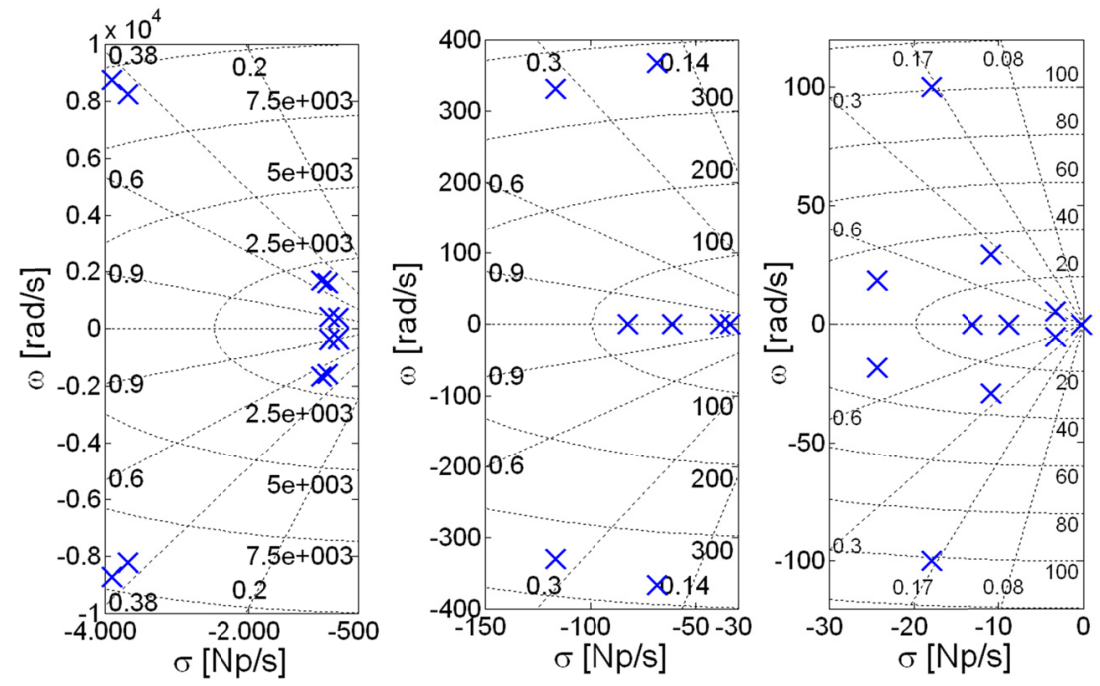

Figura 5.15 - Autovalores da MG na presença do MI - Área B - Controle Droop

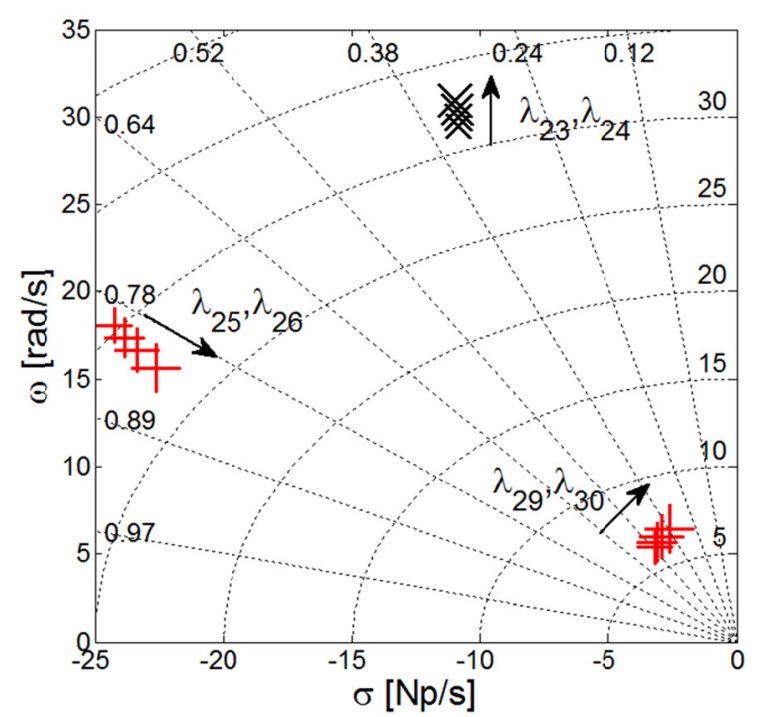

Figura 5.16 - Lugar geométrico dos modos oscilatórios dominantes para variações no Torque

A análise da Figura 5.16 mostra uma sensibilidade negativa do modo eletromecânico $\lambda_{29,30}$, associado ao sistema de excitação do GGD, com perda do seu $\zeta$ e leve incremento do $w_{n}$. Pelo contrário, o modo eletromecânico $\lambda_{23,24}$, associado à dinâmica do motor diesel e do regulador de velocidade, mostra uma sensibilidade positiva, com incremento do $w_{n}$ e uma ligeira redução do $\zeta$. Já o modo $\lambda_{25,26}$, associado ao controle de potência ativa do inversor com controle Droop, mostra uma sensibilidade negativa, com perda de $w_{n}$ e $\zeta$ praticamente constante. 
Ao comparar estes resultados com os obtidos para operação individual do GGD (Secção 4.1.2), se observa que a presença da FED com controle Droop no sistema, minimiza o impacto negativo da sobrecarga do motor no $\zeta$ do modo eletromecânico do GGD associado ao sistema de excitação.

A seguir se analisa a sensibilidade dos autovalores às variações no comprimento dos cabos e na relação $R / X$ dos mesmos. A Figura 5.17 mostra o lugar geométrico dos modos oscilatórios dominantes do sistema em função da distância elétrica entre as FEDs (i.e., em função do comprimento do alimentador $\boldsymbol{E}$ ), para operação nominal do sistema (sem a presença do MI). A distância foi variada entre $0 \mathrm{~m}$ e $200 \mathrm{~m}$, em passos de $50 \mathrm{~m}$, mantendo constante a bitola do cabo do alimentador $\boldsymbol{E}$. Por outra parte, a Figura 5.18 mostra o lugar geométrico dos modos oscilatórios dominantes do sistema em função da relação $R / X$ do alimentador $\boldsymbol{E}$. A relação foi variada entre 0.5 e 2.5 , em passos de 0.5 , mantendo constante a reatância do alimentador e alterando unicamente sua resistência.

A análise da Figura 5.17 mostra que o modo eletromecânico $\lambda_{20,21}$, associado à dinâmica do motor diesel e do regulador de velocidade do GGD, é muito sensível às variações no comprimento do alimentador $\boldsymbol{E}$, com perda significativa do $\zeta$ à medida que aumenta a distância elétrica entre as FEDs, entretanto, mostra-se pouco sensível às variações na relação $R / X$. Consequentemente, conclui-se que o $\zeta$ do modo $\lambda_{20,21}$ é, prioritariamente, função da reatância do alimentador e não da sua resistência. Pelo contrário, o segundo modo eletromecânico $\left(\lambda_{24,25}\right)$, associado ao sistema de excitação do GGD, mostrasse insensível às variações nos parâmetros do alimentador $\boldsymbol{E}$.

Por outra parte, nota-se que o modo relacionado ao controle de potência ativa do inversor com controle Droop $\left(\lambda_{17,18}\right)$ é positivamente sensível ao incremento da distância entre as FEDs, com aumento do $\zeta$, embora, seu $w$ apresente uma ligeira redução. Observa-se também que a relação $R / X$ do alimentador não altera $0 \zeta$ do modo, unicamente seu $w$.

É importante observar que o $\lambda_{13,14}$, associado ao transitório elétrico no estator do GGD, é fortemente afetado pelas variações nos parâmetros elétricos do alimentador, 
com perda significativa do $\zeta$ à medida que as FEDs se aproximam eletricamente. Da análise das figuras acima é possível inferir que este comportamento deve-se principalmente à perda de resistência elétrica do alimentador, mais do que a sua reatância, e mostra novamente a necessidade de considerar os transitórios do estator $\left(\dot{\psi_{s d}} e \dot{\psi_{s q}}\right)$ na modelagem da máquina síncrona, quando operada em paralelo com inversores com controle Droop.
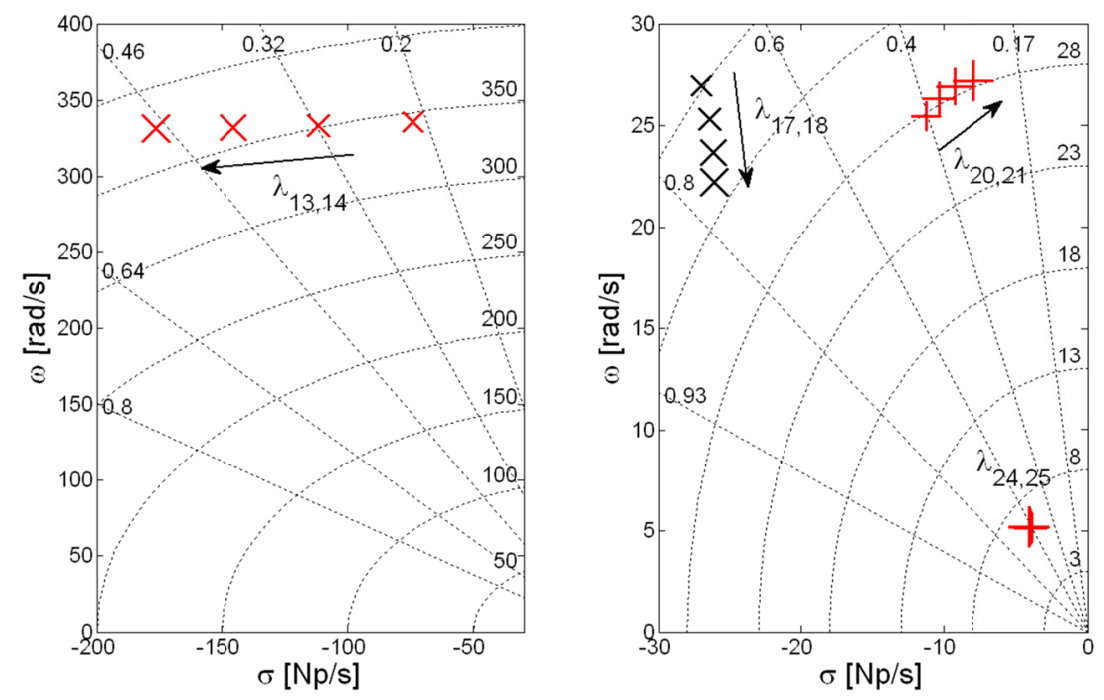

Figura 5.17 - Lugar geométrico dos modos oscilatórios dominantes em função do comprimento do alimentador $\boldsymbol{E}$ - Área B
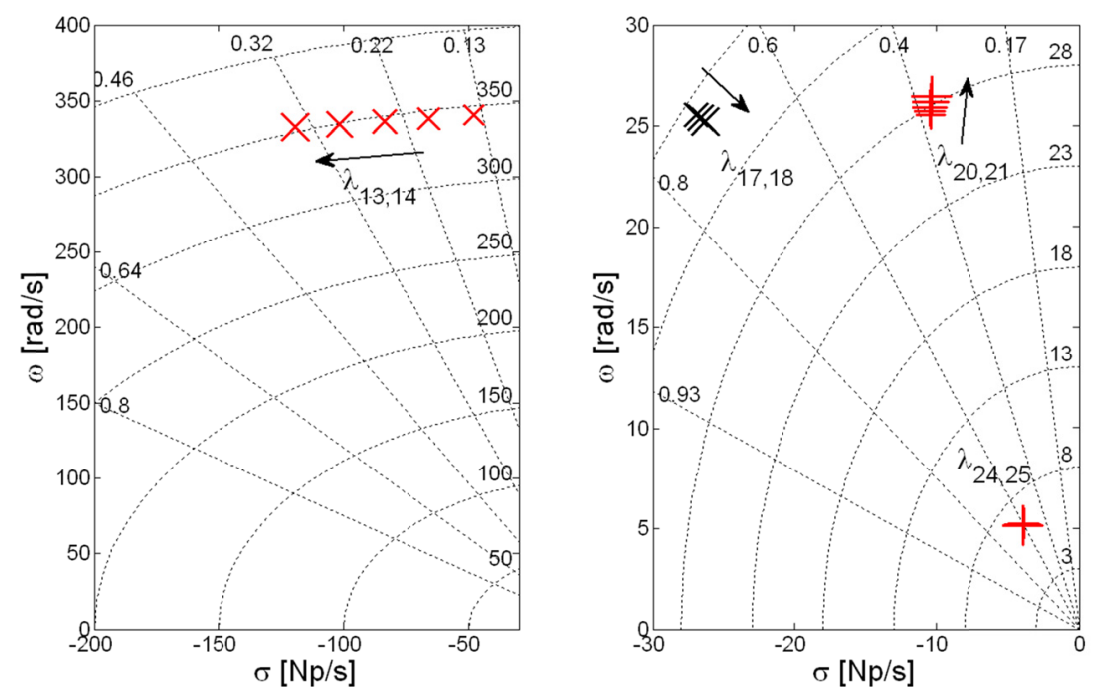

Figura 5.18 - Lugar geométrico dos modos oscilatórios dominantes em função da relação $\mathrm{R} / \mathrm{X}$ do alimentador $\boldsymbol{E}$ - Área B 
Estes resultados também são muito importantes do ponto de vista de projeto de uma MG, pois mostram que em sistemas onde GGDs e FEDs (acopladas à rede através de inversores com controle Droop) operam em paralelo, é aconselhável minimizar a distância elétrica entre este tipo de fontes, de forma a garantir a minimização dos transitórios eletromecânicos dos GGDs, embora o transitório das correntes de CC (com constantes de tempo muito menores) seja menos amortecido.

Por outra parte, estes resultados também mostram que na ausência de GGDs no sistema, o afastamento elétrico entre as FEDs é positivo para a estabilidade dinâmica do sistema, em outras palavras, a geração dispersa (elemento chave de uma MG) através de FEDs acopladas à rede através de inversores com controle Droop, favorece a estabilidade do sistema. Finalmente, estes resultados também revelam que em aplicações práticas, onde a indutância dos alimentadores de BT é praticamente constante, a escolha de uma determinada seção transversal de cabo tem pouco impacto nos modos eletromecânicos do sistema, devendo sua escolha obedecer a outro tipo de requisito, como nível de perdas ou regulação de tensão.

No caso do controle droop, um assunto muito investigado na literatura especializada é a influência do valor do droop de potência ativa (parâmetro $R_{p-D}$ ) na estabilidade dinâmica do sistema. Em todas as análises realizadas até aqui, tem se utilizado um valor de droop de $0.8 \%$, equivalente a uma variação de frequência de $0.5 \mathrm{~Hz}$ entre carga nominal e em vazio, valor fornecido pelo fabricante do inversor. Contudo, considera-se importante realizar uma análise de sensibilidade dos autovalores a este parâmetro. A Figura 5.19 mostra o lugar geométrico dos modos oscilatórios dominantes do sistema para operação nominal da MG em função do parâmetro $R_{p-D}$ do inversor associado à $F E D_{4}$. O valor de $R_{p-D}$ foi variado entre $0.8 \%$ e $1.6 \%$, em passo de $0.2 \%$.

Como observado no gráfico, nota-se que o incremento de $R_{p-D}$, até $1 \%$ aproximadamente, contribui ao aumento do $\zeta$ do modo eletromecânico $\lambda_{20,21}$, todavia, valores de $R_{p-D}$ acima de $1 \%$ unicamente deixam o modo mais lento, sem contribuição favorável para o incremento de $\zeta$. Por outra parte, qualquer aumento de $R_{p-D}$ afeta negativamente o $\lambda_{17,18}$, reduzindo drasticamente seu $\zeta$. Um valor de $R_{p-D}$ entre $0.8 \%$ e $1 \%$ apresenta os melhores resultados para as duas FEDs. Já os 
modos restantes $\left(\lambda_{13,14}\right.$ e $\left.\lambda_{24,25}\right)$ se mostram insensíveis às variações no valor do droop.
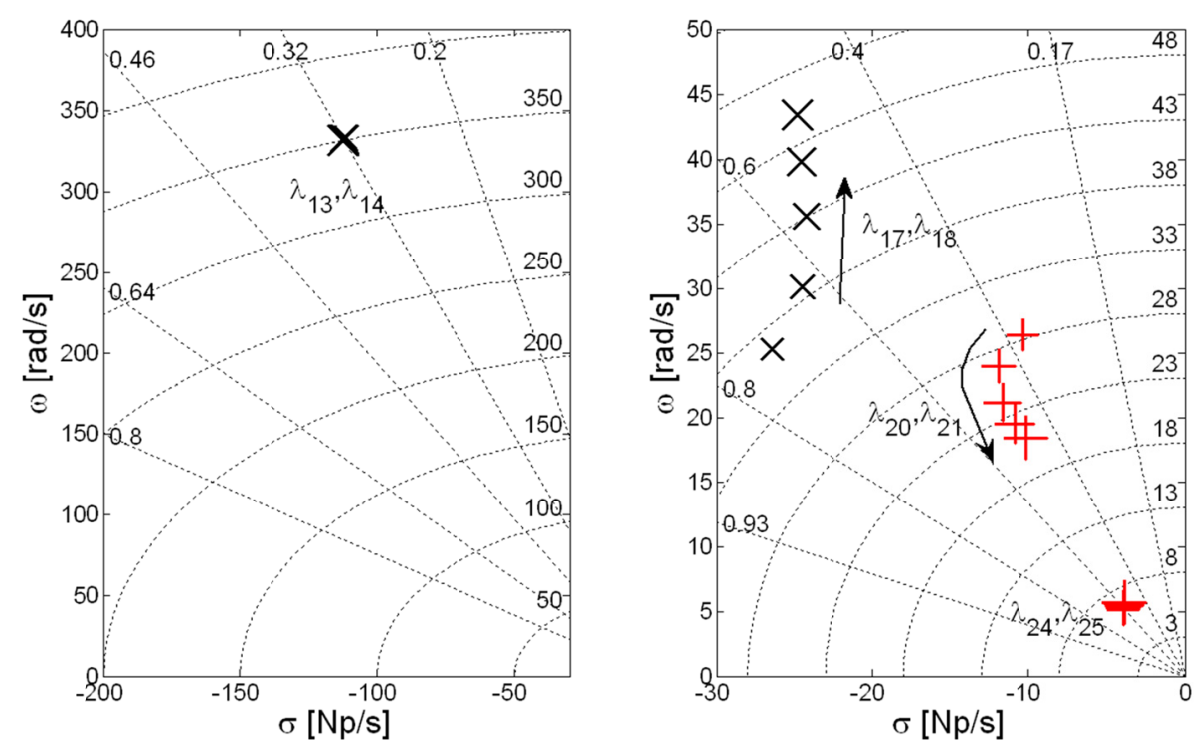

Figura 5.19 - Lugar geométrico dos modos oscilatórios dominantes em função do parâmetro $\boldsymbol{R}_{\boldsymbol{p}-\boldsymbol{D}}$ - Área B

É importante observar que, embora valores baixos de $R_{p-D}$ sejam desejáveis do ponto de vista da estabilidade dinâmica, o menor valor de droop do inversor (se comparado ao droop do GGD), implica que durante uma aceitação de carga do sistema, a partilha de potência ativa entre as FEDs durante o transitório é diferente, sendo a FED acoplada ao inversor Droop aquela que maior potência fornece à carga durante o transitório, até a frequência ser restabelecida ao seu valor programado. Consequentemente, cuidados especiais devem ser tomados na calibração dos droops das diferentes fontes, de forma a preservar uma sobrecarga inadmissível das mesmas.

A Figura 5.20 mostra a resposta transitória de potência ativa das FEDs da Área B, calculada através de simulação computacional no domínio do tempo na plataforma Simulink $\circledast$, com modelos não lineares detalhados do sistema. O distúrbio simulado corresponde a uma entrada de carga passiva de $25 \%$ na barra 5 , com valores de Droop de $4 \%$ e $0.8 \%$ para a $F E D_{3}$ e $F E D_{4}$ respectivamente. Os valores de potência 
ativa pré-distúrbio são iguais para as duas fontes e correspondem a um valor de $0.62 \mathrm{pu}$

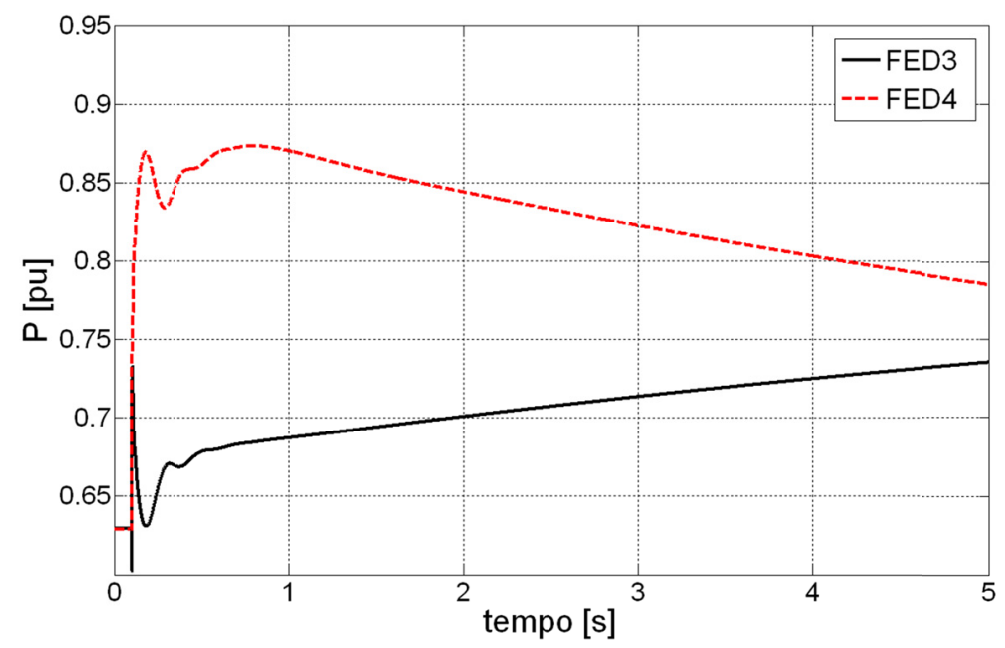

Figura 5.20 - Resposta transitória de potência ativa - Área B

Como esperado, a figura acima mostra que durante o transitório a $F E D_{4}$ fornece a maior parcela de potência ativa à carga, chegando a um valor máximo de $0.87 \mathrm{pu}$. À medida que frequência é restabelecida, a partilha de potência ativa torna-se mais equitativa entre as duas FEDs

\subsubsection{Interface da $\mathrm{FED}_{4}$ com Controle Droop+F}

A Tabela 5.4 mostra os principais autovalores do sistema correspondentes à operação nominal do sistema. Todos os autovalores têm parte real negativa, indicando uma condição de estabilidade para o ponto de operação considerado.

Ao comparar a Tabela 5.4 com a Tabela 5.2, percebe-se que, com exceção do $\lambda_{26}$, o controle secundário de frequência do Controle Droop $+F$ tem um impacto mínimo na posição dos autovalores do sistema. O modo que apresenta a maior sensibilidade negativa é o modo eletromecânico $\lambda_{20,21}$, com redução do $\zeta$ de 0.37 para 0.33 . Em 
contrapartida, a redução significativa da constante de tempo do $\lambda_{26}$ indica que a restauração da frequência ao valor programado é muito mais rápida.

Tabela 5.4-Autovalores da MG para operação nominal - Área B - Controle Droop+F

\begin{tabular}{cccc}
\hline Autovalor & $\sigma \pm j w$ & Autovalor & $\sigma \pm j w$ \\
\hline 1,2 & $-3882.8 \pm \mathrm{j} 8637.3$ & 16 & -54.210 \\
3,4 & $-3657.3 \pm \mathrm{j} 8132.0$ & 17,18 & $-26.277 \pm \mathrm{j} 23.52$ \\
5,6 & $-1012.3 \pm \mathrm{j} 1678.9$ & 19 & -33.586 \\
7,8 & $-927.82 \pm \mathrm{j} 1594.9$ & 20,21 & $-10.15 \pm \mathrm{j} 28.665$ \\
9,10 & $-884.59 \pm \mathrm{j} 376.13$ & 22,23 & $-10.51 \pm \mathrm{j} 1.304$ \\
11,12 & $-731.1 \pm \mathrm{j} 363.52$ & 24,25 & $-3.656 \pm \mathrm{j} 4.925$ \\
13,14 & $-111.54 \pm \mathrm{j} 333.29$ & 26 & -0.821 \\
15 & -79.346 & & \\
\hline
\end{tabular}

Para validar estes resultados, duas simulações no domínio do tempo na plataforma Simulink® foram realizadas com modelos não lineares detalhados do sistema. No primeiro caso, a interface da $F E D_{4}$ com a rede se dá através de um inversor com Controle Droop. Já no segundo caso, a interface da $F E D_{4}$ se dá através de um inversor com Controle Droop $+F$. Para os dois casos o distúrbio simulado corresponde a uma entrada de carga passiva de $20 \%$ na barra 5 . A resposta transitória de velocidade da $F E D_{3}$ se mostra na Figura 5.21.

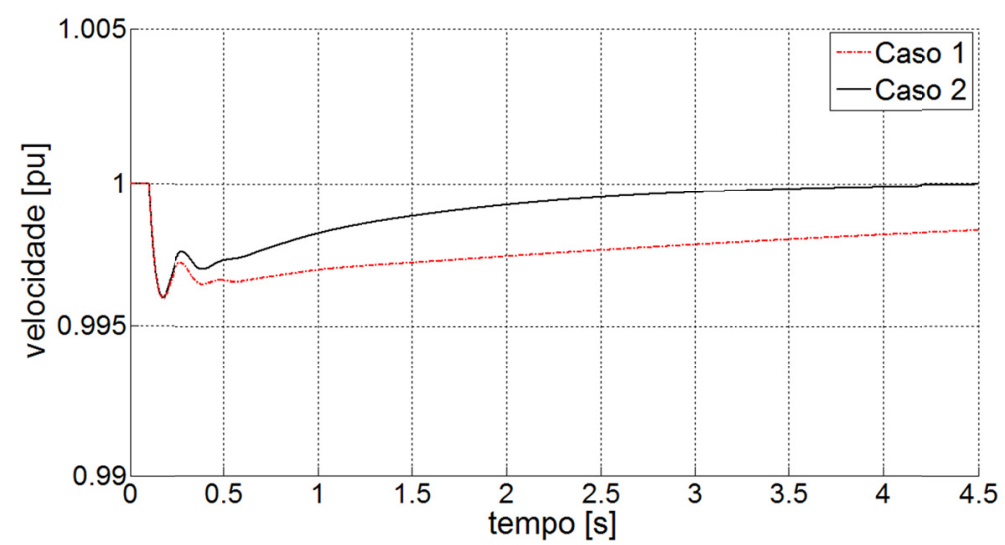

Figura 5.21 - Resposta transitória de velocidade $\boldsymbol{F E} \boldsymbol{D}_{3}-$ Controle Droop+F - Área B 
Como mostrado pela análise modal, observa-se que a restauração de frequência ao seu valor programado é muito mais rápida no Caso2, sendo que tanto a amplitude quanto a frequência de oscilação da velocidade durante o transitório eletromecânico é similar para os dois casos. Este resultado mostra que na presença do Controle Droop $+F$ é possível restabelecer rapidamente a frequência da MG ao seu valor programado, sem prejudicar a estabilidade do sistema.

\section{4 Área C}

A Figura 5.22 mostra o diagrama unifilar da terceira área sob estudo. Para este caso, define-se a operação nominal do sistema como aquela condição que apresenta carga passiva coincidente máxima $(20 \mathrm{~kW}) \mathrm{com} \mathrm{fp}=0.92$ indutivo, estando a turbina eólica injetando potência ativa nominal $\left(P_{F E D 6}=1\right) \mathrm{com} \mathrm{fp=1.0}\left(Q_{F E D 6}=0\right)$ e a microturbina regulando a tensão na barra $8\left(V_{8}\right)$ em $1.0 \mathrm{pu}$. A interface da microturbina com a rede pode ser feita por um inversor com controle Droop ou Droop+F. Os dois casos são analisados a seguir.

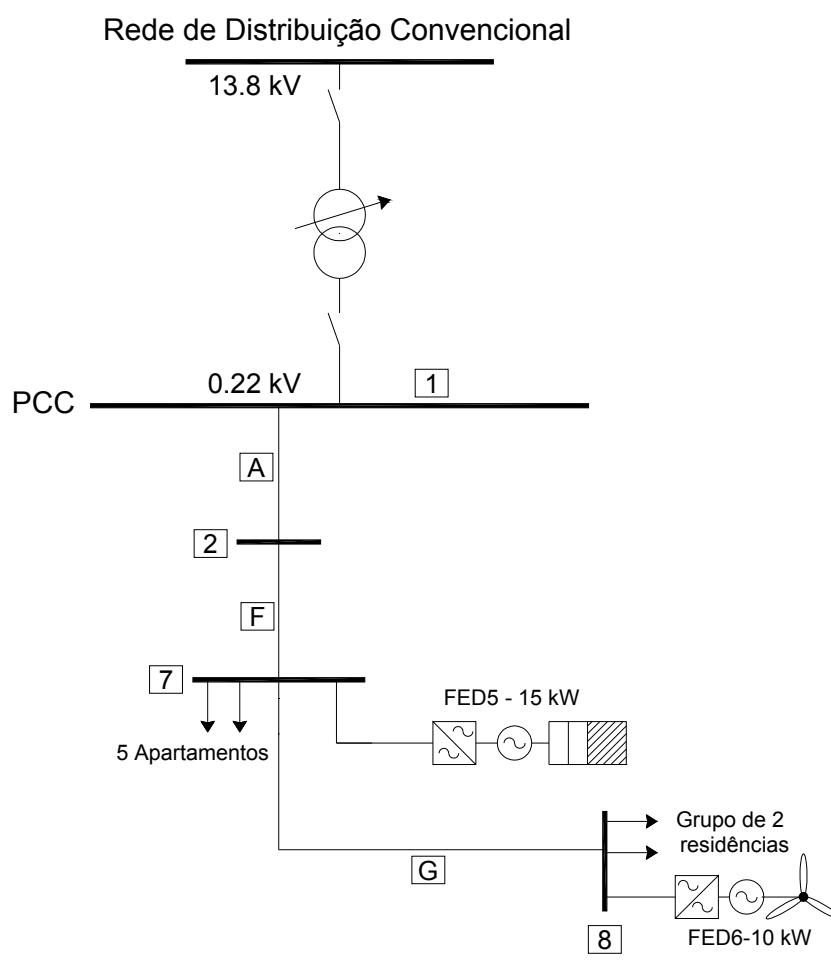

Figura 5.22 - Diagrama Unifilar da MG - Área C 


\subsubsection{Interface da FED 5 com Controle Droop}

A Tabela 5.5 mostra os principais autovalores do sistema correspondente à operação nominal do sistema. Todos os autovalores têm parte real negativa, indicando uma condição de estabilidade para o ponto de operação considerado.

Tabela 5.5 - Autovalores da MG para operação nominal - Área C - Controle Droop

\begin{tabular}{cccc}
\hline Autovalor & $\sigma \pm j w$ & Autovalor & $\sigma \pm j w$ \\
\hline 1,2 & $-3588.6 \pm j 9060.9$ & 17,18 & $-884.74 \pm j 378.6$ \\
3,4 & $-3581.0 \pm j 8615.9$ & 19,20 & $-424.7 \pm j 726.16$ \\
5,6 & $-4580.3 \pm j 7438.4$ & 21,22 & $-673.96 \pm j 349.4$ \\
7,8 & $-4244.1 \pm j 6824.6$ & 23,24 & $-39.01 \pm j 55.27$ \\
9,10 & $-1016.3 \pm j 2065.7$ & 25,26 & $-37.88 \pm j 55.98$ \\
11,12 & $-1394.6 \pm j 1447.2$ & 27 & -43.171 \\
13,14 & $-576.18 \pm j 1704.2$ & 28 & -39.582 \\
15,16 & $-904.19 \pm j 1375.0$ & & \\
\hline
\end{tabular}

A Figura 5.23 mostra os principais autovalores do sistema plotados no plano complexo.
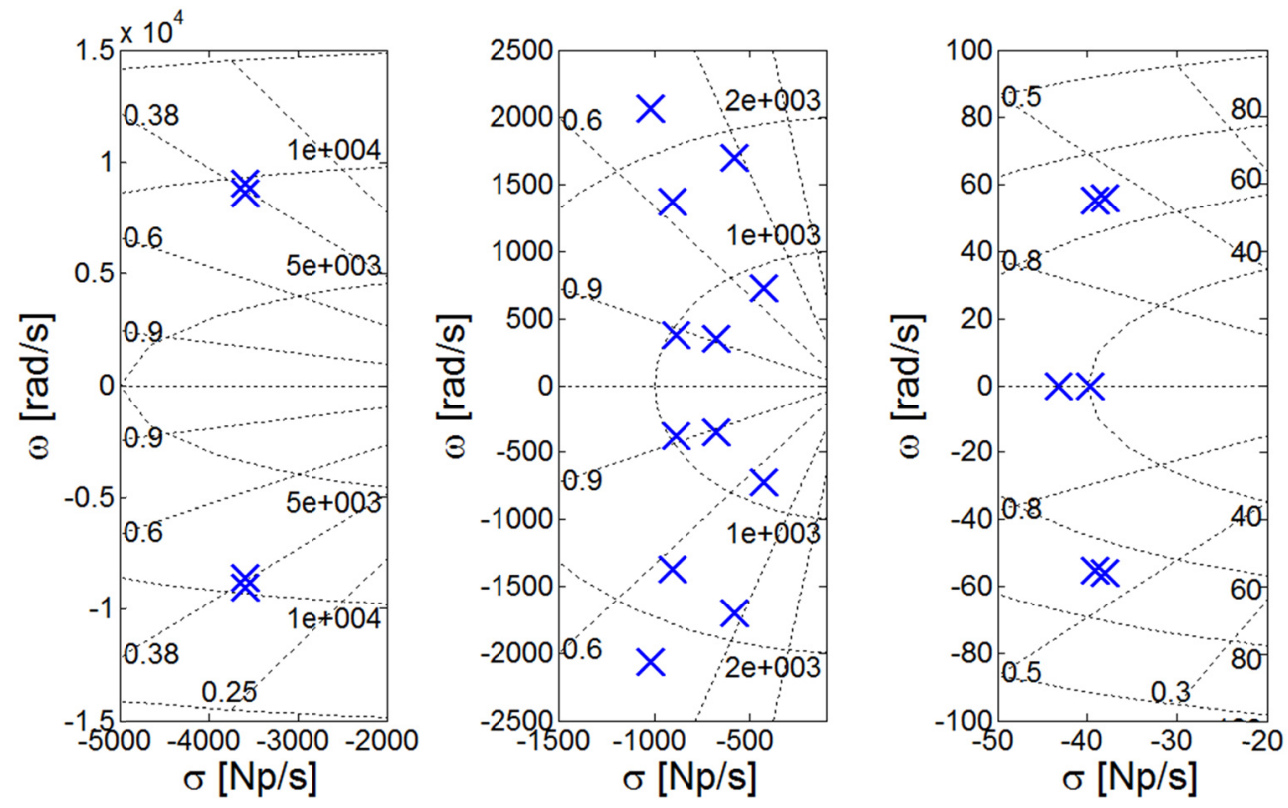

Figura 5.23- Autovalores da MG para operação nominal - Área C - Controle Droop 
Ao analisar a Figura 5.23 nota-se que os modos associados ao controle de potência ativa e reativa do inversor com controle Droop $\left(\lambda_{27,28}\right)$, que na presença do GGD eram complexos (Área B), voltam a ser reais, com valores similares aos obtidos para a operação individual do inversor. Já os modos associados ao controle de potência ativa e reativa do inversor com controle P-Q $\left(\lambda_{23,24}\right.$ e $\left.\lambda_{25,26}\right)$ continuam sendo complexos, porém, com valores também similares aos registrados para operação individual do inversor. Por outra parte, nota-se que neste caso a malha do PLL do inversor $\mathrm{P}-\mathrm{Q}$ não encontra dificuldade em rastrear o ângulo de fase da tensão, apresentando o modo $\lambda_{19,20}$ um $\zeta=0.5$, valor muito superior ao $\zeta=0.3$ obtido na presença do GGD (Área A). Em contrapartida, um dos modos associados à malha de tensão do controle Droop $\left(\lambda_{13,14}\right)$ tem uma redução considerável do $\zeta$, de 0.55 (para operação individual do inversor) para 0.32. Os modos restantes do sistema apresentam pouca variação quando comparados à operação individual dos inversores.

Para analisar a influência do $P_{F E D 6}$ e $Q_{F E D 6}$ injetados no sistema, uma análise de sensibilidade foi realizada. Os lugares geométricos dos modos que maiores variações apresentaram se mostram nas figuras a seguir.
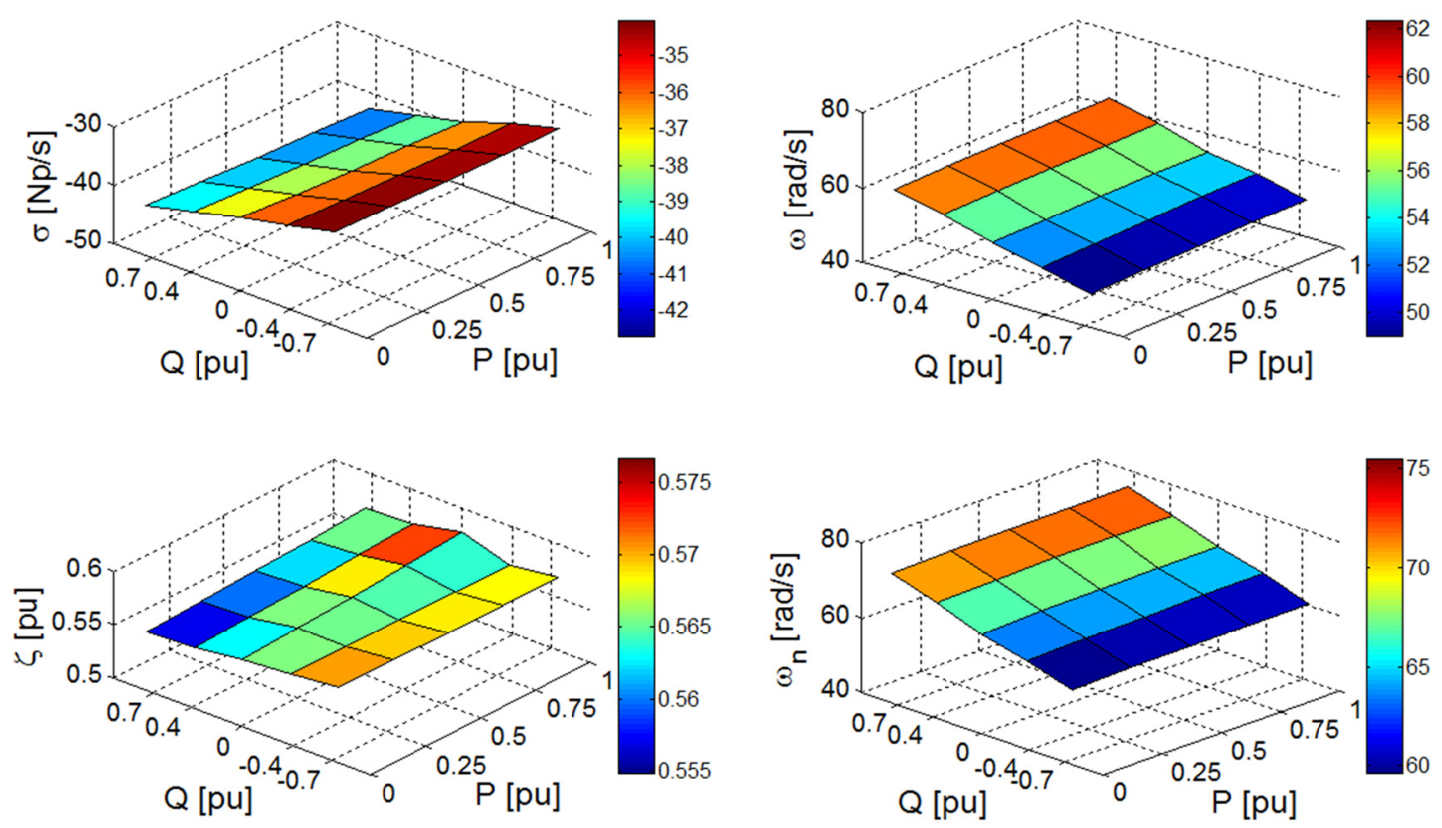

Figura 5.24 - Lugar geométrico do $\lambda_{23,24}$ para variações no $\boldsymbol{P}_{F E D 6}$ e $\boldsymbol{Q}_{F E D 6}$ - Área C 

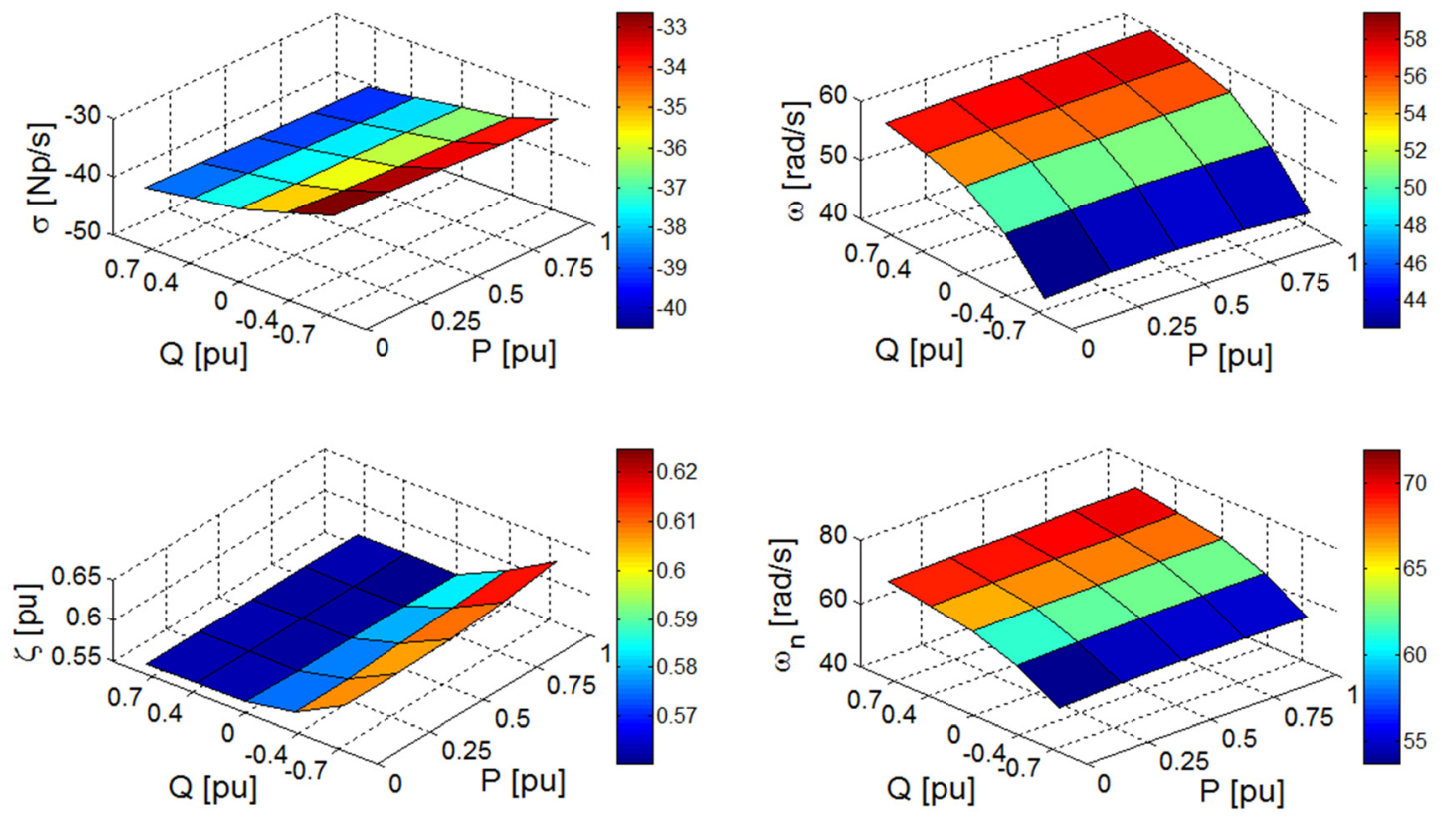

Figura 5.25 - Lugar geométrico do $\lambda_{25,26}$ para variações no $\boldsymbol{P}_{F E D 6}$ e $\boldsymbol{Q}_{F E D 6}$ - Área C

A análise das figuras mostra que os modos $\lambda_{23,24}$ e $\lambda_{25,26}$, associados à potência ativa e reativa do controle $P-Q$ respectivamente, são minimamente sensíveis aos valores de $P_{F E D 6}$ e $Q_{F E D 6}$ injetados na rede. A maior sensibilidade mostra $\circ \lambda_{23,24}$, registrando-se uma perda de $\zeta$ devido à injeção de reativos no sistema ( $Q$ positivo).

A Figura 5.26 mostra o lugar geométrico dos modos dominantes do sistema em função da distância elétrica entre as FEDs (i.e., em função do comprimento do alimentador $\mathbf{G}$ ) para operação nominal do sistema. A distância foi variada entre $0 \mathrm{~m}$ e $200 \mathrm{~m}$, em passos de $50 \mathrm{~m}$, mantendo constante a bitola do condutor. Já a Figura 5.27 mostra o lugar geométrico dos modos dominantes em função da relação $R / X$ do alimentador $\boldsymbol{G}$. A relação foi variada entre 0.5 e 2.5 em passos de 0.5 , mantendo constante a reatância do alimentador e alterando unicamente sua resistência.

A análise da Figura 5.26 e Figura 5.27 mostra que os modos $\lambda_{23,24}$ e $\lambda_{25,26}$, associados à potência ativa e reativa do controle $P-Q$, são pouco sensíveis às variações nos parâmetros do alimentador. Menos sensíveis ainda, são os modos associados ao controle de potência ativa e reativa do controle Droop. Todavia, os modos $\lambda_{13,14} \mathrm{e} \lambda_{15,16}$, relacionados à malha de tensão do controle Droop, apresentam uma grande sensibilidade ao aumento no comprimento do alimentador 
G. Estes modos têm migração oposta, entretanto, devido ao menor $\zeta$ do $\lambda_{13,14}$, pode-se concluir que o afastamento elétrico das FEDs tem um impacto positivo na estabilidade dinâmica do sistema. Por outra parte, $\circ \lambda_{13,14}$ mostra-se positivamente sensível ao incremento da relação $R / X$, sendo que $\circ \lambda_{15,16}$ é praticamente insensível. Já o $\lambda_{19,20}$, associado à malha do PLL, se mostra insensíveis às variações nos parâmetros do alimentador.
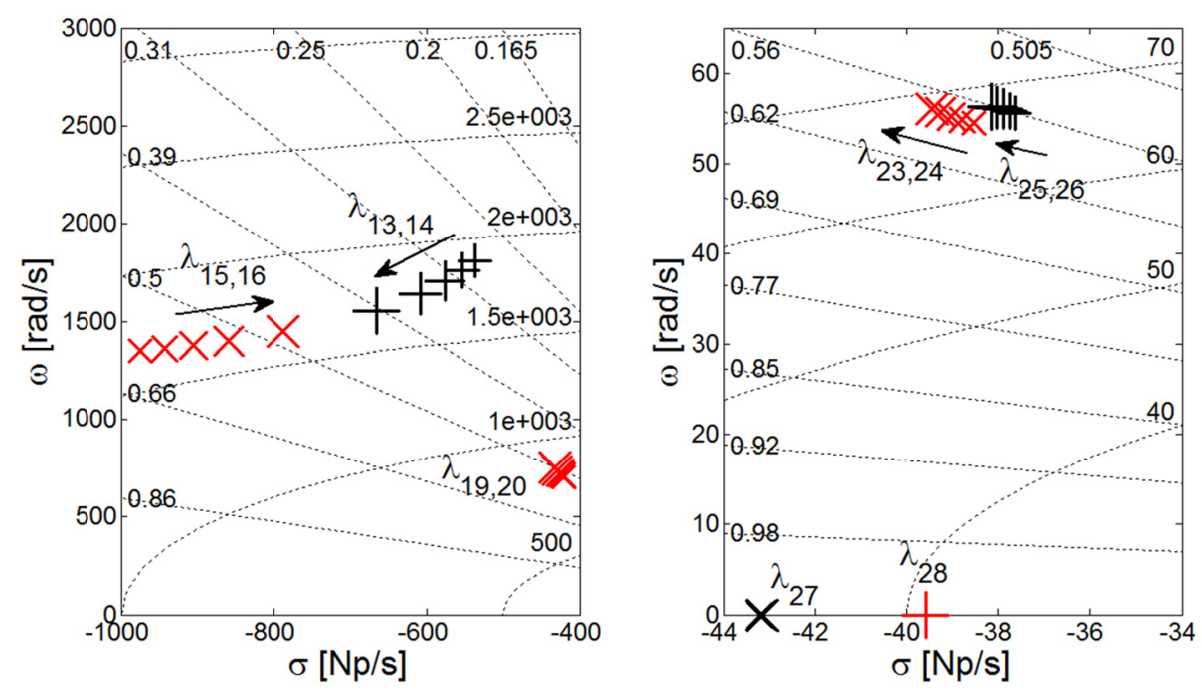

Figura 5.26 - Lugar geométrico dos modos dominantes em função do comprimento do alimentador $\boldsymbol{G}$ - Área C
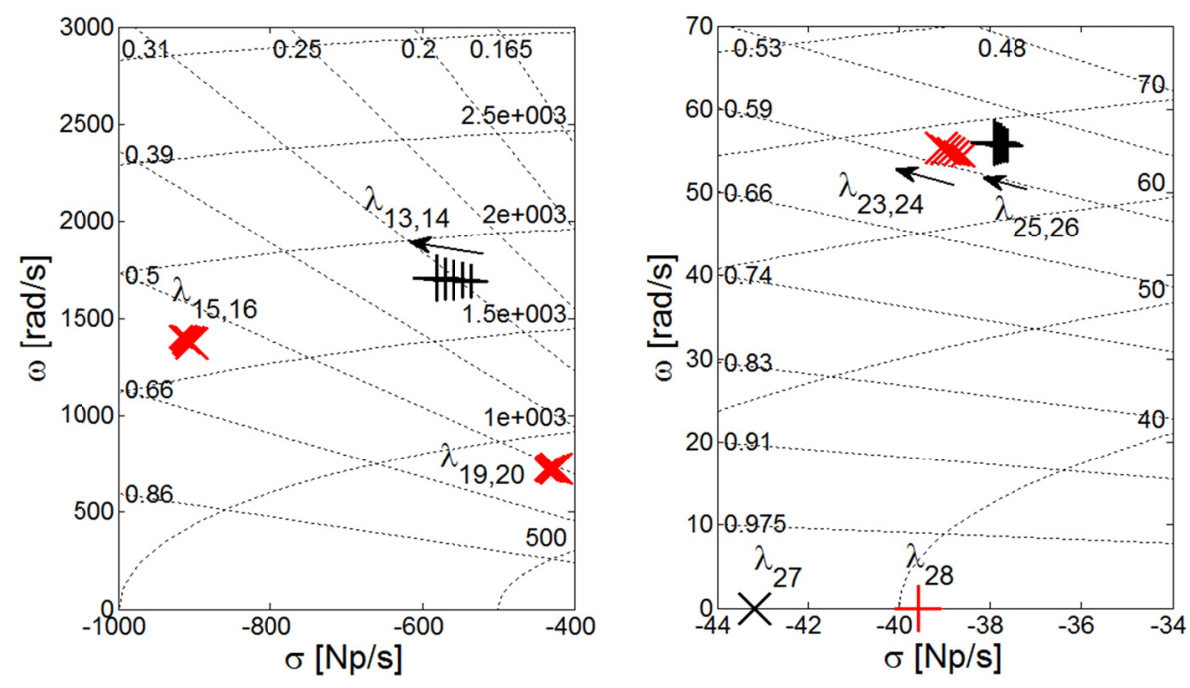

Figura 5.27 - Lugar geométrico dos modos dominantes em função da relação R/X do alimentador $\mathbf{G}$ - Área C 


\subsubsection{Interface da $F E D_{5}$ com Controle Droop+F}

Os resultados da análise modal mostraram que todos os autovalores do sistema são insensíveis a incorporação da malha de controle secundário no inversor Droop. A constante de tempo do controle secundário de frequência apresenta um valor de $0.93 \mathrm{~s}$, valor ligeiramente inferior ao obtido para operação individual do Inversor Droop+F.

\section{5 Área Total}

Após analisar a estabilidade dinâmica das áreas individualmente, agora se analisa a estabilidade da MG completa, como mostrado na Figura 5.1. Neste caso define-se a operação nominal do sistema como aquela condição que apresenta: carga ativa nominal (os dois motores de indução ligados com carga mecânica nominal) e carga passiva coincidente máxima ( $173.5 \mathrm{~kW}) \mathrm{com} \mathrm{fp}=0.92$ indutivo. Todas as FEDs, com exceção da $F E D_{1}$, são configuradas para injetar potência ativa nominal no sistema. A barra 3 é a barra de folga do sistema (slack), consequentemente a potência ativa de saída da $F E D_{1}$ compensa o desbalanço de potência do sistema. As FEDs não formadoras de rede operam com fp unitário. Já o nível de tensão a ser regulado pelas FEDs formadoras de rede no seu ponto de conexão foi calculado através de um fluxo de potência (Apêndice $B$ ), de forma a não superar os limites de reativos de cada uma das FEDs. A $F E D_{4}$ opera com controle Droop $+\mathrm{F}$ enquanto a $F E D_{5}$ opera com controle Droop.

A Tabela 5.6 mostra os principais autovalores do sistema correspondentes à operação nominal do sistema. Todos os autovalores têm parte real negativa, indicando uma condição de estabilidade para o ponto de operação considerado. A Figura 5.28 mostra os principais autovalores do sistema plotados no plano complexo.

A análise da Figura 5.28 mostra um adequado amortecimento de todos os modos, indicando uma boa margem de estabilidade dinâmica do sistema. Como esperado, o 
modo eletromecânico associado à dinâmica do motor diesel e do regulador de velocidade da $F E D_{7}\left(\lambda_{47,48}\right)$ apresenta o menor $\zeta$ (igual a 0.16 ), devido principalmente ao seu maior afastamento elétrico das outras FEDs, especialmente da $F E D_{4}$ e $F E D_{5}$ que operam com controle Droop+F e Droop. Todavia, este valor é muito superior ao registrado para operação individual do $\mathrm{GGD}(\zeta=0.1)$. Já os modos eletromecânicos associados à dinâmica do motor diesel e do regulador de velocidade da $F E D_{1}$ e $F E D_{3}\left(\lambda_{43,44}\right.$ e $\left.\lambda_{39,40}\right)$ apresentam valores muito superiores de $\zeta(0.21$ e 0.24$)$.

Tabela 5.6 - Autovalores do sistema completo - operação nominal

\begin{tabular}{cccc}
\hline Autovalor & $\sigma \pm j w$ & Autovalor & $\sigma \pm j w$ \\
\hline 1,2 & $-967.06 \pm j 1630.9$ & 29,30 & $-20.44 \pm j 78.66$ \\
3,4 & $-962.31 \pm j 1601.8$ & 31,32 & $-40.28 \pm j 58.06$ \\
5,6 & $-792.99 \pm j 1592$ & 33,34 & $-39.44 \pm j 58.14$ \\
7,8 & $-904.05 \pm j 1525.7$ & 35,36 & $-36.44 \pm j 56.99$ \\
9,10 & $-869.66 \pm j 1516.8$ & 37,38 & $-35.39 \pm j 56.35$ \\
11,12 & $-692.77 \pm j 1399.5$ & 39,40 & $-8.93 \pm j 35.74$ \\
13,14 & $-498.21 \pm j 742.05$ & 41,42 & $-19.06 \pm j 27.0$ \\
15,16 & $-430.12 \pm j 668.9$ & 43,44 & $-6.931 \pm j 31.71$ \\
17,18 & $-206.83 \pm j 339.59$ & 45,46 & $-24.97 \pm j 15.65$ \\
19,20 & $-142.20 \pm j 348.07$ & 47,48 & $-4.121 \pm j 25.280$ \\
21,22 & $-68.21 \pm j 368,71$ & 49,50 & $-11.657 \pm j 1.362$ \\
23,24 & $-76.12 \pm j 365.85$ & 51,52 & $-2.846 \pm j 6.088$ \\
25,26 & $-107.2 \pm j 334.9$ & 53,54 & $-2.204 \pm j 1.793$ \\
27,28 & $-16.08 \pm j 103.43$ & & \\
\hline
\end{tabular}

De forma similar, o modo eletromecânico $\lambda_{51,52}$, associado ao sistema de excitação da $F E D_{7}$ apresenta o menor $\zeta$ (igual a 0.42 ), valor também superior ao registrado para operação individual do $G G D(\zeta=0.3)$. Já os modos eletromecânicos associados ao sistema de excitação da $F E D_{1}$ e $F E D_{3}\left(\lambda_{53,54}\right.$ e $\left.\lambda_{49,50}\right)$ apresentam valores extremamente altos de $\zeta$ ( 0.78 e 0.99$)$.

Nota-se que os modos associados ao controle de potência do inversor com Controle Droop e Droop+F $\left(\lambda_{41,42}\right.$ e $\left.\lambda_{45,46}\right)$, da $F E D_{5}$ e $F E D_{4}$ respectivamente, são 
complexos, porém com valores elevados de $\zeta$ (0.58 e 0.85). Os modos associados ao controle de potência dos inversores com controle P-Q $\left(\lambda_{31,32}, \lambda_{33,34}, \lambda_{35,36} \mathrm{e}\right.$ $\lambda_{37,38}$ ) apresentam valores de $\zeta$ na faixa de 0.55 , valor similar ao registrado para operação individual. Também como esperado, devido à proximidade elétrica entre a $F E D_{4}$ e a $F E D_{3}$, esta ultima apresenta o maior transitório de correntes de CC nos enrolamentos do estator dentre todos os GGDs $\left(\zeta=0.37\right.$, modo $\left.\lambda_{19,20}\right)$. Já os outros modos da Tabela 5.6 não apresentam variações significativas se comparados à operação individual das FEDs.
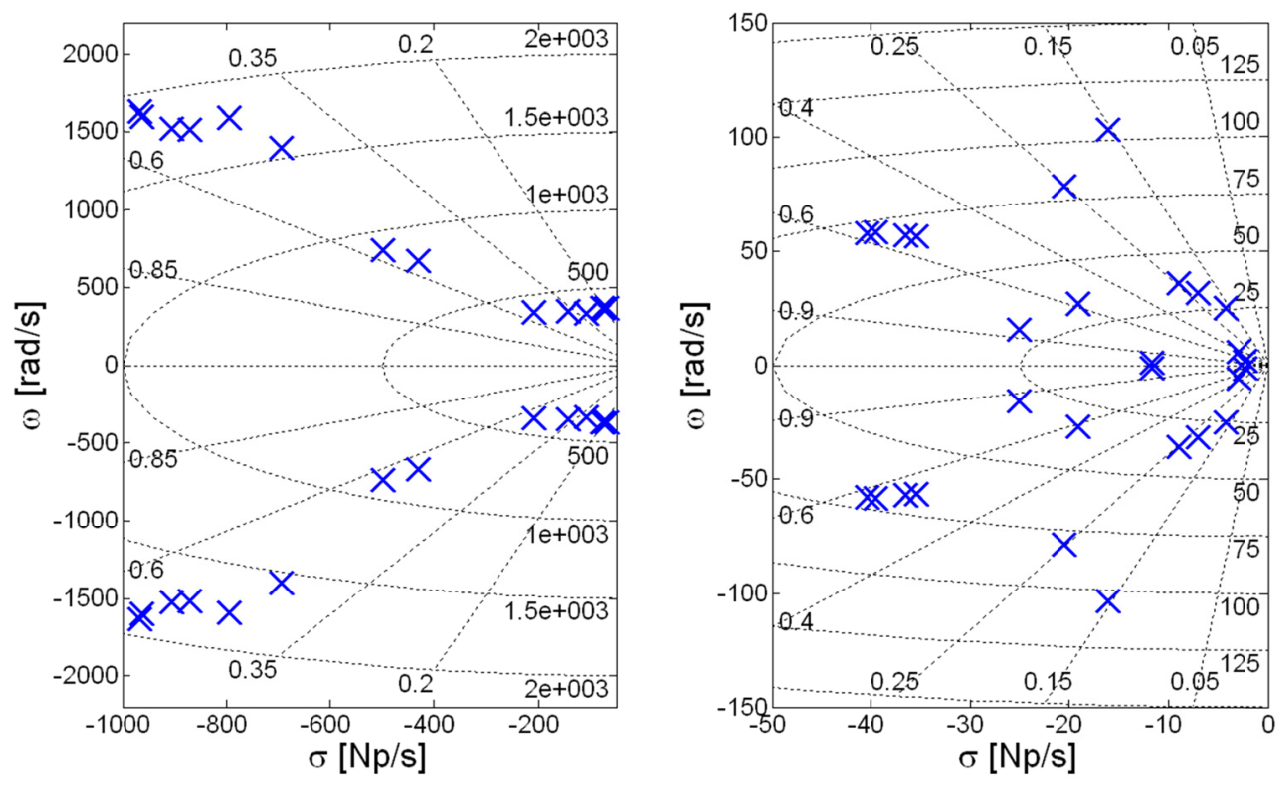

Figura 5.28 - Autovalores do sistema completo - operação nominal

A seguir se analisa a estabilidade dinâmica da MG em regime de carga leve. Para isto, simula-se uma redução de $50 \%$ nas cargas passivas do sistema. As FEDs não formadoras de rede continuam a operar em potência nominal, com fp unitário. Já as FEDs formadoras de rede operam em $50 \%$ da sua capacidade nominal. O nível de tensão a ser regulado pelas FEDs formadoras de rede no seu ponto de conexão foi calculado através de um fluxo de potência (Apêndice $B$ ), de forma a não superar os limites de reativos de cada uma das FEDs. A Tabela 5.7 mostra os principais autovalores do sistema para este regime de carga. Todos os autovalores têm parte real negativa, indicando uma condição de estabilidade para o ponto de operação considerado. 
Ao comparar a Tabela 5.6 com a Tabela 5.7, não se percebe grandes variações no $\zeta$ nem no $w$ dos autovalores em função do nível de carga da MG.

Tabela 5.7 - Autovalores do sistema completo - carga leve

\begin{tabular}{cccc}
\hline Autovalor & $\sigma \pm j w$ & Autovalor & $\sigma \pm j w$ \\
\hline 1,2 & $-978.24 \pm \mathrm{j} 1630.3$ & 29,30 & $-23.70 \pm \mathrm{j} 78.49$ \\
3,4 & $-964.50 \pm \mathrm{j} 1604.0$ & 31,32 & $-39.75 \pm \mathrm{j} 58.61$ \\
5,6 & $-784.19 \pm \mathrm{j} 1596.7$ & 33,34 & $-37.52 \pm \mathrm{j} 57.14$ \\
7,8 & $-908.04 \pm \mathrm{j} 1530.0$ & 35,36 & $-36.67 \pm \mathrm{j} 57.32$ \\
9,10 & $-872.36 \pm \mathrm{j} 1517.2$ & 37,38 & $-35.56 \pm \mathrm{j} 56.69$ \\
11,12 & $-655.38 \pm \mathrm{j} 1406.3$ & 39,40 & $-8.99 \pm \mathrm{j} 33.92$ \\
13,14 & $-512.51 \pm \mathrm{j} 737.75$ & 41,42 & $-19.11 \pm \mathrm{j} 27.0$ \\
15,16 & $-440.38 \pm \mathrm{j} 667.69$ & 43,44 & $-7.86 \pm \mathrm{j} 31.96$ \\
17,18 & $-282.99 \pm \mathrm{j} 344.11$ & 45,46 & $-24.95 \pm \mathrm{j} 15.23$ \\
19,20 & $-142.61 \pm \mathrm{j} 347.96$ & 47,48 & $-3.82 \pm \mathrm{j} 23.06$ \\
21,22 & $-68.02 \pm \mathrm{j} 368,78$ & 49,50 & $-12.19 \pm \mathrm{j} 3.33$ \\
23,24 & $-76.31 \pm \mathrm{j} 365.92$ & 51,52 & $-3.42 \pm \mathrm{j} 6.15$ \\
25,26 & $-108.2 \pm \mathrm{j} 335.23$ & 53,54 & $-1.63 \pm \mathrm{j} 1.89$ \\
27,28 & $-16.22 \pm \mathrm{j} 99.97$ & & \\
\hline
\end{tabular}

Da análise realizada na Seção 5.3, fica evidente que a $F E D_{4}$ e $F E D_{5}$ são as fontes responsáveis pela grande minimização dos transitórios eletromecânicos do sistema. Por esta razão, considera-se importante realizar uma análise de estabilidade dinâmica do sistema considerando a contingência de uma destas fontes. Escolhe-se a $\mathrm{FED}_{4}$ por ser a fonte de maior potência nominal. A Tabela 5.8 mostra as variações no $\zeta$ dos autovalores associados aos modos eletromecânicos dois GGDs, quando o sistema opera com carga coincidente máxima, sem a presença da $F E D_{4}$. As três primeiras linhas da tabela correspondem aos modos eletromecânicos associados à dinâmica do motor diesel e do regulador de velocidade. As três últimas linhas correspondem aos modos eletromecânicos associados ao sistema de excitação.

Como se esperava, a ausência da $F E D_{4}$ tem um impacto negativo em todos os modos eletromecânicos dos GGDs. Nota-se que, para o modo eletromecânico associado à dinâmica do motor diesel e do regulador de velocidade, a $F E D_{3}$ 
apresenta a maior variação negativa do $\zeta$. Já para o modo eletromecânico associado ao sistema de excitação, a maior variação do $\zeta$ apresenta a $F E D_{7}$ Estes resultados corroboram as conclusões das análises anteriores e mostram a importância das FEDs acopladas à rede através de inversores Droop e/ou Droop+F, na estabilidade dinâmica do sistema.

Tabela 5.8 - Variação do fator de amortecimento dos modos eletromecânicos Sistema em contingência

\begin{tabular}{ccccc}
\hline FED & Autovalor & $\begin{array}{c}\text { Operação } \\
\text { Normal }\end{array}$ & $\begin{array}{c}\text { Contingência } \\
\text { Perda da } F E D_{4}\end{array}$ & $\begin{array}{c}\text { Variação } \\
{[\%]}\end{array}$ \\
\hline 1 & 39,40 & 0.24 & 0.19 & -20.8 \\
3 & 43,44 & 0.21 & 0.13 & -38.0 \\
7 & 47,48 & 0.16 & 0.13 & -18.7 \\
1 & 49,50 & 0.99 & 0.77 & -22.2 \\
3 & 53,54 & 0.78 & 0.69 & -11.5 \\
7 & 51,52 & 0.42 & 0.25 & -40.4 \\
\hline
\end{tabular}

A seguir se analisa a estabilidade da MG em função da potência ativa injetada pela $F E D_{2}$ e $F E D_{6}$ no sistema. A potência ativa das duas FEDs foi variada ao mesmo tempo entre 0 e $1.0 \mathrm{pu}$, em passos de 0.25 pu. O lugar geométrico dos modos que maiores variações apresentaram se mostra na Figura 5.29.

Como discutido na Seção 5.2, a injeção de potência ativa através de um inversor com controle $\mathrm{P}-\mathrm{Q}$ praticamente não afeta os modos oscilatórios de baixa frequência do sistema. Todavia, a análise da Figura 5.29 mostra que os modos $\lambda_{5,6}$ e $\lambda_{11,12}$, associados à malha de controle dos PLLs, são negativamente sensíveis ao incremento de $P_{F E D 2}$ e $P_{F E D 6}$, com perda significativa do $\zeta$ e/ou $w$.

Uma análise similar foi realizada para variações no $Q_{F E D 2}$ e $Q_{F E D 4}$. A potência reativa das duas FEDs foi variada entre -0.75 e +0.75 pu em passos de 0.375 pu. $O$ lugar geométrico dos modos que maiores variações apresentaram se mostra na Figura 5.30. 


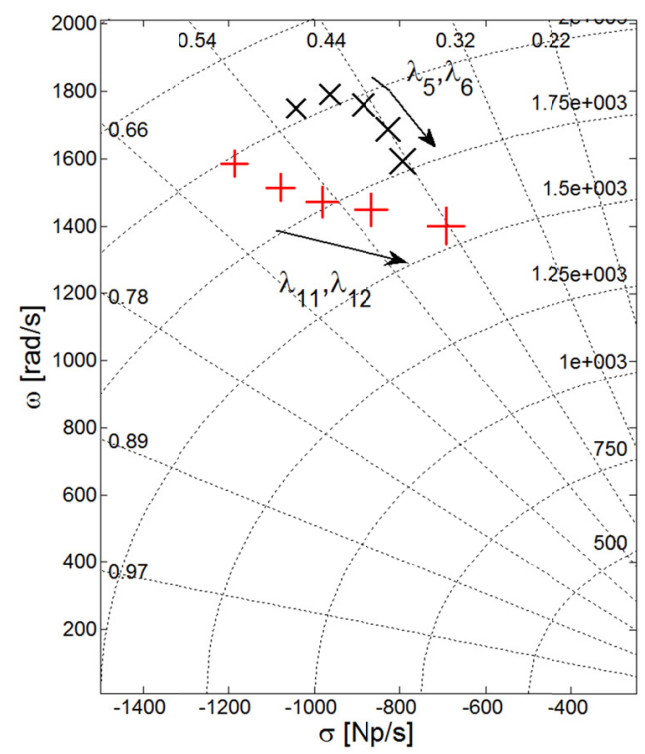

Figura 5.29 - Lugar geométrico dos modos mais sensíveis às variações no $\boldsymbol{P}_{F E D 2}$ e $\boldsymbol{P}_{\text {FED } 6}$ - Sistema completo

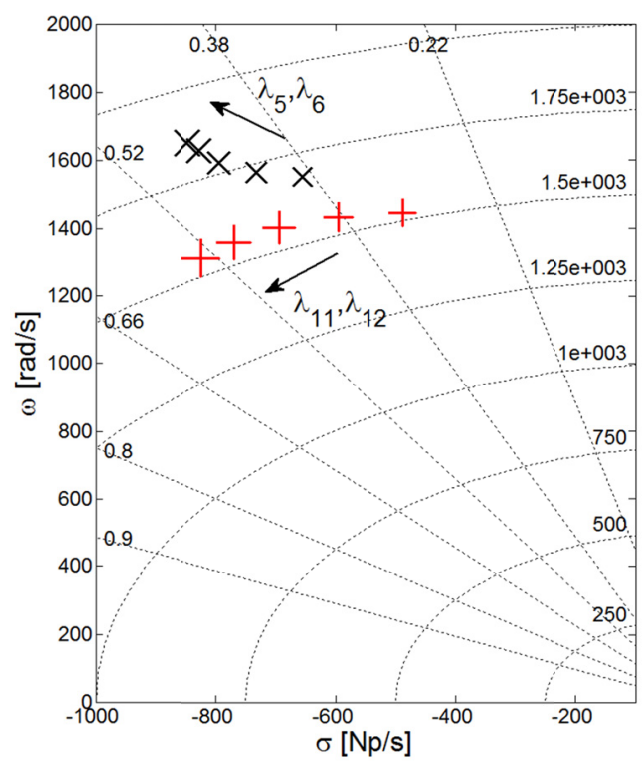

Figura 5.30 - Lugar geométrico dos modos mais sensíveis às variações no $\boldsymbol{Q}_{F E D 2}$ e $\boldsymbol{Q}_{\text {FED6 }}$ - Sistema completo

Diferentemente do observado na Seção 5.2, na presença de FEDs com controle Droop e/ou Droop+F no sistema, a injeção de reativos por parte das FEDs com Controle $\mathrm{P}-\mathrm{Q}$ não afeta a posição dos modos eletromecânicos dos GGDs. Os modos que maiores variações apresentam estão associados à malha de controle dos PLLs, mostrando neste caso uma sensibilidade positiva à injeção de reativos com aumento de $\zeta$ elou $w$. 


\section{Conclusões e sugestões para trabalhos futuros}

\subsection{Conclusões}

Neste trabalho foi analisada a estabilidade dinâmica de MGs ilhadas com controle autônomo de tensão e frequência. A análise foi realizada no espaço de estados, com metodologias baseadas nas teorias que envolvem os autovalores e autovetores da matriz de estado do sistema.

Diferentemente de outros trabalhos realizados nesta área, esta tese focou na análise da característica "ligar e usar" (do inglês plug and play) que o UCM deve proporcionar a sua respectiva FED, característica chave para o desenvolvimento modular de uma MG, e que deve garantir que uma FED comercial, com os ajustes padrões de fábrica, possa ser adicionada a uma MG sem a necessidade de recalibrar, ou até mesmo reconfigurar, as malhas de controle das FEDs existentes.

Embora existam vários tipos de FEDs convencionais e/ou alternativas que podem compor uma MG, para propósitos do estudo da estabilidade dinâmica, neste trabalho as fontes foram divididas em dois grupos: FEDs acopladas à rede através de inversores e FEDs diretamente ligadas na rede, mais especificamente, GGDs equipados com geradores síncronos convencionais com reguladores de tensão e velocidade. Dentre as várias técnicas de controle disponíveis para a operação em paralelo de inversores, três estratégias de controle mais amplamente utilizadas em aplicações de MG foram estudadas: (a) Controle P-Q, (b) Controle Droop e (c) Controle Droop $+F$.

Com o objetivo de analisar as implicações da característica "ligar e usar" do ponto de vista da estabilidade dinâmica, no Capítulo 4 se realizou o ajuste individual das FEDs modeladas no Capítulo 3. Para simular o ajuste padrão de fábrica, as FEDs (ou os inversores associadas a estas) foram calibradas de forma a atender às especificações da resposta transitória segundo Normas. Inicialmente foram dedicados esforços à calibração das malhas de controle dos GGDs. Para isto optouse por definir uma função custo capaz de avaliar quantitativamente a resposta dinâmica dos GGDs. Técnicas de otimização aplicadas a esta função permitiram 
obter os parâmetros das malhas de controle dos reguladores de tensão e frequência que melhor desempenho dinâmico apresentaram, com resposta rápida e suave dos GGDs. Já para a calibração das malhas de controle dos Inversores se utilizou uma técnica diferente. Valores de ajuste preliminares foram obtidos através do enfoque clássico de polos e zeros, e resposta em frequência do sistema; utilizando-se para isto valores típicos de projeto da teoria de controle. Entretanto, ajustes finos foram realizados através do enfoque dos autovalores da matriz de estado do sistema. Os valores adequados dos ganhos dos controladores assim obtidos (sem ajustes posteriores) foram utilizados para analisar a estabilidade dinâmica de combinações de FEDs no Capítulo 5.

Os parâmetros operacionais dos GGDs foram obtidos diretamente dos catálogos dos fabricantes. Todavia, esforços adicionais foram realizados para converter estes parâmetros operacionais em fundamentais com a maior precisão possível, sem utilizar as clássicas simplificações matemáticas encontradas na literatura especializada, simplificações que podem acarretar erros significativos na estimação dos parâmetros fundamentais de geradores de pequeno porte.

$\mathrm{Na}$ impossibilidade de poder determinar com precisão os parâmetros elétricos associados à interface dos inversores com a rede através das informações fornecidas pelo fabricante, uma metodologia para o projeto desta interface foi apresentada neste trabalho. Esta metodologia visa atender tanto aos requisitos de qualidade de energia (especificamente no referente à distorção harmônica), quanto ao desempenho dinâmico do inversor. $O$ atendimento às especificações de distorção harmônica máxima segundo Normas foi corroborado através de simulações computacionais no ambiente Matlab/Simulink®. Já o adequado desempenho dinâmico da interface ficou evidenciado na seção de testes e resultados do capítulo 4 e 5.

Nenhuma das diferentes combinações de FEDs analisadas no Capítulo 5 mostrou sinais de instabilidade dinâmica em condições nominais de operação, apresentando todas elas uma margem de estabilidade adequada para operação de sistemas de potência, sendo que em nenhum dos casos analisados viu-se a necessidade de recalibrar ou reconfigurar as malhas das FEDs previamente calibradas para operação individual no Capítulo 4. Este resultado positivo e alentador prova que, do 
ponto de vista da estabilidade dinâmica, MGs formadas por FEDs (com as características mostradas nesta tese) podem ter um crescimento modular sem a necessidade de uma engenharia especializada para recalibrar, ou até mesmo reconfigurar, as malhas de controle das FEDs existentes (característica ligar e usar).

Embora todas as combinações de FEDs analisadas no Capítulo 5 apresentaram desempenho satisfatório, importantes resultados relacionados às interações entre as FEDs, assim como a configuração da MG foram obtidos; resultados que se resumem a seguir:

a) A operação conjunta de um GGD, com um inversor com controle P-Q operado com fp unitário, reduz ligeiramente as oscilações eletromecânicas do sistema, especialmente quando o GGD opera com carga média ou leve. Todavia, a injeção de reativos por parte do inversor afeta negativamente o amortecimento destes modos. A distância elétrica entre estas FEDs não afeta as oscilações de baixa frequência do sistema, entretanto recomenda-se minimizar seu afastamento elétrico de forma a garantir um adequado amortecimento das oscilações de alta frequência associadas à malha de controle do PLL do inversor P-Q. O incremento da relação $\mathrm{R} / \mathrm{X}$ do alimentador que interconecta as duas FEDs ajuda a minimizar estas oscilações.

b) A operação conjunta de um GGD com um inversor com controle Droop ou Droop $+F$ tem um impacto profundamente positivo na estabilidade dinâmica do sistema, com aumento significativo do fator de amortecimento e frequência das oscilações eletromecânicas do sistema, independentemente da injeção e/ou absorção de reativos por parte do inversor. Todavia, a distância elétrica entre as fontes tem influência direta na estabilidade do sistema. Recomenda-se minimizar a distância entre este tipo de fontes de forma a reduzir as oscilações eletromecânicas do sistema, embora o transitório das correntes de estator (com constantes de tempo muito menores) seja menos amortecido. $O$ incremento da relação $R / X$ do alimentador que interconecta as duas FEDs ajuda a minimizar estas oscilações de alta frequência, sem maiores repercussões no amortecimento das oscilações de baixa frequência. Por outra parte, embora valores pequenos de droop de potência do inversor sejam desejáveis (aproximadamente 1\%), cuidados especiais devem ser tomados na calibração dos droops para evitar sobrecargas inadmissíveis do inversor 
durante os transitórios de aceitação de carga. Embora o controle Droop $+F$ não apresente benefícios extras sobre o controle Droop (do ponto de vista da estabilidade dinâmica), permite a rápida restauração da frequência do sistema ao seu valor programado, minimizando o período de sobrecarga do inversor durante o transitório de aceitação de carga.

c) $\mathrm{Na}$ operação conjunta de inversores com controle Droop e/ou Droop+F, recomenda-se o afastamento elétrico entre as FEDs, pois esta ação tem um impacto positivo significativo no amortecimento das oscilações de baixa e alta frequência do sistema, além dos benefícios na regulação de tensão e diminuição de perdas ativas no sistema de distribuição.

d) A operação conjunta de inversores com controle Droop (ou Controle Droop+F) e controle $\mathrm{P}-\mathrm{Q}$ apresenta a maior margem de estabilidade dinâmica dentre todas as combinações analisadas, no referente às oscilações de baixa frequência. Todavia, transitórios de alta frequência associados à malha de tensão do inversor Droop tornam-se menos amortecidos nesta configuração. $O$ afastamento elétrico entre as fontes é desejável, especialmente através de alimentadores com relação R/X alta, pois minimiza estas oscilações.

e) Quando um inversor Droop (ou Droop $+F$ ) é acrescentado a um sistema elétrico formado por um GGD e um inversor com controle $P-Q$, o impacto negativo na estabilidade dinâmica devido à injeção de reativos por parte do inversor P-Q desaparece, tornando-se o sistema insensível às variações na potência reativa injetada pelo inversor $\mathrm{P}-\mathrm{Q}$.

f) Motores de indução com carga mecânica nominal aplicada ao eixo não têm um impacto negativo pronunciado na estabilidade dinâmica do sistema. Todavia, a sobrecarga dos mesmos reduz drasticamente a margem de estabilidade dinâmica do sistema, especialmente dos modos eletromecânicos dos GGDs. A presença de inversores Droop e/ou Droop $+F$ ajuda a minimizar este impacto negativo. 


\subsection{Sugestões para trabalhos futuros}

Como trabalhos futuros sugerem-se:

- Incorporação nos estudos de estabilidade dinâmica de outros tipos de FEDs ligadas diretamente na rede, tais como grupos geradores diesel com geradores de indução e turbinas eólicas com geradores de indução de velocidade fixa.

- Análise da estabilidade dinâmica de agrupamentos de múltiplas MGs através de alimentadores de BT, trabalhando isoladamente assim como em paralelo com a rede convencional.

- Análise da estabilidade transitória das MGs assim como estudos relacionados às proteções do sistema. 


\section{Referências Bibliográficas}

1. ANEEL. Nota Técnica no. 0043/2010-SRD/ANEEL. ANEEL. [S.I.], p. 20. 2010.

2. DUNGAN, R.C.; MCGRANAGHAN, M.F.; SANTOSO, S. Electrical Power Systems Quality. 2. ed. [S.I.]: McGraw-Hill, 2004.

3. BORBELY, A.M.; KREIDER, J.F. Distributed generation: the power paradigm for the new millenium. Washington: CRC, 2001.

4. CHIRADEJA, P.; RAMAKUMAR, R. An approach to quantify the technical benefits of distributed generation. IEEE Transactions on Energy Conversion, v. 19, dez. 2004.

5. LASSETER, R.; AKHIL, A.; MARNAY, C. Integration of distributed energy resources: the CERTS Microgrid Concept. eScholarship-University of California, California, 04 jan. 2002.

6. SOUZA, A. R. Conexão de geração distribuida em redes de distribuição. 108 p. Dissertação (Mestrado) - Universidade Federal do Paraná. Paraná. 2009.

7. CHOWDHURY, S.; CROSSLEY, P. Microgrids and active distribution networks. Institution of Engineering and Technology. London: UK: IET Renewable energy series, 2009.

8. NIKNAM, T.; RANJBAR, A.M.; SHIRANI, A.R. Impact of distributed generation on Volt/Var control in distribuiton networks. In: IEEE Bologna PowerTech Conference, Bologna, p. 7, 2003.

9. MARNAY,C.; VENKATARAMANAN, G. Optimal technology selection and operation of commercial-building Microgrids. IEEE Transaction on Power Systems, v. 23, n. 3, p. 8, ago. 2008. 
10. LOPES,J.A.; MOREIRA, C.L.; MADUREIRA, A.G. Defining control strategies for Microgrids islanded operation. IEEE Transactions on Power Systems, v. 21, n. 2, maio 2006.

11. LASSETER, R.H.; PIAGI, P. Control and design of Microgrid components: final project report. University of Wisconsin. Madison, p. 257. 2006.

12. JAYAWARNA, N.; WU, X.; ZHANG, Y. Eletrical stability of large scale integration of micro generation into low voltage grids. School of Eletrical Engineering. Manchester, p. 23. 2002.

13. GRAINGER, J.; STEVENSON, W. Análisis de sistemas de potencia. México: McGraw-Hill, 1996.

14. PIAGI,P.; LASSETER, R.H. Autonomous control of Microgrids. In: IEEE PES Meeting, Montreal, p. 8, jun. 2006.

15. MANWELL, J.F.; ROGERS, A.; HAYMAN, G. HYBRID2: a hybrid system simulation model: theory manual. MASSACHUSETTS: Renewable Energy Research Laboratory, Department of Mechanical Engineering, UNIVERSITY OF MASSACHUSETTS, 2006. 293 p.

16. BORGES, C.L.; FALCÃO, D.M. Optimal distributed generation allocation for reliability, losses and voltage improvement. Eletrical Power and Energy Systems, ELSEVIER, v. 28, p. 8, abr. 2006.

17. CAIRE, R.; MORIN, R.; FONTELA, M. Voltage management of distributed generation in distribution networks. In: Power Engineering Society General Meeting, IEEE, p. 6, 2003.

18. CELLI, G.; GHIANI, E.; PILO, F. Voltage profile optimization with distributed generation. Power Tech, IEEE , p. 7, 2005.

19. AHMED, M.; ERLICH, A. Impact of distributed generation on the stability of electrical power systems. In: Power Engineering Society General Meeting IEEE, p. 8, 2005. 
20. CARDELL, J.; ILIC, M. Maintaining stability with distributed generation in a restructured industry. [Report] Carnegie Mellon Electricity Industry Center. [S.I.]. 2002.

21. ENGLER, A. Control of parallel operating battery inverters. [Report] Institut für Solare Energieversorgungstechnik (ISET) e.V., Kassel, Germany, 2000. 4.

22. BRABANDERE, K.; BOLSENS, B.; KEYBUS, J. A voltage and frequency droop control method for parallel inverters. In: Anual IEEE Power Electronics Specialists Conference, Aachen, p. 7, 2004.

23. LASSETER, R.; PIAGI, P. Providing premium power through distributed resources. In: Proceedings of the 33rd Hawaii International Conference on Systems Sciences, Hawaii, p. 9, 2000.

24. LOPES, J.A.; MOREIRA, C.L.; RESENDE, F.O. Control strategies for Microgrids black start and islanded operation. [Report] INESC Porto Instituto de Engenharia de Sistemas e Computadores do Porto. Porto, p. 21. 2005.

25. OSIKA, O. Stability of Microgrids and inverter-dominated grids with high share of decentralised sources. 112 p. Tese (Doutorado) - Universidade de Kassel. Kassel. 2005.

26. COELHO, E.A.; CORTIZO, P. C.; GARCIA, F.D. Small-signal stability for parallelconnected inverters in stand-alone AC supply systems. IEEE Transactions on Industry Applications, v. 38, n. 2, p. 10, abr. 2002.

27. POGAKU, N.; PRODANOVIC, M.; GREEN, T. Modeling, analysis and testing of autonomous operation of an invertes-based Microgrid. IEEE Transactions on Power Electronics, v. 22, n. 2, p. 13, mar. 2007.

28. MAJUMDER, R.; FELLOW, A.G.; LEDWICH, G. Stability analysis and control of multiple converter based autonomous microgrid. In: IEEE International Conference on control and Automation, Christchurch, p. 7, dez. 2009. 
29. KUNDUR, P. Power system stability and control. California: McGraw-Hill, 1994.

30. MACHOWSKI, J.; BIALEK, J.; BUMBY, J. Power systems dynamics: stability and control. 2. ed. Wiltshire: John Wiley \& Sons, 2008.

31. OGATA, K. Modern control engineering. 4. ed. [S.I.]: Prentice Hall, 2002.

32. LARA, O.; JENKINS, N.; CARTWRIGHT, J. Wind energy generation: modelling and control. Chippenham - Reino Unido: John Wiley \& Sons, Ltd, 2009.

33. KAPLAN, W. Advanced calculus. 5. ed. Ann Arbor, Michigan: Pearson AddisonWesley, 2002.

34. KRAUSE, P.; WASYNCZUK, O.; SUDHOFF, S. Electric machinery and drive systems. 2. ed. New York, USA: John Wiley \& Sons, 2002.

35. MOHAMED, Y. A. R.; SAADANY, E. F. Adaptive decentralized droop controller to preserve power sharing stability of paralleled inverters in distributed generation Microgrids. IEEE Transactions on Power Electronics, v. 23, n. 6, p. 11, nov. 2008.

36. KATIRAEI, F.; IRAVANI, M. Power management strategies for a Microgrid with multiple distributed generation units. IEEE Transactions on Power Systems, v. 21, p. 11, November 2006.

37. HTAY, M.; WIN, K. Regulator for diesel engine type stand-alone synchronous generator. World Academy of Science, Engineering and Technology, v. 42, p. 7, 2008.

38. NACFAIRE, H. (Ed.). Wind-diesel and wind autonomous energy systems. New York: ELSEVIER , 2005.

39. DIESEL GENERATOR SET SPECS. Site da CATERPILLAR. Disponivel em: <http://www.cat.com/cda/files/2268891/7/LEHX0702-01.pdf>. Acesso em: mar. 2012. 
40. STAMFORD POWER GENERATION. Sx460 Automatic voltage regulator (AVR), 2003. Disponivel em: <http://genpowerusa.com/pdf/avrs/voltageregulator-stamford-SX460-avr.pdf>. Acesso em: mar. 2012.

41. IEEE. Std 421.5. Recomended practice for excitation system models for power system stability studies. IEEE Power Engineering Society. New York. 2005.

42. HUNTER, R.; ELLIOT, G. Wind-Diesel Systems: A guide to the technology and its implementation. New York: Cambridge University Press - Prepared under the auspices of the International Energy Agency, 2005.

43. HARUNI, A. M.O.; GARGOOM, A.; HAQUE, M.E.; NEGNEVITSKY, M. Dynamic operation and control of a hybrid wind-diesel stand alone power systems. In: Applied Power Electronics Conference and Exposition, Tasmania, Australia, p. 8, 2010.

44. MUFTI, M,D; BALASUBRAMANIAN, R; TRIPATHY, S,C. Dynamic performance assessment of an isolated wind-diesel power system with superconducting magnetic energy storage unit under turbulent wind and load disturbances. International Journal of Energy Research , v. 26, p. 185-201, jan. 2002.

45. GARCIA, S, L.; IRAVANI, M, R. Hybrid wind-diesel power plantas: modeling and analysis. Saarbrücken, Alemanha: VDM Verlag Dr. Müller, 2008.

46. FURTADO, E. C. Estudo do sincronismo de múltiplos osciladores acoplados ao paralelismo de UPS. 208 p. Tese (Doutorado) - Universidade Federal de Minas Gerais. Belo Horizonte. 2009.

47. MOREIRA, C. C. L. Identification and development of Mirogrids: emergency control procedures. 279 p. Tese (Doutorado) - Universidade de Porto. Porto. 2008.

48. KROUTIKOVA, C.A.; ARAMBURO, H.; GREEN, T.C. State-space model of gridconnected inverters under current control model. IET Eletrical Power Application, London, p. 10, 2007. 
49. AKAGI, H.; WATANABE, E. Instantaneous power theory and applications to power conditioning. New Jersey: John Wiley\&Sons, 2007.

50. ENGLER, A. Applicability of droops in low voltage grids. DER JOURNAL, n. 1, p. 5, jan. 2005.

51. FRIEDEL, V. Modeling and simulation of a hybrid wind-diesel Microgrid. 109 p. Tese (Doutorado) - Electrical Engineering Royal Institute of Technology Stockholm. Stockholm, Sweden. 2009.

52. ISO. 8528-5:2005. Reciprocating internal combustion engine driven alternating current generating sets: Part 5. Genebra - Suiça, p. 34.

53. CATERPILLAR. Electric power applications, engine\&generator sizing: application and installation guide. USA: CATERPILLAR, 2008. 120 p.

54. R2008A, M. Toolbox Matlab SimPowerSystems. [S.I.]. 2008.

55. LISERRE, M.;BLAABJERG, F.; HANSES, S. Design and control of an LCL-filter based three-phase active rectifier. IEEE Transaction on Industry Applications, v. 41, p. 1281-1291, out. 2005.

56. IEEE. Std 929-200. Recommended practice for utility interface of photovoltaic (PV) systems. IEEE Standards Coordinating Committee 21 on Fuel Cells, Photovoltaics, Dispersed Generation, and Energy Storage. New York: USA, p. 32. 2000.

57. IEC. 61727:2004. Photovoltaic (PV) systems: characteristics of the utility interface. International Standard. [S.I.], p. 19. 2004.

58. IEC. 62040-3 ed.2.0. Uninterruptible power systems (UPS) - Part 3:Method of specifying the performance and test requirements. International Standard. [S.I.], p. 99. 2010. 
59. IEEE. Std 1547:2003. Standard for interconnecting distributed resources with electric power systems. IEEE Standards Coordinating Committee 21 on Fuel Cells, Photovoltaics, Dispersed Generation, and Energy Storage. [S.I.], p. 27. 2003.

60. UL. 1741:2001. Inverters, converters, and controllers for use in independent power systems. Underwriters Laboratories Inc. [S.I.], p. 174. 2001.

61. SMA. Off-grid inverter Sunny Island - Technical Description. SMA. USA, p. 228. 2011.

62. SMA. Solar inverter Sunny Boy: installation guide. SMA. USA, p. 115. 2011. 


\section{APÊNDICE A}

Neste apêndice se mostram os parâmetros elétricos dos alimentadores de distribuição internos da MG assim como a distribuição das cargas elétricas nas barras do sistema

Tabela A-1 - Parâmetros Elétricos da Rede

\begin{tabular}{cccccc}
\hline Linha & $\begin{array}{c}\text { Barra de } \\
\text { saída }\end{array}$ & $\begin{array}{c}\text { Barra de } \\
\text { chegada }\end{array}$ & $\begin{array}{c}\text { Condutor } \\
{[\text { AWG }]}\end{array}$ & $\begin{array}{c}\text { Comprimento } \\
{[\mathrm{m}]}\end{array}$ & $\begin{array}{c}\text { Impedância } \\
{[\Omega]}\end{array}$ \\
\hline A & 1 & 2 & $3 / 0$ & 50 & $0.0205+\mathrm{j} 0.020$ \\
B & 2 & 3 & $1 / 0$ & 50 & $0.0310+\mathrm{j} 0.021$ \\
C & 3 & 4 & 2 & 100 & $0.0980+\mathrm{j} 0.043$ \\
D & 1 & 5 & $2 / 0$ & 80 & $0.0400+\mathrm{j} 0.033$ \\
E & 5 & 6 & 2 & 100 & $0.0980+\mathrm{j} 0.043$ \\
F & 2 & 7 & $2 / 0$ & 150 & $0.0750+\mathrm{j} 0.062$ \\
G & 7 & 8 & 2 & 100 & $0.0980+\mathrm{j} 0.043$ \\
H & 1 & 9 & $3 / 0$ & 200 & $0.0820+\mathrm{j} 0.080$ \\
\hline
\end{tabular}

Tabela A-2 - Carga máxima coincidente

\begin{tabular}{ccc}
\hline Barra & $\begin{array}{c}\text { Carga passiva } \\
{[\mathrm{kVA}]}\end{array}$ & $\begin{array}{c}\text { Carga ativa } \\
{[\mathrm{KW}]}\end{array}$ \\
\hline 1 & 0 & 0 \\
2 & 0 & 0 \\
3 & $20.0+\mathrm{j} 8.52$ & 0 \\
4 & $10.0+\mathrm{j} 4.26$ & 0 \\
5 & $36.0+\mathrm{j} 15.33$ & 15.0 \\
6 & $7.5+\mathrm{j} 3.20$ & 0 \\
7 & $15.0+\mathrm{j} 6.39$ & 0 \\
8 & $5.0+\mathrm{j} 2.13$ & 0 \\
9 & $80.0+\mathrm{j} 34.0$ & 30.0 \\
\hline
\end{tabular}




\section{APÊNDICE B}

Neste apêndice se mostram os resultados do fluxo de potência da MG para carga nominal e leve.

\section{Fluxo de potência da MG - Carga Nominal}

\begin{tabular}{|c|c|c|c|c|c|c|c|c|c|c|}
\hline \multicolumn{8}{|c|}{ Informação das barras } & \multirow{2}{*}{\multicolumn{3}{|c|}{ Fluxo nos alimentadores }} \\
\hline \multirow[b]{2}{*}{$\begin{array}{l}\text { No. } \\
\text { Barra }\end{array}$} & \multirow[b]{2}{*}{$\begin{array}{c}\text { Tipo de } \\
\text { Barra }\end{array}$} & \multirow[b]{2}{*}{$\begin{array}{c}\text { Tensão } \\
\text { [pu] }\end{array}$} & \multirow[b]{2}{*}{$\begin{array}{l}\text { Ângulo } \\
\text { [graus] }\end{array}$} & \multicolumn{2}{|c|}{ Geração } & \multicolumn{2}{|c|}{ Carga } & & & \\
\hline & & & & [kW] & [kVAr] & {$[\mathrm{kW}]$} & [kVAr] & $\begin{array}{c}\text { À Barra } \\
\text { No. }\end{array}$ & {$[\mathrm{kW}]$} & [kVAr] \\
\hline & & & & & & & & 2 & -7.32 & -9.18 \\
\hline \multirow[t]{2}{*}{1} & --- & 0.99 & 0.37 & 0.00 & 0.00 & 0.00 & 0.00 & 5 & -13.64 & 19.74 \\
\hline & & & & & & & & 9 & 20.96 & -10.57 \\
\hline \multirow[t]{2}{*}{2} & --- & 0.99 & 0.32 & 0.00 & 0.00 & 0.00 & 0.00 & 3 & -2.48 & -10.42 \\
\hline & --- & & & & & & & 7 & -4.90 & 1.02 \\
\hline 3 & Slack & 1.00 & 0.00 & 12.84 & 23.75 & 20.00 & 8.52 & 4 & -9.77 & 4.36 \\
\hline 4 & $P Q$ & 1.02 & 0.99 & 20.00 & 0.00 & 10.00 & 4.26 & --- & --- & --- \\
\hline 5 & PV & 0.98 & 1.93 & 45.00 & 34.00 & 51.00 & 26.63 & 6 & -20.10 & 27.25 \\
\hline 6 & PV & 1.00 & 6.17 & 30.00 & 23.00 & 7.50 & 3.20 & --- & --- & --- \\
\hline 7 & PV & 1.00 & 0.79 & 15.00 & 7.56 & 15.00 & 6.39 & 8 & -4.94 & 2.16 \\
\hline 8 & $P Q$ & 1.01 & 1.28 & 10.00 & 0.00 & 5.00 & 2.13 & --- & --- & --- \\
\hline 9 & $\mathrm{PV}$ & 0.97 & -2.75 & 90.00 & 68.00 & 110.00 & 56.50 & --- & --- & --- \\
\hline
\end{tabular}


Fluxo de potência da MG - Carga Leve

\begin{tabular}{|c|c|c|c|c|c|c|c|c|c|c|}
\hline \multicolumn{8}{|c|}{ Informação das barras } & \multirow{2}{*}{\multicolumn{3}{|c|}{ Fluxo nos alimentadores }} \\
\hline \multirow[b]{2}{*}{$\begin{array}{l}\text { No. } \\
\text { Barra }\end{array}$} & \multirow[b]{2}{*}{$\begin{array}{c}\text { Tipo de } \\
\text { Barra }\end{array}$} & \multirow[b]{2}{*}{$\begin{array}{c}\text { Tensão } \\
\text { [pu] }\end{array}$} & \multirow[b]{2}{*}{$\begin{array}{l}\text { Ângulo } \\
\text { [graus] }\end{array}$} & \multicolumn{2}{|c|}{ Geração } & \multicolumn{2}{|c|}{ Carga } & & & \\
\hline & & & & {$[\mathrm{kW}]$} & [kVAr] & {$[\mathrm{kW}]$} & [kVAr] & $\begin{array}{c}\text { À Barra } \\
\text { No. }\end{array}$ & {$[\mathrm{kW}]$} & {$[\mathrm{kVAr}]$} \\
\hline & & & & & & & & 2 & -27.84 & 27.07 \\
\hline \multirow[t]{2}{*}{1} & --- & 0.99 & -2.64 & 0.00 & 0.00 & 0.00 & 0.00 & 5 & 0.00 & 1.99 \\
\hline & & & & & & & & 9 & 27.77 & -29.06 \\
\hline \multirow[t]{2}{*}{2} & --- & 1.00 & -1.31 & 0.00 & 0.00 & 0.00 & 0.00 & 3 & -21.23 & 21.15 \\
\hline & --- & & & & & & & 7 & -7.26 & 5.29 \\
\hline 3 & Slack & 1.00 & 0.00 & 17.25 & -14.17 & 10.00 & 4.26 & 4 & -14.56 & 2.32 \\
\hline 4 & $P Q$ & 1.03 & 0.98 & 20.00 & 0.00 & 5.00 & 2.13 & --- & --- & --- \\
\hline 5 & PV & 0.99 & -2.55 & 22.50 & 34.00 & 33.00 & 18.95 & 6 & -10.43 & 17.04 \\
\hline 6 & PV & 1.00 & -0.02 & 15.00 & -15.08 & 3.75 & 1.60 & --- & --- & --- \\
\hline 7 & PV & 1.00 & -0.31 & 7.50 & -0.88 & 7.50 & 3.19 & 8 & -7.39 & 1.11 \\
\hline 8 & $P Q$ & 1.01 & 0.19 & 10.00 & 0.00 & 2.50 & 1.07 & --- & --- & --- \\
\hline 9 & PV & 1.00 & -8.13 & 45.00 & 71.26 & 70.00 & 39.50 & --- & --- & --- \\
\hline
\end{tabular}

